Wesley Góis

\title{
MÉTODO DOS ELEMENTOS FINITOS GENERALIZADOS EM FORMULAÇÃO VARIACIONAL MISTA
}

Dissertação apresentada à Escola de Engenharia de São Carlos, Universidade de São Paulo, como parte dos requisitos para a obtenção do título de Mestre em Engenharia de Estruturas.

Orientador: Prof. Dr. Sérgio Persival Baroncini Proença

São Carlos 
Dedico este trabalho aos meus pais Bomfim e Creuza 


\section{AGRADECIMENTOS}

Ao Prof. Dr. Sérgio Persival Baroncini Proença, pela orientação competente, pelo comprometimento e paciência demonstrados ao longo de todo o trabalho.

Aos professores da Universidade Federal de Sergipe, em especial, Ângela Tereza Costa Sales, Antônio Santos Silva, Jorge Lima Costa, Josafá de Oliveira Filho, José Daltro Filho e Lília Cunha Góis, pelos conselhos e incentivos constantes.

Ao professor Josafá de Oliveira Filho, pela acolhida no Departamento de Engenharia de Estruturas da Escola de Engenharia de São Carlos.

Aos funcionários e professores do Departamento de Engenharia de Estruturas da Escola de Engenharia de São Carlos, pela disposição em ajudar.

Aos muitos amigos da USP/São Carlos, pelo companheirismo. Em especial a Denise Conceição, Fabiana Munhoz, Francisco Adriano, Ivan Torres, Larissa Kirchhof, Luciana Pizzo, Oscar Begambre, Rodrigo Paccola, Walter Luiz, os grandes amigos do SET, SHS, SEM e da atlética CAASO.

Aos amigos do Banco do Nordeste do Brasil S.A., pelo incentivo fundamental para que pudesse enfrentar este novo desafio.

À CAPES, pela bolsa de estudo concedida.

À minha irmã Maria Cleide e ao meu cunhado Antônio Mendonça, pela atenção e apoio.

Aos meus pais, irmãos e sobrinhos, pelo amor e carinho que sempre me deram, a minha eterna gratidão.

A Deus, ao Sagrado Coração de Jesus e à Bem-Aventurada Virgem Maria do Monte Carmelo, pela minha vida repleta de graças e fontes inesgotáveis de amor e fé. 


\section{RESUMO}

GÓIS, W. (2004). Método dos elementos finitos generalizados em formulação variacional mista. Dissertação (Mestrado) - Escola de Engenharia de São Carlos, Universidade de São Paulo, São Carlos.

Este trabalho trata da combinação entre a formulação Híbrida-Mista de Tensão (FHMT) (Freitas et al. (1996)), para a elasticidade plana, com o Método dos Elementos Finitos Generalizados (MEFG), Duarte et al. (2000). O MEFG se caracteriza como uma forma não-convencional do Método dos Elementos Finitos (MEF) que resulta da incorporação a este de conceitos e técnicas dos métodos sem malha, como o enriquecimento nodal proposto do Método das Nuvens "hp". Como na FHMT são aproximados dois campos no domínio (tensão e deslocamento) e um no contorno (deslocamento), diferentes possibilidades de enriquecimento nodal são exploradas. Para a discretização do modelo Híbrido-Misto empregam-se elementos finitos quadrilaterais com funções de forma bilineares para o domínio e elementos lineares para o contorno. Essas funções são enriquecidas por funções polinomiais, trigonométricas, polinômios que proporcionam distribuição de tensões auto-equilibradas ou mesmo funções especiais relacionadas às soluções dos problemas de fratura. Uma extensão do teste numérico abordado em Zienkiewicz et al. (1986) é proposta como investigação inicial das condições necessárias para garantia de estabilidade da resposta numérica. $\mathrm{O}$ estudo da estabilidade é completado com a análise da condição de Babuška-Brezzi (inf-sup). Esta condição é aplicada nos elementos finitos quadrilaterais híbridos-mistos enriquecidos por meio de um teste numérico, denominado de inf-sup teste, desenvolvido com base no trabalho de Chapelle e Bathe (1993). Exemplos numéricos revelam que a FHMT é uma interessante alternativa para obtenção de boas estimativas para os campos de tensões e deslocamentos, usando-se enriquecimento sobre alguns nós de malhas pouco refinadas.

Palavras-chave: Método dos Elementos Finitos; Método dos Elementos Finitos Generalizados; Formulação Variacional Mista; Estabilidade do Método dos Elementos Finitos. 


\begin{abstract}
GÓIS, W. (2004). Generelized finite element method in mixed variational formulation. MS.c. Dissertation - São Carlos School of Engineering, University of São Paulo, São Carlos.
\end{abstract}

This work presents a combination of Hybrid-Mixed Stress Model formulation (HMSMF) (Freitas et al. (1996)), to treat plane elasticity problems, with Generalized Finite Element Method (GFEM), (Duarte et al. (2000)). GFEM is characterized as a nonconventional formulation of the Finite Element Method (FEM). GFEM is the result of the incorporation of concepts and techniques from meshless methods. One example of these techniques is the nodal enrichment that was formulated in the "hp" Clouds Method. Since two fields in domain (stress and displacement) and one in boundary (displacement) are approximated in the HMSMF, different possibilities of nodal enrichment are tested. For the discretization of the Hybrid-Mixed Model quadrilateral finite elements with bilinear shape functions for the domain and linear elements for the boundary were employed. These functions are enriched with polynomial functions, trigonometric functions, polynomials that generate self-equilibrated stress distribution, or, even special functions connected with solutions of fracture problems. An extension of the numerical test cited in Zienkiewicz et al. (1986) is proposed as initial investigation of necessary conditions to assure the stability of the numerical answer. The stability study is completed with the analysis of the Babuška-Brezzi (inf-sup) condition. This last condition is applied to hybrid-mixed enrichment quadrilaterals finite elements by means of a numerical test, denominated inf-sup test, which was developed based on paper of Chapelle and Bathe (1993). Numerical examples reveal that HMSMF is an interesting alternative to obtain good estimates of the stress and displacement fields, using enrichment over some nodes of poor meshes.

Keywords: Finite Element Method; Generalized Finite Element Method; Mixed Variational Formulation; Stability of Finite Element Method. 


\section{Lista de Figuras}

Figura 2.1 - Corpo elástico submetido a forças de volume e de superfície..........

Figura 4.1 - Elemento finito quadrilateral com sistema de referência no baricentro

Figura 5.1 - Nuvens de influência para as malhas de cobertura: domínio (bidimensional) e contorno (unidimensional).

Figura 5.2 - Fissura em um campo tensional.

Figura 5.3 - Elemento utilizado para desenvolvimento das matrizes das eq.(4.11), eq.(4.12) e eq.(4.13)

Figura 7.1 - Chapa tracionada simetricamente. 64

Figura 7.2 - Chapa tracionada com fenda. 65

Figura 7.3 - Dupla simetria da chapa tracionada com fenda.

Figura 7.4 - Exemplo modelo do problema 1

Figura 7.5 - Exemplo modelo do problema 1 - representação do campo de tensões

Figura 7.6 - Discretizações adotas para análise do problema 1

Figura 7.7 - Nós da malha regular 8x9 escolhidos no enriquecimento seletivo..

Figura 7.8 - Representação do campo de tensões sem enriquecimento

Figura 7.9 - Representação do campo de tensões com enriquecimento do tipo $\left(y-y_{n \dot{o}}\right)^{2} \quad$ sobre $\sigma \quad$ e $u$ no $\quad \Omega$ - oito nós $(35,36,44,45,53,54,62$ e 63$)$

Figura 7.10 - Representação do campo de tensões com enriquecimento do tipo $\boldsymbol{d}_{\boldsymbol{e}}=1$ sobre $\sigma$ em todos os nós

Figura 7.11 - Representação do campo de tensões com enriquecimento do tipo $\boldsymbol{d}_{\boldsymbol{e}}=1$ sobre $\sigma$ - oito nós $(35,36,44,45,53,54,62$ e 63$)$

Figura 7.12 - Representação do campo de tensões com enriquecimento do tipo $\boldsymbol{d}_{e}=2$ sobre $\sigma$ - oito nós $(35,36,44,45,53,54,62$ e 63$) \ldots \ldots \ldots . .$.

Figura 7.13 - Nós enriquecidos da malha irregular $12 \times 15$.

Figura 7.14 - Representação do campo de tensões sem enriquecimento 
Figura 7.15 - Representação do campo de tensões com enriquecimento do $\operatorname{tipo}\left(y-y_{n \dot{ }}\right)^{2}+\left(x-x_{n \dot{\delta}}\right)^{2}+\left(y-y_{n \dot{\delta}}\right)\left(x-x_{n \dot{b}}\right)$ sobre $\sigma$ no $\Omega$ dezesseis nós $(64,65,77,78,90,91,103,104,116,117,129$, $130,142,143,155$ e 156$)$.

Figura 7.16 - Representação do campo de tensões com enriquecimento do $\operatorname{tipo}\left(y-y_{n \dot{\delta}}\right)^{2}+\left(x-x_{n \dot{\delta}}\right)^{2}+\left(y-y_{n \dot{\delta}}\right)\left(x-x_{n \dot{b}}\right)$ sobre $\sigma$ e $u$ no $\Omega$ - dezesseis nós $(64,65,77,78,90,91,103,104,116,117,129$, $130,142,143,155$ e 156$)$ e em $u$ no $\Gamma$ - seis nós $(78,91,104$, $117,130$ e 143$)$

Figura 7.17 - Representação do campo de tensões com enriquecimento do tipo $\boldsymbol{d}_{\boldsymbol{e}}=2$ - dezesseis nós sobre $\sigma(64,65,77,78,90,91,103,104$, $116,117,129,130,142,143,155$ e 156$)$.

Figura 7.18 - Representação do campo de tensões com enriquecimento do tipo sen $\left\lfloor\left(y-y_{n \dot{b}}\right)^{2}\right\rfloor$ sobre $\sigma$ e $u$ no $\Omega$ - todos os nós.

Figura 7.19 - Representação do campo de tensões com enriquecimento do tipo $\operatorname{sen}\left\lfloor\left(y-y_{n \dot{b}}\right)^{2}\right\rfloor$ sobre $\sigma$ no $\Omega$ - todos os nós.

Figura 7.20 - Representação do campo de tensões com enriquecimento do tipo $\cos \left\lfloor\left(\boldsymbol{x}-\boldsymbol{x}_{\boldsymbol{n} \boldsymbol{\delta}}\right)^{2}\right\rfloor$ sobre $\sigma$ e $u$ no $\Omega$ - dezesseis nós $(64,65$, $77,78,90,91,103,104,116,117,129,130,142,143,155$ e $156)$

Figura 7.21 - Exemplo modelo 2

Figura 7.22 - Exemplo modelo 2 - representação do campo de tensões próximo à ponta da fenda.

Figura 7.23 - Discretizações adotadas para análise do problema 2.

Figura 7.24 - Nós da malha regular 3x3 escolhidos no enriquecimento seletivo..

Figura 7.25 - Representação do campo de tensão Sigma - x sem e com enriquecimento em seis nós $\sigma$ no $\Omega$

Figura 7.26 - Representação do campo de tensões com enriquecimento por meio de funções que se assemelham à solução singular da Mecânica da Fratura..

Figura 7.27 - Nós da malha irregular 12x9 selecionados para 0 enriquecimento

Figura 7.28 - Representação do campo de tensões sem enriquecimento. 
Figura 7.29 - Representação do campo de tensões com enriquecimento do $\operatorname{tipo}\left(y-y_{n \dot{\delta}}\right)^{2}+\left(x-x_{n \dot{\delta}}\right)^{2}+\left(y-y_{n \dot{\delta}}\right)\left(x-x_{n \dot{\delta}}\right)$ sobre $\sigma$ - todos os nós do $\Omega$

Figura 7.30 - Representação do campo de tensões com enriquecimento do $\operatorname{tipo}\left(y-y_{n \dot{ }}\right)^{2}+\left(x-x_{n \dot{b}}\right)^{2}+\left(y-y_{n \dot{\delta}}\right)\left(x-x_{n \dot{b}}\right)$ sobre $\sigma \quad$ e $u$ todos os nós do $\Omega$

Figura 7.31 - Representação do campo de tensões com enriquecimento por meio de funções que se assemelham à solução singular da Mecânica da Fratura sobre $\sigma$ - quatro nós (40, 41, 53 e 54)

Figura 7.32 - Representação do campo de tensões com enriquecimento por meio de funções que se assemelham à solução singular da Mecânica da Fratura sobre $\sigma$ e sobre $u$ - seis nós $(26,39,40,41$, 53 e 54).

Figura 7.33 - Representação do campo de tensões com enriquecimento do tipo $\boldsymbol{d}_{e}=1$ sobre $\sigma-$ todos os nós para.

Figura 7.34 - Representação do campo de tensões com enriquecimento do tipo $\boldsymbol{d}_{\boldsymbol{e}}=2$ sobre $\sigma$ - quatorze nós para $(12,13,25,26,27,28,38$, $39,40,41,53,54,66$ e 67$)$.

Figura 7.35 - Representação do campo de tensões com enriquecimento do tipo $\operatorname{sen}\left\lfloor\left(y-y_{n \dot{\sigma}}\right)^{2}\right]$ sobre $\sigma$ no $\Omega$ - Quatorze nós. $(12,13,25$, $26,27,28,38,39,40,41,53,54,66$ e 67$)$.

Figura 7.36 - Representação do campo de tensões com enriquecimento do tipo sen $\left\lfloor\left(y-y_{n \dot{b}}\right)^{2}\right\rfloor$ sobre $\sigma$ e $u$ no $\Omega$ - todos os nós.

Figura 8.1 - Elementos quadrilaterais de quatro nós analisados pelo inf-sup teste.....

Figura 8.2 - inf-sup teste aplicado ao elemento quadrilateral de quatro nós sem enriquecimento.

Figura 8.3 - inf-sup teste aplicado ao elemento quadrilateral de quatro nós com enriquecimento sobre o campo de tensões em todos os nós com a função $(y)^{2}$

Figura 8.4 - inf-sup teste aplicado ao elemento quadrilateral de quatro nós com enriquecimento sobre o campo de tensões e deslocamentos no domínio em todos os nós com a função $(y)^{2}$

Figura 8.5 - inf-sup teste aplicado ao elemento quadrilateral de quatro nós com enriquecimento sobre o campo de tensões no domínio em todos os nós com as funções $\operatorname{sen}\left(y^{2}\right)$ e $\cos \left(x^{2}\right)$.

Figura 8.6 - inf-sup teste aplicado ao elemento quadrilateral de quatro nós com enriquecimento sobre o campo de tensões em dois os nós com a função $(y)^{2}$ 
Figura 8.7 - inf-sup teste aplicado ao elemento quadrilateral de quatro nós com enriquecimento sobre o campo de tensões em um nó com a função $(y)^{2}$.....

Figura 8.8 - inf-sup teste aplicado ao elemento quadrilateral de quatro nós com enriquecimento sobre o campo de tensões no domínio em um os nó com as funções $\operatorname{sen}\left(y^{2}\right)$ e $\cos \left(x^{2}\right)$. 


\section{Lista de Símbolos}

$\Omega$ - $\quad$ Domínio de um corpo elástico

$\Gamma$ - $\quad$ Contorno regular que limita o domínio de um corpo elástico

$\boldsymbol{b}$ - $\quad$ Vetor de forças volúmicas

$\overline{\boldsymbol{t}}$ - $\quad$ Vetor de forças de superfície

$\boldsymbol{t}$ - $\quad$ Vetor de forças de superfície (reação)

$\Gamma_{t}$ - $\quad$ Parte do contorno regular onde são impostas as forças de superfície

$\Gamma_{u}-\quad$ Parte do contorno regular onde são impostos os deslocamentos

$i, j, k$ - Índices da notação de Einstein $i, j, k, 1=1,2,3$

$x$ e $y$ - Coordenadas do sistema cartesiano

$\sigma_{i j}-\quad$ Tensor de tensões de segunda ordem

$\varepsilon_{i j}$ - Tensor das deformações de segunda ordem

$\sigma_{i j}^{0}$ - Tensor de tensões iniciais de segunda ordem

$\varepsilon_{i j}^{0}$ - Tensor de deformações iniciais de segunda ordem

$\boldsymbol{D}_{i j k \boldsymbol{l}}$ - Tensor constitutivo de rigidez de quarta ordem

$f_{i j k l}$ - Tensor constitutivo de flexibilidade

$\sigma_{j i, j}$ - Derivadas parciais do tensor de tensões de segunda ordem

$\boldsymbol{u}_{i, j}$ - Derivadas parciais do vetor deslocamento

$\boldsymbol{b}_{\boldsymbol{i}}$ - Vetor de forças volúmicas

$\boldsymbol{u}_{\boldsymbol{i}}$ - Vetor de deslocamentos

$\sigma$ - Vetor coluna formado com as componentes do tensor de tensões de segunda ordem

$\varepsilon$ - $\quad$ Vetor coluna formado com as componentes do tensor de deformações de segunda ordem

$\sigma_{0}-\quad$ Vetor coluna formado com as componentes do tensor de tensões iniciais de segunda ordem

$\varepsilon_{0}$ - Vetor coluna formado com as componentes do tensor de deformações iniciais de segunda ordem

$\boldsymbol{E}$ - $\quad$ Módulo de Young

$v$ - $\quad$ Coeficiente de Poisson

D - $\quad$ Matriz de rigidez para materiais elásticos, lineares e isótropos

$f$ - $\quad$ Matriz de flexibilidade para materiais elásticos, lineares e isótropos

$\boldsymbol{L}$ - $\quad$ Operador diferencial divergente

$\boldsymbol{u}$ - Vetor de deslocamentos

$\overline{\boldsymbol{u}}$ - $\quad$ Vetor de deslocamentos impostos

$\boldsymbol{n}$ - Vetor normal ao contorno

$N$ - $\quad$ Matriz construída com as componentes do vetor normal ao contorno

$\Pi$ - $\quad$ Quantidade escalar (funcional) 
$\bar{\Pi}$ - Quantidade escalar modificada (funcional modificado)

$\boldsymbol{R}, \boldsymbol{M}$ - Operadores diferenciais

$\boldsymbol{u}$ - $\quad$ No Cap.2- item 2.3, função supostamente contínua com derivadas contínuas

$\delta$ - operador variacional

$\boldsymbol{A}(\boldsymbol{u})$ - Equação diferencial num domínio

$\boldsymbol{B}(\boldsymbol{u})$ - Condições de contorno

$\boldsymbol{C}(\boldsymbol{u})$ - Conjunto de restrições expresso por equações diferenciais aplicadas no domínio

$\lambda, \lambda_{1}$ e $\lambda_{2}-$ Multiplicadores de Lagrange

$\boldsymbol{E}(\boldsymbol{u})$ - Conjunto de restrições expresso por equações diferenciais aplicadas no contorno

$\boldsymbol{U}(\varepsilon)$ - Energia de deformação interna

$\boldsymbol{V}(\boldsymbol{u})$ - Energia potencial do carregamento externo

$\boldsymbol{U}(\sigma)^{*}$ - Energia de deformação complementar

$\boldsymbol{u}_{\Gamma-} \quad$ Vetor do deslocamento no contorno

$S_{\Omega}$ - Matriz com as funções de interpolação do campo de tensões

$\boldsymbol{s}_{\Omega}-\quad$ Vetor que agrupa os graus de liberdade do campo de tensões

$\hat{\sigma}$ - $\quad$ Vetor das tensões aproximadas

$\boldsymbol{U}_{\Omega}{ }^{-} \quad$ Matriz com funções de interpolação do campo de deslocamentos no domínio

$q_{\Omega}-\quad$ Vetor que agrupa os graus de liberdade do campo de deslocamentos no domínio

$\hat{\boldsymbol{u}}$ - $\quad$ Vetor dos deslocamentos no domínio aproximados

$\boldsymbol{U}_{\Gamma}$ - $\quad$ Matriz com as funções de interpolação do campo de deslocamentos no contorno

$\boldsymbol{q}_{\Gamma}-\quad$ Vetor que agrupa os graus de liberdade do campo de deslocamentos no contorno

$\hat{\boldsymbol{u}}_{\Gamma}$ - Vetor dos deslocamentos no contorno aproximados

F - $\quad$ Matriz quadrada cujos termos são integrações no domínio sobre as aproximações do campo de tensões no domínio e matriz de flexibilidade

$\boldsymbol{A}_{\Omega}{ }^{-} \quad$ Matriz cujos termos são integrações no domínio sobre as aproximações do campo de tensões e deslocamentos no domínio

$A_{\Gamma}$ - Matriz cujos termos são integrações no contorno sobre as aproximações do campo de tensões no domínio e deslocamento no contorno

$\boldsymbol{e}_{\Gamma}-\quad$ Vetor cujos termos são integrações no contorno sobre as aproximações do campo de tensões no domínio e do vetor de deslocamentos impostos

$\boldsymbol{Q}_{\Omega}-\quad$ Vetor cujos termos são integrações no domínio sobre as aproximações do campo de deslocamentos no domínio e do vetor de forças volúmicas 
$\boldsymbol{Q}_{\Gamma}$ - $\quad$ Vetor cujos termos são integrações no contorno sobre as aproximações do campo de deslocamento no contorno e do vetor de forças de superfície

$\boldsymbol{n}_{\Omega}$ - $\quad$ Malha de elementos finitos (malha de cobertura de domínio)

$\boldsymbol{e} \quad$ Elementos finitos da malha de cobertura de domínio

$\boldsymbol{i} \quad$ Elementos finitos da malha de cobertura de contorno

$\boldsymbol{n}_{\Gamma}-\quad$ Malha de cobertura de contorno

$\Omega_{e}-\quad$ Domínio de um elemento finito, $\boldsymbol{e}=\mathbf{1}, \ldots \boldsymbol{n}_{\Omega}$

$\Gamma_{i}$ - Lados dos elementos que pertencem ao contorno do domínio, $i=1, \ldots n_{\Gamma}$

$\boldsymbol{s}_{\Omega_{e}}-\quad$ Vetor que guarda os graus de liberdade nodais correspondentes às tensões pertencentes ao elemento $\boldsymbol{e}$

$\boldsymbol{q}_{\Omega_{e}}-\quad$ Vetor que guarda os graus de liberdade nodais correspondentes aos deslocamentos no domínio pertencentes ao elemento $\boldsymbol{e}$

$\boldsymbol{q}_{\Gamma_{i}}-\quad$ Vetor que guarda os graus de liberdade nodais correspondentes aos deslocamentos no contorno pertencentes ao elemento $\boldsymbol{i}$

$\boldsymbol{F}_{\mathbf{e}}$ e $\boldsymbol{A}_{\boldsymbol{\Omega}_{\mathbf{e}}}-$ Matrizes Booleanas que extraem os graus de liberdade do elemento finito de domínio

$\mathbf{A}_{\boldsymbol{r}}$ - Matriz Booleana que extrai os graus de liberdade do elemento finito de contorno

$\hat{\sigma}_{e}-\quad$ Vetor das tensões aproximadas (restritas ao domínio de um elemento)

$\boldsymbol{S}_{\Omega_{e}} \quad$ Matriz que coleta as aproximações do campo de tensões no domínio de um elemento

$U_{\Omega_{e}}-\quad$ Matriz que coleta as aproximações do campo de deslocamentos no domínio de um elemento

$U_{\Gamma_{i}}$ - Matriz que coleta as aproximações do campo de deslocamentos no contorno de um elemento

$\boldsymbol{F}_{\boldsymbol{e}}$ - Matriz quadrada cujos termos são integrações no domínio de um elemento sobre as aproximações do campo de tensões no domínio e matriz de flexibilidade

$\boldsymbol{A}_{\Omega e}{ }^{-} \quad$ Matriz cujos termos são integrações no domínio de um elemento sobre as aproximações do campo de tensões e deslocamentos no domínio

$A_{\Gamma i}$ - Matriz cujos termos são integrações no contorno de um elemento sobre as aproximações do campo de tensões no domínio e deslocamento no contorno

$\boldsymbol{Q}_{\Gamma_{i}}-\quad$ Vetor cujos termos são integrações no contorno de um elemento sobre as aproximações do campo de deslocamento no contorno e do vetor de forças de superfície 
$N_{S} \quad$ Número de nós de tensão no domínio

$N_{U} \quad$ Número de nós de deslocamento no domínio

$N_{\Gamma} \quad$ Número de nós de deslocamento no contorno

$\sum_{N_{S}} \quad$ Sub-matriz que reúne as funções interpoladoras dos campos de tensão no domínio

$\boldsymbol{H}_{N_{U}} \quad$ Sub-matriz que reúne as funções interpoladoras dos campos de deslocamento no domínio

$\boldsymbol{G}_{\boldsymbol{N}_{\Gamma}} \quad$ Sub-matriz que reúne as funções interpoladoras dos campos de deslocamento no contorno

$\boldsymbol{I}_{3 \mathrm{e}} \boldsymbol{I}_{2} \quad$ Matrizes identidades de terceira e segunda ordem respectivamente

$\varphi_{\alpha} \quad$ Funções bilineares Lagrangianas para elementos finitos quadrilaterais, $\alpha=1,2,3,4$

$\psi_{\gamma} \quad$ Funções lineares Lagrangianas para os lados dos elementos finitos quadrilaterais que pertencem ao contorno, $\gamma=\mathbf{1 , 2}$

$\boldsymbol{X}_{\alpha}$ e $\boldsymbol{Y}_{\alpha} \quad$ São as coordenadas adimensionais dos nós do elemento quadrilateral, $\alpha=1,2,3,4$

$\xi \quad E$ E a coordenada adimensional com origem no centro do lado do elemento quadrilateral que pertence ao contorno $\Gamma$

$\boldsymbol{Q}_{N} \quad$ Conjunto de nós escolhidos para a construção do modelo aproximado num domínio $\Omega$

$\boldsymbol{x}_{j} \quad$ Nó que compõe o conjunto $\boldsymbol{Q}_{N}, j=1,2, \ldots, N$

$\boldsymbol{N} \quad$ No apêndice B -Número de nós $\boldsymbol{x}_{\boldsymbol{j}}$

$\omega_{j} \quad$ Região de influência, ou nuvem, de um nó $\boldsymbol{x}_{\boldsymbol{j}}$

$\boldsymbol{r}_{j} \quad$ Raio que define a nuvem nodal $\omega_{j}$

$\mathfrak{I}_{N} \quad$ Região formada pela união de todas as nuvens nodais $\omega_{j}$

$\boldsymbol{W}_{j} \quad$ Função ponderadora associada à nuvem $\omega_{j}$

$C_{0}^{q}\left(\omega_{j}\right) \quad$ Conjunto de funções cujas derivadas até a ordem $q$ apresentam suporte compacto

$\tilde{\boldsymbol{u}}_{j} \quad$ Conjunto de dados definido em cada ponto $\boldsymbol{x}_{j}, \boldsymbol{j}=\mathbf{1}, \ldots, N$

$\hat{\boldsymbol{u}}(\boldsymbol{x}) \quad$ Função aproximadora global (MMQM)

$\alpha(x)$ Vetor das constantes da combinação linear que define a aproximação $\hat{\boldsymbol{u}}(\boldsymbol{x})$

$\boldsymbol{p}(\boldsymbol{x}) \quad$ Vetor que reúne a base de funções adotadas na aproximação $\hat{\boldsymbol{u}}(\boldsymbol{x})$

$\Psi_{j}(x) \quad$ Funções de forma associada ao nó $\boldsymbol{x}_{j}$

$\boldsymbol{J}(\boldsymbol{x}) \quad$ Funcional quadrático do erro da aproximação (MMQM)

$\boldsymbol{H}(\boldsymbol{x})$ Matriz utilizada na geração das funções de forma $\Psi_{j}(\boldsymbol{x})$, no MMQM 
$\boldsymbol{G}_{j}(\boldsymbol{x}) \quad$ Vetor utilizado na geração da função de forma $\Psi_{j}(\boldsymbol{x})$, no MMQM

$\boldsymbol{p}_{\boldsymbol{k}} \quad$ Base polinomial de ordem $\boldsymbol{k}$

$\boldsymbol{p}_{p} \quad$ Base polinomial de ordem $\boldsymbol{p}$

$\mathfrak{I}_{N}^{k, p} \quad$ Família de funções do Método das Nuvens hp

$\widetilde{J}_{N}^{p} \quad$ Família de funções para o campo de tensões no domínio

$\boldsymbol{L}_{j n_{e}} \quad \boldsymbol{n}_{e}$ funções que multiplicam ou enriquecem as aproximações do campo de tensões no domínio definida em cada nó de índice $\boldsymbol{j}$

$\boldsymbol{b}_{j i} \quad$ Novos parâmetros nodais em correspondência a cada componente de $S_{\Omega_{j}} L_{j n_{e}}$

$\Theta_{N}^{p} \quad$ Família de funções para o campo de deslocamentos no domínio

$\boldsymbol{M}_{j \boldsymbol{n}_{e}} \quad \boldsymbol{n}_{\boldsymbol{e}}$ funções que multiplicam ou enriquecem as aproximações do campo de deslocamentos no domínio definida em cada nó de índice $\boldsymbol{j}$

$\boldsymbol{c}_{j i} \quad$ Novos parâmetros nodais em correspondência a cada componente de $\boldsymbol{U}_{\Omega_{j}} \boldsymbol{M}_{\boldsymbol{j} \boldsymbol{n}_{e}}$

$\Xi_{N}^{p} \quad$ Família de funções para o campo de deslocamentos no contorno

$\boldsymbol{O}_{j n_{e_{\Gamma}}} \quad \boldsymbol{n}_{e_{\Gamma}}$ funções que multiplicam ou enriquecem a função de forma de deslocamento no contorno definida em cada nó de índice $\boldsymbol{j}$;

$\boldsymbol{d}_{j i} \quad$ Novos parâmetros nodais em correspondência a cada componente de $\boldsymbol{U}_{\Gamma_{j}} \boldsymbol{O}_{j n_{e_{\Gamma}}}$

$I(j)$ Contador para o número de funções adicionadas a cada nó de índice $j$

$\boldsymbol{h}_{k \alpha} \quad$ Funções polinomiais que enriquecem um nó $\alpha$ de domínio, $k \alpha=1, \ldots, n_{e}$

$\boldsymbol{h}_{k \alpha_{\Gamma}} \quad$ Funções polinomiais que enriquecem um nó $\alpha_{\Gamma}$ de contorno, $\boldsymbol{k} \alpha_{\Gamma}=1, \ldots, n_{e_{\Gamma}}$

$\Delta_{\alpha} \quad$ Matriz de enriquecimento polinomial do nó $\alpha$ no domínio, $\alpha=1, \ldots, 4$

$\Delta_{\Gamma_{\alpha_{\Gamma}}} \quad$ Matriz de enriquecimento polinomial do nó $\alpha_{\Gamma}$ no contorno, $\alpha_{\Gamma}=\mathbf{1 , 2}$

$\boldsymbol{g}_{a p} \quad$ Grau máximo dos polinômios $\boldsymbol{h}_{\boldsymbol{k} \alpha}$

$\boldsymbol{g}_{a p \Gamma} \quad$ Grau máximo dos polinômios $\boldsymbol{h}_{\boldsymbol{k} \alpha_{\mathrm{T}}}$

r Distância de um ponto até a ponta de uma fissura

$\theta \quad$ Ângulo entre o vetor posição do ponto que define a distância $\mathbf{r}$ e o eixo $x$

$\sigma_{x}, \sigma_{y}$ e $\tau_{x y}$ No capítulo 5, item 5.3.3 - distribuição das tensões elásticas próximo a ponta de uma fenda

$\boldsymbol{u} \boldsymbol{e} \boldsymbol{v} \quad$ No capítulo 5, item 5.3.3 Campo de deslocamentos próximo à ponta de uma fenda

$\sigma_{x}^{\prime}, \sigma_{y}^{\prime}$ e $\tau_{x y}^{\prime}$ No capítulo 5, item 5.3.3 - funções que se assemelham à distribuição das tensões elásticas próximo a ponta de uma fenda

$\boldsymbol{u}^{\prime} \boldsymbol{e} \boldsymbol{v}^{\prime} \quad$ No capítulo 5, item 5.3.3 funções que se assemelham ao campo de deslocamentos próximo à ponta de uma fenda 


\begin{tabular}{|c|c|}
\hline $\boldsymbol{G}$ & Módulo de elasticidade transversal \\
\hline $\mathbf{k}$ & $\begin{array}{l}\text { Variável definida no campo de deslocamentos próximo a ponta de } \\
\text { uma fenda }\end{array}$ \\
\hline$K_{I}$ & Fator de intensidade de tensão \\
\hline$\sigma_{\alpha}$ & $\begin{array}{l}\text { Matriz de enriquecimento do campo de tensões no nó } \alpha \text { de domínio, } \\
\alpha=1, \ldots, 4\end{array}$ \\
\hline $\boldsymbol{u}_{\alpha}$ & $\begin{array}{l}\text { Matriz de enriquecimento do campo de deslocamentos no nó } \alpha \text { de } \\
\text { domínio, } \alpha=1, \ldots, 4\end{array}$ \\
\hline$A(x, y)$ & Função de Airy \\
\hline$\sigma_{x}, \sigma_{y}$ e $\tau_{x y}$ & No capítulo 5 , item 5.3.4- campo de tensões obtidas de $A(x, y)$ \\
\hline$A_{\alpha}$ & $\begin{array}{l}\text { Matriz de enriquecimento polinomial do campo de tensões no nó } \alpha \text { de } \\
\text { domínio, } \alpha=1, \ldots, 4\end{array}$ \\
\hline$d_{e}$ & Grau de enriquecimento com os polinômios auto-equilibrados \\
\hline $\boldsymbol{E}_{d_{e} \alpha}$ & Matriz de monômios obtidas de $A(x, y)$ \\
\hline$n_{e}$ & $\begin{array}{l}\text { Número de funções que enriquecem a base aproximativa inicial dos } \\
\text { campos de tensão no domínio }\end{array}$ \\
\hline $\begin{array}{l}f_{11}, f_{12}, f_{21}, f_{22} \\
\quad \text { e } f_{33}\end{array}$ & Termos da matriz de flexibilidade \\
\hline$A, B, C$ e $D$ & $\begin{array}{l}\text { No capítulo 6, matrizes utilizadas no desenvolvimento do Teste do } \\
\text { Mosaico }\end{array}$ \\
\hline$x$ e $y$ & No capítulo 6, vetores de dimensões $\boldsymbol{n}_{\boldsymbol{x}}$ e $\boldsymbol{n}_{\boldsymbol{y}}$, respectivamente \\
\hline $\boldsymbol{n}_{\boldsymbol{x}}$ e $\boldsymbol{n}_{\boldsymbol{y}}$ & Número de variáveis de dos vetores $\boldsymbol{x}$ e $\boldsymbol{y}$, respectivamente \\
\hline $\mathrm{H}_{1}$ e $\mathrm{H}_{2}$ & Espaços de Hilbert \\
\hline$B(.,)$. & Forma bilinear $B(.,$.$) definida em \boldsymbol{H}_{1} \boldsymbol{X} \boldsymbol{H}_{2}$ \\
\hline $\boldsymbol{F}$ & Funcional linear definido em $\boldsymbol{H}_{2}$ \\
\hline$\delta W_{e x t}$ & Trabalho virtual externo \\
\hline$\delta U$ & Trabalho virtual interno \\
\hline$u_{0}$ & Solução do problema variacional \\
\hline$v$ & No capítulo 8 , item 8.2 - deslocamento virtual \\
\hline $\boldsymbol{P}^{2}(\Omega)$ & Espaço de Sobolev \\
\hline$\|\cdot\|_{H_{1}} \mathrm{e}\|\cdot\|_{H_{2}}$ & Normas definidas no espaço de Hilbert \\
\hline$\|\cdot\|_{P^{2}(\Omega)}$ & Norma definida no espaço de Sobolev \\
\hline$S_{1}^{n}$ e $\boldsymbol{S}_{2}^{n}$ & Espaços lineares $n$-dimensionais \\
\hline$u_{n}$ & solução aproximada do problema variacional \\
\hline inf & Valor ínfimo \\
\hline sup & Valor supremo \\
\hline$\lambda(n)$ & Constante positiva que é o valor da condição (inf-sup) \\
\hline$A_{1} e A_{2}$ & Matrizes simétricas positivas \\
\hline$x \boldsymbol{e} \mu$ & $\begin{array}{l}\text { No capítulo 8, item } 8.2 .2 \text { são autovetores e autovalores, } \\
\text { respectivamente }\end{array}$ \\
\hline
\end{tabular}




\section{Lista de Tabelas}

Tabela 1.1 - Características da FHMT, FHT e FHTFT.......................................... 4

Tabela 2.1 - Classificação do Método dos Elementos Finitos na Mecânica dos Sólidos.

Tabela 7.1 - Resultados gerais obtidos para o problema 1 com malha regular $8 \times 9$ e função enriquecedora do tipo $\left(y-y_{n o ́}\right)^{2}$

Tabela 7.2 - Resultados gerais obtidos para o problema 1 com malha regular 8x9 e função enriquecedora do tipo $\left(y-y_{n o ́}\right)$.

Tabela 7.3 - Deslocamentos do nó de referência da malha de cobertura de domínio e contorno para a malha regular $8 \times 9$ e função enriquecedora do tipo $\left(y-y_{n o}\right)^{2}$

Tabela 7.4 - Resultados gerais obtidos para o problema 1 com malha regular 8x9 e função de Airy enriquecedora do tipo polinomial $\left(d_{e}=1\right)$.

Tabela 7.5 - Resultados gerais obtidos para o problema 1 com malha regular $8 \times 9$ e função de Airy enriquecedora do tipo polinomial $\left(d_{e}=2\right)$.

Tabela 7.6 - Resultados gerais obtidos para o problema 1 com malha irregular $12 \times 15$

Tabela 7.7 - Deslocamentos do nó de referência da malha de cobertura de domínio e contorno para a malha irregular $12 \times 15$.

Tabela 7.8 - Resultados gerais obtidos para o problema 1 com malha regular $12 \times 15$ e função de Airy enriquecedora do tipo polinomial $\left(d_{e}=1\right)$

Tabela 7.9 - Resultados gerais obtidos para o problema 1 com malha regular $12 \times 15$ e função de Airy enriquecedora do tipo polinomial $\left(d_{e}=2\right)$.

Tabela 7.10 - Resultados gerais obtidos para o problema 1 com malha irregular $12 \times 15$ e o enriquecimento realizado com funções trigonométricas.

Tabela 7.11 - Deslocamentos do nó de referência da malha de cobertura de domínio e contorno para a malha irregular $12 \times 15$

Tabela 7.12 - Resultados gerais do problema 2 obtidos com a malha regular $3 \times 3$ para algumas situações com enriquecimento polinomial.

Tabela 7.13 - Resultados gerais do problema 2 obtidos com a malha regular $3 \times 3$ para algumas situações com enriquecimento não polinomial. 
Tabela 7.14 - Resultados gerais do problema 2 obtidos com a malha irregular $12 \times 9$ para algumas situações com enriquecimento polinomial........

Tabela 7.15 - Deslocamentos do nó de referência da malha de cobertura de domínio e contorno para a malha irregular $12 \times 9$ com enriquecimento polinomial.

Tabela 7.16 - Resultados gerais do problema 2 obtidos com a malha irregular 12x9 para algumas situações com enriquecimento não polinomial

Tabela 7.17 - Resultados gerais obtidos para o problema 2 com malha regular $12 \times 9$ e função de Airy enriquecedora do tipo polinomial $\left(d_{e}=1\right)$

Tabela 7.18 - Resultados gerais obtidos para o problema 2 com malha irregular $12 \times 9$ e função de Airy enriquecedora do tipo polinomial $\left(d_{e}=2\right)$

Tabela 7.19 - Deslocamentos do nó de referência da malha de cobertura de domínio e contorno para a malha irregular $12 \times 9$ e função de Airy enriquecedora do tipo polinomial $\left(\boldsymbol{d}_{e}=2\right)$.

Tabela 7.20 - Resultados gerais obtidos para o problema 2 com malha irregular $12 \times 9$ e o enriquecimento realizado com funções trigonométricas..

Tabela 7.21 - Deslocamentos do nó de referência da malha de cobertura de domínio e contorno para a malha irregular $12 \times 15$. 


\section{ABREVIATURAS}

MEF - $\quad$ Método dos Elementos Finitos

MEFG - Método dos Elementos Finitos Generalizados

FHMT - Formulação Híbrida-Mista de Tensão

FHT - $\quad$ Formulação Híbrida de Tensão

FHTFT - Formulação Híbrida-Trefftz de Tensão

PVC - $\quad$ Problema de Valor de Contorno

MMQM - Método dos Mínimos Quadrados Móveis

PTV - Princípio dos Trabalhos Virtuais

MED - $\quad$ Método dos Elementos Difusos

MGLE - Método de Galerkin Livre de Elementos

MPF - Método dos Pontos Finitos

PU - Partição da Unidade

MEFPU - Método dos Elementos Finitos da Partição da Unidade 


\section{SUMÁRIO}

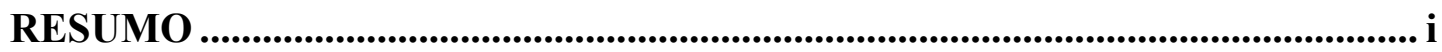

ABSTRACT ..................................................................................................................... ii

LISTA DE FIGURAS ............................................................................................... iii

LISTA DE SÍMBOLOS................................................................................................... vii

LISTA DE TABELAS ............................................................................................... xiii

ABREVIATURAS.................................................................................................... $\mathrm{xV}$

1. INTRODUÇÃ

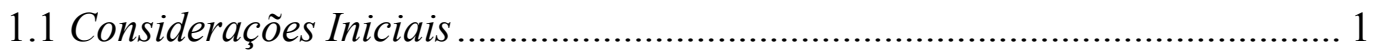

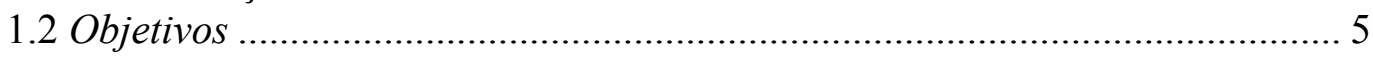

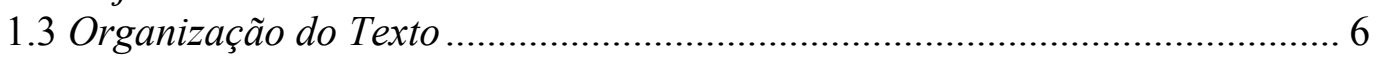

2. PRINCÍPIOS VARIACIONAIS MISTOS ......................................................... 7

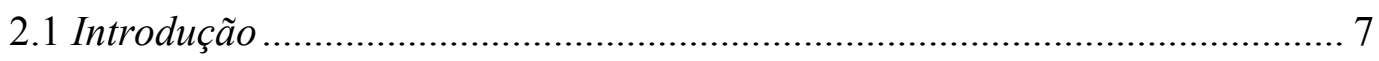

2.2 Conceitos da Teoria da Elasticidade Linear ................................................ 7

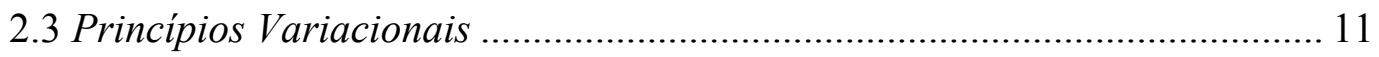

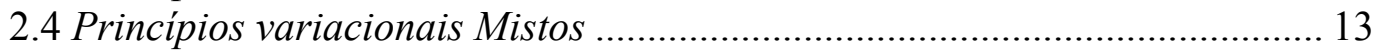

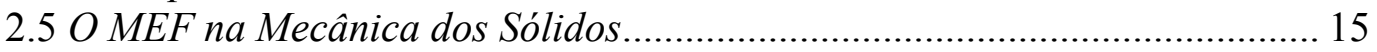

\section{FORMULAÇÃO HÍBRIDA-MISTA DE TENSÃO PARA}

ELASTICIDADE ........................................................................................................... 17

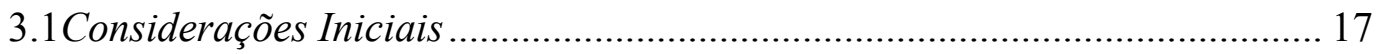

3.2 Formulação Geral Hibrida-Mista de Tensão ................................................. 18

3.3 Forma Aproximada do Modelo Hibrido-Misto de Tensão ............................. 20

3.3.1 Aproximação do Campo de Tensões no Domínio .................................... 20

3.3.2 Aproximação do Campo de Deslocamentos no Domínio .......................... 20

3.3.3 Aproximação do Campo de Deslocamentos no Contorno ........................ 20

3.3.4 Aproximação Geral do Modelo Híbrido-Misto ....................................... 20

\section{FORMULAÇ̃̃O GERAL HÍBRIDA-MISTA}

COM MALHA DE COBERTURA …………………......................................... 23

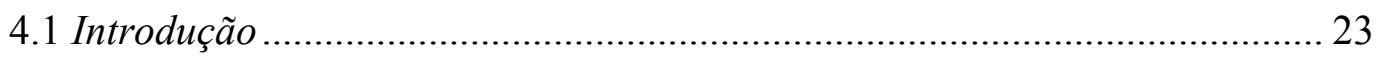

4.2 Modelo Hibrido Misto com Malha de Cobertura .......................................... 23

4.3 Elementos Finitos Híbridos-Mistos Quadrangulares Bilineares................... 26 


\section{FHMT COM ENRIQUECIMENTO - MÉTODO DOS ELEMENTOS FINITOS GENERALIZADOS (MEFG)}

5.1 O Método dos Elementos Finitos Generalizados (MEFG) ........................... 33

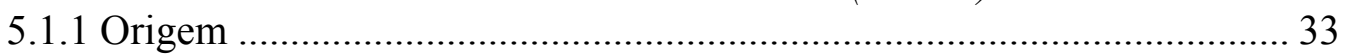

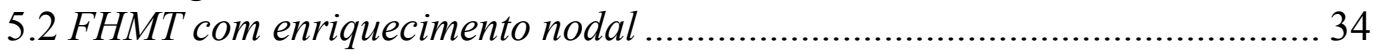

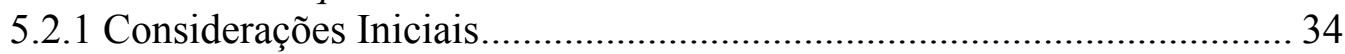

5.3 Elementos Finitos Hibridos-Mistos de Tensão Quadrangulares

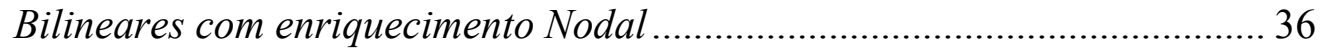

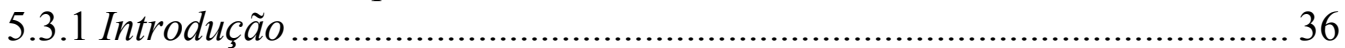

5.3.2 Enriquecimento Nodal Polinomial........................................................ 37

5.3.3 Enriquecimento Nodal com Funções que se Assemelham às Soluções da Mecânica da Fratura Elástica Linear...................................................... 42

5.3.4 Enriquecimento Nodal com Campo de Tensões Auto-equilibrados ........ 47

5.3.5 Enriquecimento com Funções Trigonométricas ..................................... 56

\section{ESTUDO INICIAL DAS CONDIÇÕES DE CONVERGÊNCIA DA FHMT} COM ENRIQUECIMENTO NODAL ............................................................58

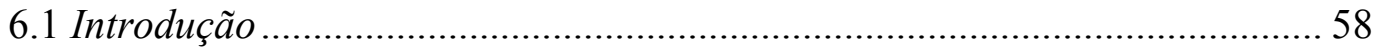

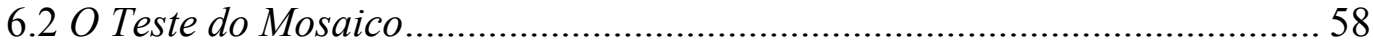

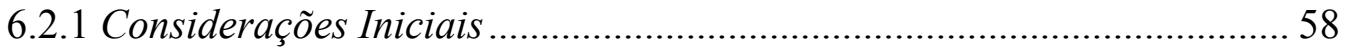

6.2.2 O Teste do Mosaico ...................................................................................... 59

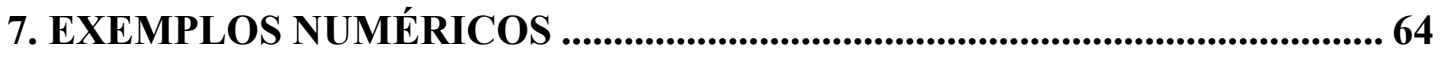

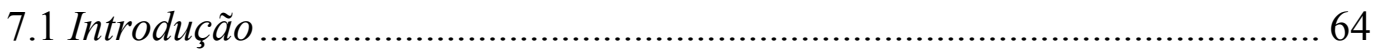

7.2 Problema 1: Chapa Tracionada Simetricamente .......................................... 67

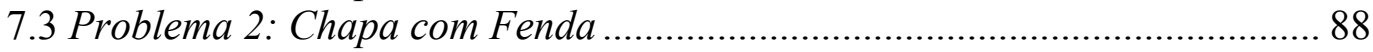

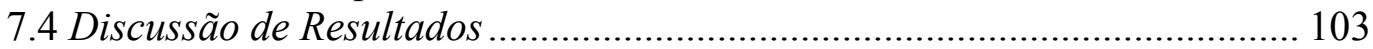

8. ESTUDO DA CONDIÇÃO DE BABUŠKA-BREZZI (INF-SUP) APLICADA À FHMT ................................................................ 108

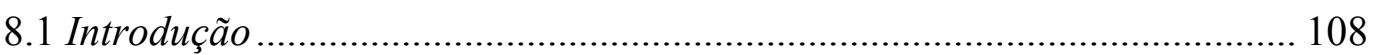

8.2 Problema Elástico Linear em Deslocamentos - Método dos

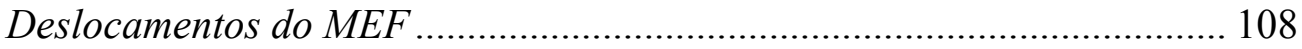

8.2.1 Problema Bem Colocado ......................................................................... 111

8.2.2 Determinação Numérica de $\boldsymbol{\lambda}(\boldsymbol{n})$ para uma Formulação em

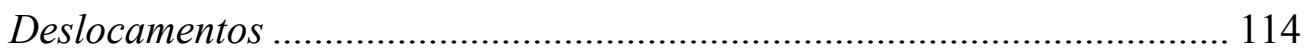

8.3 A Condição de Babuška-Brezzi (inf-sup) Aplicada à FHMT.........................116

8.3.1 O Teste Numérico da Condição de Babuška-Brezzi (inf-sup) Aplicada à FHMT.

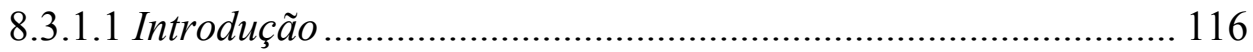

8.3.1.2 O inf-sup Teste Aplicado a Problemas Incompressíveis ............ 117

8.3.1.3 O inf-sup Teste Aplicado à FHMT com Enriquecimento ........... 117

8.4 Discussões sobre a Condição de Babuška-Brezzi (inf-sup) aplicada à FHMT com Enriquecimento Nodal.. 
9. CONCLUSÕES E CONSIDERAÇÕES FINAIS ......................................... 125 REFERÊNCIAS BIBLIOGRÁFICAS E BIBLIOGRAFIA BÁSICA .............. 129 APÊNDICE A

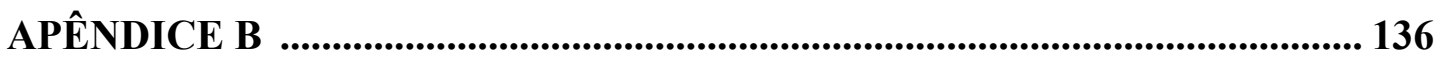

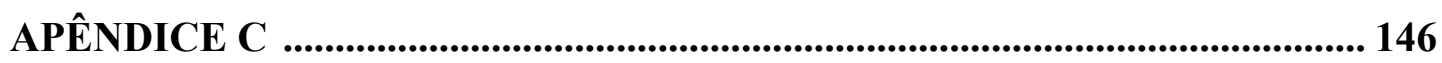

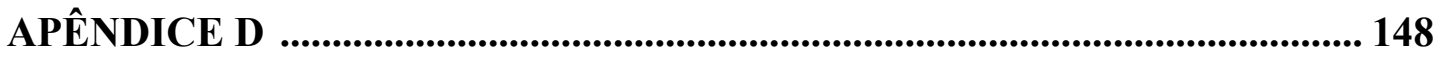

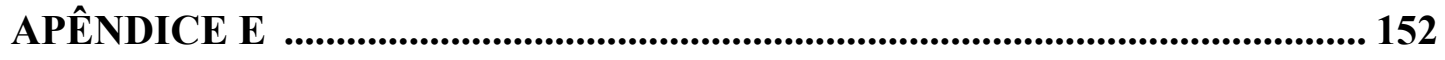




\section{Introdução}

\subsection{Considerações Iniciais}

O Método dos Elementos Finitos (MEF) é um dos métodos numéricos mais difundidos na comunidade técnica e científica e tornou-se ferramenta usual para análise de problemas em engenharia. Particularmente, na mecânica estrutural, a chamada "formulação em deslocamentos" do MEF é preferencialmente estudada. Esse "sucesso", em parte, deve-se à sua simplicidade conceitual e facilidade de implementação computacional.

Mas a "formulação em deslocamentos" decorre, na verdade, da forma como é expresso o Problema de Valor de Contorno (PVC), a qual é independente do Método dos Elementos Finitos (MEF). Neste trabalho o MEF é entendido como uma técnica sistemática de geração de funções interpoladoras que podem ser utilizadas nas diferentes formas nas quais o PVC é expresso, com o objetivo de gerar uma solução aproximada. As formas ditas variacionais estão associadas a um princípio variacional (como o Princípio da Mínima Energia Potencial, Princípio dos Trabalhos Virtuais, Princípio da Mínima Energia Complementar, etc.) ou à ponderação da forma forte do modelo estudado.

Apesar de bastante usado, o modelo "em deslocamentos" do MEF, quando é baseado em aproximação polinomial de baixa ordem da solução, apresenta limitações, particularmente decorrentes da crescente perda de precisão nas ordens superiores de derivadas da função aproximativa. $\mathrm{Na}$ análise de problemas da elasticidade, por exemplo, a solução final do campo de tensão, embora seja a variável mais importante em alguns casos, pode apresentar baixa precisão, pois depende de derivadas da solução em deslocamentos. Do ponto de vista numérico, diferenciação implica em perda de precisão. 
Outra limitação que pode ocorrer é o fenômeno denominado de travamento (“locking”). Trata-se, essencialmente, da redução na capacidade de aproximação de um elemento finito, em conseqüência de uma restrição imposta pelo modelo teórico. Especificamente nos estudos de cascas, placas e sólidos aonde se impõe a restrição de incompressibilidade sobre as deformações plásticas, por exemplo, a formulação em deslocamentos do MEF pode apresentar essa dificuldade numérica, (BATHE, 1996).

Por outro lado, em problemas que apresentam regiões com singularidades (por exemplo, que se caracterizam por elevados gradientes de tensão) a taxa de convergência exponencial, usualmente obtida com as funções de forma polinomiais geradas pelo MEF clássico, não é mais observada, (SZABÓ; BABUŠKA, 1991). Assim, uma melhor representação do campo de tensões fornecida pelo modelo convencional do MEF passa a exigir uma boa estratégia de refinamento, aliada a um número considerável de elementos finitos na discretização dessa região singular.

Essa última situação, procedimento típico da versão h-adaptativa do MEF clássico, muitas vezes torna-se inviável, pois o custo computacional pode ser demasiadamente elevado. Além desse entrave, muitos geradores de malhas, utilizados nas análises numéricas, não conseguem limitar a partição do domínio somente à região próxima à singularidade, mas acabam criando refinamentos desnecessários, ou elementos distorcidos, em outras partes do domínio. Segundo Duarte, Babuška e Oden (2000), esta prática pode fornecer uma convergência de resultados insatisfatória, mesmo em pontos do domínio distantes da singularidade.

Em análise de problemas com grandes deformações, com a utilização do MEF convencional, é necessária uma mudança contínua da malha para evitar distorções exageradas nos elementos. Para estudo destes últimos casos, com o uso dos processos padaptativo ou hp-adaptativo do MEF clássico as dificuldades podem ser ainda maiores.

Formulações não-convencionais do PVC vem sendo desenvolvidas com o objetivo de melhorar a precisão das análises onde a forma do MEF em deslocamentos não consegue apresentar bons resultados, com baixo custo computacional. Dentre as formulações não-convencionais do PVC, destacam-se as formulações híbridas mistas de tensão e de deformação, onde os campos de tensão e deformação, respectivamente, também podem ser diretamente aproximados.

As formulações em tensão se dividem basicamente em três tipos: Híbrida, Híbrida-Trefftz e Híbrida-Mista. A formulação Híbrida-Mista de Tensão (FHMT), foco principal do trabalho, está apresentada, por exemplo, em Freitas, Almeida e Pereira 
(1996). Esta formulação tem como principais vantagens a possibilidade de aproximação independente das variáveis em deslocamentos e tensão, envolvidas no PVC, e a escolha de diferentes graus na aproximação dos campos envolvidos na análise.

Essa forma não-convencional do MEF é denominada mista porque se fundamenta na aproximação direta de dois campos incompatíveis no domínio: designados de tensões e de deslocamentos. Como os deslocamentos também são aproximados no contorno, onde as forças de superfícies são impostas, a formulação também é caracterizada como híbrida. O modelo utilizado diz-se de tensão, pois a formulação é desenvolvida de modo a permitir, sob certas condições, a determinação de soluções estaticamente admissíveis, isto é, soluções que satisfazem localmente as condições de equilíbrio de domínio e de contorno do problema.

O modelo Híbrido de Tensão (FHT) pode ser obtido do modelo Híbrido-Misto de Tensão pela simples escolha de funções interpoladoras do campo de tensão que satisfazem previamente a equação diferencial de equilíbrio local. Esta condição vem a evidenciar a redundância da equação de equilíbrio e, por conseqüência, o campo de deslocamentos no domínio também passa a ser redundante.

Assim, no modelo Híbrido de Tensão, têm-se somente aproximações dos campos de tensão no domínio e de deslocamento no contorno.

$\mathrm{Na}$ formulação Híbrida-Trefftz de Tensão (FHTFT), tem-se também a aproximação dos campos de tensão no domínio e o deslocamento no contorno do problema. Como características das funções aproximativas dos campos de tensão é que estas devem ser derivadas de potenciais de tensões bi-harmônicos. Como citado no trabalho de Bussamra (1999), na formulação Híbrida de Tensão e Híbrida-Trefftz de Tensão não existe interpolação nodal. Dessa forma, os nós de um elemento não têm muita importância, pois as aproximações dos campos de tensão não estão atreladas a eles. Outra grande característica dessas duas formulações é que as integrações do modelo discreto podem ser realizadas no contorno do elemento.

Para essas três formulações onde os campos de tensão são diretamente aproximados, os sistemas de equações lineares são esparsos e simétricos. Resumem-se na tabela 1.1, as formulações onde os campos de tensão são diretamente aproximados e as suas principais características: 
Tabela 1.1 - Características da FHMT, FHT e FHTFT.

\begin{tabular}{ccc}
\hline Formulação & Campos Aproximados & $\begin{array}{c}\text { Restrição Sobre os Campos } \\
\text { Aproximados }\end{array}$ \\
\hline FHMT & $\begin{array}{c}\text { Tensão e deslocamento no } \\
\text { domínio e deslocamento no } \\
\text { contorno }\end{array}$ & Nenhuma \\
\hline FHT & Tensão no domínio e \\
& deslocamento no contorno & $\begin{array}{c}\text { As aproximações para o campo } \\
\text { de tensões devem satisfazer a } \\
\text { equação diferencial de equilíbrio }\end{array}$ \\
\hline \multirow{2}{*}{ FHTFT } & Tensão no domínio e & $\begin{array}{c}\text { As aproximações para o campo } \\
\text { de tensões devem satisfazer a } \\
\text { restrição de Trefftz }\end{array}$ \\
\hline
\end{tabular}

Por outro lado, segundo Duarte (1995), os métodos sem malha são definidos como métodos numéricos para solução de problemas de valor de contorno (PVC), onde as equações que governam o modelo discreto independem totalmente ou em parte, do conceito de uma malha de elementos finitos. Em Duarte (1995), encontra-se também uma revisão geral sobre os métodos sem malha.

No Método das Nuvens "hp" ("hp" Clouds Method), apresentado nos trabalhos de Duarte e Oden (1995), Duarte e Oden (1996), a construção do modelo discreto é gerada a partir de pontos nodais dispersos no domínio, sem nenhuma conectividade. A principal característica desse método é que o enriquecimento das funções de aproximação é realizado sem a necessidade de introduzir novos pontos nodais ao domínio.

Como na maioria dos métodos sem malhas, as funções aproximadoras do Método das Nuvens hp são determinadas pelo Método dos Mínimos Quadrados Móveis (MMQM), (LANCASTER; SALKAUSKAS, 1981,1990). Tais funções de forma constituem uma Partição da Unidade (PU) (essencialmente um conjunto de funções cuja soma, num ponto, é igual à unidade).

No Método dos Elementos Finitos Generalizados (MEFG) (BABUŠKA; CALOZ; OSBORN, 1994; BABUŠKA; MELENK, 1997; DUARTE, 1996; DUARTE; ODEN, 1995, 1996a, 1996b; MELENK; BABUŠKA, 1996; ODEN; DUARTE; ZIENKIEWICZ, 1998), uma malha de cobertura é empregada para definir nuvens suporte dentro das quais as funções de forma do MEF clássico são usadas como PU. As funções de forma podem então ser enriquecidas dentro de cada nuvem, mediante a multiplicação da PU por funções de interesse. 
Os elementos finitos híbrido-mistos também podem ser utilizados como malha de cobertura e as suas funções de forma são usadas como PU. Pimenta, Proença e Freitas (2002) aplicaram o MEFG à FHMT. Como nessa são aproximados independentemente os campos de deslocamentos e tensões, a técnica de enriquecimento nodal também pode ser aplicada de forma independente aos campos de tensões e deslocamentos.Além disso, o conhecimento prévio da solução do problema estudado também pode ser utilizado como função enriquecedora.

Portanto, as muitas potencialidades apresentadas pela FHMT combinada com a técnica de enriquecimento nodal, justificam a continuidade das pesquisas nesse tema.

\subsection{Objetivos}

O trabalho proposto tem por objetivo oferecer uma contribuição ao estudo de uma formulação variacional não-convencional do PVC, denominada de FHMT, combinada com uma técnica de enriquecimento nodal de aproximações definidas por uma malha de elementos finitos.

A pesquisa tem por base o trabalho de Pimenta, Proença e Freitas (2002), que trata da aplicação do MEFG na formulação FHMT. Como primeira extensão do programa fonte desenvolvido naquele estudo, tem-se a possibilidade de enriquecimento de aproximações nodais para o contorno do problema. Esta consideração amplia as possibilidades de combinação das situações de enriquecimento das funções de forma básicas utilizadas, naquele trabalho, nas aproximações dos campos de tensões e deslocamentos no domínio.

Uma segunda extensão é o uso de funções não-polinomiais no enriquecimento da aproximação nodal. No caso de exemplos que apresentem singularidades no campo de tensões, empregam-se, no enriquecimento, aproximações que se assemelham à soluções típicas da mecânica da fratura.

Outra extensão do programa fonte a ser analisada é a possibilidade de enriquecimento das bases aproximativas do campo de tensões com os polinômios que fornecem distribuições de tensões auto-equilibradas.

Um objetivo complementar, preliminarmente desenvolvido neste trabalho, diz respeito à análise da convergência da solução pela verificação da condição de BabuškaBrezzi (BABUŠKA, 1971; BREZZI, 1974). 


\subsection{Organização do Texto}

O conteúdo do trabalho está organizado como se segue:

- No capítulo 2, após uma breve revisão dos conceitos básicos da teoria da elasticidade utilizados no trabalho, apresentam-se os princípios variacionais mistos, que são a base para o desenvolvimento da formulação em elementos finitos estudada.

- No capítulo 3 é descrita a FHMT para a elasticidade. São definidas as formas aproximadas para cada um dos campos envolvidos na FHMT, bem como o sistema linear de equações discretas que governam o modelo.

- O capítulo 4 trata da aplicação do MEF à FHMT, adotando-se o elemento isoparamétrico quadrilateral de quatro nós. São definidas as bases aproximativas utilizadas para aproximação das tensões e deslocamentos no domínio e deslocamentos no contorno do problema. Desenvolvem-se todas as matrizes envolvidas no sistema de equações lineares discretas da FHMT. Também são detalhadas as integrações de cada uma dessas matrizes.

- O capítulo 5 apresenta a aplicação do MEFG sobre a FHMT. Abordam-se, de forma simplificada, as origens do MEFG. Faz-se a definição das novas famílias de funções para os campos de domínio e contorno enriquecidos à maneira do Método das Nuvens "hp". Destaca-se o conjunto de matrizes intervenientes no modelo Híbrido-Misto para as várias situações de enriquecimento propostas no trabalho.

- No capítulo 6, estudam-se, inicialmente, as condições para a solvibilidade do sistema de equações lineares discretas do modelo Híbrido-Misto de Tensão com enriquecimento.

- No capítulo 7 são apresentados os exemplos numéricos e os resultados são discutidos.

- No capítulo 8, apresenta-se também um estudo preliminar sobre a condição de Babuška-Brezzi aplicada à FHMT.

- No capítulo 9 são apresentadas as considerações finais e conclusões. 


\section{Princípios Variacionais Mistos}

\subsection{Introdução}

Neste capítulo é feita inicialmente uma breve revisão dos conceitos essenciais da Teoria da Elasticidade Linear, com o objetivo principal de apresentar a notação adotada. Em seguida, apresentam-se os princípios variacionais mistos, que são a base para o desenvolvimento da FHMT.

\subsection{Conceitos da Teoria da Elasticidade Linear}

As equações aqui apresentadas referem-se a problemas regidos pela Teoria da Elasticidade Linear, dentro dos limites das deformações infinitesimais e pequenos deslocamentos. Não é objetivo deste item esgotar todo o equacionamento e peculiaridades da teoria, os quais podem ser encontrados em obras como Xu (1992), Timoshenko e Goodier (1980) e Valliappan (1981).

Neste trabalho, considera-se o material como um meio elástico com isotropia. Para a apresentação das relações de interesse, será usada a notação matricial e em alguns momentos a notação indicial de Einstein.

Considere-se um corpo elástico ocupando uma região, ou domínio $\Omega$, limitado por um contorno regular $\Gamma$. O sólido é submetido a forças de volume, representadas pelo vetor $\boldsymbol{b}$, distribuídas em $\Omega$ e por forças de superfície, dados pelo vetor $\overline{\boldsymbol{t}}$, distribuídas na parte $\Gamma_{t}$ do contorno. Denotam-se $\Gamma_{u}$ e $\Gamma_{t}$ as partes de $\Gamma$ onde são impostos os deslocamentos e as forças de superfície, respectivamente (ver figura 2.1). Essas duas partes são complementares, ou seja:

$$
\Gamma=\Gamma_{u} \cup \Gamma_{t}
$$

$\mathrm{e}$

$$
\Gamma_{u} \cap \Gamma_{t}=\varnothing
$$




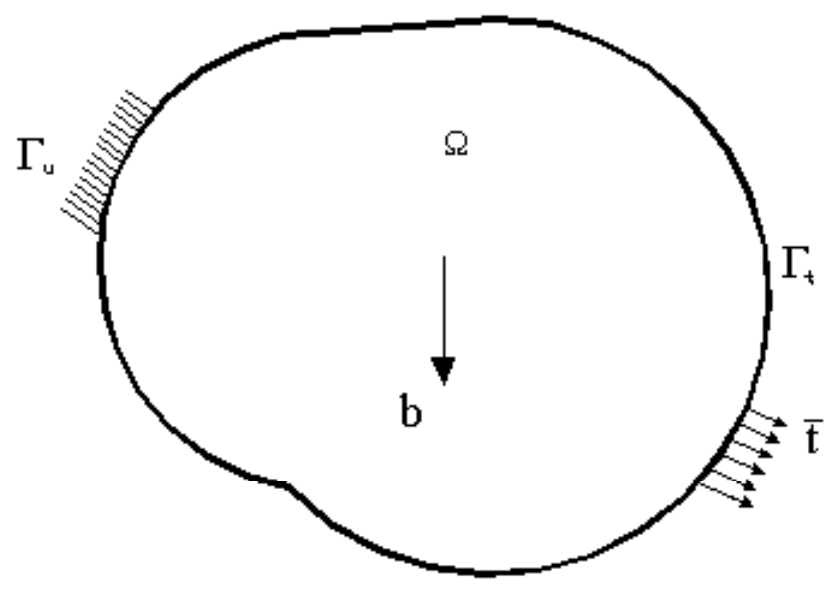

Figura 2.1 - Corpo elástico submetido a forças de volume e de superfície.

Observa-se que os pontos da superfície do contorno do corpo não pertencem ao seu domínio $\Omega$. Assim:

$$
\Omega \cap \Gamma=\varnothing
$$

As equações gerais do problema elástico linear podem ser resumidas da seguinte forma:

- Lei Constitutiva:

$$
\sigma_{i j}=D_{i j k l}\left(\varepsilon_{k l}-\varepsilon_{i j}^{0}\right)+\sigma_{i j}^{0},
$$

ou

$$
\varepsilon_{i j}=f_{i j k l}\left(\sigma_{k l}-\sigma_{i j}^{0}\right)+\varepsilon_{i j}^{0}
$$

(com $i, j, k, 1=1,2,3)$.

Nas eq.(2.1) e eq.(2.2), observa-se que $\boldsymbol{D}_{i j k l}$ e $\boldsymbol{f}_{i j k l}$ são, respectivamente, os tensores constitutivos de rigidez e de flexibilidade de quarta ordem; $\sigma_{i j}$ é o tensor de tensões de segunda ordem; $\varepsilon_{i j}$ é o tensor das deformações de segunda ordem, $\sigma_{i j}^{0}$ e $\varepsilon_{i j}^{0}$ são tensores de segunda ordem de tensão e deformação iniciais, respectivamente. 
- Equações de Equilíbrio:

$$
\sigma_{j i, j}+b_{i}=\mathbf{0}
$$

onde $\boldsymbol{b}_{\boldsymbol{i}}$ é o vetor das forças de volume.

- Equação de Compatibilidade:

$$
\varepsilon_{i j}=\frac{1}{2}\left(u_{i, j}+u_{j, i}\right)
$$

Vale ressaltar que a estas equações acrescentam-se as condições de força/deslocamento prescritas no contorno.

Para o caso de estados planos, as equações anteriores podem ser expressas da seguinte forma:

- Lei Constitutiva:

$$
\begin{aligned}
& \sigma=D\left(\varepsilon-\varepsilon_{0}\right)+\sigma_{0} \\
& \varepsilon=f\left(\sigma-\sigma_{0}\right)+\varepsilon_{0}
\end{aligned}
$$

onde:

$$
\sigma=\left\{\begin{array}{l}
\sigma_{x} \\
\sigma_{y} \\
\tau_{x y}
\end{array}\right\} ; \varepsilon=\left\{\begin{array}{l}
\varepsilon_{x} \\
\varepsilon_{y} \\
\gamma_{x y}
\end{array}\right\} ; D=\frac{E}{1-v^{2}}\left[\begin{array}{ccc}
1 & v & 0 \\
v & 1 & 0 \\
0 & 0 & (1-v) / 2
\end{array}\right] ; f=\frac{1}{E}\left[\begin{array}{ccc}
1 & -v & 0 \\
-v & 1 & 0 \\
0 & 0 & 2(1+v)
\end{array}\right]
$$

onde $\boldsymbol{E}$ é o módulo de elasticidade e $v$ é o coeficiente de Poisson. Nessa notação, $\sigma$ e $\varepsilon$ são representados por vetores coluna formados com as componentes dos tensores de tensão e deformação em um sistema cartesiano de coordenadas $x$ e $y ; \sigma_{0}$ e $\varepsilon_{0}$ são vetores coluna formados com as componentes dos tensores de tensão e deformação iniciais, respectivamente; e $\boldsymbol{D}$ e $\boldsymbol{f}$ são as matrizes de rigidez e flexibilidade respectivamente, para materiais elásticos lineares isótropos.

- Equações de Equilíbrio:

As equações de equilíbrio passam a ter a seguinte apresentação num domínio bidimensional $\Omega$ : 


$$
\boldsymbol{L} \boldsymbol{\sigma}+\boldsymbol{b}=\mathbf{0}, \quad \text { em } \Omega
$$

onde:

$$
\boldsymbol{L}=\left[\begin{array}{ccc}
\frac{\partial}{\partial x} & 0 & \frac{\partial}{\partial y} \\
0 & \frac{\partial}{\partial y} & \frac{\partial}{\partial x}
\end{array}\right]
$$

é o operador diferencial divergente e $\boldsymbol{b}$ é o vetor de forças volúmicas.

- Equação de Compatibilidade:

A equação de compatibilidade é dada por:

$$
\varepsilon-\boldsymbol{L}^{T} \boldsymbol{u}=\mathbf{0}, \text { em } \Omega,
$$

onde:

$$
\boldsymbol{u}=\left\{\begin{array}{l}
\boldsymbol{u}_{\boldsymbol{x}} \\
\boldsymbol{u}_{\boldsymbol{y}}
\end{array}\right\}
$$

é o vetor de deslocamentos.

As condições de contorno da elasticidade plana são:

$$
\begin{aligned}
& \overline{\boldsymbol{u}}-\boldsymbol{u}=\mathbf{0}, \mathrm{em} \Gamma_{u} \\
& \overline{\boldsymbol{t}}-\boldsymbol{N} \sigma=\mathbf{0}, \text { em } \Gamma_{t}
\end{aligned}
$$

onde:

$$
N=\left[\begin{array}{ccc}
n_{x} & 0 & n_{y} \\
0 & n_{y} & n_{x}
\end{array}\right]
$$

é a matriz construída com as componentes do vetor $\boldsymbol{n}$ normal ao contorno, $\overline{\boldsymbol{u}}$ é o vetor dos deslocamentos impostos em $\Gamma_{u}$ e $\overline{\boldsymbol{t}}=\left[\begin{array}{l}\overline{\boldsymbol{t}}_{\boldsymbol{x}} \\ \overline{\boldsymbol{t}}_{\boldsymbol{y}}\end{array}\right]$ é o vetor das forças superficiais aplicadas em $\Gamma_{t}$. 


\subsection{Princípios Variacionais}

Os princípios variacionais têm grande importância na Mecânica servindo de base para o desenvolvimento de métodos numéricos. Por generalidade, pode-se dizer que um princípio variacional especifica uma quantidade escalar (funcional), definida por expressões integrais sobre o domínio $\Omega$ e contorno $\Gamma$ do sólido, do tipo:

$$
\Pi(u)=\int_{\Omega} R\left(u, \frac{\partial u}{\partial x}, \ldots\right) d \Omega+\int_{\Gamma} M\left(u, \frac{\partial u}{\partial x}, \ldots\right) d \Gamma
$$

onde $\boldsymbol{u}$ é uma função supostamente contínua e com derivadas contínuas e $\boldsymbol{R}$ e $\boldsymbol{M}$ são operadores diferenciais.

Em Mecânica dos Sólidos a condição de estacionariedade de $\Pi$ expressa a chamada forma fraca do PVC. Normalmente essa forma resulta da imposição da nulidade da primeira variação do funcional para qualquer variação compatível $\delta \boldsymbol{u}$ da função $\boldsymbol{u}$. Assim:

$$
\delta \Pi(u)=0
$$

Levando-se em conta a definição eq.(2.11), a condição dada pela eq.(2.12), pode ser escrita após realizar algumas diferenciações, na forma:

$$
\delta \Pi(u)=\int_{\Omega} \delta u^{T} A(u) d \Omega+\int_{\Gamma} \delta u^{T} B(u) d \Gamma=0
$$

Como a eq. (2.13) deve ser válida para qualquer variação de $\delta \boldsymbol{u}$, a sua nulidade fica garantida se:

$$
\begin{aligned}
& \boldsymbol{A}(\boldsymbol{u})=\mathbf{0} \text { em } \Omega \\
& \boldsymbol{B}(\boldsymbol{u})=\mathbf{0} \text { em } \Gamma
\end{aligned}
$$

Se $A(u)$ coincide exatamente com a equação diferencial que rege o PVC na forma forte e $\boldsymbol{B}(\boldsymbol{u})$ coincide com as relações referentes às condições de contorno, o princípio variacional será denominado natural. As expressões definidas na eq.(2.14) são conhecidas como equações diferenciais de Euler. 
Considere-se, agora, o problema de calcular a primeira variação do funcional $\Pi(u)$, eq.(2.11), obedecendo a um certo conjunto de restrições expresso por equações diferencias e representado na forma:

$$
\boldsymbol{C}(\boldsymbol{u})=\mathbf{0}, \mathrm{em} \Omega
$$

Esta restrição pode ser combinada com a eq. (2.11) mediante a técnica dos multiplicadores de Lagrange, formando, assim, outro funcional:

$$
\bar{\Pi}(u, \lambda)=\Pi(u)+\int_{\Omega} \lambda^{T} C(u) d \Omega
$$

onde $\lambda$ é um vetor de funções de coordenadas linearmente independentes no domínio $\Omega$ conhecidas como multiplicadores de Lagrange. A primeira variação desse novo funcional resulta:

$$
\bar{\delta} \bar{\Pi}(u, \lambda)=\delta \Pi(u)+\int_{\Omega} \lambda^{T} \delta C(u) d \Omega+\int_{\Omega} \delta \lambda^{T} C(u) d \Omega
$$

Uma condição para que a eq.(2.17) satisfaça a condição de estacionariedade expressa pela eq.(2.12), é a nulidade simultânea das integrais em $\Omega$.

De uma maneira geral, restrições podem ser introduzidas também em pontos do contorno. Por exemplo, se ao caso anterior for acrescentado que $\boldsymbol{u}$ deve obedecer também a:

$$
\boldsymbol{E}(\boldsymbol{u})=\boldsymbol{0} \text { em } \Gamma
$$

o funcional da eq. (2.16) receberá o termo: $\int_{\Gamma} \lambda^{T} \boldsymbol{E}(\boldsymbol{u}) d \Gamma$.

Embora os multiplicadores de Lagrange sejam introduzidos como uma técnica matemática necessária para que se cumpram certas restrições, observa-se que na maioria das situações físicas eles podem ser identificados com certas quantidades físicas de grande importância para o modelo original. Na vasta literatura sobre cálculo das variações aparece com freqüência esta identificação, como nos exemplos dados por Washizu (1975). 


\subsection{Princípios Variacionais Mistos}

No item anterior foi apresentada a caracterização matemática geral dos Princípios Variacionais. Dentre os Princípios Variacionais clássicos, citam-se: o Princípio dos Trabalhos Virtuais (PTV), Princípio da Mínima Energia Potencial, Princípio da Mínima Energia Potencial Complementar. O Princípio dos Trabalhos Virtuais (PTV) tem uma vasta abordagem para muitos problemas dentro do campo da mecânica estrutural. Ele é considerado um princípio geral, pois é aplicado em análises que englobam tanto comportamento elástico, quanto anelástico, carregamento tanto mecânico quanto térmico, e em problemas envolvendo estabilidade estrutural e dinâmica estrutural.

Tanto o Princípio dos Trabalhos Virtuais (PTV) como os demais citados tem uma característica em comum: todos estão definidos sobre campos únicos, sejam eles de tensão ou de deslocamento. Nesta etapa serão exibidas duas outras formulações variacionais que, diferentemente das anteriores, envolvem funcionais dependentes de dois e três campos. Na literatura, estas duas formulações são conhecidas como funcional de Hellinger-Reissner (funcional de dois campos) e funcional de Hu-Washizu ou Princípio Variacional Generalizado (funcional de três campos).

Ao se definir o clássico funcional da energia potencial $(\Pi(\varepsilon, \boldsymbol{u})=\boldsymbol{U}(\varepsilon)+\boldsymbol{V}(\boldsymbol{u})$, aplicado normalmente à análise de estruturas elásticas), admite-se que o campo das deformações se relaciona com o campo dos deslocamentos, como mostra a eq.(2.8).

Ainda, no contorno $\Gamma_{u}$, os deslocamentos prescritos são expressos na forma da eq.(2.9).

As condições representadas pelas eq.(2.8) e (2.9) podem ser entendidas como restrições e incluídas num novo funcional:

$$
\bar{\Pi}=\Pi(\varepsilon, u)-\int_{\Omega} \lambda_{1}^{T}\left(\varepsilon-L^{T} u\right) d \Omega-\int_{\Gamma_{u}} \lambda_{2}^{T}(u-\bar{u}) d \Gamma
$$

onde se introduziram dois multiplicadores de Lagrange independentes, $\lambda_{1}$ definido no domínio $\Omega$ e $\lambda_{2}$ definido somente em $\Gamma_{u}$.

Efetuando-se a primeira variação do funcional expresso pela eq. (2.19), tem-se:

$$
\delta \bar{\Pi} \equiv \mathbf{0}=\delta \Pi(\varepsilon, u)-\int_{\Omega} \delta \lambda_{1}^{T}\left(\varepsilon-L^{T} u\right) d \Omega-\int_{\Gamma_{u}} \delta \lambda_{2}^{T}(u-\bar{u}) d \Gamma-\int_{\Omega} \lambda_{1}^{T}\left(\delta \varepsilon-L^{T} \delta u\right) d \Omega-\int_{\Gamma_{u}} \lambda_{2}^{T} \delta u d \Gamma
$$

Usando o teorema de Green, pode-se escrever: 


$$
\int_{\Omega} \lambda_{1}^{T} L^{T} \delta u d \Omega=-\int_{\Omega} \delta u^{T} L \lambda_{1} d \Omega+\int_{\Gamma} \delta u^{T} N \lambda_{1} d \Gamma
$$

onde $\boldsymbol{N}$ é um operador que reúne as componentes da normal ao contorno . Assim, levando-se em conta que $\delta \Pi(\varepsilon, u)=\int_{\Omega} \delta \varepsilon^{T} \sigma(\varepsilon) d \Omega-\int_{\Omega} \delta u^{T} b d \Omega-\int_{\Gamma} \delta u^{T} \bar{t} d \Gamma$, a eq.(2.20) resulta:

$$
\begin{aligned}
& \int_{\Omega} \delta \varepsilon^{T}\left(\sigma(\varepsilon)-\lambda_{1}\right) d \Omega-\int_{\Omega} \delta u^{T}\left(L \lambda_{1}+b\right) d \Omega+\int_{\Gamma_{t}} \delta u^{T}\left(N \lambda_{1}-\bar{t}\right) d \Gamma+ \\
& +\int_{\Gamma_{u}} \delta u^{T}\left(N \lambda_{1}-\lambda_{2}\right) d \Gamma-\int_{\Omega} \delta \lambda_{1}^{T}\left(\varepsilon-L^{T} u\right) d \Omega-\int_{\Gamma_{u}} \delta \lambda_{2}^{T}(u-\bar{u}) d \Gamma \equiv 0
\end{aligned}
$$

Analisando-se as parcelas de variações de energia envolvidas na eq.(2.22), notase que $\lambda_{1}$ e $\lambda_{2}$ multiplicam termos em deformação e deslocamento, respectivamente. Assim sendo, os multiplicadores de Lagrange podem ser identificados como campos de tensão e de força por unidade de superfície:

$$
\lambda_{1}=\sigma \text { e } \lambda_{2}=t
$$

Com esta identificação, a eq.(2.19) recebe o nome de princípio de Hu-Washizu e passa a ser enunciada como o seguinte funcional:

$$
\bar{\Pi}=\Pi(\varepsilon, u)-\int_{\Omega} \sigma^{T}\left(\varepsilon-L^{T} u\right) d \Omega-\int_{\Gamma_{u}} t^{T}(u-\bar{u}) d \Gamma
$$

Este funcional envolve de modo independente os campos $\boldsymbol{\varepsilon}, \boldsymbol{u}$ e $\boldsymbol{\sigma}$ no domínio $\Omega$ e $\boldsymbol{t}$ no contorno $\Gamma$.

Por outro lado, a soma das energias de deformação e energia de deformação complementar pode ser escrita como:

$$
U(\varepsilon)+U(\sigma)^{*}=\int_{\Omega} \sigma^{T} \varepsilon d \Omega
$$

Fazendo-se a variação da eq. (2.25), tem-se:

$$
\delta U+\delta U^{*}=\int_{\Omega} \delta \varepsilon^{T} \sigma d \Omega+\int_{\Omega} \delta \sigma^{T} \varepsilon d \Omega
$$


Pode-se formular outra expressão variacional substituindo-se $\boldsymbol{U}(\varepsilon)$ da eq.(2.25) na expressão da energia potencial total $\Pi(\varepsilon, \boldsymbol{u})=\boldsymbol{U}(\varepsilon)+\boldsymbol{V}(\boldsymbol{u})$, assim:

$$
\Pi(\varepsilon, u)=\int_{\Omega} \sigma^{T} \varepsilon d \Omega-U(\sigma)^{*}+V(u)
$$

A eq. (2.24) toma então a seguinte forma:

$$
\bar{\Pi}=\int_{\Omega} \sigma^{T} \varepsilon d \Omega-U(\sigma)^{*}-\int_{\Omega} u^{T} b d \Omega-\int_{\Gamma_{t}} u^{T} \bar{t} d \Gamma-\int_{\Omega} \sigma^{T}\left(\varepsilon-L^{T} u\right) d \Omega-\int_{\Gamma_{u}} t^{T}(u-\bar{u}) d \Gamma
$$

Admitindo-se que $\varepsilon$ não é mais independente e que satisfaz as condições de compatibilidade, pode-se escrever a eq. (2.28) como uma nova expressão variacional conhecida como princípio variacional de Reissner-Helinger. Assim:

$$
\bar{\Pi}=\int_{\Omega} \sigma^{T} \varepsilon d \Omega-U^{*}(\sigma)-\int_{\Omega} u^{T} b d \Omega-\int_{\Gamma_{t}} u^{T} \bar{t} d \Gamma
$$

$\operatorname{com} \varepsilon=\boldsymbol{L}^{T} \boldsymbol{u}$ em $\Omega ; \boldsymbol{u}-\overline{\boldsymbol{u}}=\mathbf{0}$ em $\Gamma_{\boldsymbol{u}}$. Neste caso as únicas variáveis independentes para este novo funcional são os campos $\sigma$ e $\boldsymbol{u}$ em $\Omega$ e $\boldsymbol{u}$ em $\Gamma_{t}$.

\subsection{O MEF na Mecânica dos Sólidos}

O objetivo desse item é contextualizar as FHMT, FHT e FHTFT propostas no trabalho de Freitas, Almeida e Pereira (1996), dentro da classificação do MEF para a mecânica dos sólidos fornecida em Desai e Abel (1972) e Pian e Tong (1969).

Já foi enfatizado que o MEF pode apresentar diferentes características na aproximação de um PVC, dependendo do princípio variacional e dos espaços de funções adotadas. Quando são mudados o princípio variacional e os respectivos espaços, uma diferente formulação em elementos finitos é aplicada para aproximação do PVC.

No item anterior, destacou-se que o problema elástico linear pode ser expresso em forma fraca, valendo-se de diversos princípios variacionais. Vale salientar que um princípio variacional associado não é necessário para o desenvolvimento de uma formulação do MEF.

Na tabela a seguir, formulada com base em Desai e Abel (1972) e Pian e Tong (1969), apresentam-se os vários métodos do MEF na mecânica dos sólidos relacionados 
com seus respectivos princípios variacionais e as variáveis a serem determinadas em cada um desses métodos.

Tabela 2.1 - Classificação do Método dos Elementos Finitos na Mecânica dos Sólidos.

\begin{tabular}{|c|c|c|c|c|}
\hline Formulação & $\begin{array}{c}\text { Princípio } \\
\text { Variacional }\end{array}$ & $\begin{array}{c}\text { O Que é } \\
\text { Aproximado no } \\
\text { Domínio de Cada } \\
\text { Elemento? }\end{array}$ & $\begin{array}{c}\text { O Que é } \\
\text { Aproximado no } \\
\text { Contorno de } \\
\text { Cada Elemento? }\end{array}$ & $\begin{array}{l}\text { Parâmetros a } \\
\text { Serem } \\
\text { Determinados }\end{array}$ \\
\hline Deslocamento & $\begin{array}{l}\text { Mínima Energia } \\
\text { Potencial }\end{array}$ & $\begin{array}{l}\text { Distribuição suave dos } \\
\text { deslocamentos }\end{array}$ & $\begin{array}{c}\text { Continuidade nos } \\
\text { deslocamentos }\end{array}$ & $\begin{array}{c}\text { Deslocamentos nodais } \\
\text { no domínio }\end{array}$ \\
\hline Equilíbrio & $\begin{array}{l}\text { Mínima Energia } \\
\text { Complementar }\end{array}$ & $\begin{array}{c}\text { Distribuição suave e } \\
\text { equilibrada das } \\
\text { tensões } \\
\end{array}$ & $\begin{array}{c}\text { Equilíbrio das forças } \\
\text { nos contorno }\end{array}$ & $\begin{array}{l}\text { Tensões nodais no } \\
\text { domínio }\end{array}$ \\
\hline $\begin{array}{l}\text { Híbrida-Tensão } \\
\text { (FHT) }\end{array}$ & $\begin{array}{c}\text { Mínima } \\
\text { Energia } \\
\text { Complementar } \\
\text { Modificado }\end{array}$ & $\begin{array}{c}\text { Distribuição suave e } \\
\text { equilibrada das } \\
\text { tensões }\end{array}$ & $\begin{array}{l}\text { Deslocamentos } \\
\text { compatíveis }\end{array}$ & $\begin{array}{c}\text { Deslocamentos } \\
\text { nodais no contorno } \\
\text { e campos de tensão } \\
\text { equilibrados no } \\
\text { domínio }\end{array}$ \\
\hline Mista (2Campos) & Reissner & $\begin{array}{c}\text { Distribuição suave } \\
\text { das tensões e } \\
\text { deslocamentos }\end{array}$ & $\begin{array}{c}\text { Continuidade nas } \\
\text { tensões e } \\
\text { deslocamentos }\end{array}$ & $\begin{array}{l}\text { Deslocamentos e } \\
\text { tensões nodais no } \\
\text { domínio }\end{array}$ \\
\hline Mista (3 Campos) & Wu-Whashizu & $\begin{array}{l}\text { Distribuição suave } \\
\text { das tensões, } \\
\text { deslocamentos e } \\
\text { deformações }\end{array}$ & $\begin{array}{l}\text { Distribuição de } \\
\text { forças no contorno }\end{array}$ & $\begin{array}{c}\text { Deslocamentos, } \\
\text { tensões, } \\
\text { deformações nodais } \\
\text { no domínio e forças } \\
\text { no contorno }\end{array}$ \\
\hline $\begin{array}{c}\text { Híbrida - Mista Tensão } \\
\text { (FHMT) }\end{array}$ & Reissner* & $\begin{array}{c}\text { Distribuição suave } \\
\text { das tensões e } \\
\text { deslocamentos }\end{array}$ & $\begin{array}{l}\text { Deslocamentos } \\
\text { compatíveis }\end{array}$ & $\begin{array}{c}\text { Tensões e } \\
\text { deslocamentos } \\
\text { nodais no domínio e } \\
\text { deslocamentos } \\
\text { nodais no contorno }\end{array}$ \\
\hline $\begin{array}{c}\text { Híbrida - Trefftz } \\
\text { Tensão } \\
\text { (FHTFT) }\end{array}$ & $\begin{array}{c}\text { Mínima } \\
\text { Energia } \\
\text { Complementar } \\
\text { Modificado }\end{array}$ & $\begin{array}{c}\text { As tensões devem } \\
\text { satisfazer a restrição } \\
\text { de Trefftz }\end{array}$ & $\begin{array}{l}\text { Deslocamentos } \\
\text { compatíveis }\end{array}$ & $\begin{array}{c}\text { Deslocamentos } \\
\text { nodais no contorno } \\
\text { e campos de tensão } \\
\text { que satisfazem a } \\
\text { restrição de Trefftz } \\
\text { no domínio }\end{array}$ \\
\hline
\end{tabular}

*O funcional de Reissner é modificado para uma forma híbrida-mista.

** A restrição de Trefftz implica que as aproximações no domínio do elemento satisfaçam o sistema de equações diferenciais que governa o PVC. No caso de problemas da elasticidade linear, assume-se que as aproximações do campo de tensões são derivadas de potencias de tensões bi-harmônicos que satisfaçam a equação de Beltrami. 


\section{Formulação Híbrida-Mista de Tensão para Elasticidade}

\subsection{Considerações Iniciais}

No capítulo anterior, foram evidenciados alguns princípios variacionais para a Elasticidade, restritas aos regimes de pequenas deformações e deslocamentos. Observase que estes proporcionam uma abordagem natural para determinação de soluções aproximadas de problemas elastoestáticos e o MEF proporciona uma técnica sistemática para geração de soluções aproximativas.

Dependendo do princípio variacional adotado na análise, pode-se aproximar um ou mais dos seguintes campos: tensão, deformação e deslocamento. Quanto menor o número de campos a aproximar, mais condições de restrições devem ser obedecidas "a priori” pela aproximação. Especificamente, na formulação clássica em deslocamentos, com aplicação do MEF, deve-se garantir que os elementos finitos da discretização sejam compatíveis, ou seja, neles sejam satisfeitas a condição de compatibilidade eq.(2.4) e a condição de contorno de Dirichlet eq.(2.9).

Para os princípios variacionais mistos, podem ser aproximadas duas variáveis (funcional de Reissner-Helinger eq.(2.29)) ou três variáveis (funcional de Hu-Washizu eq.(2.28)). Nestes casos os campos de tensão e deslocamento são incompatíveis, isto é, não satisfazem "a priori" a lei constitutiva eq.(2.1) mesmo que seja satisfeita a equação de compatibilidade eq.(2.4).

Apresentar-se-á no que segue a formulação adotada neste trabalho, onde, para o modelo Híbrido-Misto de Tensão, além das aproximações independentes dos campos de tensão e deslocamento no domínio da análise, será aproximado de forma independente o campo de deslocamento no contorno do problema.

Assume-se também para o desenvolvimento da FHMT as seguintes condições: as deformações $\left(\varepsilon_{0}\right)$ residuais, tensões $\left(\sigma_{0}\right)$ residuais, forças de volume $(\boldsymbol{b}) \mathrm{e}$ 
deslocamentos $\overline{\boldsymbol{u}}$ prescritos no contorno serão considerados nulos. Além disso, como já comentado, serão adotadas as hipóteses de linearidade geométrica e física.

\subsection{Formulação Geral Híbrida-Mista de Tensão}

Seja o funcional de Reissner-Helinger dado pela eq.(2.29) escrita da seguinte forma:

$$
\Pi(u, \sigma)=\int_{\Omega} \sigma^{T} \varepsilon d \Omega-\int_{\Omega} \frac{1}{2} \sigma^{T} f \sigma d \Omega-\int_{\Omega} u^{T} b d \Omega-\int_{\Gamma_{t}} u^{T} \bar{t} d \Gamma
$$

com $\boldsymbol{\varepsilon}=\boldsymbol{L}^{\boldsymbol{T}} \boldsymbol{u}$ em $\Omega ; \boldsymbol{u}-\overline{\boldsymbol{u}}=\mathbf{0}$ em $\Gamma_{\boldsymbol{u}}$ e sendo $\boldsymbol{f}$ a matriz de flexibilidade definida na lei constitutiva da eq.(2.6).

Podemos substituir a equação de compatibilidade, eq.(2.8), na primeira parcela da eq.(3.1). Assim:

$$
\Pi(u, \sigma)=\int_{\Omega} \sigma^{T} L^{T} u d \Omega-\int_{\Omega} \frac{1}{2} \sigma^{T} f \sigma d \Omega-\int_{\Omega} u^{T} b d \Omega-\int_{\Gamma_{t}} u^{T} \bar{t} d \Gamma
$$

Aplicando o teorema de Green a primeira integral da eq.(3.2), tem-se:

$$
\int_{\Omega} \sigma^{T} L^{T} u d \Omega=-\int_{\Omega} u^{T} L \sigma d \Omega+\int_{\Gamma_{t}} u^{T}(N \sigma) d \Gamma+\int_{\Gamma_{u}} \bar{u}^{T}(N \sigma) d \Gamma
$$

Nessas condições, a eq.(3.2) assume a forma:

$$
\Pi\left(u, \sigma, u_{\Gamma}\right)=-\int_{\Omega} \frac{1}{2} \sigma^{T} f \sigma d \Omega-\int_{\Omega} u^{T}(L \sigma+b) d \Omega+\int_{\Gamma_{t}} u_{\Gamma}^{T}(N \sigma-\bar{t}) d \Gamma+\int_{\Gamma_{u}} \bar{u}^{T}(N \sigma) d \Gamma
$$

A eq.(3.4) é forma híbrida-mista da eq.(3.2), pois agora além da incompatibilidade entre os campos de tensão $(\sigma)$ e deslocamento $(\boldsymbol{u})$ no domínio $(\Omega)$, temos um campo de deslocamento definido também na parte contorno $\left(\Gamma_{t}\right)$ onde as forças de superfície estão definidas. Assim o funcional da eq.(3.4) possui três variáveis independentes: tensão $(\sigma)$ e deslocamento $(\boldsymbol{u})$ no domínio $(\Omega)$ e o deslocamento no contorno $\left(\boldsymbol{u}_{\Gamma}\right)$.

A condição de estacionariedade do funcional dado pela eq.(3.4) é:

$$
\delta \Pi=\boldsymbol{0} \Rightarrow \frac{\partial \Pi}{\partial \sigma} \cdot d \sigma+\frac{\partial \Pi}{\partial u} \cdot d u+\frac{\partial \Pi}{\partial u_{\Gamma}} \cdot d u_{\Gamma}=0
$$


Para nulidade da eq.(3.5), considerando-se qualquer variação dos campos de tensão $(\sigma)$ e deslocamento $(\boldsymbol{u})$ no domínio $(\Omega)$ e do deslocamento no contorno $\left(\boldsymbol{u}_{\Gamma}\right)$, deve-se ter:

$$
\begin{aligned}
& \frac{\partial \Pi}{\partial \sigma}=0 \\
& \frac{\partial \Pi}{\partial \boldsymbol{u}}=\mathbf{0} \\
& \frac{\partial \Pi}{\partial \boldsymbol{u}_{\Gamma}}=\mathbf{0}
\end{aligned}
$$

Desenvolvendo as derivadas das eq.(3.6), eq.(3.7) e eq.(3.8), tem-se:

$$
\begin{aligned}
& \frac{\partial \Pi}{\partial \sigma}=\mathbf{0} \Rightarrow \int_{\Omega} \delta \sigma^{T} f \sigma d \Omega+\int_{\Omega} \boldsymbol{u}^{T}(L \delta \sigma) d \Omega-\int_{\Gamma_{t}}{u_{\Gamma}^{T}}^{T}(N \delta \sigma) d \Gamma-\int_{\Gamma_{u}} \overline{\boldsymbol{u}}^{T}(N \delta \sigma) d \Gamma=\mathbf{0} \\
& \frac{\partial \Pi}{\partial \boldsymbol{u}}=\mathbf{0} \Rightarrow \int_{\Omega} \delta \boldsymbol{u}^{T}(L \sigma+b) d \Omega=0 \\
& \frac{\partial \Pi}{\partial u_{\Gamma}}=\mathbf{0} \Rightarrow \int_{\Gamma_{t}} \delta u_{\Gamma}^{T}(N \sigma-\overline{\boldsymbol{t}}) d \Gamma=\mathbf{0}
\end{aligned}
$$

Vale ressaltar que se pode chegar às equações integrais dadas pelas eq.(3.9), eq.(3.10) e eq.(3.11) por outra abordagem, realizando-se ponderações de Galerkin, ou forma fraca, das eq.(2.7), eq.(2.8) e eq.(2.10). Nesse caso, resultam:

$$
\begin{aligned}
& \int_{\Omega} \delta u^{T}(\boldsymbol{L} \sigma+b) d \Omega=0 \\
& \int_{\Omega} \delta \sigma^{T}\left(\varepsilon-L^{T} u\right) d \Omega=0 \\
& \int_{\Gamma_{T}} \delta u_{\Gamma}^{T}(N \sigma-\bar{t}) d \Gamma=\mathbf{0}
\end{aligned}
$$

Da eq.(3.13), com ajuda da eq.(2.6), eq.(2.9) e do Teorema da Divergência, vem:

$$
\int_{\Omega} \delta \sigma^{T}\left(f \sigma+L^{T} u\right) d \Omega-\int_{\Gamma_{t}}(N \delta \sigma)^{T} u_{\Gamma} d \Gamma-\int_{\Gamma u}(N \delta \sigma)^{T} \bar{u} d \Gamma=0
$$


Assim as eq.(3.9), eq.(3.10) e eq.(3.11) são exatamente iguais as eq.(3.15), eq.(3.13) e eq.(3.14), respectivamente.

\subsection{Forma Aproximada do Modelo Híbrido-Misto de Tensão}

\subsubsection{Aproximação do Campo de Tensões no Domínio}

Na FHMT, o campo de tensão no domínio $(\Omega)$ é uma variável independente, podendo assim, ser também aproximado por um conjunto de funções interpoladoras. Denominando-se $\boldsymbol{S}_{\Omega}$ a matriz com as funções de interpolação e $\boldsymbol{s}_{\Omega}$ o vetor que agrupa os graus de liberdade do campo de tensão, então:

$$
\hat{\sigma}=S_{\Omega} s_{\Omega}
$$

\subsubsection{Aproximação do Campo de Deslocamentos no Domínio}

O campo de deslocamentos no domínio $(\Omega)$ tem sua representação aproximada dada na seguinte forma:

$$
\hat{\boldsymbol{u}}=\boldsymbol{U}_{\Omega} \boldsymbol{q}_{\Omega}
$$

onde $U_{\Omega}$ é a matriz que guarda as funções de aproximação do campo de deslocamento no domínio $(\Omega)$ e $\boldsymbol{q}_{\Omega}$ é o vetor com os graus de liberdade.

\subsubsection{Aproximação do Campo de Deslocamentos no Contorno}

O campo de deslocamentos aproximados no contorno $(\Gamma)$ tem a seguinte representação:

$$
\hat{\boldsymbol{u}}_{\Gamma}=\boldsymbol{U}_{\Gamma} \boldsymbol{q}_{\Gamma}
$$

onde $\boldsymbol{U}_{\Gamma}$ é a matriz que coleta as funções de aproximação do campo de deslocamento no contorno $(\Gamma)$ e $q_{\Gamma}$ é o vetor que guarda os graus de liberdade.

\subsubsection{Aproximação Geral do Modelo Híbrido-Misto}

Os campos virtuais de tensão, deslocamento e deslocamento no contorno podem ser representados pelo mesmo conjunto de funções de aproximação apresentados anteriormente. 
Assim, sejam as eq.(3.9), eq.(3.10) e eq.(3.11) escritas com a consideração das aproximações adotadas:

$$
\begin{aligned}
& \delta_{\Omega}^{T}\left(\int_{\Omega} S_{\Omega}^{T} f S_{\Omega} d \Omega\right) s_{\Omega}+\delta \delta_{\Omega}^{T}\left(\int_{\Omega}\left(L S_{\Omega}\right)^{T} U_{\Omega} d \Omega\right) q_{\Omega}-\delta \delta_{\Omega}^{T}\left(\int_{\Gamma_{t}}\left(N S_{\Omega}\right)^{T} U_{\Gamma} d \Gamma\right) q_{\Gamma}-\delta \delta_{\Omega}^{T}\left(\int_{\Gamma_{u}}\left(N S_{\Omega}\right)^{T} \bar{u} d \Gamma\right)=\mathbf{0} \\
& \delta q_{\Omega}\left(\int_{\Omega} U_{\Omega}^{T}\left(L S_{\Omega}+b\right) d \Omega\right)=\mathbf{0} \\
& \delta q_{\Gamma}^{T}\left(\int_{\Gamma_{t}} U_{\Gamma}^{T}\left(N S_{\Omega}-\bar{t}\right) d \Gamma\right) \boldsymbol{s}_{\Omega}=\mathbf{0}
\end{aligned}
$$

Como as variações $\delta s_{\Omega}, \delta q_{\Omega}$ e $\delta q_{\Gamma}$ são quaisquer, para garantir a nulidade das eq.(3.19), eq.(3.20) e eq.(3.21), deve-se ter:

$$
\begin{aligned}
& \int_{\Omega} S_{\Omega}^{T} f S_{\Omega} d \Omega s_{\Omega}+\int_{\Omega}\left(L S_{\Omega}\right)^{T} U_{\Omega} d \Omega q_{\Omega}-\int_{\Gamma_{t}}\left(N S_{\Omega}\right)^{T} U_{\Gamma} d \Gamma q_{\Gamma}=\int_{\Gamma_{u}}\left(N S_{\Omega}\right)^{T} \bar{u} d \Gamma \\
& \int_{\Omega} U_{\Omega}^{T}\left(L S_{\Omega}\right) d \Omega s_{\Omega}=-\int_{\Omega} U_{\Omega}^{T} b d \Omega \\
& -\int_{\Gamma_{t}} U_{\Gamma}^{T}\left(N S_{\Omega}\right) d \Gamma s_{\Omega}=-\int_{\Gamma_{t}} U_{\Gamma}^{T}(\bar{t}) d \Gamma
\end{aligned}
$$

As eq. (3.22), eq.(3.23) e eq.(3.24) levam ao seguinte sistema de equações lineares:

$$
\left[\begin{array}{ccc}
\boldsymbol{F} & \boldsymbol{A}_{\Omega} & -\boldsymbol{A}_{\Gamma} \\
\boldsymbol{A}_{\Omega}^{T} & \mathbf{0} & \mathbf{0} \\
-\boldsymbol{A}_{\Gamma}^{T} & \mathbf{0} & \mathbf{0}
\end{array}\right]\left[\begin{array}{c}
\boldsymbol{s}_{\Omega} \\
\boldsymbol{q}_{\Omega} \\
\boldsymbol{q}_{\Gamma}
\end{array}\right]=\left[\begin{array}{c}
\boldsymbol{e}_{\Gamma} \\
-\boldsymbol{Q}_{\Omega} \\
-\boldsymbol{Q}_{\Gamma}
\end{array}\right]
$$

onde foram introduzidas as seguintes matrizes:

$$
\begin{aligned}
& F=\int_{\Omega} S_{\Omega}^{T} f S_{\Omega} d \Omega \\
& A_{\Omega}=\int_{\Omega}\left(L S_{\Omega}\right)^{T} U_{\Omega} d \Omega \\
& A_{\Gamma}=\int_{\Gamma_{t}}\left(N S_{\Omega}\right)^{T} U_{\Gamma} d \Gamma
\end{aligned}
$$




$$
\begin{aligned}
& e_{\Gamma}=\int_{\Gamma_{u}}\left(N S_{\Omega}\right)^{T} \bar{u} d \Gamma \\
& Q_{\Omega}=\int_{\Omega} U_{\Omega}^{T} b d \Omega \\
& Q_{\Gamma}=\int_{\Gamma_{t}} U_{\Gamma}^{T}(\bar{t}) d \Gamma
\end{aligned}
$$

Para a situação em que as forças de volume $(\boldsymbol{b})$ são desconsideradas e o vetor de deslocamentos $\overline{\boldsymbol{u}}$ é prescrito como nulo no contorno, então $\boldsymbol{e}_{\Gamma}=\boldsymbol{Q}_{\Omega}=\mathbf{0}$ e o sistema de equações, eq.(3.25), toma a seguinte forma:

$$
\left[\begin{array}{ccc}
\boldsymbol{F} & \boldsymbol{A}_{\Omega} & -\boldsymbol{A}_{\Gamma} \\
\boldsymbol{A}_{\Omega}^{T} & \mathbf{0} & \mathbf{0} \\
-\boldsymbol{A}_{\Gamma}^{T} & \mathbf{0} & \mathbf{0}
\end{array}\right]\left[\begin{array}{c}
\boldsymbol{s}_{\Omega} \\
\boldsymbol{q}_{\Omega} \\
\boldsymbol{q}_{\Gamma}
\end{array}\right]=\left[\begin{array}{c}
\mathbf{0} \\
\mathbf{0} \\
-\boldsymbol{Q}_{\Gamma}
\end{array}\right]
$$

Observa-se que a matriz $\boldsymbol{F}$ é uma matriz quadrada que depende das constantes elásticas do material. Além disso, sua ordem é definida pela ordem da matriz que guarda as interpolações do campo de tensão. A dimensão da matriz $A_{\Omega}$ é função respectivamente das ordens das matrizes que coletam as aproximações dos campos de tensão e deslocamento no domínio. Já a dimensão da matriz $\boldsymbol{A}_{\Gamma}$ depende também da matriz que guarda as aproximações do campo de tensão e da matriz que coleta as interpolações do campo de deslocamento no contorno.

Para que a eq.(3.32) seja válida é necessário que as funções de aproximação adotadas tenham continuidade suficiente para que as integrais que levam às matrizes $\boldsymbol{F}$, $\boldsymbol{A}_{\Omega}$ e $\boldsymbol{A}_{\Gamma}$ e ao vetor $\boldsymbol{Q}_{\Gamma}$ possam ser calculadas.

Serão discutidos posteriormente os aspectos numéricos para solução do sistema de equações lineares da eq.(3.32). 


\section{Formulação Geral Híbrida-Mista com Malha de Cobertura}

\subsection{Introdução}

Neste capítulo serão abordadas as características gerais da FHMT com a utilização de uma malha de cobertura. Após esta abordagem geral, particularizar-se-á a análise para uma malha de elementos finitos isoparamétricos quadrangulares convencionais de quatro nós. Estes serão os elementos usados na análise numérica com o Modelo Híbrido-Misto de Tensão.

\subsection{Modelo Híbrido Misto com Malha de Cobertura}

Seja o domínio $\Omega$ coberto por uma malha de $\boldsymbol{n}_{\Omega}$ elementos finitos. Esta malha é aqui denominada malha de cobertura. Sejam ainda $\Omega_{e}, \boldsymbol{e}=\mathbf{1}, \ldots \boldsymbol{n}_{\Omega}$, os domínios desses elementos finitos e $\Gamma_{i}, \boldsymbol{i}=\mathbf{1}, \ldots \boldsymbol{n}_{\Gamma}$, os lados dos elementos que pertencem ao contorno $\Gamma$ do domínio $\Omega$. Agora, sejam $\boldsymbol{s}_{\Omega_{e}}, \boldsymbol{q}_{\Omega_{e}}$ e $\boldsymbol{q}_{\Gamma_{i}}$ os vetores que guardam os graus de liberdade nodais correspondentes às tensões e deslocamentos pertencentes ao elemento $\boldsymbol{e}$ ou ao lado $\boldsymbol{i}$, respectivamente.

Os graus de liberdade nodais do elemento $\boldsymbol{e}$ podem ser colocados em correspondência com os graus de liberdade globais $\boldsymbol{s}_{\Omega}, \boldsymbol{q}_{\Omega}$ e $\boldsymbol{q}_{\Gamma}$ pelas seguintes relações:

$$
\begin{aligned}
& s_{\Omega_{e}}=\boldsymbol{F}_{\mathbf{e}} s_{\Omega}, e=1, \ldots n_{\Omega} \\
& q_{\Omega_{e}}=\boldsymbol{A}_{\Omega_{e}} q_{\Omega}, e=1, \ldots n_{\Omega} \\
& \boldsymbol{q}_{\Gamma_{i}}=\boldsymbol{A}_{\boldsymbol{r}_{i}} \boldsymbol{q}_{\Gamma}, i=1, \ldots n_{\Gamma}
\end{aligned}
$$


onde $\boldsymbol{F}_{\mathbf{e}}$ e $\boldsymbol{A}_{\boldsymbol{\Omega}_{\mathbf{e}}}$ são as matrizes Booleanas que extraem os graus de liberdade do elemento finito de domínio $\Omega_{e}$ e $\boldsymbol{A}_{\boldsymbol{r}}$ é a matriz Booleana que extrai os graus de liberdade do lado $\Gamma_{i}$ no contorno.

Considere-se que as aproximações do campo de tensões, eq.(3.16), e dos deslocamentos no domínio, eq.(3.17), sejam agora restritas do domínio $\Omega_{e}$ e dadas por:

$$
\begin{aligned}
& \hat{\sigma}_{e}=\boldsymbol{S}_{\Omega_{e}} \boldsymbol{s}_{\Omega_{e}} \\
& \hat{\boldsymbol{u}}_{e}=\boldsymbol{U}_{\Omega_{e}} \boldsymbol{q}_{\Omega_{e}}
\end{aligned}
$$

Da mesma forma a interpolação do campo de deslocamento no contorno, eq.(3.18), para um lado $\Gamma_{i}$ é dada por:

$$
\hat{\boldsymbol{u}}_{\Gamma_{i}}=\boldsymbol{U}_{\Gamma_{i}} \boldsymbol{q}_{\Gamma_{i}}
$$

$\boldsymbol{S}_{\Omega_{e}}, \boldsymbol{U}_{\Omega_{e}}$ e $\boldsymbol{U}_{\Gamma_{i}}$ são as matrizes que coletam respectivamente as funções de interpolação dos campos de tensão e deslocamento no domínio e deslocamento no contorno. A partir da contribuição de cada um dos elementos e lados que pertencem ao contorno as matrizes das eq. (3.26), eq.(3.27), eq.(3.28) e eq.(3.31) podem ser calculadas por meio de

$$
\begin{aligned}
& \boldsymbol{F}=\sum_{e=1}^{n_{\Omega}} \boldsymbol{F}_{\mathbf{e}}^{\boldsymbol{T}} \boldsymbol{F}_{e} \boldsymbol{F}_{\mathbf{e}} \\
& \boldsymbol{A}_{\Omega}=\sum_{e=1}^{n_{\Omega}} \boldsymbol{F}_{\mathbf{e}}^{\boldsymbol{T}} A_{\Omega_{e}} \boldsymbol{A}_{\Omega_{\mathbf{e}}} \\
& \boldsymbol{A}_{\Gamma}=\sum_{i=1}^{n_{\Gamma}} \boldsymbol{F}_{\mathbf{e}}^{\boldsymbol{T}} A_{\Gamma_{e}} \boldsymbol{A}_{\boldsymbol{r}_{\boldsymbol{i}}} \\
& \boldsymbol{Q}_{\Gamma}=\sum_{i=1}^{n_{\Gamma}} \boldsymbol{A}_{\boldsymbol{r}_{i}}^{\boldsymbol{T}} \boldsymbol{Q}_{\Gamma_{i}}
\end{aligned}
$$

onde:

$$
F_{e}=\int_{\Omega} S_{\Omega_{e}}^{T} f S_{\Omega_{e}} d \Omega
$$




$$
\begin{aligned}
& A_{\Omega_{e}}=\int_{\Omega}\left(L S_{\Omega_{e}}\right)^{T} U_{\Omega_{e}} d \Omega \\
& A_{\Gamma_{i}}=\int_{\Gamma_{t_{i}}}\left(N S_{\Omega_{e}}\right)^{T} U_{\Gamma_{i}} d \Gamma \\
& Q_{\Gamma_{i}}=\int_{\Gamma_{\Gamma_{i} i}} U_{\Gamma_{i}}^{T}(\bar{t}) d \Gamma
\end{aligned}
$$

As matrizes que reúnem as funções de interpolação para os campos de tensões e deslocamentos são formadas por sub-matrizes cujo número de nós está associado ao número de nós dos elementos. A ordem dessas sub-matrizes está relacionada ao número de graus de liberdade definido em cada nó. A representação aqui adotada para as matrizes é a seguinte:

$$
\begin{aligned}
& \boldsymbol{S}_{\Omega_{e}}=\left[\begin{array}{llll}
\sum_{1} & \sum_{\alpha} & \ldots & \sum_{N_{S}}
\end{array}\right] \\
& \boldsymbol{U}_{\Omega_{e}}=\left[\begin{array}{llll}
H_{1} & \boldsymbol{H}_{\delta} & \ldots & \boldsymbol{H}_{N_{U}}
\end{array}\right] \\
& \boldsymbol{U}_{\Gamma_{i}}=\left[\begin{array}{llll}
\boldsymbol{G}_{1} & \boldsymbol{G}_{\gamma} & \ldots & \boldsymbol{G}_{N_{\Gamma}}
\end{array}\right]
\end{aligned}
$$

onde $\sum, \boldsymbol{H}$ e $\boldsymbol{G}$ são as sub-matrizes que reúnem as funções interpoladoras dos campos de tensão e deslocamento no domínio e deslocamento no contorno, respectivamente; $N_{S}$ e $N_{U}$ são respectivamente os números de nós de tensão e deslocamento no domínio do elemento $\boldsymbol{e}$ e $N_{\Gamma}$ é o número de nós do elemento que pertencem ao lado $\boldsymbol{i}$ do contorno. Assim, pode-se ter uma representação geral das dimensões das matrizes representadas pelas eq.(4.11), eq.(4.12), eq.(4.13) e eq.(4.14):

$$
F_{e}=\left[\begin{array}{ccc}
F_{11} & \cdots & F_{1 N_{S}} \\
\vdots & \ddots & \vdots \\
F_{N_{S} 1} & \cdots & F_{N_{S} N_{S}}
\end{array}\right]
$$

onde:

$$
F_{\alpha \beta}=\int_{\Omega_{e}} \sum_{\alpha}^{T} f \sum_{\beta} d \Omega, \alpha, \beta=1, \ldots N_{S}
$$

Assim como: 


$$
A_{\Omega_{e}}=\left[\begin{array}{ccc}
A_{\Omega_{11}} & \cdots & A_{\Omega_{1 N_{U}}} \\
\vdots & \ddots & \vdots \\
A_{\Omega_{N_{S} 1}} & \cdots & A_{\Omega_{N_{S} N_{U}}}
\end{array}\right]
$$

onde:

$$
A_{\Omega_{\alpha \delta}}=\int_{\Omega_{e}}\left(L \sum_{\alpha}\right)^{T} H_{\delta} d \Omega, \alpha=1, \ldots N_{S}, \delta=1, \ldots N_{U}
$$

No contorno, tem-se:

$$
A_{\Gamma_{i}}=\left[\begin{array}{ccc}
A_{\Gamma_{11}} & \cdots & A_{\Gamma_{1 N_{\Gamma}}} \\
\vdots & \ddots & \vdots \\
A_{\Gamma_{N_{S} 1}} & \cdots & A_{\Gamma_{N_{S} N_{\Gamma}}}
\end{array}\right]
$$

onde :

$$
A_{\Gamma_{\alpha \gamma}}=\int_{\Gamma_{I}}\left(N \sum_{\alpha}\right)^{T} G \gamma d \Gamma, \alpha=1, \ldots N_{S}, \gamma=1, \ldots N_{\Gamma}
$$

Por outro lado, as forças generalizadas no contorno são dadas por:

$$
\boldsymbol{Q}_{\Gamma_{i}}=\left[\begin{array}{c}
\boldsymbol{Q}_{\Gamma_{1}} \\
\vdots \\
\boldsymbol{Q}_{\Gamma_{\gamma}} \\
\vdots \\
\boldsymbol{Q}_{\Gamma_{N_{\Gamma}}}
\end{array}\right]
$$

onde:

$$
Q_{\Gamma_{\gamma}}=\int_{\Gamma_{i i}} G_{\gamma}^{T}(\bar{t}) d \Gamma, \gamma=1, \ldots N_{\Gamma}
$$

\subsection{Elementos Finitos Híbridos-Mistos Quadrangulares Bilineares}

Restringindo-se a abordagem às analises planas, serão adotados neste trabalho, como elementos da malha de cobertura, os elementos finitos isoparamétricos convencionais de quatro nós. Como funções aproximadoras dos campos de tensões e deslocamentos no domínio $\Omega_{e}$ serão assumidas as funções bilineares convencionais utilizadas no MEF clássico. Para a interpolação do campo de deslocamento no contorno $\Gamma_{i}$, funções lineares serão aplicadas. 
Definindo-se $\varphi_{\alpha}, \alpha=\mathbf{1 , 2 , 3 , 4}$, como as funções bilineares e $\psi_{\gamma}, \gamma=\mathbf{1 , 2}$, como as lineares, então as eq.(4.15), eq.(4.16) e eq.(4.17), são escritas da seguinte forma:

$$
\begin{aligned}
S_{\Omega_{e}} & =\left[\begin{array}{llll}
\varphi_{1} I_{3} & \varphi_{2} I_{3} & \varphi_{3} I_{3} & \varphi_{4} I_{3}
\end{array}\right] \\
U_{\Omega_{e}} & =\left[\begin{array}{llll}
\varphi_{1} I_{2} & \varphi_{2} I_{2} & \varphi_{3} I_{2} & \varphi_{4} I_{2}
\end{array}\right] \\
U_{\Gamma_{i}} & =\left[\begin{array}{lll}
\psi_{1} I_{2} & \psi_{2} I_{2}
\end{array}\right]
\end{aligned}
$$

onde $I_{3}$ e $I_{2}$ são as matrizes identidades de terceira e segunda ordem respectivamente, uma vez que em cada nó definem-se três graus de liberdade de tensão e dois de deslocamento.

As funções bilineares para elementos finitos quadrilaterais regulares com um sistema de referência cartesiana localizado no centro do elemento têm a seguinte apresentação:

$$
\begin{gathered}
\varphi_{1}=\frac{1}{4}(X-1)(Y-1) \\
\varphi_{2}=-\frac{1}{4}(X+1)(Y-1) \\
\varphi_{3}=-\frac{1}{4}(X-1)(Y+1) \\
\varphi_{4}=\frac{1}{4}(X+1)(Y+1)
\end{gathered}
$$

onde $X=2\left(\frac{x}{a}\right)$ e $\boldsymbol{Y}=2\left(\frac{y}{b}\right)$ são coordenadas adimensionais variando entre -1 e 1 , enquanto $\boldsymbol{a}$ e $\boldsymbol{b}$ são as dimensões dos lados do elemento retangular apresentado na figura 4.1 . 


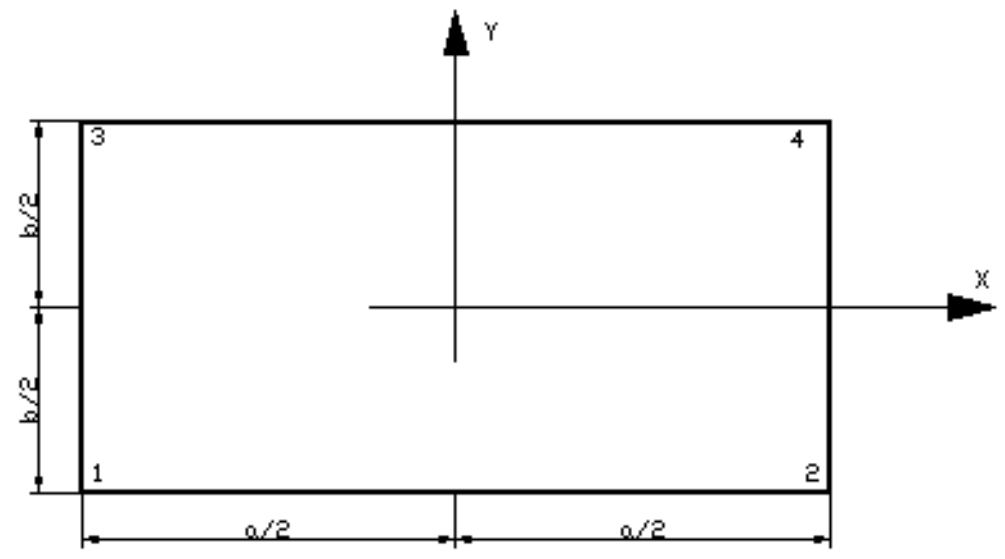

Figura 4.1 - Elemento finito quadrilateral com sistema de referência no baricentro.

Em termos gerais, pode-se escrever as funções de forma da seguinte maneira:

$$
\varphi_{\alpha}=\frac{1}{4} X_{\alpha} Y_{\alpha}\left(X+X_{\alpha}\right)\left(Y+Y_{\alpha}\right), \alpha=1,2,3,4
$$

onde $\boldsymbol{X}_{\alpha}, \boldsymbol{Y}_{\alpha}$ são as coordenadas adimensionais dos nós, ou seja, $\{(-1,-1),(1,-1),(-1,1),(1,1)\}$.

Assim para cada elemento no domínio $\Omega$, pode-se escrever:

$$
F_{e}=\left[\begin{array}{ccc}
F_{11} & \ldots & F_{14} \\
\vdots & \ddots & \vdots \\
F_{41} & \cdots & F_{44}
\end{array}\right]
$$

onde:

$$
F_{\alpha \beta}=\left(\int_{\Omega_{e}} \varphi_{\alpha} \varphi_{\beta} d \Omega\right) f I_{3}, \alpha, \beta=1, \ldots 4
$$

da mesma forma:

$$
A_{\Omega_{e}}=\left[\begin{array}{ccc}
A_{V_{11}} & \cdots & A_{V_{14}} \\
\vdots & \ddots & \vdots \\
A_{V_{41}} & \cdots & A_{V_{44}}
\end{array}\right]
$$

onde: 


$$
A_{\Omega_{\alpha \beta}}=\left[\begin{array}{cc}
\int_{\Omega_{e}} \frac{\partial \varphi_{\alpha}}{\partial x} \varphi_{\beta} d \Omega I_{2} & 0 \\
0 & \int_{\Omega_{e}} \frac{\partial \varphi_{\alpha}}{\partial y} \varphi_{\beta} d \Omega I_{2} \\
\int_{\Omega_{e}} \frac{\partial \varphi_{\alpha}}{\partial y} \varphi_{\beta} d \Omega I_{2} & \int_{\Omega_{e}} \frac{\partial \varphi_{\alpha}}{\partial x} \varphi_{\beta} d \Omega I_{2}
\end{array}\right], \alpha, \beta=1, \ldots 4 .
$$

Nas relações anteriores:

$$
d \Omega=d x d y=\frac{a b}{4} d X d Y
$$

Para a integração numérica das matrizes da eq.(4.34) e da eq.(4.36) se utilizam dois pontos de Gauss em cada direção. Neste caso particular, as integrais das eq.(4.35) e eq.(4.37) têm a forma exata fornecida por:

$$
\begin{aligned}
& \int_{\Omega_{e}} \varphi_{\alpha} \varphi_{\beta} d \Omega=\frac{a b}{4} \int_{-1}^{1} \int_{-1}^{1} \varphi_{\alpha} \varphi_{\beta} d X d Y=\frac{1}{16} a b\left(\frac{1}{3} X_{\alpha} X_{\beta}+1\right)\left(\frac{1}{3} Y_{\alpha} Y_{\beta}+1\right) \\
& \int_{\Omega_{e}} \frac{\partial \varphi_{\alpha}}{\partial x} \varphi_{\beta} d \Omega=\left(\frac{X_{\alpha} Y_{\alpha}}{8}\right)\left(\frac{1}{3} b Y_{\beta}+b Y_{\alpha}\right) \\
& \int_{\Omega_{e}} \frac{\partial \varphi_{\alpha}}{\partial y} \varphi_{\beta} d \Omega=\left(\frac{X_{\alpha} Y_{\alpha}}{8}\right)\left(\frac{1}{3} a X_{\beta}+a X_{\alpha}\right)
\end{aligned}
$$

As funções lineares para os lados dos elementos finitos quadrilaterais que pertencem ao contorno $\Gamma$ têm a seguinte representação:

$$
\begin{gathered}
\psi_{1}=-\frac{1}{2}(\xi-1) \\
\psi_{2}=\frac{1}{2}(\xi+1)
\end{gathered}
$$

onde $\xi$ é a coordenada adimensional com origem no centro do elemento e variando entre -1 e 1 .

Para cada lado do elemento finito retangular que pertence ao contorno $\Gamma$, podese escrever: 


$$
A_{\Gamma_{i}}=\left[\begin{array}{ccc}
A_{\Gamma_{11}} & \ldots & A_{\Gamma_{12}} \\
\vdots & \ddots & \vdots \\
A_{\Gamma_{11}} & \cdots & A_{\Gamma_{42}}
\end{array}\right],
$$

onde:

$$
A_{\Gamma_{\alpha \gamma}}=\left[\begin{array}{cc}
\int_{\Gamma_{i}} n_{x} \varphi_{\alpha} \psi_{\gamma} d \Gamma I_{2} & 0 \\
0 & \int_{\Gamma_{i}} n_{y} \varphi_{\alpha} \psi_{\gamma} d \Gamma I_{2} \\
\int_{\Gamma_{i}} n_{y} \varphi_{\alpha} \psi_{\gamma} d \Gamma I_{2} & \int_{\Gamma_{i}}^{n} n_{x} \varphi_{\alpha} \psi_{\gamma} d \Gamma I_{2}
\end{array}\right], \alpha=1, \ldots 4, \gamma=1, \ldots 2
$$

Vale salientar que para nós $\alpha$ do elemento $e$ que não pertencem ao lado $\Gamma_{i}$ a matriz $A_{\Gamma_{a \gamma}}$ é nula.

As forças generalizadas no contorno são dadas por:

$$
\boldsymbol{Q}_{\Gamma_{i}}=\left[\begin{array}{l}
\boldsymbol{Q}_{\Gamma_{1}} \\
\boldsymbol{Q}_{\Gamma_{2}}
\end{array}\right]
$$

onde:

$$
Q_{\Gamma_{\gamma}}=\left[\begin{array}{l}
\int_{\Gamma_{i}} \psi_{\gamma} \bar{t}_{x} d \Gamma I_{2} \\
\int_{\Gamma_{i}} \psi_{\gamma} \bar{t}_{y} d \Gamma I_{2}
\end{array}\right], \gamma=\mathbf{1 , 2}
$$

Para a integração numérica das matrizes da eq.(4.44) e da eq.(4.46) se utilizam dois pontos de Gauss. Nota-se, entretanto, que as componentes da normal e as funções $\varphi$ e $\psi$ que aparecem nessas integrais dependem do lado do elemento quadrilateral. Então:

- se o lado horizontal superior do elemento é o que coincide com o contorno $\Gamma$, têm-se:

$$
\begin{aligned}
& n_{x}=\mathbf{0} \\
& n_{y}=1 \\
& X=2 \frac{x}{a} \\
& Y=1 \\
& \xi=X
\end{aligned}
$$


- se o lado horizontal inferior do elemento é o que coincide com o contorno $\Gamma$, têm-se:

$$
\begin{aligned}
& n_{x}=0 \\
& n_{y}=-1 \\
& X=2 \frac{x}{a} \\
& Y=-1 \\
& \xi=X
\end{aligned}
$$

- se o lado vertical direito do elemento é o que coincide com o contorno $\Gamma$, têm-se:

$$
\begin{aligned}
& n_{x}=1 \\
& n_{y}=0 \\
& X=1 \\
& Y=2 \frac{y}{b} \\
& \xi=Y
\end{aligned}
$$

- se o lado vertical esquerdo do elemento é o que coincide com o contorno $\Gamma$, têm-se:

$$
\begin{aligned}
& n_{x}=-1 \\
& n_{y}=0 \\
& X=-1 \\
& Y=2 \frac{y}{b} \\
& \xi=Y
\end{aligned}
$$

A integração numérica das matrizes da eq.(4.44) e da eq.(4.46) será aqui exemplificada somente para um dos casos mostrado acima. Considerando-se então o lado vertical direito do elemento pode-se escrever:

$$
\begin{aligned}
& \psi_{1}=-\frac{1}{2}(Y-1) \\
& \psi_{2}=\frac{1}{2}(Y+1)
\end{aligned}
$$


Com a utilização de dois pontos de Gauss, as integrais das eq.(4.45) e eq.(4.47), têm a forma exata fornecida por:

$$
\begin{aligned}
& \int_{\Gamma_{i}} \varphi_{\alpha} \psi_{\gamma} d \Gamma=\left(\frac{X_{\alpha}+1}{3}\right) \\
& \int_{\Gamma_{i}} \psi_{\gamma} \bar{t}_{x} d \Gamma=2 \bar{t}_{x} \\
& \int_{\Gamma_{i}} \psi_{\gamma} \bar{t}_{y} d \Gamma=2 \bar{t}_{y}
\end{aligned}
$$




\section{FHMT com Enriquecimento - Método dos Elementos Finitos Generalizados (MEFG)}

\subsection{O Método dos Elementos Finitos Generalizados (MEFG)}

\subsubsection{Origem}

Os métodos sem malha (ver apêndice B) apresentam certa dificuldade no controle do erro da integração numérica, aliado ainda, à necessidade freqüente do uso de técnicas especiais (como por exemplo, os multiplicadores de Lagrange) para aplicação das condições de contorno essenciais do problema.

Para superar essas dificuldades, percebeu-se a possibilidade de combinar o MEF clássico com as técnicas de enriquecimento dos métodos sem malha. Surgiu então o Método dos Elementos Finitos Generalizados (MEFG).

Assim, o Método dos Elementos Finitos Generalizados (MEFG) pode ser entendido como uma combinação da forma clássica do Método dos Elementos Finitos (MEF) com técnicas dos métodos sem malha, especificamente a estratégia de enriquecimento da aproximação adotada no Método das Nuvens "hp".

Segundo o trabalho de Duarte e Babuška e Oden (2000), o MEFG, foi proposto independentemente por:

- Babuška e Caloz e Osborn (1994) sob a denominação de Método dos Elementos Finitos Especiais;

- Melenk e Babuška (1996) e Babuška e Melenk (1997) como Método dos Elementos Finitos Partição Unidade (MEFPU);

- Duarte (1996), Duarte e Oden (1995, 1996a, 1996b) com os trabalhos correspondentes à formulação do Método das Nuvens;

- Oden e Duarte e Zienkiewicz (1998) como uma formulação híbrida entre o Método das Nuvens "hp" e a forma clássica do MEF. 
A fundamentação do MEFG vem com os seguintes trabalhos:

- De Oden e Duarte e Zienkiewicz (1998), onde foi apresentada a possibilidade de empregar a malha de elementos finitos para a definição dos nós e das nuvens, e realizar o enriquecimento, à maneira do Método das Nuvens "hp", sobre as funções de forma Lagrangianas usadas no MEF clássico (que constituem uma partição da unidade, se relaxadas algumas das condições que definem uma PU).

- De Strouboulis e Babuška e Cops (2000), que apresentam o MEFG como combinação do MEF clássico com o Método dos Elementos Finitos Partição da Unidade, (MELENK,1992,1995; BABUŠKA; MELENK,1996). A principal característica desse trabalho é a adição de funções solução do problema de valor de contorno estudado ao espaço da formulação clássica do MEF.

Nota-se, por esses trabalhos, que as características principais do MEFG já estavam bem definidas, ou seja: utilização da malha de cobertura como domínio de integração, uso das funções de forma Lagrangianas do MEF convencional como PU e seu enriquecimento (utilizando as estratégia do Método das Nuvens "hp") com funções polinomiais ou não. A região de influência de um certo nó, denominada nuvem ou suporte, é definida pelo conjunto de elementos finitos que têm por vértice comum o nó em questão. Vale ressaltar ainda, que nessas condições não existem problemas quanto à imposição das condições de contorno do problema.

Algumas aplicações do MEFG são encontradas nos trabalhos de Duarte e Babuška e Oden (2000) e Strouboulis e Babuška e Cops (2000). Os dois trabalhos evidenciam as vantagens do MEFG com relação ao MEF clássico na análise de estruturas complexas, caracterizadas por cantos reentrantes que se constituem em pontos de singularidade (região de grandes gradientes de tensão).

\subsection{FHMT com Enriquecimento Nodal.}

\subsubsection{Considerações Iniciais}

No Método das Nuvens "hp" a nuvem $\omega_{j}$ atrelada a um nó $\boldsymbol{x}_{\boldsymbol{j}}$, é definida como uma região circular (ou poligonal) em torno daquele nó. Já na FHMT com 
enriquecimento, aproveita-se, como no MEFG clássico, a malha de cobertura para definição das nuvens ou suportes. Como na FHMT há uma malha de cobertura para o domínio e outra para o contorno, existem, também, suportes ou nuvens para o domínio e contorno do problema. Em ambas as malhas, a nuvem em torno de um nó é formada pelo conjunto de elementos que têm aquele nó como vértice, como indica a figura 5.1.

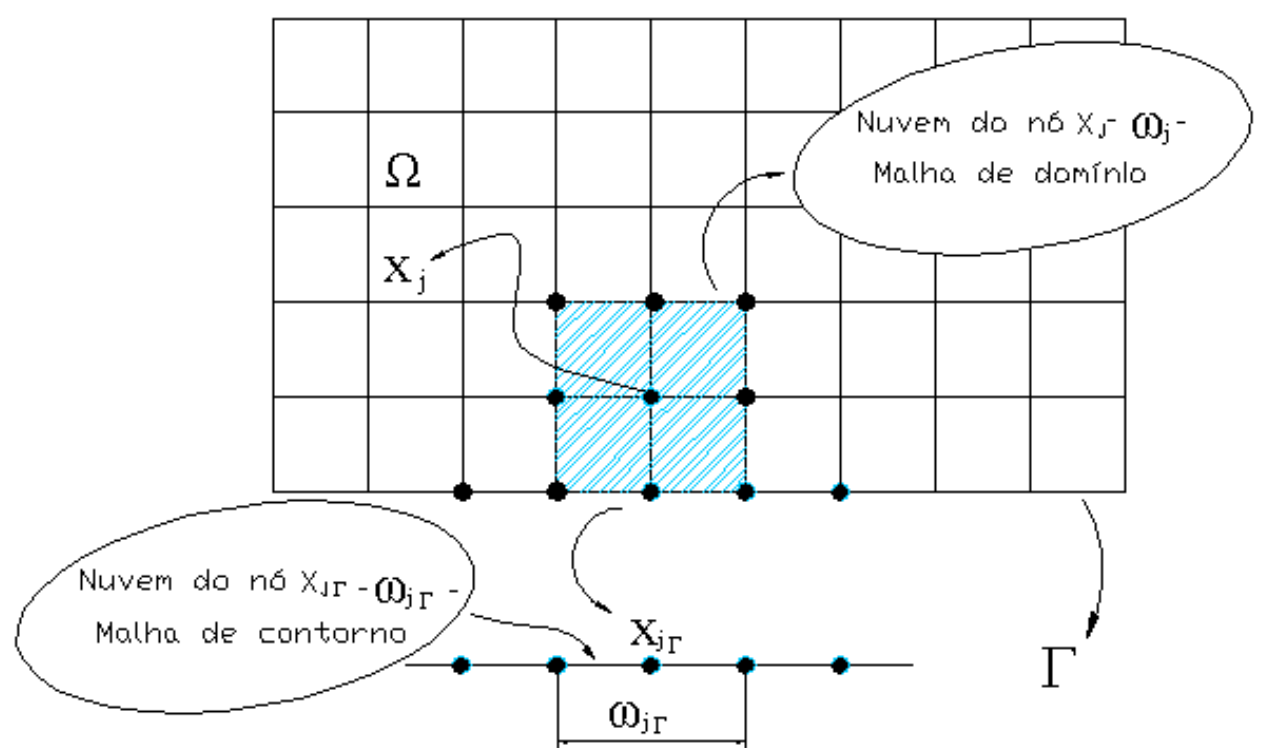

Figura 5.1 - Nuvens de influência para as malhas de cobertura: domínio (bidimensional) e contorno (unidimensional).

Em cada nuvem, a aproximação nodal fica determinada aproveitando-se as funções de forma dos elementos finitos que compõe a nuvem e associadas ao nó base. Dessa forma, a continuidade entre elementos será da mesma ordem das funções adotadas para aproximação. Empregando-se o mesmo procedimento proposto no Método das Nuvens "hp", o enriquecimento sobre as funções de forma nodais (para cada um dos campos aproximados na FHMT), em cada nuvem, é realizado pela sua multiplicação, por polinômios ou outras funções especiais. Assim, pode-se representar:

- A família de funções para o campo de tensões no domínio $\Omega$ :

$$
\left.\Im_{N}^{p}=\left\{S_{\Omega_{j}}\right\}_{j=1}^{N} \cup\left\{S_{\Omega_{j}} L_{j n_{e}}\right\}_{j=1}^{N}: j=1, \ldots, N ; n_{e}=1, \ldots, I(j)\right\}
$$

- A família de funções para o campo de deslocamentos no domínio $\Omega$ :

$$
\left.\Theta_{N}^{p}=\left\{U_{\Omega_{j}}\right\}_{j=1}^{N} \cup\left\{U_{\Omega_{j}} M_{j n_{e}}\right\}_{j=1}^{N}: j=1, \ldots, N ; n_{e}=1, \ldots, I(j)\right\}
$$


- A família de funções para o campo de deslocamentos no contorno $\Gamma$ :

$$
\left.\Xi_{N_{\Gamma}}^{p}=\left\{U_{\Gamma_{j}}\right\}_{j=1}^{N_{\Gamma}} \cup\left\{U_{\Gamma_{j}} \boldsymbol{O}_{j n_{e_{\Gamma}}}\right\}_{j=1}^{N_{\Gamma}}: j=1, \ldots, N_{\Gamma} ; \boldsymbol{n}_{e_{\Gamma}}=1, \ldots, I(j)\right\}
$$

onde para as representações anteriores, têm-se que:

- $N$ é o número total de nós no domínio $\Omega$;

- $N_{\Gamma}$ é o número total de nós no contorno $\Gamma$;

- $\boldsymbol{p}$ é o grau máximo da aproximação Lagrangiana resultante;

- $\boldsymbol{S}_{\Omega_{j}}$ e $\boldsymbol{U}_{\Omega_{j}}$ são as funções de forma (Partições da Unidade) para os campos de tensão e deslocamento respectivamente, referentes aos nós $\boldsymbol{j}=\mathbf{1}, \ldots, \boldsymbol{N}$ do domínio $\Omega$;

- $U_{\Gamma_{j}}$ é a função de forma (Partição da Unidade) para o campo de deslocamento referente dos nós do contorno $\Gamma\left(j=1, \ldots, N_{\Gamma}\right)$;

- $\boldsymbol{L}_{\boldsymbol{j} \boldsymbol{n}_{e}}, \boldsymbol{M}_{\boldsymbol{j} \boldsymbol{n}_{e}}$ são as $\boldsymbol{n}_{\boldsymbol{e}}$ funções que multiplicam ou enriquecem a função de forma de domínio definida em cada nó de índice $\boldsymbol{j}$;

- $\boldsymbol{O}_{\boldsymbol{j n}_{\boldsymbol{e}_{\Gamma}}}$ são as $\boldsymbol{n}_{e_{\Gamma}}$ funções que multiplicam (enriquecem) a função de forma de contorno definida em cada nó de índice $\boldsymbol{j}$;

- $I(j)$ é o contador para o número de funções adicionadas a cada nó de índice j.

\subsection{Elementos Finitos Híbridos-Mistos de Tensão Quadrangulares Bilineares com Enriquecimento Nodal}

\subsubsection{Introdução}

Na FHMT são aproximados três campos independentes e, como já salientado, o enriquecimento também pode ser realizado de forma independente para os três campos. Neste item diferentes possibilidades de enriquecimento são apresentadas. Inicialmente, a estrutura de enriquecimento é desenvolvida com ajuda de funções polinomiais. Em seguida, a estratégia de enriquecimento é apresentada utilizando-se especificamente funções relacionadas às soluções fornecidas pela Mecânica da Fratura Elástica Linear. Uma terceira opção apresentada é o emprego de funções enriquecedoras, das bases aproximativas das tensões, que geram estados de tensões auto-equilibrados. Finalmente, 
funções trigonométricas são propostas como funções enriquecedoras das funções de forma da FHMT.

\subsubsection{Enriquecimento Nodal Polinomial}

Supondo que num domínio plano tenha sido adotada uma malha de elementos quadrangulares de quatro nós, então funções Lagrangianas bilineares são utilizadas para aproximação dos campos de tensão e deslocamento no domínio. Admitindo-se que se deseja enriquecer a aproximação correspondente a um nó $\alpha$ da malha de cobertura, com ajuda de funções polinomiais $\boldsymbol{h}_{\boldsymbol{k} \alpha}, \boldsymbol{k} \alpha=\mathbf{1}, \ldots, \boldsymbol{n}_{\boldsymbol{e}}$, onde $\boldsymbol{n}_{\boldsymbol{e}}$ é o número de funções de enriquecimento escolhidas as eq.(5.1) e eq.(5.2) podem ser re-escritas da seguinte forma:

- A família de funções para o campo de tensões no domínio $\Omega$ :

$$
\mathfrak{I}_{N}^{2}=\left\{\left\{S_{\Omega_{j}}\right\}_{j=1}^{N} \cup\left\{S_{\Omega_{j}} \boldsymbol{h}_{j n_{e}}\right\}_{j=1}^{N}: j=1, \ldots, N ; \boldsymbol{n}_{e}=1, \ldots, I(j)\right\}
$$

utilizada para construir a seguinte aproximação:

$$
\hat{\sigma}=\sum_{j=1}^{N} \boldsymbol{S}_{\Omega_{j}}\left\{\boldsymbol{s}_{\Omega_{j}}+\sum_{i=1}^{n_{e}} \boldsymbol{h}_{j i} \boldsymbol{b}_{j i}\right\}
$$

onde $s_{\Omega_{j}}$ são os graus de liberdade de tensões associadas às funções de forma originais e $\boldsymbol{b}_{j i}$ são os novos parâmetros nodais correspondentes a cada uma das parcelas de enriquecimento.

- A família de funções para o campo de deslocamentos no domínio $\Omega$ :

$$
\Theta_{N}^{2}=\left\{\left\{U_{\Omega_{j}}\right\}_{j=1}^{N} \cup\left\{U_{\Omega_{j}} \boldsymbol{h}_{j n_{e}}\right\}_{j=1}^{N}: j=1, \ldots, N ; \boldsymbol{n}_{e}=1, \ldots, I(j)\right\}
$$

utilizada para construir a seguinte aproximação:

$$
\hat{\boldsymbol{u}}=\sum_{j=1}^{N} \boldsymbol{U}_{\Omega_{j}}\left\{\boldsymbol{u}_{\Omega_{j}}+\sum_{i=1}^{n_{e}} \boldsymbol{h}_{j i} \boldsymbol{c}_{j i}\right\}
$$


onde $\boldsymbol{u}_{\Omega_{j}}$ são graus de liberdade em deslocamento associados às funções de forma originais e $c_{j i}$ são os novos parâmetros nodais correspondentes a cada uma das parcelas de enriquecimento.

Para a malha de contorno são normalmente empregados elementos retos de dois nós com funções de forma Lagrangianas lineares. Analogamente, supondo que se deseja enriquecer a aproximação correspondente a um nó $\alpha_{\Gamma}$ da malha de cobertura de contorno com ajuda de funções polinomiais $\boldsymbol{h}_{\boldsymbol{k} \alpha_{\Gamma}}, \boldsymbol{k} \boldsymbol{\alpha}_{\Gamma}=\mathbf{1}, \ldots, \boldsymbol{n}_{\boldsymbol{e}_{\Gamma}}, \boldsymbol{n}_{\boldsymbol{e}_{\Gamma}}$ é o número de funções de enriquecimento adotadas, a eq.(5.3) pode ser re-escrita da seguinte forma:

- A família de funções para o campo de deslocamentos no contorno $\Gamma$ :

$$
\left.\Xi_{N_{\Gamma}}^{1}=\left\{U_{\Gamma j}\right\}_{j=1}^{N_{\Gamma}} \cup\left\{U_{\Gamma j} \boldsymbol{h}_{j n_{e_{\Gamma}}}\right\}_{j=1}^{N_{\Gamma}}: j=1, \ldots, N_{\Gamma} ; n_{e_{\Gamma}}=1, \ldots, I(j)\right\}
$$

utilizada para construir a seguinte aproximação:

$$
\hat{\boldsymbol{u}}_{\Gamma}=\sum_{j=1}^{N_{\Gamma}} \boldsymbol{U}_{\Gamma_{j}}\left\{\boldsymbol{u}_{\Gamma_{j}}+\sum_{i=1}^{n_{e_{\Gamma}}} \boldsymbol{h}_{j i} \boldsymbol{d}_{j i}\right\}
$$

onde $\boldsymbol{u}_{\Gamma_{j}}$ são graus de liberdade em deslocamento associados às funções de forma originais e $\boldsymbol{d}_{j i}$ são os novos parâmetros nodais correspondentes a cada uma das parcelas de enriquecimento.

Uma opção conveniente para as funções $\boldsymbol{h}_{\boldsymbol{k} \alpha}$ e $\boldsymbol{h}_{\boldsymbol{k} \alpha_{\Gamma}}$ escolhidas para o enriquecimento, é que sejam tais que:

$$
\begin{aligned}
& \boldsymbol{h}_{k \alpha}\left(\boldsymbol{X}_{\alpha}, \boldsymbol{Y}_{\alpha}\right)=\mathbf{0} \\
& \boldsymbol{h}_{k \alpha_{\Gamma}}\left(\xi_{\alpha_{\Gamma}}\right)=\mathbf{0}
\end{aligned}
$$

onde $\boldsymbol{X}_{\alpha}, \boldsymbol{Y}_{\alpha}$ são as coordenadas adimensionais para o elemento da malha de cobertura de domínio e $\xi_{\alpha_{\Gamma}}$ é a coordenada adimensional para o elemento da malha de cobertura de contorno. Nesse caso, essas funções são nulas no nó enriquecido ('funções bolhas') e a vantagem desse procedimento é que se preserva o significado físico de graus de liberdade nodais $\boldsymbol{s}_{\Omega}, \boldsymbol{u}_{\Omega}$ e $\boldsymbol{u}_{\Gamma}$. 
Apesar de toda a estrutura de nuvens centradas em nós, características do MEFG, todo a implementação computacional se realiza sobre uma estrutura convencional do MEF, acrescentando-se as matrizes de cada elemento um número de linhas e colunas correspondentes aos enriquecimentos realizados sobre cada um de seus nós.

Assim sendo, as matrizes de interpolação dos campos, apresentadas nas eq.(4.26), eq.(4.27) e eq.(4.28), podem ser escritas da seguinte forma:

$$
\begin{aligned}
S_{\Omega_{e}} & =\left[\begin{array}{llll}
\varphi_{1} \Delta_{1} & \varphi_{2} \Delta_{2} & \varphi_{3} \Delta_{3} & \varphi_{4} \Delta_{4}
\end{array}\right] \\
U_{\Omega_{e}} & =\left[\begin{array}{llll}
\varphi_{1} \Delta_{1} & \varphi_{2} \Delta_{2} & \varphi_{3} \Delta_{3} & \varphi_{4} \Delta_{4}
\end{array}\right] \\
U_{\Gamma i} & =\left[\begin{array}{lll}
\psi_{1} \Delta_{\Gamma_{1}} & \psi_{2} \Delta_{\Gamma_{2}}
\end{array}\right]
\end{aligned}
$$

onde $\Delta_{\alpha}, \alpha=1, \ldots, 4$ e $\Delta_{\Gamma_{\alpha_{\Gamma}}}, \alpha_{\Gamma}=1,2$ são, respectivamente, as matrizes de enriquecimento polinomial do nó $\alpha$ do elemento de domínio e do nó $\alpha_{\Gamma}$ do elemento de contorno.

Considere-se agora que as matrizes de enriquecimento polinomial sejam dadas por:

$$
\Delta_{\alpha}=\left[\begin{array}{llllll}
I_{3} & h_{1 \alpha} I_{3} & \ldots & h_{k \alpha} I_{3} & \ldots & h_{n_{\alpha} \alpha} I_{3}
\end{array}\right]
$$

quando do enriquecimento do campo de tensões;

$$
\Delta_{\alpha}=\left[\begin{array}{lllllll}
I_{2} & h_{1 \alpha} I_{2} & \ldots & h_{k \alpha} I_{2} & \ldots & h_{n_{\alpha} \alpha} I_{2}
\end{array}\right]
$$

quando do enriquecimento do campo de deslocamentos de domínio; e

$$
\Delta_{\Gamma_{\alpha_{\Gamma}}}=\left[\begin{array}{llllll}
I_{2} & h_{1 \alpha_{\Gamma}} I_{2} & \ldots & h_{k \alpha_{\Gamma}} I_{2} & \ldots & h_{n_{\alpha \Gamma} \alpha_{\Gamma}} I_{2}
\end{array}\right]
$$

quando do enriquecimento do campo de deslocamentos no contorno.

Claramente, se as funções $\boldsymbol{h}_{\boldsymbol{k} \alpha}$ e $\boldsymbol{h}_{\boldsymbol{k} \alpha_{\Gamma}}$ são nulas, preserva-se, com as matrizes $\boldsymbol{I}_{2}$ e $\boldsymbol{I}_{3}$, a estrutura convencional do MEF.

Observa-se que formas habituais para as funções bolhas $\boldsymbol{h}_{\boldsymbol{k} \alpha}$ e $\boldsymbol{h}_{\boldsymbol{k} \alpha_{\Gamma}}$ são:

$$
\boldsymbol{h}_{k \alpha}=\left(X-X_{\alpha}\right),\left(X-X_{\alpha}\right)^{2},\left(X-X_{\alpha}\right)\left(Y-Y_{\alpha}\right),\left(Y-Y_{\alpha}\right)^{2}, \ldots
$$




$$
\boldsymbol{h}_{k \alpha_{\Gamma}}=\left(\xi-\xi_{\alpha_{\Gamma}}\right),\left(\xi-\xi_{\alpha_{\Gamma}}\right)^{2}, \ldots
$$

Assim para qualquer nó $\alpha,(\alpha=1, \ldots, 4)$ do elemento finito retangular $e$, escolhido para ser enriquecido, pode-se escrever:

- Para a matriz representada pela eq.(4.11)

$$
\begin{aligned}
& S_{\Omega_{e}}^{T} f S_{\Omega_{e}}=\left[\varphi_{\alpha} \varphi_{\beta} \Delta_{\alpha}^{T} f \Delta_{\beta}\right], \alpha=\beta=1, \ldots 4 \\
& F_{e}=\left\lfloor F_{\alpha \beta}\right\rfloor \alpha=\beta=1, \ldots 4
\end{aligned}
$$

onde:

$$
F_{\alpha \beta}=\left(\int_{\Omega_{e}} \varphi_{\alpha} \varphi_{\beta} \Delta_{\alpha}^{T} f \Delta_{\beta} d \Omega\right), \alpha, \beta=1, \ldots 4
$$

Nota-se que:

$$
\Delta_{\alpha}^{T} f \Delta_{\beta}=\left[\begin{array}{ccccc}
f & \ldots & h_{k \beta} f & \ldots & h_{n_{e} \beta} f \\
\vdots & \ddots & \vdots & \ddots & \vdots \\
h_{k \alpha} f & \cdots & h_{k \alpha} h_{k \beta} f & \cdots & h_{k \alpha} h_{n_{e} \beta} f \\
\vdots & \ddots & \vdots & \ddots & \vdots \\
h_{n_{e} \alpha} f & \cdots & h_{n_{e} \alpha} f & \cdots & h_{n_{e} \alpha} h_{n_{e} \beta} f
\end{array}\right]
$$

Da mesma forma, têm-se:

- Para a matriz representada pela eq.(4.12)

$$
\begin{aligned}
& L S_{\Omega_{e}}=L\left[\begin{array}{llll}
\varphi_{1} \Delta_{1} & \varphi_{2} \Delta_{2} & \varphi_{3} \Delta_{3} & \varphi_{4} \Delta_{4}
\end{array}\right]= \\
& =\left[\begin{array}{lll}
\left(L \varphi_{1}\right) \Delta_{1}+\varphi_{1}\left(L \Delta_{1}\right) & \ldots & \left(L \varphi_{4}\right) \Delta_{4}+\varphi_{4}\left(L \Delta_{4}\right)
\end{array}\right] \\
& \left(L S_{\Omega_{e}}\right)^{T}=\left[\begin{array}{c}
\Delta_{l}^{T}\left(L \varphi_{1}\right)^{T}+\varphi_{1}\left(L \Delta_{1}\right)^{T} \\
\vdots \\
\Delta_{4}^{T}\left(L \varphi_{4}\right)^{T}+\varphi_{4}\left(L \Delta_{4}\right)^{T}
\end{array}\right] \\
& \left(L S_{\Omega_{e}}\right)^{T} U_{\Omega_{e}}=\left(\Delta_{\alpha}^{T}\left(L \varphi_{\alpha}\right)^{T}+\varphi_{\alpha}\left(L \Delta_{\alpha}\right)\right)\left(\varphi_{\beta} \Delta_{\beta}\right), \alpha=\beta=1, \ldots, 4 \\
& A_{\Omega_{e}}=\left\lfloor A_{\Omega_{\alpha \beta}}\right\rfloor \alpha=\beta=1, \ldots 4
\end{aligned}
$$

onde: 


$$
A_{\Omega_{\alpha \beta}}=\left(\int_{\Omega_{e}}\left(\Delta_{\alpha}^{T}\left(L \varphi_{\alpha}\right)^{T}+\varphi_{\alpha}\left(L \Delta_{\alpha}\right)\right)\left(\varphi_{\beta} \Delta_{\beta}\right) d \Omega\right), \alpha, \beta=1, \ldots 4
$$

Para qualquer nó $\alpha_{\Gamma}\left(\alpha_{\Gamma}=1,2\right)$, de cada lado do elemento finito retangular que pertence ao contorno $\Gamma$, que é escolhido para ser enriquecido, pode-se escrever:

- Para a matriz representada pela eq.(4.13)

$$
\begin{aligned}
& N S_{\Omega_{e}}=\left[\begin{array}{llll}
\left(N \varphi_{1}\right) \Delta_{1} & \left(N \varphi_{2}\right) \Delta_{2} & \left(N \varphi_{3}\right) \Delta_{3} & \left(N \varphi_{4}\right) \Delta_{4}
\end{array}\right] \\
& \left(N S_{\Omega_{e}}\right)^{T}=\left[\begin{array}{c}
\Delta_{1}^{T}\left(N \varphi_{1}\right)^{T} \\
\vdots \\
\Delta_{4}^{T}\left(N \varphi_{4}\right)^{T}
\end{array}\right] \\
& \left(N S_{\Omega_{e}}\right)^{T} U_{\Gamma_{i}}=\left(\Delta_{\alpha}^{T}\left(N \varphi_{\alpha}\right)^{T}\right)\left(\psi_{\gamma} \Delta_{\Gamma_{\gamma}}\right), \alpha=1, \ldots, 4, \gamma=1,2 \\
& A_{\Gamma_{i}}=\left\lfloor A_{\Gamma_{\alpha \lambda}}\right\rfloor \alpha=1, \ldots 4, \gamma=1,2
\end{aligned}
$$

onde:

$$
A_{\Gamma \alpha \gamma}=\left(\int_{\Gamma_{i}}\left(\Delta_{\alpha}^{T}\left(N \varphi_{\alpha}\right)^{T}\right)\left(\psi_{\gamma} \Delta_{\Gamma_{\gamma}}\right) d \Gamma\right), \alpha=1, \ldots 4, \gamma=1,2,
$$

- Para a matriz representada pela eq.(4.14)

$$
\begin{gathered}
\boldsymbol{U}_{\Gamma_{i}}^{T}=\left[\begin{array}{l}
\psi_{1} \Delta_{\Gamma_{1}}^{T} \\
\psi_{2} \Delta_{\Gamma_{2}}^{T}
\end{array}\right] \\
\boldsymbol{Q}_{\Gamma_{i}}=\left[\boldsymbol{Q}_{\Gamma_{\gamma}}\right\rfloor \gamma=\mathbf{1 , 2}
\end{gathered}
$$

onde:

$$
Q_{\Gamma \gamma}=\left[\int_{\Gamma_{i}} \psi_{\gamma} \Delta_{\Gamma_{\gamma}}^{T} \bar{t} d \Gamma\right], \gamma=1,2
$$

Para o cálculo do número de pontos de Gauss necessários para integração das matrizes enriquecidas de cada elemento, consideram-se os casos:

- Para o domínio do problema:

Sendo $\boldsymbol{g}_{a p}$ o grau máximo dos polinômios $\boldsymbol{h}_{\boldsymbol{k} \alpha}$ em uma direção, então são necessários $\boldsymbol{g}_{a p}+2$ pontos de Gauss em cada direção para integração; 
- Para o contorno do problema:

Sendo $\boldsymbol{g}_{a p \Gamma}$ o grau máximo dos polinômios $\boldsymbol{h}_{\boldsymbol{k} \alpha_{\Gamma}}$, então são necessários $\boldsymbol{g}_{a p \Gamma}+2$ pontos de Gauss para integração;

Vale ressaltar que o enriquecimento de polinômios empregando-se bases também polinomiais pode gerar famílias de funções linearmente dependentes. Isso acarreta um grande problema, pois no sistema de equações representado pela eq.(3.32) já existem dependências lineares provenientes da própria formulação Híbrida-Mista de Tensão. Neste trabalho, adaptou-se o procedimento numérico de Babuška, sugerido por Strouboulis e Babuška e Copps (2000), para contornar esse problema. No apêndice A, apresenta-se esse procedimento detalhadamente.

\subsubsection{Enriquecimento Nodal com Funções que se Assemelham às Soluções da Mecânica da Fratura Elástica Linear}

Considere-se, no plano, a solução referente a uma trinca alinhada com o eixo y e tracionada na direção $\mathrm{x}$, conforme indicado na figura 5.2.

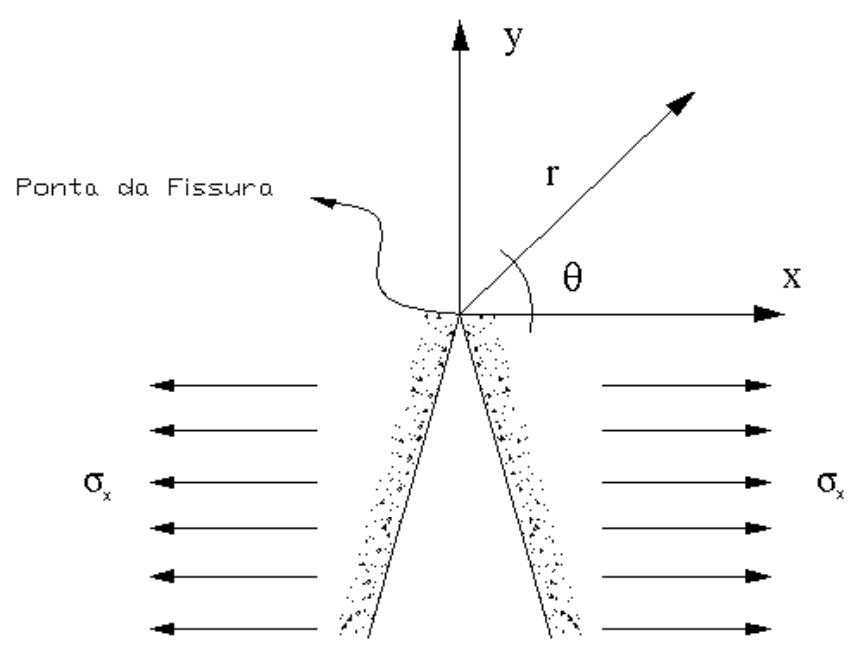

Figura 5.2 - Fissura em um campo tensional

A distribuição das tensões e o campo de deslocamentos próximos à ponta da fissura, fornecidos pela solução da Mecânica da Fratura Elástica Linear podem ser escritas como:

$$
\sigma_{x}^{\prime}=\frac{\sigma_{x} \sqrt{2 \pi}}{K_{I}}=\frac{1}{\sqrt{r}} \cos \left(\frac{\theta}{2}\right)\left[1+\operatorname{sen}\left(\frac{\theta}{2}\right) \operatorname{sen}\left(\frac{3 \theta}{2}\right)\right]
$$




$$
\begin{aligned}
& \sigma_{y}^{\prime}=\frac{\sigma_{y} \sqrt{2 \pi}}{K_{I}}=\frac{1}{\sqrt{r}} \cos \left(\frac{\theta}{2}\right)\left[1-\operatorname{sen}\left(\frac{\theta}{2}\right) \operatorname{sen}\left(\frac{3 \theta}{2}\right)\right] \\
& \tau_{x y}^{\prime}=\frac{\tau_{x y} \sqrt{2 \pi}}{K_{I}}=\frac{1}{\sqrt{r}} \cos \left(\frac{\theta}{2}\right)\left[-\operatorname{sen}\left(\frac{\theta}{2}\right) \operatorname{sen}\left(\frac{3 \theta}{2}\right)\right] \\
& u^{\prime}=\frac{u \sqrt{2 \pi}}{K_{I}}=\frac{-\sqrt{r}}{2 G}\left\{\left[k+\frac{1}{2}\right] \operatorname{sen}\left(\frac{\theta}{2}\right)-\frac{1}{2} \operatorname{sen}\left(\frac{3 \theta}{2}\right)\right\} \\
& v^{\prime}=\frac{v \sqrt{2 \pi}}{K_{I}}=\frac{\sqrt{r}}{2 G}\left\{\left[k-\frac{1}{2}\right] \cos \left(\frac{\theta}{2}\right)-\frac{1}{2} \cos \left(\frac{3 \theta}{2}\right)\right\}
\end{aligned}
$$

onde $\mathbf{r}$ é a distância de um ponto até a ponta da fissura , $\theta$ é ao ângulo entre o vetor posição do mesmo ponto e o eixo $\mathbf{x}$ e $\boldsymbol{K}_{\boldsymbol{I}}$ é denominado fator de intensidade de tensão. Para o estado plano de tensão o módulo de elasticidade transversal $\boldsymbol{G}$ e a variável k são expressos, em função do módulo de elasticidade $\boldsymbol{E}$ e do coeficiente de Poisson $v$, por:

$$
\begin{aligned}
& G=\frac{E}{2(1+v)} \\
& k=\frac{(3-v)}{(1+v)}
\end{aligned}
$$

Para os ensaios numéricos com esse tipo de enriquecimento, somente as bases aproximativas do domínio serão enriquecidas, correspondentes a um nó da malha de cobertura de domínio. As bases de aproximação do campo de tensões serão enriquecidas com as funções dadas pelas eq.(5.37) a eq.(5.39) e as funções interpoladoras do campo de deslocamentos no domínio com as eq.(5.40) e eq.(5.41). Assim as equações as eq.(5.1) e eq.(5.2) passam a ser escritas da seguinte forma:

- A família de funções para o campo de tensões no domínio $\Omega$ :

$$
\left.\mathfrak{I}_{N}^{2}=\left\{S_{\Omega_{j}}\right\}_{j=1}^{N} \cup\left\{S_{\Omega_{j}} \sigma_{j 1}^{\prime}\right\}_{j=1}^{N}: j=1, \ldots, N\right\}
$$

utilizada para construir a seguinte aproximação:

$$
\hat{\sigma}=\sum_{j=1}^{N} S_{\Omega_{j}}\left\{s_{\Omega_{j}}+\sigma_{j 1}^{\prime} b_{j 1}\right\}
$$


onde $s_{\Omega_{j}}$ são os graus de liberdade de tensões associadas às funções de forma originais e $\boldsymbol{b}_{\boldsymbol{j} 1}$ são os novos parâmetros nodais correspondentes a cada uma das parcelas de enriquecimento.

- A família de funções para o campo de deslocamento no domínio $\Omega$ :

$$
\Theta_{N}^{2}=\left\{\left\{U_{\Omega_{j}}\right\}_{j=1}^{N} \cup\left\{U_{\Omega_{j}} \boldsymbol{u}_{j 1}\right\}_{j=1}^{N}: j=1, \ldots, N\right\}
$$

utilizada para construir a seguinte aproximação:

$$
\hat{\boldsymbol{u}}=\sum_{j=1}^{N} U_{\Omega_{j}}\left\{\boldsymbol{u}_{\Omega_{j}}+\boldsymbol{u}_{j 1} \boldsymbol{c}_{j 1}\right\}
$$

onde $\boldsymbol{u}_{\Omega_{j}}$ são graus de liberdade em deslocamento associados às funções de forma originais e $c_{j 1}$ são os novos parâmetros nodais correspondentes a cada uma das parcelas de enriquecimento.

Nota-se que para esse tipo de enriquecimento, somente uma função de enriquecimento ( $\boldsymbol{n}_{\boldsymbol{e}}=\mathbf{1}$ ) é acrescida a um nó $\alpha$ da malha de cobertura de domínio.

Com a possibilidade de enriquecimento dos nós da malha de cobertura do domínio, as matrizes que guardam as interpolações enriquecidas do campo de tensões e deslocamentos, análogas às eq.(5.12) e eq.(5.13), podem ser escritas da seguinte forma:

$$
\begin{aligned}
S_{\Omega_{e}} & =\left[\begin{array}{llll}
\varphi_{1} \Delta_{1} & \varphi_{2} \Delta_{2} & \varphi_{3} \Delta_{3} & \varphi_{4} \Delta_{4}
\end{array}\right] \\
U_{\Omega_{e}} & =\left[\begin{array}{lllll}
\varphi_{1} \Delta_{1} & \varphi_{2} \Delta_{2} & \varphi_{3} \Delta_{3} & \varphi_{4} \Delta_{4}
\end{array}\right]
\end{aligned}
$$

onde $\Delta_{\alpha}=I_{3}+\sigma_{\alpha}^{\prime}(\alpha=1, \ldots, 4)$ para $S_{\Omega_{e}}$ e $\Delta_{\beta}=I_{2}+\boldsymbol{u}_{\beta}(\beta=1, \ldots, 4)$ para $U_{\Omega_{e}}$ são as matrizes de enriquecimento do nó $\alpha$ no domínio, sendo, ainda, $\sigma_{\alpha}$ e $\boldsymbol{u}_{\beta}$ dadas por:

$$
\sigma_{\alpha}^{\prime}=\left[\begin{array}{ccc}
\sigma_{x}^{\prime} & \mathbf{0} & \mathbf{0} \\
\mathbf{0} & \sigma_{y}^{\prime} & \mathbf{0} \\
\mathbf{0} & \mathbf{0} & \tau_{x y}^{\prime}
\end{array}\right]
$$




$$
\boldsymbol{u}_{\beta}=\left[\begin{array}{cc}
\boldsymbol{u}^{\prime} & \mathbf{0} \\
\mathbf{0} & \boldsymbol{v}^{\prime}
\end{array}\right]
$$

Nas eq.(5.50) e eq.(5.51) as componentes das matrizes são obtidas pelas expressões eq.(5.37) a eq.(5.41).

Assim, para qualquer nó $\alpha(\alpha=1, \ldots, 4)$ do elemento finito retangular $\boldsymbol{e}$, escolhido para ser enriquecido, pode-se escrever:

- Para a matriz representada pela eq.(4.11)

$$
\begin{aligned}
& S_{\Omega_{e}}^{T} f S_{\Omega_{e}}=\left\lfloor\varphi_{\alpha} \varphi_{\beta} \Delta_{\alpha}^{T} f \Delta_{\beta}\right\rfloor, \alpha=\beta=1, \ldots 4 \\
& F_{e}=\left\lfloor F_{\alpha \beta}\right\rfloor \alpha=\beta=1, \ldots 4
\end{aligned}
$$

onde:

$$
F_{\alpha \beta}=\left(\int_{\Omega_{e}} \varphi_{\alpha} \varphi_{\beta} \Delta_{\alpha}^{T} f \Delta_{\beta} d \Omega\right), \alpha, \beta=1, \ldots 4,
$$

Da mesma forma, têm-se:

- Para a matriz representada pela eq.(4.12)

$$
\begin{aligned}
& \boldsymbol{L} \boldsymbol{S}_{\Omega_{e}}=\boldsymbol{L}\left[\begin{array}{llll}
\varphi_{1} \Delta_{1} & \varphi_{2} \Delta_{2} & \varphi_{3} \Delta_{3} & \varphi_{4} \Delta_{4}
\end{array}\right]= \\
& =\left[\begin{array}{lll}
\left(L \varphi_{1}\right) \Delta_{1}+\varphi_{1}\left(L \Delta_{1}\right) & \ldots & \left(L \varphi_{4}\right) \Delta_{4}+\varphi_{4}\left(L \Delta_{4}\right)
\end{array}\right] \\
& \left(L S_{\Omega_{e}}\right)^{T}=\left[\begin{array}{c}
\Delta_{1}^{T}\left(L \varphi_{1}\right)^{T}+\varphi_{1}\left(L \Delta_{l}\right)^{T} \\
\vdots \\
\Delta_{4}^{T}\left(L \varphi_{4}\right)^{T}+\varphi_{4}\left(L \Delta_{4}\right)^{T}
\end{array}\right] \\
& \left(L S_{\Omega_{e}}\right)^{T} U_{\Omega_{e}}=\left(\Delta_{\alpha}^{T}\left(L \varphi_{\alpha}\right)^{T}+\varphi_{\alpha}\left(L \Delta_{\alpha}\right)\right)\left(\varphi_{\beta} \Delta_{\beta}\right), \alpha=\beta=1, \ldots, 4 \\
& A_{\Omega_{e}}=\left\lfloor A_{\Omega_{\alpha \beta}}\right\rfloor \alpha=\beta=1, \ldots 4
\end{aligned}
$$

onde:

$$
A_{\Omega_{\alpha \beta}}=\left(\int_{\Omega_{e}}\left(\Delta_{\alpha}^{T}\left(L \varphi_{\alpha}\right)^{T}+\varphi_{\alpha}\left(L \Delta_{\alpha}\right)\right)\left(\varphi_{\beta} \Delta_{\beta}\right) d \Omega\right), \alpha, \beta=1, \ldots 4
$$


- Para a matriz representada pela eq.(4.13)

$$
\begin{aligned}
& N S_{\Omega_{e}}=\left[\begin{array}{ll}
\left(N \varphi_{1}\right) \Delta_{1} & \left(N \varphi_{2}\right) \Delta_{2} \quad\left(N \varphi_{3}\right) \Delta_{3} \quad\left(N \varphi_{4}\right) \Delta_{4}
\end{array}\right] \\
& \left(N S_{\Omega_{e}}\right)^{T}=\left[\begin{array}{c}
\Delta_{1}^{T}\left(N \varphi_{1}\right)^{T} \\
\vdots \\
\Delta_{4}^{T}\left(N \varphi_{4}\right)^{T}
\end{array}\right] \\
& \left(N S_{\Omega_{e}}\right)^{T} U_{\Gamma_{i}}=\left(\Delta_{\alpha}^{T}\left(N \varphi_{\alpha}\right)^{T}\right)\left(\psi_{\gamma}\right), \alpha=1, \ldots, 4, \gamma=1,2 \\
& A_{\Gamma_{i}}=\left\lfloor A_{\Gamma_{\alpha \lambda}}\right\rfloor \alpha=1, \ldots 4, \gamma=1,2
\end{aligned}
$$

onde:

$$
A_{\Gamma \alpha \gamma}=\left(\int_{\Gamma_{i}}\left(\Delta_{\alpha}^{T}\left(N \varphi_{\alpha}\right)^{T}\right)\left(\psi_{\gamma}\right) d \Gamma\right), \alpha=1, . .4, \gamma=1,2,
$$

- Para a matriz representada pela eq.(4.14)

$$
\begin{aligned}
& \boldsymbol{U}_{\Gamma_{i}}^{T}=\left[\begin{array}{l}
\psi_{1} \\
\psi_{2}
\end{array}\right] \\
& \boldsymbol{Q}_{\Gamma_{i}}=\left\lfloor\boldsymbol{Q}_{\Gamma_{\gamma}}\right\rfloor \gamma=\mathbf{1 , 2}
\end{aligned}
$$

onde:

$$
Q_{\Gamma \gamma}=\left[\int_{\Gamma_{i}} \psi_{\gamma} \bar{t} d \Gamma\right], \gamma=1,2
$$

No apêndice (E) apresenta-se a expansão das matrizes $\boldsymbol{S}_{\Omega_{e}}, \boldsymbol{U}_{\Omega_{e}}, \boldsymbol{U}_{\Gamma_{i}}, \boldsymbol{F}_{e}, \boldsymbol{A}_{\Omega_{e}}$, $\boldsymbol{A}_{\Gamma_{i}}$ e $\boldsymbol{Q}_{\Gamma_{i}}$ de um elemento quadrilateral de quatro nós com o lado vertical esquerdo contido no contorno $\Gamma$, considerando-se todos os nós enriquecidos e algumas condições de enriquecimento sobre as bases aproximativas do domínio. Vale salientar que ao enriquecer diretamente as funções de forma com este tipo de função (não polinomial), os parâmetros $\boldsymbol{s}_{\Omega}$ e $\boldsymbol{u}_{\Omega}$ perdem, respectivamente, os significados de tensão nodal e deslocamento nodal, sendo que a aproximação para um certo nó deverá ser calculada ("pós-processamento") por meio das eq.(5.45) e eq.(5.47).

Quanto à integração numérica das matrizes enriquecidas com esse tipo de função, tem-se que a quadratura de Gauss torna-se insuficiente para aproximar 
exatamente a integral procurada. No trabalho de Strouboulis e Babuška e Copps (2000), onde estuda-se um problema com essa mesma característica, utilizando-se o algoritmo de DECUHR, para integração numérica das matrizes que governam o modelo analisado. Tal algoritmo é baseado em sub-divisões não uniformes do elemento finito, na direção do ponto singular.

\subsubsection{Enriquecimento Nodal com Campo de Tensões Auto-Equilibrados}

Em Bussamra (1999), cita-se o trabalho de Pereira (1993) onde foram desenvolvidos elementos finitos de tensão que utilizam bases aproximativas que geram campos de tensão auto-equilibrados. Portanto, a característica principal dessas funções é a geração de distribuição de tensões que satisfazem, a menos das forças de volume, a equação diferencial de equilíbrio. Uma forma original para a obtenção dessas funções é a utilização da função de Airy.

Considere-se a função de Airy:

$$
A(x, y)
$$

$\log 0$

$$
\begin{gathered}
\sigma_{x}=\frac{\partial^{2} A(x, y)}{\partial y^{2}} \\
\sigma_{y}=\frac{\partial^{2} A(x, y)}{\partial x^{2}} \\
\tau_{x y}=-\frac{\partial^{2} A(x, y)}{\partial x \partial y}
\end{gathered}
$$

Com $A(x, y)$ dado por:

$$
A(x, y)=\frac{1}{2} b x^{2}-c x y+\frac{1}{2} a y^{2}
$$

então

$$
\sigma=\left[\begin{array}{lll}
1 & 0 & 0 \\
0 & 1 & 0 \\
0 & 0 & 1
\end{array}\right]\left\{\begin{array}{l}
a \\
b \\
c
\end{array}\right\}
$$


Com $A(x, y)$ dado por:

$$
A(x, y)=\frac{1}{6} d x^{3}-\frac{1}{2} c x^{2} y-\frac{1}{2} b x y^{2}+\frac{1}{6} a y^{3}
$$

então

$$
\sigma=\left[\begin{array}{cccc}
y & -x & 0 & 0 \\
0 & 0 & -y & x \\
0 & y & x & 0
\end{array}\right]\left\{\begin{array}{l}
a \\
b \\
c \\
d
\end{array}\right\}
$$

Com $A(x, y)$ dado por:

$$
A(x, y)=\frac{1}{12} e x^{4}-\frac{1}{3} d x^{3} y+\frac{1}{2} c x^{2} y^{2}-\frac{1}{3} b x y^{3}+\frac{1}{12} a y^{4}
$$

então

$$
\sigma=\left[\begin{array}{ccccc}
y^{2} & -2 x y & x^{2} & 0 & 0 \\
0 & 0 & y^{2} & -2 x y & x^{2} \\
0 & y^{2} & -2 x y & x^{2} & 0
\end{array}\right]\left\{\begin{array}{l}
a \\
b \\
c \\
d \\
e
\end{array}\right\}
$$

Dessa forma, pode-se continuar com a determinação de outros campos de tensão por meio de funções de Airy de graus mais elevados. Assim, defini-se a seguinte matriz de enriquecimento com tensões auto-equilibradas atrelada a um nó $\alpha$ da malha de cobertura:

$$
A_{\alpha}=\left[\begin{array}{llllll}
E_{0} & E_{1 \alpha} & \cdots & E_{K \alpha} & \cdots & E_{d_{e} \alpha}
\end{array}\right]
$$

onde

$$
E_{0}=\left[\begin{array}{lll}
1 & 0 & 0 \\
0 & 1 & 0 \\
0 & 0 & 1
\end{array}\right]
$$




$$
\begin{aligned}
E_{1 \alpha} & =\left[\begin{array}{cccc}
y & -x & 0 & 0 \\
0 & 0 & -y & x \\
0 & y & x & 0
\end{array}\right] \\
E_{2 \alpha}= & {\left[\begin{array}{ccccc}
y^{2} & -2 x y & x^{2} & 0 & 0 \\
0 & 0 & y^{2} & -2 x y & x^{2} \\
0 & y^{2} & -2 x y & x^{2} & 0
\end{array}\right] }
\end{aligned}
$$

e $\boldsymbol{d}_{\boldsymbol{e}}$ é o grau de enriquecimento. Nota-se que

$$
n_{e}=\frac{\left(d_{e}+7\right) d_{e}}{2}
$$

onde $\boldsymbol{n}_{e}$ é o número de funções que enriquecem a base aproximativa inicial dos campos de tensão. Para os ensaios numéricos que serão realizados com o campo de tensões auto-equilibrados será utilizado no máximo $\boldsymbol{d}_{e}=2$.

Considerou-se na matriz de enriquecimento com tensões auto-equilibradas, eq.(5.78), a matriz identidade $\boldsymbol{E}_{\boldsymbol{0}}$, eq.(5.79), para que não seja destruída a característica principal do enriquecimento nodal, utilizado neste trabalho, que é a ampliação da base aproximativa inicial (PU), pela multiplicação desta por outras funções.

Observa-se que nos outros dois tipos de enriquecimento pontuados, a matriz identidade também foi inserida na matriz de enriquecimento com o objetivo de garantir que a estrutura inicial das bases aproximativas, no caso da nulidade das funções enriquecedoras, seja preservada.

Utilizando-se o enriquecimento com campo de tensões auto-equilibrados, podese definir a mesma família de funções $\mathfrak{I}_{N}^{2}$ e a aproximação $\hat{\sigma}$ para o campo de tensões no domínio.

Com a possibilidade de enriquecimento da base aproximativa do campo de tensões atrelados aos nós da malha de cobertura de domínio, utilizando o campo de tensões auto-equilibrados, a matriz de interpolação do campo de tensões dada pela eq.(4.26), pode ser escrita da seguinte forma: 


$$
S_{\Omega_{e}}=\left[\begin{array}{llll}
\varphi_{1} A_{1} & \varphi_{2} A_{2} & \varphi_{3} A_{3} & \varphi_{4} A_{4}
\end{array}\right]
$$

Observa-se da aproximação do campo de tensões no domínio, eq.(5.83), que para a matriz representada pela eq.(4.12), a matriz dada pela eq.(5.78), será anulada quando da aplicação do operador diferencial divergente $\boldsymbol{L}$ na eq.(5.83). Do exposto anteriormente, tem-se para a eq.(5.83):

$$
\begin{aligned}
& \boldsymbol{L} \boldsymbol{S}_{\Omega_{e}}=L\left[\begin{array}{llll}
\varphi_{1} A_{1} & \varphi_{2} A_{2} & \varphi_{3} A_{3} & \varphi_{4} A_{4}
\end{array}\right]= \\
& =\left[\begin{array}{llll}
\left(L \varphi_{1}\right) A_{1}+\varphi_{1}\left(L A_{1}\right) & \ldots & \left(L \varphi_{4}\right) A_{4}+\varphi_{4}\left(L A_{4}\right)
\end{array}\right]
\end{aligned}
$$

Como $\boldsymbol{A}_{1}, \ldots, \boldsymbol{A}_{4}$ são matrizes com campo de tensões auto-equilibrados

$$
\left(L A_{1}\right) \quad \ldots \quad\left(L A_{4}\right)=0
$$

e dessa forma

$$
L S_{\Omega_{e}}=\left[\begin{array}{lll}
\left(L \varphi_{1}\right) A_{1} & \ldots & \left(L \varphi_{4}\right) A_{4}
\end{array}\right]
$$

Assim, conclui-se que o enriquecimento da base aproximativa do campo de tensões da FHMT com campo de tensões auto-equlibrados preserva as três bases aproximativas envolvidas na FHMT.

Apresentar-se-á o desenvolvimento das matrizes dadas pelas eq.(4.11), eq.(4.12) e eq.(4.13), adotando-se o grau de enriquecimento $\boldsymbol{d}_{e}=\boldsymbol{1}$ e um nó (nó 4 ) do elemento finito retangular $\boldsymbol{e}$ enriquecido, considerando que o lado vertical esquerdo desse elemento pertence a malha de cobertura do contorno $\Gamma$. Ver figura 5.3

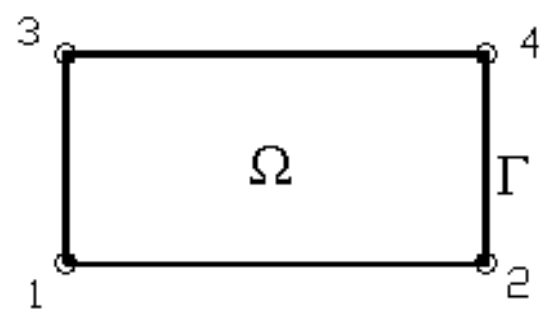

Figura 5.3 - Elemento utilizado para desenvolvimento das matrizes das eq.(4.11), eq.(4.12) e eq.(4.13)

Para esta situação de enriquecimento proposta, a eq.(5.83) toma a seguinte apresentação: 


$$
\begin{aligned}
S_{\Omega_{e}} & {\left[\begin{array}{cccccccccc}
\varphi_{1} & 0 & 0 & \varphi_{2} & 0 & 0 & \varphi_{3} & 0 & 0 & \\
0 & \varphi_{1} & 0 & 0 & \varphi_{2} & 0 & 0 & \varphi_{3} & 0 & \ldots \\
0 & 0 & \varphi_{1} & 0 & 0 & \varphi_{2} & 0 & 0 & \varphi_{3} & \\
& & & & & & & & \\
& \varphi_{4} & 0 & 0 & (y) \varphi_{4} & (-x) \varphi_{4} & 0 & 0 \\
\cdots & 0 & \varphi_{4} & 0 & 0 & 0 & (-y) \varphi_{4} & (x) \varphi_{4} \\
& 0 & 0 & \varphi_{4} & 0 & (y) \varphi_{4} & (x) \varphi_{4} & 0
\end{array}\right] }
\end{aligned}
$$

Nota-se que a dimensão da matriz $S_{\Omega_{e}}$, para essa situação de enriquecimento, é de (3 $x 16)$.

A matriz $\boldsymbol{F}_{e}$, dada pela eq.(4.11), terá uma dimensão de (16 $x$ 16), como mostra a eq.(5.88).

Linhas 1, 2 e 3 da matriz $\boldsymbol{F}_{\boldsymbol{e}}$

$\left[\begin{array}{cccccccccc}\varphi_{1} f_{11} \varphi_{1} & \varphi_{1} f_{12} \varphi_{1} & 0 & \varphi_{1} f_{11} \varphi_{2} & \varphi_{1} f_{12} \varphi_{2} & 0 & \varphi_{1} f_{11} \varphi_{3} & \varphi_{1} f_{12} \varphi_{3} & 0 \\ \varphi_{1} f_{21} \varphi_{1} & \varphi_{1} f_{22} \varphi_{1} & 0 & \varphi_{1} f_{21} \varphi_{2} & \varphi_{1} f_{22} \varphi_{2} & 0 & \varphi_{1} f_{21} \varphi_{3} & \varphi_{1} f_{22} \varphi_{3} & 0 & \ldots \\ 0 & 0 & \varphi_{1} f_{33} \varphi_{1} & 0 & 0 & \varphi_{1} f_{33} \varphi_{2} & 0 & 0 & \varphi_{1} f_{33} \varphi_{3}\end{array}\right.$

$\left.\varphi_{1} f_{11} \varphi_{4} \quad \varphi_{1} f_{12} \varphi_{4} \quad 0 \quad \varphi_{1} f_{11} \varphi_{4}(y) \quad \varphi_{1} f_{11} \varphi_{4}(-x) \quad \varphi_{1} f_{12} \varphi_{4}(-y) \varphi_{1} f_{12} \varphi_{4}(x)\right]$

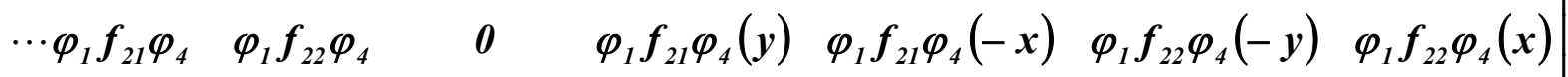
$\left.\begin{array}{lllllll}0 & 0 & \varphi_{1} f_{33} \varphi_{4} & 0 & \varphi_{1} f_{33} \varphi_{4}(y) & \varphi_{1} f_{33} \varphi_{4}(x) & 0\end{array}\right]$

Linhas 4, 5 e 6 da matriz $\boldsymbol{F}_{e}$

$\left[\begin{array}{cccccccccc}\varphi_{2} f_{11} \varphi_{1} & \varphi_{2} f_{12} \varphi_{1} & 0 & \varphi_{2} f_{11} \varphi_{2} & \varphi_{2} f_{12} \varphi_{2} & 0 & \varphi_{2} f_{11} \varphi_{3} & \varphi_{2} f_{12} \varphi_{3} & 0 \\ \varphi_{2} f_{21} \varphi_{1} & \varphi_{2} f_{22} \varphi_{1} & 0 & \varphi_{2} f_{21} \varphi_{2} & \varphi_{2} f_{22} \varphi_{2} & 0 & \varphi_{2} f_{21} \varphi_{3} & \varphi_{2} f_{22} \varphi_{3} & 0 & \ldots \\ 0 & 0 & \varphi_{2} f_{33} \varphi_{1} & 0 & 0 & \varphi_{2} f_{33} \varphi_{2} & 0 & 0 & \varphi_{2} f_{33} \varphi_{3}\end{array}\right.$

$\varphi_{2} f_{11} \varphi_{4} \quad \varphi_{2} f_{12} \varphi_{4} \quad 0 \quad \varphi_{2} f_{11} \varphi_{4}(y) \quad \varphi_{2} f_{11} \varphi_{4}(-x) \quad \varphi_{2} f_{12} \varphi_{4}(-y) \quad \varphi_{2} f_{12} \varphi_{4}(x)$

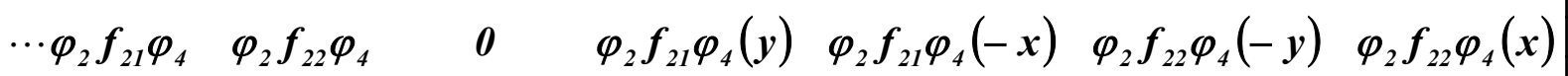
$\left.\begin{array}{lllllll}0 & 0 & \varphi_{2} f_{33} \varphi_{4} & 0 & \varphi_{2} f_{33} \varphi_{4}(y) & \varphi_{2} f_{33} \varphi_{4}(x) & 0\end{array}\right]$ 
Linhas 7, 8 e 9 da matriz $\boldsymbol{F}_{\boldsymbol{e}}$

$\left[\begin{array}{cccccccccc}\varphi_{3} f_{11} \varphi_{1} & \varphi_{3} f_{12} \varphi_{1} & 0 & \varphi_{3} f_{11} \varphi_{2} & \varphi_{3} f_{12} \varphi_{2} & 0 & \varphi_{3} f_{11} \varphi_{3} & \varphi_{3} f_{12} \varphi_{3} & 0 & \\ \varphi_{3} f_{21} \varphi_{1} & \varphi_{3} f_{22} \varphi_{1} & 0 & \varphi_{3} f_{21} \varphi_{2} & \varphi_{3} f_{22} \varphi_{2} & 0 & \varphi_{3} f_{21} \varphi_{3} & \varphi_{3} f_{22} \varphi_{3} & 0 & \ldots \\ 0 & 0 & \varphi_{3} f_{33} \varphi_{1} & 0 & 0 & \varphi_{3} f_{33} \varphi_{2} & 0 & 0 & \varphi_{3} f_{33} \varphi_{3}\end{array}\right.$

$\varphi_{3} f_{11} \varphi_{4} \quad \varphi_{3} f_{12} \varphi_{4} \quad 0 \quad \varphi_{3} f_{11} \varphi_{4}(y) \quad \varphi_{3} f_{11} \varphi_{4}(-x) \quad \varphi_{3} f_{12} \varphi_{4}(-y) \quad \varphi_{3} f_{12} \varphi_{4}(x)$ $\cdots \varphi_{3} f_{21} \varphi_{4} \quad \varphi_{3} f_{22} \varphi_{4} \quad 0 \quad \varphi_{3} f_{21} \varphi_{4}(y) \quad \varphi_{3} f_{21} \varphi_{4}(-x) \quad \varphi_{3} f_{22} \varphi_{4}(-y) \quad \varphi_{3} f_{22} \varphi_{4}(x)$ $\left.\begin{array}{lllllll}0 & 0 & \varphi_{3} f_{33} \varphi_{4} & 0 & \varphi_{3} f_{33} \varphi_{4}(y) & \varphi_{3} f_{33} \varphi_{4}(x) & 0\end{array}\right]$

Linhas 10,11 e 12 da matriz $\boldsymbol{F}_{\boldsymbol{e}}$

$\left[\begin{array}{cccccccccc}\varphi_{4} f_{11} \varphi_{1} & \varphi_{4} f_{12} \varphi_{1} & 0 & \varphi_{4} f_{11} \varphi_{2} & \varphi_{4} f_{12} \varphi_{2} & 0 & \varphi_{4} f_{11} \varphi_{3} & \varphi_{4} f_{12} \varphi_{3} & 0 & \\ \varphi_{4} f_{21} \varphi_{1} & \varphi_{4} f_{22} \varphi_{1} & 0 & \varphi_{4} f_{21} \varphi_{2} & \varphi_{4} f_{22} \varphi_{2} & 0 & \varphi_{4} f_{21} \varphi_{3} & \varphi_{4} f_{22} \varphi_{3} & 0 & \ldots \\ 0 & 0 & \varphi_{4} f_{33} \varphi_{1} & 0 & 0 & \varphi_{4} f_{33} \varphi_{2} & 0 & 0 & \varphi_{4} f_{33} \varphi_{3}\end{array}\right.$

$\varphi_{4} f_{11} \varphi_{4} \quad \varphi_{4} f_{12} \varphi_{4} \quad 0 \quad \varphi_{4} f_{11} \varphi_{4}(y) \quad \varphi_{4} f_{11} \varphi_{4}(-x) \quad \varphi_{4} f_{12} \varphi_{4}(-y) \quad \varphi_{4} f_{12} \varphi_{4}(x)$

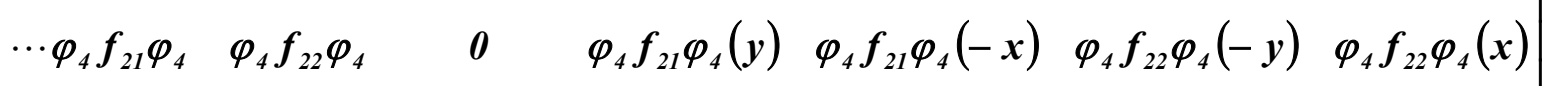
$\left.\begin{array}{lllllll}0 & 0 & \varphi_{4} f_{33} \varphi_{4} & 0 & \varphi_{4} f_{33} \varphi_{4}(y) & \varphi_{4} f_{33} \varphi_{4}(x) & 0\end{array}\right]$

Linhas $13,14,15$ e 16 da matriz $\boldsymbol{F}_{\boldsymbol{e}}$

$\begin{array}{cccccc}\varphi_{4}(y) f_{11} \varphi_{1} & \varphi_{4}(y) f_{12} \varphi_{1} & 0 & \varphi_{4}(y) f_{11} \varphi_{2} & \varphi_{4}(y) f_{11} \varphi_{2} & 0 \\ \varphi_{4}(-x) f_{11} \varphi_{1} & \varphi_{4}(-x) f_{12} \varphi_{1} & \varphi_{4}(y) f_{33} \varphi_{1} & \varphi_{4}(-x) f_{11} \varphi_{2} & \varphi_{4}(-x) f_{12} \varphi_{2} & \varphi_{4}(y) f_{33} \varphi_{2} \ldots \\ \varphi_{4}(-y) f_{21} \varphi_{1} & \varphi_{4}(-y) f_{22} \varphi_{1} & \varphi_{4}(x) f_{33} \varphi_{1} & \varphi_{4}(-y) f_{21} \varphi_{2} & \varphi_{4}(-y) f_{22} \varphi_{2} & \varphi_{4}(x) f_{33} \varphi_{2} \\ \varphi_{4}(x) f_{21} \varphi_{1} & \varphi_{4}(x) f_{22} \varphi_{1} & 0 & \varphi_{4}(x) f_{21} \varphi_{2} & \varphi_{4}(x) f_{22} \varphi_{2} & 0 \\ & & & & & \\ \varphi_{4}(y) f_{11} \varphi_{3} & \varphi_{4}(y) f_{12} \varphi_{3} & 0 & \varphi_{4}(y) f_{11} \varphi_{4} & \varphi_{4}(y) f_{11} \varphi_{4} & 0 \\ \varphi_{4}(-x) f_{11} \varphi_{3} & \varphi_{4}(-x) f_{12} \varphi_{3} & \varphi_{4}(y) f_{33} \varphi_{3} & \varphi_{4}(-x) f_{11} \varphi_{4} & \varphi_{4}(-x) f_{12} \varphi_{4} & \varphi_{4}(y) f_{33} \varphi_{4} \ldots \\ \varphi_{4}(-y) f_{21} \varphi_{3} & \varphi_{4}(-y) f_{22} \varphi_{3} & \varphi_{4}(x) f_{33} \varphi_{3} & \varphi_{4}(-y) f_{21} \varphi_{4} & \varphi_{4}(-y) f_{22} \varphi_{4} & \varphi_{4}(x) f_{33} \varphi_{4} \\ \varphi_{4}(x) f_{21} \varphi_{3} & \varphi_{4}(x) f_{22} \varphi_{3} & 0 & \varphi_{4}(x) f_{21} \varphi_{4} & \varphi_{4}(x) f_{22} \varphi_{4} & 0\end{array}$




$$
\left.\begin{array}{cccc}
\varphi_{4}(y) f_{11} \varphi_{4}(y) & \varphi_{4}(-y) f_{11} \varphi_{4}(x) & \varphi_{4}(-y) f_{12} \varphi_{4}(y) & \varphi_{4}(y) f_{12} \varphi_{4}(x) \\
\varphi_{4}(-x) f_{11} \varphi_{4}(y) & \varphi_{4}(x) f_{11} \varphi_{4}(x)+\varphi_{4}(y) f_{33} \varphi_{4}(y) & \varphi_{4}(x) f_{12} \varphi_{4}(y)+\varphi_{4}(y) f_{33} \varphi_{4}(x) & \varphi_{4}(-x) f_{12} \varphi_{4}(x) \\
\varphi_{4}(-y) f_{21} \varphi_{4}(y) & \varphi_{4}(y) f_{21} \varphi_{4}(x)+\varphi_{4}(x) f_{33} \varphi_{4}(y) & \varphi_{4}(y) f_{22} \varphi_{4}(y)+\varphi_{4}(x) f_{33} \varphi_{4}(x) & \varphi_{4}(-y) f_{22} \varphi_{4}(x) \\
\varphi_{4}(x) f_{21} \varphi_{4}(y) & \varphi_{4}(-x) f_{21} \varphi_{4}(x) & \varphi_{4}(-x) f_{22} \varphi_{4}(y) & \varphi_{4}(x) f_{22} \varphi_{4}(x)
\end{array}\right]
$$

Onde $\boldsymbol{f}_{11}, \boldsymbol{f}_{12}, \boldsymbol{f}_{21}, \boldsymbol{f}_{22}$ e $\boldsymbol{f}_{33}$ são os termos da matriz de flexibilidade considerada na análise do problema.

A matriz $\boldsymbol{A}_{\Omega_{e}}$, dada pela eq.(4.12), para essa situação de enriquecimento, é de (16 $x$ 8), como mostra a eq.(5.89). Vale lembrar que no desenvolvimento da matriz $\boldsymbol{A}_{\Omega_{e}}$ para esse tipo de enriquecimento $\boldsymbol{L} \boldsymbol{A}_{\alpha}=\boldsymbol{0}$.

Linhas 1,2 e 3 da matriz $\boldsymbol{A}_{\Omega_{e}}$

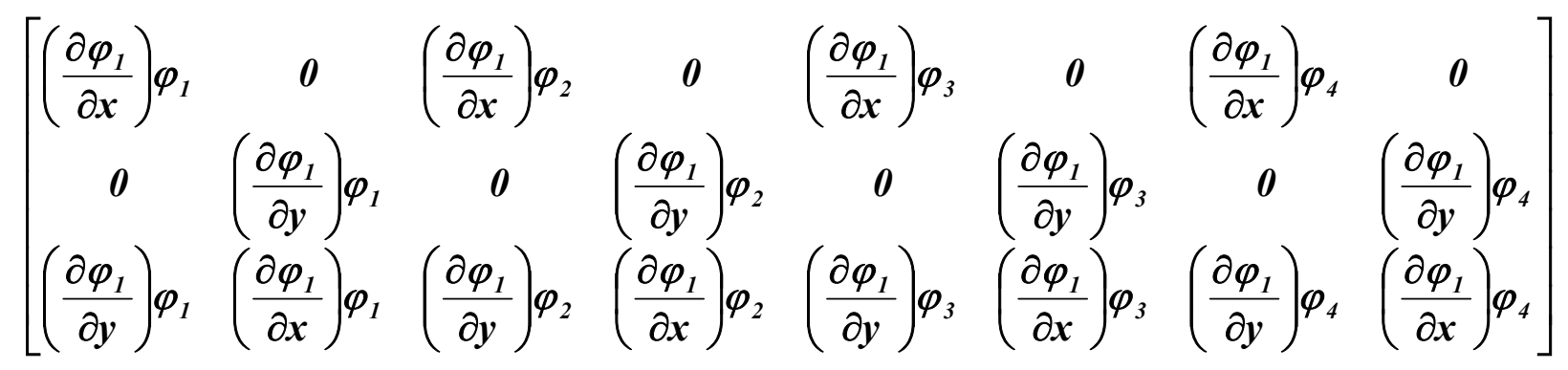

Linhas 4, 5 e 6 da matriz $\boldsymbol{A}_{\Omega_{e}}$

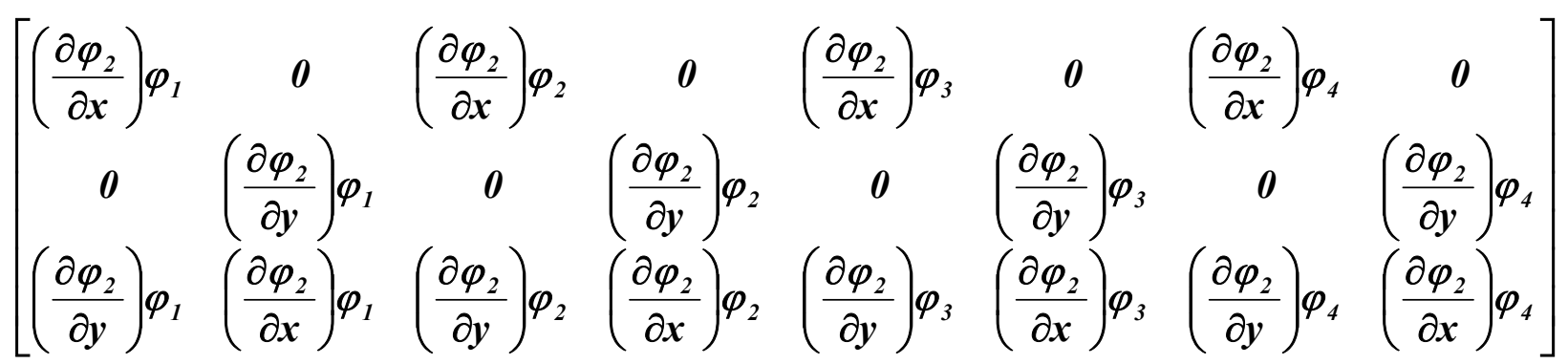


Linhas 7,8 e 9 da matriz $\boldsymbol{A}_{\Omega_{e}}$

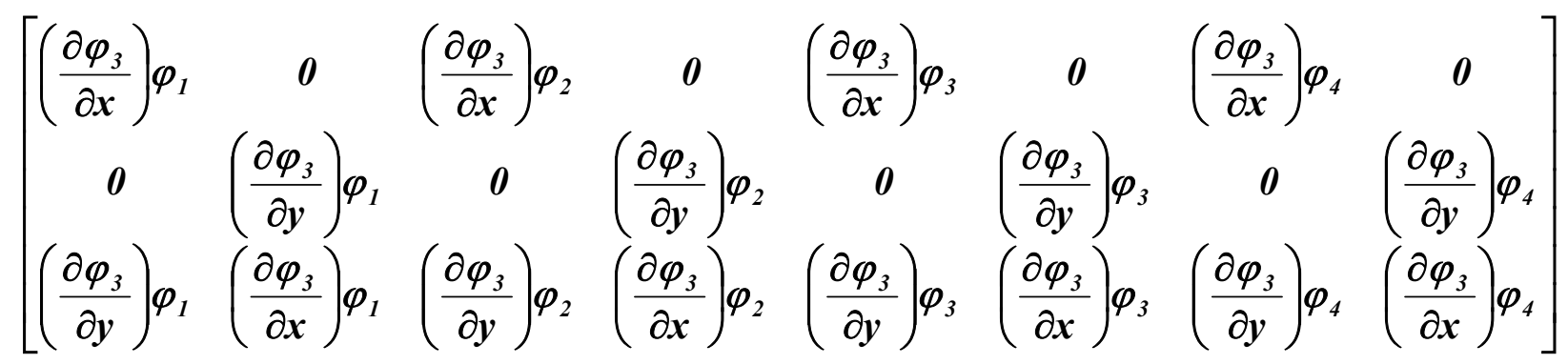

Linhas 10,11 e 12 da matriz $\boldsymbol{A}_{\Omega_{e}}$

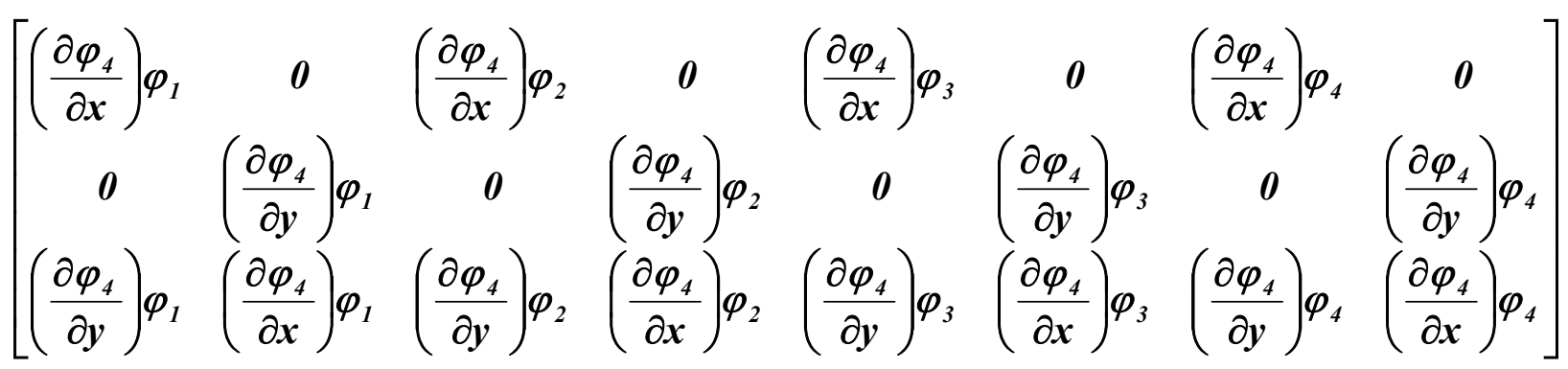

Linhas $13,14,15$ e 16 da matriz $\boldsymbol{A}_{\Omega e}$

$$
\left[\begin{array}{cc}
\left(\frac{\partial \varphi_{4}}{\partial x}\right)(y) \varphi_{1} & 0 \\
\left(\frac{\partial \varphi_{4}}{\partial x}\right)(-x) \varphi_{1}+\left(\frac{\partial \varphi_{4}}{\partial y}\right)(y) \varphi_{1} & \left(\frac{\partial \varphi_{4}}{\partial x}\right)(y) \varphi_{1} \\
\left(\frac{\partial \varphi_{4}}{\partial y}\right)(x) \varphi_{1} & \left(\frac{\partial \varphi_{4}}{\partial y}\right)(-y) \varphi_{1}+\left(\frac{\partial \varphi_{4}}{\partial x}\right)(x) \varphi_{1} \\
0 & \left(\frac{\partial \varphi_{4}}{\partial y}\right)(x) \varphi_{1} \\
\left(\frac{\partial \varphi_{4}}{\partial x}\right)(-x) \varphi_{2}+\left(\frac{\partial \varphi_{4}}{\partial y}\right)(y) \varphi_{2} & \left(\frac{\partial \varphi_{4}}{\partial x}\right)(y) \varphi_{2} \\
\left(\frac{\partial \varphi_{4}}{\partial y}\right)(x) \varphi_{2} & \left(\frac{\partial \varphi_{4}}{\partial y}\right)(-y) \varphi_{2}+\left(\frac{\partial \varphi_{4}}{\partial x}\right)(x) \varphi_{2}
\end{array}\right]
$$




$$
\begin{aligned}
& \left(\frac{\partial \varphi_{4}}{\partial x}\right)(y) \varphi_{3} \\
& \left(\frac{\partial \varphi_{4}}{\partial x}\right)(-x) \varphi_{3}+\left(\frac{\partial \varphi_{4}}{\partial y}\right)(y) \varphi_{3} \quad\left(\frac{\partial \varphi_{4}}{\partial x}\right)(y) \varphi_{3} \\
& \left(\frac{\partial \varphi_{4}}{\partial y}\right)(x) \varphi_{3} \quad\left(\frac{\partial \varphi_{4}}{\partial y}\right)(-y) \varphi_{3}+\left(\frac{\partial \varphi_{4}}{\partial x}\right)(x) \varphi_{3} \\
& \text { o }\left(\frac{\partial \varphi_{4}}{\partial y}\right)(x) \varphi_{3}
\end{aligned}
$$

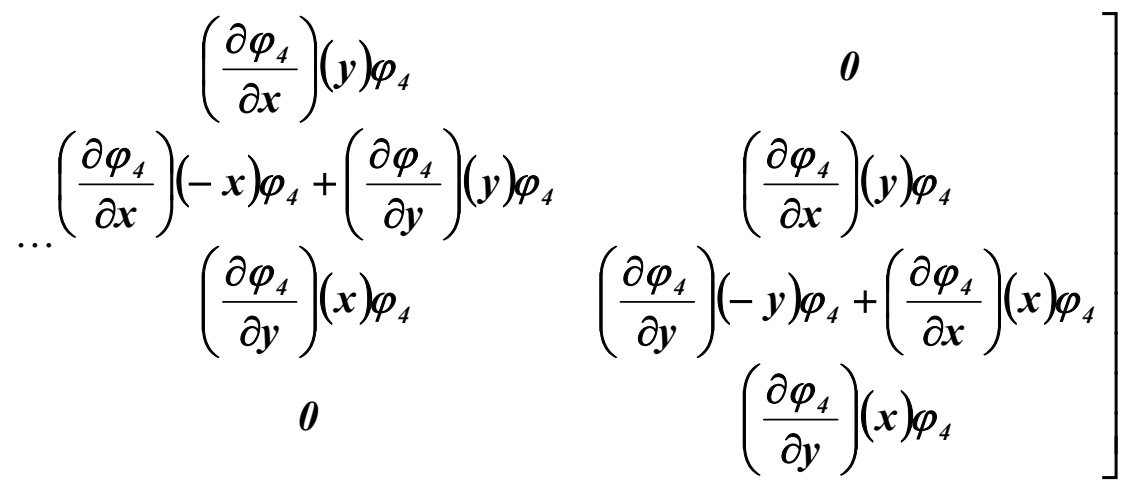

A matriz $\boldsymbol{A}_{\Gamma_{i}}$ dada pela eq.(4.13) terá uma dimensão de (16 x 4), como mostra a eq.(5.90).

Linhas 1,2 e 3 da matriz $\boldsymbol{A}_{\Gamma_{i}}$

$$
\left[\begin{array}{cccc}
n_{x} \varphi_{1} \psi_{1} & 0 & n_{x} \varphi_{1} \psi_{2} & 0 \\
0 & n_{y} \varphi_{1} \psi_{1} & 0 & n_{y} \varphi_{1} \psi_{2} \\
n_{y} \varphi_{1} \psi_{1} & n_{x} \varphi_{1} \psi_{1} & n_{y} \varphi_{1} \psi_{2} & n_{x} \varphi_{1} \psi_{2}
\end{array}\right]
$$

Linhas 4, 5 e 6 da matriz $\boldsymbol{A}_{\Gamma_{i}}$

$$
\left[\begin{array}{cccc}
n_{x} \varphi_{2} \psi_{1} & 0 & n_{x} \varphi_{2} \psi_{2} & 0 \\
0 & n_{y} \varphi_{2} \psi_{1} & 0 & n_{y} \varphi_{2} \psi_{2} \\
n_{y} \varphi_{2} \psi_{1} & n_{x} \varphi_{2} \psi_{1} & n_{y} \varphi_{2} \psi_{2} & n_{x} \varphi_{2} \psi_{2}
\end{array}\right]
$$


Linhas 7, 8 e 9 da matriz $\boldsymbol{A}_{\Gamma_{i}}$

$$
\left[\begin{array}{cccc}
n_{x} \varphi_{3} \psi_{1} & 0 & n_{x} \varphi_{3} \psi_{2} & 0 \\
0 & n_{y} \varphi_{3} \psi_{1} & 0 & n_{y} \varphi_{3} \psi_{2} \\
n_{y} \varphi_{3} \psi_{1} & n_{x} \varphi_{3} \psi_{1} & n_{y} \varphi_{3} \psi_{2} & n_{x} \varphi_{3} \psi_{2}
\end{array}\right]
$$

Linhas 10, 11 e 12 da matriz $\boldsymbol{A}_{\Gamma_{i}}$

$$
\left[\begin{array}{cccc}
n_{x} \varphi_{4} \psi_{1} & 0 & n_{x} \varphi_{4} \psi_{2} & 0 \\
0 & n_{y} \varphi_{4} \psi_{1} & 0 & n_{y} \varphi_{4} \psi_{2} \\
n_{y} \varphi_{4} \psi_{1} & n_{x} \varphi_{4} \psi_{1} & n_{y} \varphi_{4} \psi_{2} & n_{x} \varphi_{4} \psi_{2}
\end{array}\right]
$$

Linhas 13, 14, 15 e 16 da matriz $\boldsymbol{A}_{\Gamma_{i}}$

$$
\begin{aligned}
& {\left[\begin{array}{cc}
n_{x} \varphi_{4}(y) \psi_{1} & 0 \\
n_{x} \varphi_{4}(-x) \psi_{1}+n_{y} \varphi_{4}(y) \psi_{1} & n_{x} \varphi_{4}(y) \psi_{1} \\
n_{y} \varphi_{4}(x) \psi_{1} & n_{y} \varphi_{4}(-y) \psi_{1}+n_{x} \varphi_{4}(x) \psi_{1}{ }^{\cdots} \\
0 & n_{y} \varphi_{4}(x) \psi_{1}
\end{array}\right.} \\
& \left.\begin{array}{cc}
\boldsymbol{n}_{x} \varphi_{4}(y) \psi_{2} & 0 \\
\boldsymbol{n}_{x} \varphi_{4}(-x) \psi_{2}+n_{y} \varphi_{4}(y) \psi_{2} & n_{x} \varphi_{4}(y) \psi_{2} \\
n_{y} \varphi_{4}(x) \psi_{2} & n_{y} \varphi_{4}(-y) \psi_{2}+n_{x} \varphi_{4}(x) \psi_{2} \\
0 & n_{y} \varphi_{4}(x) \psi_{2}
\end{array}\right]
\end{aligned}
$$

onde $\boldsymbol{n}_{\boldsymbol{x}}$ e $\boldsymbol{n}_{\boldsymbol{y}}$ são as normais ao contorno $\Gamma$ do elemento.

A matriz $\boldsymbol{Q}_{\Gamma_{i}}$ dada pela eq.(4.14) terá uma dimensão de (4 x 1), ou seja, não terá sua dimensão alterada com esse tipo de enriquecimento.

No tocante aos pontos de Gauss necessários para integração das matrizes enriquecidas de cada elemento, foram adotados os mesmos procedimentos utilizados quando do enriquecimento polinomial.

\subsubsection{Enriquecimento com Funções Trigonométricas}

Para o enriquecimento das bases aproximativas da FHMT com funções trigonométricas e o desenvolvimento das novas famílias de funções com suas 
respectivas aproximações, utilizou-se o mesmo procedimento quando do enriquecimento nodal polinomial.

Como funções trigonométricas enriquecedoras, dos campos envolvidos na FHMT, foram utilizadas $\operatorname{sen}\left[\left(\boldsymbol{Y}-\boldsymbol{Y}_{\alpha}\right)^{2}\right]$ e $\cos \left[\left(\boldsymbol{X}-\boldsymbol{X}_{\alpha}\right)^{2}\right\rfloor$ onde $\boldsymbol{X}_{\alpha}, \boldsymbol{Y}_{\alpha}$ são as coordenadas adimensionais. Para esse tipo de enriquecimento, somente a função $\operatorname{sen}\left\lfloor\left(\boldsymbol{Y}-\boldsymbol{Y}_{\alpha}\right)^{2}\right\rfloor$ é nula no nó enriquecido ("função bolha") . O enriquecimento das funções de forma com $\cos \left[\left(X-X_{\alpha}\right)^{2}\right\rfloor$ não gera a "função bolha" e os parâmetros nodais de tensão e deslocamento perdem, respectivamente o significado de tensão e deslocamento nodal e a aproximação para um certo nó deverá ser "pós-processada", como foi feita para o enriquecimento com soluções da Mecânica da Fratura Elástica Linear .

Constatou-se que são necessários no mínimo cinco pontos de Gauss em cada direção do elemento de domínio e três pontos de Gauss no elemento de contorno para integração das matrizes enriquecidas de cada elemento com esse tipo de função enriquecedora. 


\section{Estudo Inicial das Condições de Convergência da FHMT com Enriquecimento Nodal}

\subsection{Introdução}

Neste capítulo são discutidas questões relativas à convergência das soluções obtidas da FHMT com enriquecimento nodal. Apresenta-se, uma generalização do Teste do Mosaico ("Patch Test") para Formulações Mistas ("The Patch Test For Mixed Formulations" proposto no trabalho de Zienkiewicz et al. (1986)) como ponto inicial de análise das condições gerais para solvibilidade e convergência do sistema de equações lineares que governa a FHMT com enriquecimento nodal.

Focando-se ainda a convergência, será estudada posteriormente a condição de Babuška-Brezzi (inf-sup) que é necessária e suficiente para estabilidade de qualquer formulação desenvolvida com a técnica do MEF.

\subsection{O Teste do Mosaico}

\subsubsection{Considerações Iniciais}

Para que se garanta a convergência da solução aproximativa de qualquer formulação do MEF com o refinamento da malha, o espaço da aproximação escolhida deve satisfazer basicamente duas condições: a de consistência e a de estabilidade.

Uma das garantias oferecidas pela condição de consistência é que com o tamanho dos elementos finitos tendendo a zero, a aproximação adotada passa a representar exatamente, no limite, a solução da equação diferencial e as condições de contorno do problema. Um outro aspecto resultante da condição de consistência é a compatibilidade. A compatibilidade exige que as funções de forma utilizadas na aproximação dos campos devem ser tais que garantam a continuidade destes. 
Já se a condição de estabilidade é satisfeita, a solução do sistema de equações discretas existe e converge, com o refinamento, para uma solução única. Tendo-se em vista um elemento ou uma malha de elementos finitos, a condição de estabilidade pressupõe o atendimento a duas outras condições: a primeira é representada pela elipsidade e a segunda pela condição de Babuška-Brezzi (inf-sup), proposta independentemente por Babuška $(1973,1977)$ e Brezzi (1974).

Neste item a ênfase é dada à estabilidade. Como primeiro passo no estudo das condições para a convergência da solução da FHMT com enriquecimento nodal, adaptam-se as técnicas definidas no trabalho de Zienkiewicz et al. (1986). Naquele trabalho representa-se uma condição algébrica simples para se garantir a estabilidade de formulações mistas.

Verifica-se que esta condição está ligada à solvibilidade do sistema de equações lineares discretas da formulação Mista, e constitui-se numa condição algébrica necessária para a não singularidade desse sistema linear.

\subsubsection{O Teste do Mosaico}

No Teste do Mosaico para Formulações Mistas, apresentado no trabalho de Zienkiewicz et al. (1986), analisa-se um sistema típico resultante da aproximação mista para dois campos, dado por:

$$
\left[\begin{array}{cc}
A & B \\
B^{T} & 0
\end{array}\right]\left\{\begin{array}{l}
x \\
y
\end{array}\right\}=\left\{\begin{array}{l}
f_{1} \\
f_{2}
\end{array}\right\}
$$

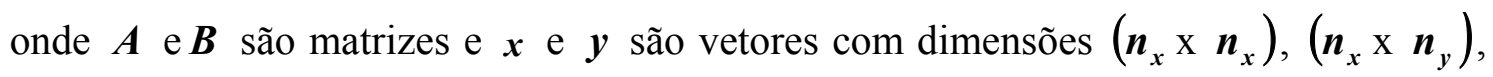
$\left(\boldsymbol{n}_{\boldsymbol{x}}\right)$ e $\left(\boldsymbol{n}_{\boldsymbol{y}}\right)$ respectivamente. $\left(\boldsymbol{n}_{\boldsymbol{x}}\right)$ e $\left(\boldsymbol{n}_{\boldsymbol{y}}\right)$ são os números de incógnitas das variáveis $\boldsymbol{x}$ e $\boldsymbol{y}$, respectivamente.

Os vetores $\boldsymbol{x}$ e $\boldsymbol{y}$ podem representar, por exemplo, os campos de tensão e deslocamento $(\sigma$ e $\boldsymbol{u})$ e assim a eq. (6.1) resulta da aproximação do funcional de Reissner-Helinger dado pela eq.(2.29).

A condição algébrica necessária para que o sistema de equações dado por eq.(6.1), seja não-singular é:

$$
\boldsymbol{n}_{\boldsymbol{x}} \geq \boldsymbol{n}_{\boldsymbol{y}}
$$


ou seja, o número de graus de liberdade em tensão deve ser maior ou igual ao número de graus de liberdade em deslocamento.

Observa-se que na eq.(6.1), se o número de varáveis do vetor $\boldsymbol{y}\left(\boldsymbol{n}_{\boldsymbol{y}}\right)$ for superior ao número de variáveis do vetor $\boldsymbol{x}\left(\boldsymbol{n}_{\boldsymbol{x}}\right)$, pode-se obter um vetor $\boldsymbol{x}$ diretamente da segunda linha desse sistema, conduzindo a múltiplas soluções para o vetor $\boldsymbol{y}$ e comprometendo, naturalmente, a convergência.

A condição representada pela eq.(6.2) fica evidente com o seguinte desenvolvimento.

Assume-se que na eq.(6.1) a matriz $\boldsymbol{A}$ seja não-singular. Assim, pode-se eliminar o vetor $\boldsymbol{x}$ da primeira linha e substituí-la na segunda para obter:

$$
\left(B^{T} A^{-1} B\right) y=-f_{2}+B^{T} A^{-1} f_{1}
$$

Para a determinação do vetor $\boldsymbol{y}$ é necessário garantir que o produto matricial $\left(\boldsymbol{B}^{T} \boldsymbol{A}^{-1} \boldsymbol{B}\right)$ seja inversível. Então:

$$
\underbrace{\boldsymbol{B}_{\left(n_{y} \times n_{x}\right)}^{T} \boldsymbol{A}_{\left(n_{x} \times n_{x}\right)}^{-1}}_{(I)} \underbrace{\boldsymbol{B}_{\left(n_{x} \times n_{y}\right)}}_{(I I)}
$$

Da primeira multiplicação matricial da eq.(6.4), representada por $(I)$, resultará a seguinte matriz:

$$
C_{\left(n_{y} \mathrm{x} n_{x}\right)}
$$

Agora, multiplicando-se a eq.(6.5) por (II), tem-se:

$$
\boldsymbol{C}_{\left(n_{y} \times n_{x}\right)} \boldsymbol{B}_{\left(n_{x} \times n_{y}\right)}=\boldsymbol{D}_{\left(n_{y} \times n_{y}\right)}
$$

Da Álgebra Matricial, tem-se a seguinte condição: dadas uma matriz $\boldsymbol{C}$ de dimensão $\left(\boldsymbol{n}_{\boldsymbol{y}} \mathrm{x} \boldsymbol{n}_{\boldsymbol{x}}\right)$ e uma matriz $\boldsymbol{B}$ de dimensão $\left(\boldsymbol{n}_{\boldsymbol{x}} \mathrm{x} \boldsymbol{n}_{\boldsymbol{y}}\right)$, a matriz $\boldsymbol{D}$ resultante da multiplicação de $\boldsymbol{C B}$ só será inversível se $\boldsymbol{n}_{\boldsymbol{x}} \geq \boldsymbol{n}_{\boldsymbol{y}}$. A prova dessa condição é desenvolvida no apêndice (C).

A condição dada pela eq.(6.2), ao evitar infinitas soluções para o vetor $\boldsymbol{y}$, garante ainda que o vetor nulo não será uma solução para o vetor $\boldsymbol{x}$. Vale salientar que 
a eq.(6.2) é uma condição necessária, mas não suficiente para garantir a não singularidade da matriz $\boldsymbol{D}$. Para que exista solução única do vetor $\boldsymbol{y}$, deve-se ainda ter:

$$
B \boldsymbol{B} \neq \boldsymbol{0} \quad \forall \quad \boldsymbol{y} \neq \boldsymbol{0}
$$

Os autores ainda afirmam que todas as condições apresentadas anteriormente, de certa forma, estão presentes na condição de Babuška-Brezzi.

Em termos práticos, o Teste do Mosaico para Formulações Mistas é realizado basicamente pela verificação da condição dada pela eq.(6.2) em "mosaicos" de elementos na discretização estudada. É importante salientar que todos os "mosaicos" possíveis devem ser investigados e a falha em qualquer um deles pode comprometer a solução global, mesmo quando um comportamento não-singular para todo o conjunto é obtido.

Para estender os conceitos fundamentais do trabalho de Zienkiewicz et al. (1986) para a FHMT com enriquecimento nodal, considere-se o sistema de equações dado pela eq.(3.32) expresso da seguinte forma:

$$
\left\{\begin{array}{l}
F s_{\Omega}+A_{\Omega} q_{\Omega}-A_{\Gamma} q_{\Gamma}=0 \\
A_{\Omega}^{T} s_{\Omega}=0 \\
-A_{\Gamma}^{T} s_{\Omega}=-Q_{\Gamma}
\end{array}\right.
$$

Como primeira condição algébrica necessária para existência de solução única da eq.(6.8), tem-se:

$$
s_{\Omega} \geq \boldsymbol{q}_{\Omega}+\boldsymbol{q}_{\Gamma}
$$

pois de forma similar a proposta do trabalho de Zienkiewicz et al. (1986), se $\boldsymbol{s}_{\Omega}<\boldsymbol{q}_{\Omega}+\boldsymbol{q}_{\Gamma}$, a segunda e terceira equações da eq.(6.8) poderiam ser utilizadas para determinação de $s_{\Omega}$ o que conduziria múltiplas soluções para os vetores $\boldsymbol{q}_{\Omega}$ e $\boldsymbol{q}_{\Gamma}$.

Sabe-se que a dimensão da matriz $\boldsymbol{F}$, como já comentado, depende da dimensão da matriz $S_{\Omega}$, que coleta as aproximações do campo de tensão, e da matriz de flexibilidade $\boldsymbol{f}$. Assim, a matriz $\boldsymbol{F}$ possui a seguinte dimensão:

$$
F_{\left(s_{\Omega} \times s_{\Omega}\right)}
$$


Da mesma forma, pode-se definir para as matrizes $\boldsymbol{A}_{\Omega}$ e $\boldsymbol{A}_{\Gamma}$ as seguintes dimensões respectivamente:

$$
\mathrm{e} \quad \begin{aligned}
& \boldsymbol{A}_{\Omega\left(s_{\Omega} \times q_{\Omega}\right)} \\
& \boldsymbol{A}_{\Gamma\left(s_{\Omega} \times q_{\Gamma}\right)}
\end{aligned}
$$

Além disso, a matriz $\boldsymbol{F}$ é simétrica e com a utilização de funções linearmente independentes para aproximação do campo de tensão, tem-se que $\boldsymbol{F}$ também é inversível. Então, pode-se eliminar $\boldsymbol{s}_{\Omega}$ da primeira equação do sistema (6.8) e substituila na segunda e terceira equação do mesmo. Com isso, escreve-se o seguinte sistema:

$$
\left\{\begin{array}{l}
\underbrace{\left(-A_{\Omega}^{T} F^{-1} A_{\Omega}\right)}_{(I)} q_{\Omega}+\underbrace{\left(A_{\Omega}^{T} F^{-1} A_{\Gamma}\right)}_{(I I)} q_{\Gamma}=0 \\
\underbrace{\left(-A_{\Gamma}^{T} F^{-1} A_{\Omega}\right)}_{(I I I)} q_{\Omega}+\underbrace{\left(A_{\Gamma}^{T} F^{-1} A_{\Gamma}\right)}_{(I V)} q_{\Gamma}=-Q_{\Gamma}
\end{array}\right.
$$

No sistema (6.13), os produtos matriciais representados por $(\boldsymbol{I I})$ e (III) não são quadrados e por isso também não são inversíveis. Para a obtenção de $\boldsymbol{q}_{\Omega}$ e $\boldsymbol{q}_{\Gamma}$ obrigatoriamente, deve-se garantir que os produtos matriciais representados por $(I)$ e $(\boldsymbol{I V})$ gerem matrizes inversíveis. Para que isso ocorra, tem-se necessariamente:

$$
(I I)=\left(A_{\Omega}^{T} F^{-1} A_{\Gamma}\right) \Rightarrow(\underbrace{A_{\Omega\left(q_{\Omega} \times \Omega_{\Omega}\right)}^{T} F_{\left(s_{\Omega} \times s_{\Omega}\right)}}_{C_{\left(q_{\Omega} \times s_{\Omega}\right)}}{ }^{-1} A_{\Gamma\left(s_{\Omega} \times q_{\Omega}\right)}) \Rightarrow \underbrace{C_{\left(q_{\Omega} \times s_{\Omega}\right)} A_{\Gamma\left(s_{\Omega} \times q_{\Omega}\right)}}_{D_{\left(q_{\Omega} \times q_{\Omega}\right)}}
$$

A matriz $\boldsymbol{D}_{\left(q_{\Omega} \times q_{\Omega}\right)}$ da eq.(6.14) só terá inversa se:

$$
s_{\Omega} \geq \boldsymbol{q}_{\Omega}
$$

Analogamente,

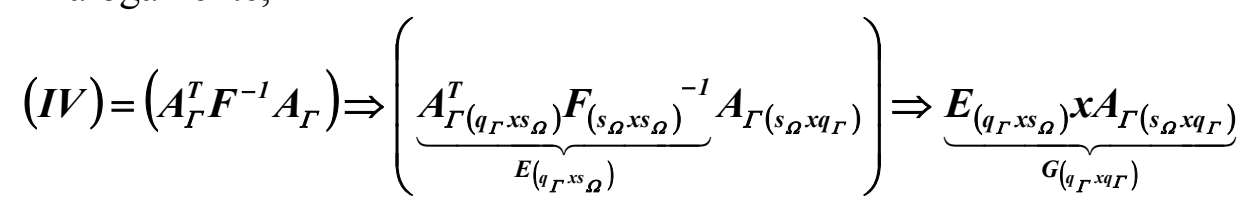

e, da mesma forma, a matriz $\boldsymbol{D}_{\left(q_{\Gamma} \times q_{\Gamma}\right)}$ da eq.(6.16) só terá inversa se:

$$
s_{\Omega} \geq q_{\Gamma}
$$


A condição fornecida pela eq.(6.15) está ligada à eq.(6.9), pois se o Teste do Mosaico for aplicado a um único elemento finito no domínio da estrutura analisada, sem conectividade com o contorno da mesma, a eq, (6.9) assumirá a mesma forma da eq.(6.15). A eq.(6.14) também está embutida na eq.(6.9), pois quando esta é aplicada aos elementos da malha de contorno, também existirá a igualdade entre as equações eq.(6.9) e eq.(6.17). Então, se a eq.(6.15) é satisfeita no domínio e a eq.(6.17) no contorno, tem-se que a eq.(6.9) também é satisfeita.

Assim, o Teste do Mosaico para FHMT com enriquecimento nodal pode ser aplicado da mesma forma que o Teste do Mosaico para Formulações Mistas. Escolhidos os vários "mosaicos" de elementos do problema, investigam-se as condições eq.(6.15) e eq.(6.17) para a malha de domínio e contorno respectivamente. Vale ressaltar que esse teste é uma condição necessária, mas não suficiente para garantia da estabilidade da solução da eq.(3.32). 


\section{Exemplos Numéricos}

\subsection{Introdução}

Os exemplos propostos consistem de duas chapas retangulares submetidas a condições distintas de carregamento e vinculação. Como já enfatizado, no trabalho, as análises pressupõem um regime de comportamento elástico-linear. Por simplificação não foram adotadas unidades para os parâmetros elásticos e dimensões nos dois problemas. Consideram-se o módulo de Young e coeficiente de Poisson, respectivamente, com os seguintes valores: $\boldsymbol{E}=\mathbf{1 0 0 0}$ e $\boldsymbol{v}=\mathbf{0 , 3}$.

As duas chapas retangulares, representadas nas figuras 7.1 e 7.3 , possuem as mesmas dimensões $\mathbf{6} \boldsymbol{a} \times \mathbf{3} \boldsymbol{a}$ (adotou-se $\boldsymbol{a}=\mathbf{2 0}$ unidades de comprimento).

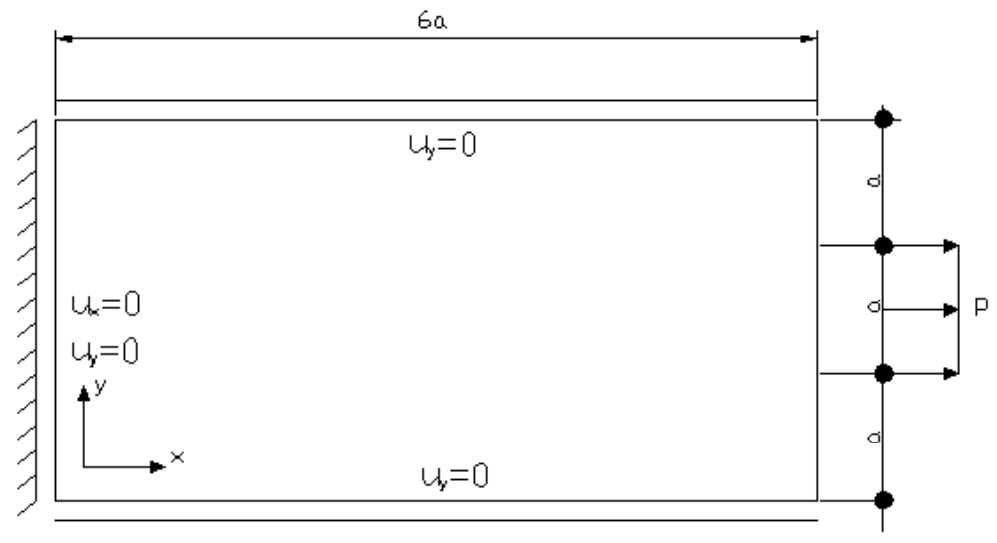

Figura 7.1 - Chapa tracionada simetricamente.

A chapa da figura 7.1, que dá origem ao problema 1, deve apresentar uma distribuição de tensões bastante regular esperando-se, apenas, uma maior concentração de tensões próximo da aplicação do carregamento. Como condição de contorno essenciais desse problema, têm-se que o lado vertical esquerdo é fixo $\left(\boldsymbol{u}_{\boldsymbol{x}}=\boldsymbol{0}\right.$ e $\left.\boldsymbol{u}_{\boldsymbol{y}}=\boldsymbol{0}\right)$; já os lados horizontal superior e inferior possuem o deslocamento na direção $\boldsymbol{y}$ 
impedido $\left(\boldsymbol{u}_{\boldsymbol{y}}=\boldsymbol{0}\right)$. Já a figura 7.3 que mostra o problema 2, decorre da exploração da dupla simetria de uma chapa com fenda central (ver figura 7.2). O problema 2, além da descontinuidade associada à força aplicada, apresenta um ponto de singularidade forte na extremidade da fenda e por essa razão a estimativa para os campos de tensão naquela região é de difícil simulação. Ambas as chapas são tracionadas por $p=10$ unidades de força distribuída por unidade de comprimento.

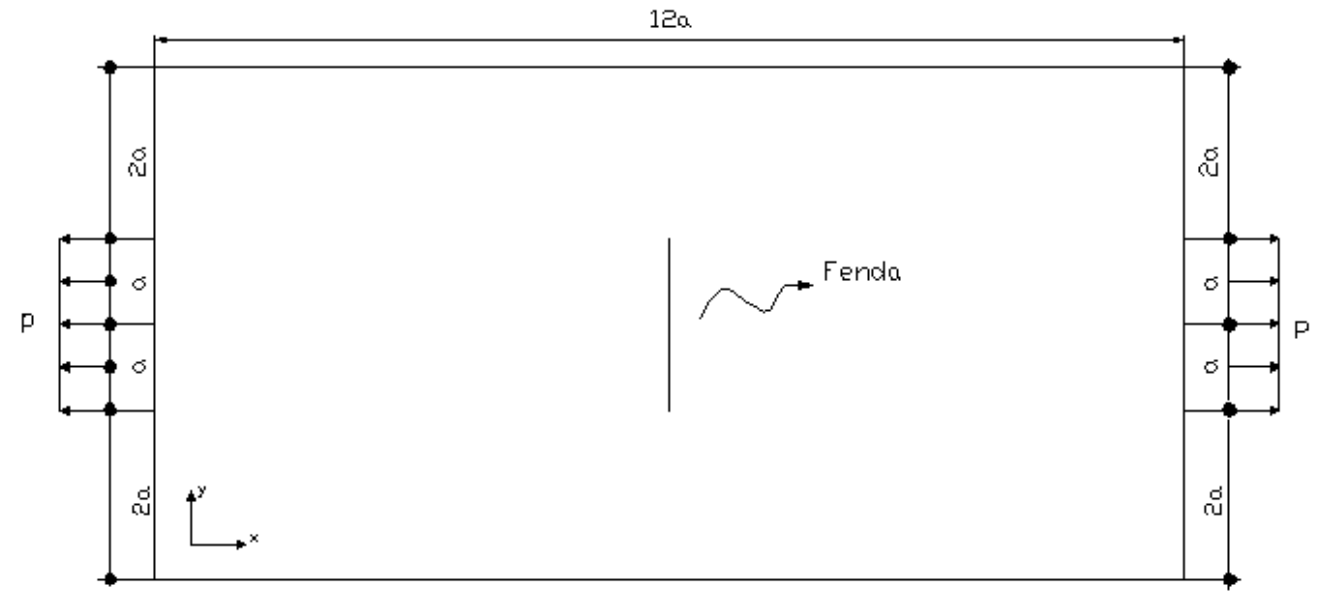

Figura 7.2 - Chapa tracionada com fenda.

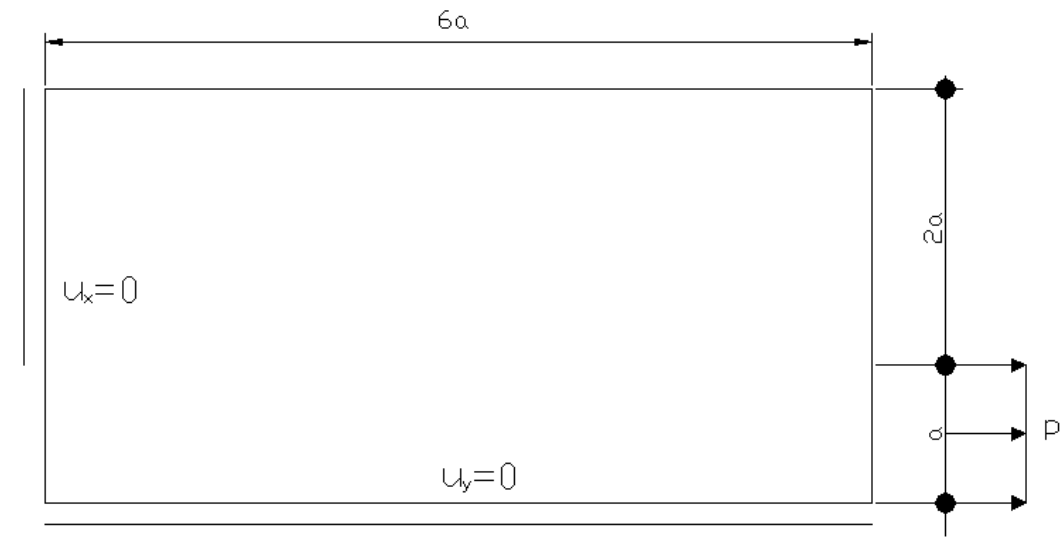

Figura 7.3 - Dupla simetria da chapa tracionada com fenda.

Como na FHMT são aproximados de forma independente três campos, o enriquecimento dessas aproximações pode ser explorado também com diferentes combinações. Neste estudo, seis possibilidades independentes são empregadas: o enriquecimento sobre o campo de tensões no domínio, sobre o campo de tensões e deslocamento no domínio, sobre o campo de tensões no domínio e deslocamento no contorno, sobre o campo de deslocamento no domínio e sobre o campo de deslocamento no contorno. O Teste do Mosaico, apresentado no capítulo anterior, é aplicado para 
todas as possibilidades de enriquecimento, como ponto inicial de análise da solvibilidade dos dois problemas.

Vale lembrar que, por simplificação, o enriquecimento dos campos de tensão e deslocamento no domínio é simultâneo e realizado nos mesmos nós, ou seja, ao enriquecer a base de aproximação do campo de tensões em um nó a base de aproximação do campo de deslocamento também é enriquecida.

Para análise dos problemas 1 e 2, utilizam-se malhas regulares e irregulares, com os elementos quadrilaterais de quatro nós definidos no trabalho. Os campos de tensão de cada problema são comparados com resultados de uma análise pelo MEF convencional, gerados no software ANSYS ${ }^{\circledR}$, a partir de uma malha bastante refinada. A energia de deformação é adotada como medida global de comparação entre as simulações. Por exemplo, no caso do problema 2, a energia de deformação obtida numa análise por meio da FHMT é confrontada com a energia de deformação exata, dada pela seguinte relação: $\boldsymbol{U}_{e x}^{2} \approx 0,0458403 \frac{(6 \boldsymbol{p a})^{2}}{E}$.

Além disso, em cada um dos problemas simulado com o ANSYS $^{\circledR}$ são identificados deslocamentos em nós de referência para a confrontação com os respectivos deslocamentos da FHMT com enriquecimento nodal.

Para a integração numérica de todas as matrizes envolvidas, nos ensaios numéricos sem enriquecimento, utilizam-se 2 pontos de Gauss em cada direção do elemento de domínio e 2 pontos de Gauss em cada elemento de contorno. Quando do enriquecimento polinomial sobre as bases de aproximação do domínio, usam-se $\boldsymbol{g}_{a p}+\mathbf{2}$ pontos de Gauss em cada direção do elemento de domínio para integração, onde $\boldsymbol{g}_{a p}$ é o grau máximo das aproximações enriquecidas. Para a base aproximada do contorno, também enriquecida com funções polinomiais, utilizam-se $\boldsymbol{g}_{a p \Gamma}+2$ pontos de Gauss para integração, onde $\boldsymbol{g}_{a p \Gamma}$ é o grau máximo das aproximações enriquecidas para o contorno.

No caso de enriquecimento das aproximações do domínio com as funções que se assemelham às soluções da fratura (eq.(5.37), eq.(5.38), eq.(5.39), eq.(5.40) e eq.(5.41)), como nenhum procedimento adaptativo específico de integração numérica é utilizado (a exemplo do procedimento aplicado no trabalho de Strouboulis e Babuška e Copps (2002)), opta-se por usar uma grande quantidade de pontos de Gauss para integração numérica. Nesse trabalho, para esse tipo de função enriquecedora, utilizam-se 50 pontos 
de Gauss em cada direção no caso do elemento de domínio e 25 pontos de Gauss no caso do elemento de contorno.

Quando o enriquecimento das bases aproximativas do campo de tensões for desenvolvido com algum campo de tensões auto-equlibradas, utilizam-se, para integração numérica das matrizes, os mesmos procedimentos quando do enriquecimento polinomial.

Adotando-se funções trigonométricas para o enriquecimento das bases aproximativas da FHMT, usam-se, no mínimo, 5 pontos de Gauss em cada direção do elemento de domínio e 3 pontos de Gauss para o elemento de contorno.

\subsection{Problema 1: Chapa Tracionada Simetricamente}

Para a geração com o ANSYS $^{\circledR}$ de resultados de referência para o problema 1 , considerou-se a discretização representada na figura 7.4.

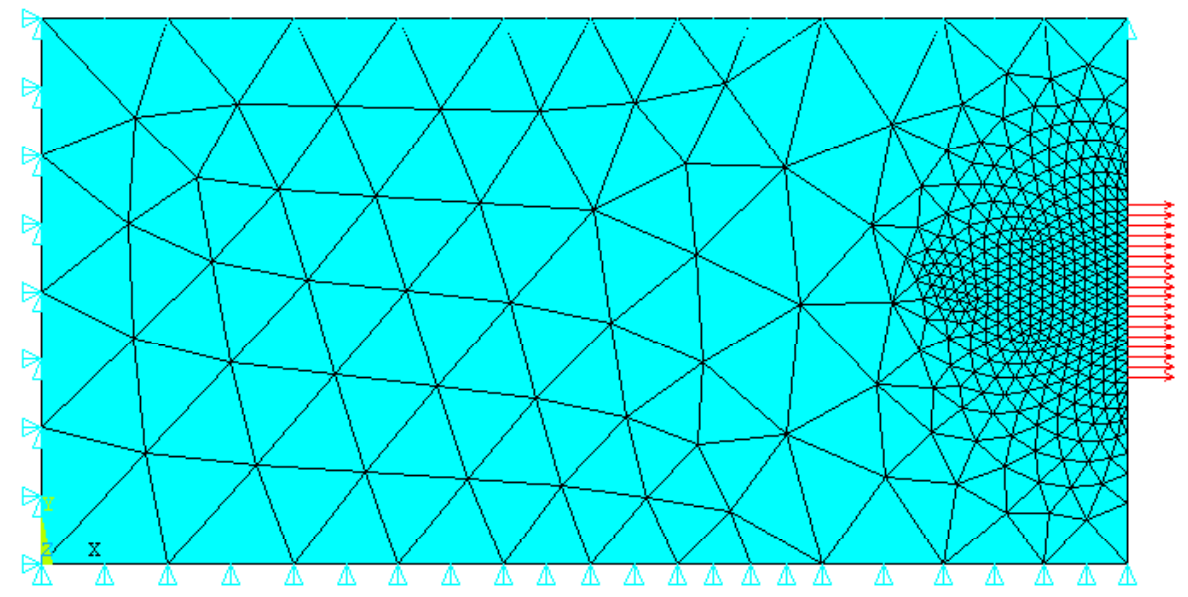

Figura 7.4 - Exemplo modelo do problema 1.

Nessa discretização um total de 798 elementos triangulares foram distribuídos com um maior refinamento na região da aplicação do carregamento. $O$ elemento triangular Plane 2 da biblioteca do ANSYS $^{\circledR}$ foi utilizado na análise, possuindo seis nós e dois graus de liberdade por nó $\left(\boldsymbol{u}_{\boldsymbol{x}}\right.$ e $\boldsymbol{u}_{\boldsymbol{y}}$ ). Como valor de referência para a energia de deformação, obteve-se: 46,47. Para os deslocamentos de referência adotaram-se os graus de liberdade do nó do canto superior direito da chapa, obtendo-se, para essa nova situação, como valores: $\boldsymbol{u}_{\boldsymbol{x}}=0.2818$ e $\boldsymbol{u}_{\boldsymbol{y}}=0.0000$.

A resposta para o campo de tensão, para esse exemplo modelo, está ilustrada na figura 7.5 . 


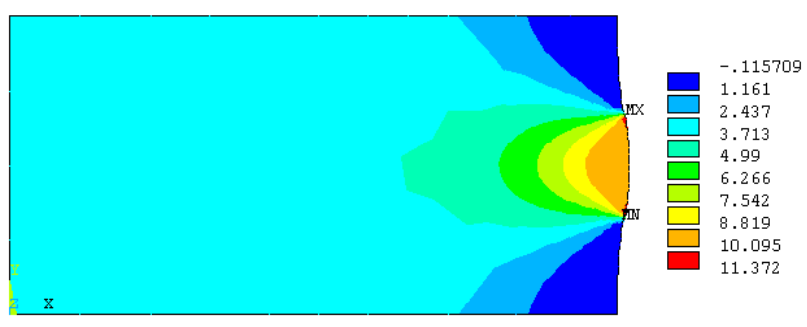

Sigma $-\mathrm{x}$

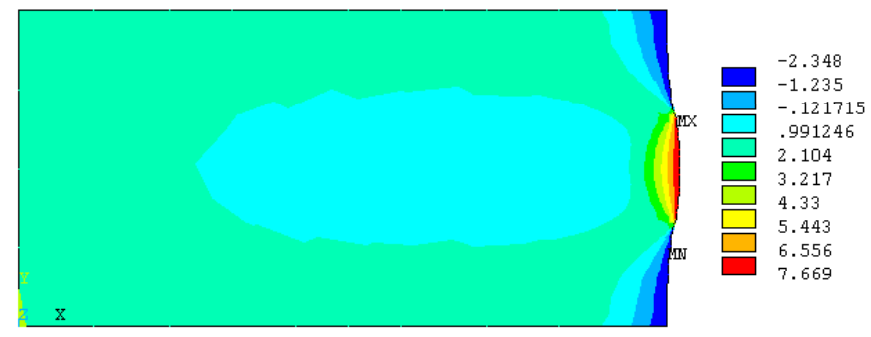

Sigma $-\mathrm{y}$

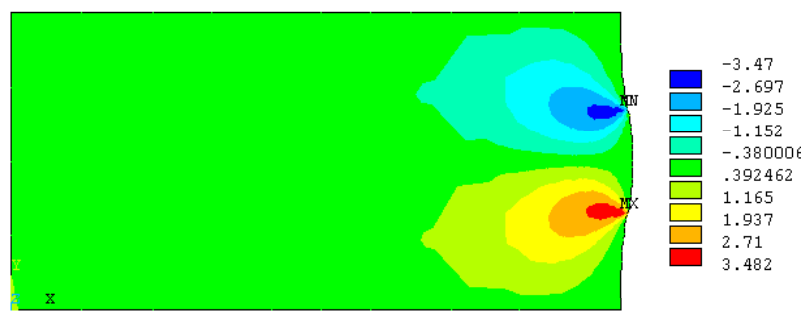

$\mathrm{Tau}-\mathrm{xy}$

Figura 7.5 - Exemplo modelo do problema 1 - representação do campo de tensões.

Para análise do problema 1 com a FHMT com enriquecimento nodal, duas possibilidades de malha são selecionadas: a primeira é uma malha regular com oito divisões na direção $\boldsymbol{x}$ e nove na direção $\boldsymbol{y}$ e a segunda é uma malha irregular com doze divisões na direção $\boldsymbol{x}$ e quinze na direção $\boldsymbol{y}$. A figura 7.6 apresenta as discretizações adotadas.

Neste problema, qualquer nó da malha de domínio pode ser enriquecido, mas os enriquecimentos dos nós da malha de contorno são realizados somente no lado vertical direito da chapa, onde não existem condições de contorno essenciais prescritas.

Quanto à aplicação do Teste do Mosaico, para as várias condições de enriquecimento, observa-se que para uma malha geral o teste deve ser aplicado tanto ao conjunto total de graus de liberdade, como a cada elemento ou Mosaicos (nuvens) isoladamente. Para um melhor entendimento no tocante à aplicação desse teste, consideram-se os graus de liberdade de um único elemento retangular no domínio: doze graus de liberdade de tensão $\left(s_{\Omega}=12\right)$ e oito graus de liberdade de deslocamento $\left(\boldsymbol{q}_{\Omega}=\boldsymbol{8}\right)$. Sejam também os graus de liberdade de um único elemento de contorno: quatro graus de liberdade de deslocamento $\left(\boldsymbol{q}_{\Omega}=4\right)$.

Admite-se que esses dois elementos estejam sobrepostos de modo que o elemento de contorno ocupe um dos lados do elemento de domínio. 


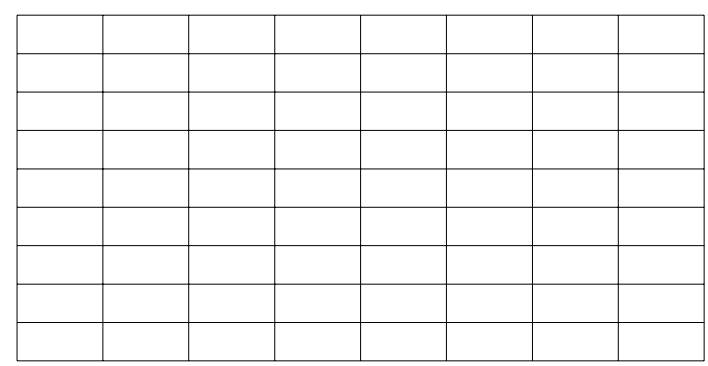

Malha regular $8 \times 9$

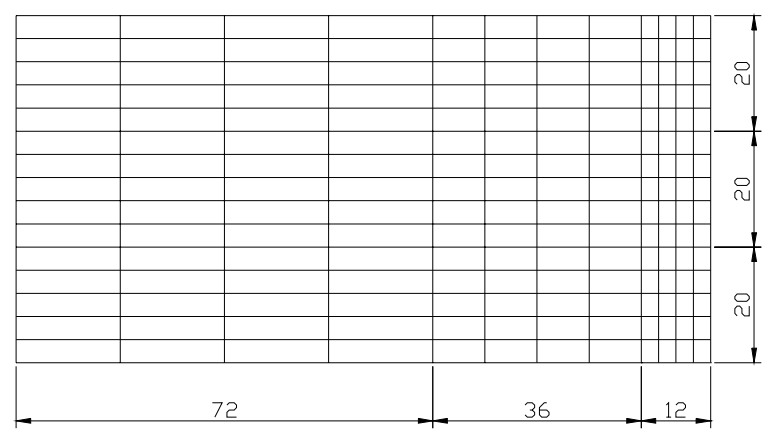

Malha irregular $12 \times 15$

Figura 8.6 - Discretizações adotadas para análise do problema 1.

Para o elemento de domínio, numa situação sem enriquecimento, a eq.(6.15) deve ser satisfeita ou seja: $\boldsymbol{s}_{\Omega} \geq \boldsymbol{q}_{\Omega} \Rightarrow(12 \geq 8)$. No elemento de contorno, sem enriquecimento, tem-se que a eq.(6.17) deve ser satisfeita ou seja: $\boldsymbol{s}_{\Omega} \geq \boldsymbol{q}_{\Gamma} \Rightarrow(6 \geq 4)$.

Nesta última condição, consideram-se seis graus de liberdade de tensão $\left(s_{\Omega}=6\right.$ ), pois somente os nós da malha de domínio que coincidem com os nós da malha de contorno contribuem para a montagem da matriz $\boldsymbol{A}_{\Gamma_{i}}$ o elemento $\boldsymbol{i}$ no contorno.

Agora, analisando-se o enriquecimento em suas várias possibilidades de combinação e considerando-se as condições expressas pelas eq.(6.15) e eq.(6.17), podese concluir inicialmente que:

- O enriquecimento exclusivo sobre o campo de tensões no domínio é livre, pois as eq.(6.15) e eq.(6.17) são sempre satisfeitas para qualquer parcela analisada;

- O enriquecimento simultâneo dos campos de tensão e deslocamento no domínio também é livre, pois a eq.(6.15) é satisfeita em todas as parcelas;

- Enriquecer simultaneamente os campos de tensão e deslocamento no domínio e o deslocamento no contorno é permitido, desde que os nós da malha de domínio que coincidem com os nós da malha de contorno enriquecidos, também sejam enriquecidos. Basta que dois nós de um elemento do contorno, sem condições de contorno essenciais prescritas, 
sejam enriquecidos sem essa preocupação e a condição dada pela eq.(6.17) será violada;

- Para o enriquecimento exclusivo dos campos de tensões no domínio e de deslocamentos no contorno valem as mesmas observações da conclusão anterior;

- Enriquecer somente o campo de deslocamentos no domínio, ou o campo de deslocamentos no domínio juntamente com o campo de deslocamentos no contorno ou ainda somente o campo de deslocamentos no contorno não é recomendável, pois para poucos nós enriquecidos no domínio e contorno considerando-se um elemento, as eq.(6.15) e eq.(6.17) já não serão mais verificadas.

É importante ressaltar que as conclusões anteriores são necessárias, mas não suficientes para a garantia de solvibilidade do sistema de equações lineares, dado pela eq. (3.32), e conseqüentemente da estabilidade da resposta pela FHMT. Por exemplo, as funções adotadas para o enriquecimento podem ter influência na estabilidade da resposta.

Apresenta-se em seguida, para cada uma das duas malhas utilizadas na análise do problema 1, um resumo contendo os resultados de várias investigações, no tocante às possibilidades de enriquecimento com a utilização do Teste do Mosaico.

Para leitura da tabela de resultados, considere-se a seguinte legenda:

- $\Omega$ : domínio;

$-\Gamma$ : contorno;

- $\sigma$ : aproximação do campo de tensão;

- $u$ : aproximação campo de deslocamento;

- Energia de deformação do exemplo modelo: $\boldsymbol{U}_{E M}=46,47$;

- Deslocamentos de referência do exemplo modelo: $\boldsymbol{u}_{x E M}=0.2818$ e $\boldsymbol{u}_{y E M}=0.0000$.

- Resultados para a malha regular 8x9 - enriquecimento polinomial com diferentes funções no domínio e com $\left(y-y_{n \dot{b}}\right)^{2}$ no contorno. 
Como função enriquecedora dos campos de domínio, adota-se inicialmente, a função do tipo $\left(y-y_{n o ́}\right)^{2}$.

Oito nós específicos da malha de cobertura de domínio são escolhidos para realização dos vários tipos de enriquecimento.Tais nós são: 35, 36, 44, 45, 53, 54, 62 e 63. Na malha de cobertura de contorno são quatro os nós enriquecidos: 36, 45, 54 e 63. Esses nós ficam próximos da região onde está aplicado o carregamento da chapa; como mostra a figura 7.7 .

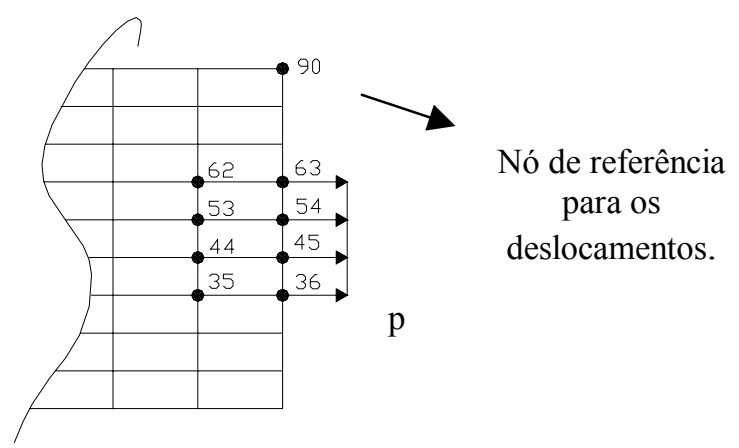

Figura 7.7 - Nós da malha regular 8x9 escolhidos no enriquecimento seletivo.

Vale lembrar que a numeração dos nós e elementos adotados no programa fonte é a seguinte: para a malha de cobertura de domínio as numerações dos nós e elementos são feitas da esquerda para a direita e de baixo para cima; já os nós de contorno recebem a mesma numeração que foi dada aos nós de domínio coincidentes. Para numeração dos elementos de contorno, adota-se o sentido anti-horário, sendo o primeiro elemento aquele em coincidência com o primeiro lado horizontal do elemento de domínio localizado no lado inferior esquerdo da chapa.

Os resultados da tabela 7.1 mostram que, em todas as situações onde o Teste do Mosaico falha a energia de deformação se afasta do valor de referência, ou, então, o sistema apresentou problemas de convergência para a tolerância imposta. Nota-se que o enriquecimento exclusivo sobre o campo de tensões e deslocamentos no domínio proporciona um valor de energia de deformação mais próximo ao valor de referência, quando comparados com as energias obtidas com enriquecimento exclusivo sobre o campo de tensão.

A tabela 7.1 também apresenta, para fins de comparação, resultados obtidos com todos os nós de domínio enriquecidos. 
Tabela 7.1 - Resultados gerais obtidos para o problema 1 com malha regular $8 \times 9$ e função enriquecedora do tipo $\left(y-y_{n \dot{ }}\right)^{2}$.

\begin{tabular}{ccc}
\hline Condições de Enriquecimento & Energia de Deformação & Teste do Mosaico \\
\cline { 2 - 3 } Sem enriquecimento & 46,52 & Ok! \\
\hline Todos os nós enriquecidos $-\sigma$ e $u-\Omega$ & 45,98 & Ok! \\
\hline Todos os nós enriquecidos $-\sigma-\Omega$ & 45,34 & Ok! \\
\hline Todos os nós enriquecidos $-u-\Omega$ & 104,64 & Não Ok! \\
\hline Oito nós enriquecidos $-\sigma$ e $u-\Omega$ & 46,06 & Ok! \\
\hline Oito nós enriquecidos - $\sigma-\Omega$ & 45,79 & Não Ok! \\
\hline Oito nós enriquecidos $-u-\Omega$ & 63,72 & Ok! \\
\hline $\begin{array}{c}\text { Oito nós enriquecidos }-\sigma \text { e } u-\Omega \text { e } \\
\text { quatro nós }-u \text { no } \Gamma\end{array}$ & 46,75 & Não Ok! \\
\hline $\begin{array}{c}\text { Oito nós enriquecidos }-\sigma-\Omega \text { e } \\
\text { quatro nós }-u \text { no } \Gamma\end{array}$ & 46,52 & Não Ok! \\
\hline $\begin{array}{c}\text { Oito nós enriquecidos }-u-\Omega \text { e } \\
\text { quatro nós }-u-\Gamma\end{array}$ & Não convergiu \\
\hline Quatro nós $-u-\Gamma$ & Não convergiu &
\end{tabular}

O enriquecimento do campo de deslocamento no contorno associado às outras possibilidades de enriquecimento, quando o Teste do Mosaico é satisfeito, gera valores de energia de deformação bem próximos aos valores de referência.

Na tabela 7.2, mostram-se os resultados do Teste do Mosaico para as mesmas condições de enriquecimento pontuadas na tabela 7.1, mas com uma diferente função enriquecedora para as aproximações dos campos de tensões e deslocamentos no domínio.

Nessas novas análises é adotada a seguinte função enriquecedora para as aproximações no domínio do problema 1: $\left(y-y_{n o ́}\right)$.

Tabela 7.2 - Resultados gerais obtidos para o problema 1 com malha regular $8 \times 9$ e função enriquecedora do tipo $\left(y-y_{n o ́}\right)$.

\begin{tabular}{ccc}
\hline Condições de Enriquecimento & Energia de Deformação & Teste do \\
& $\boldsymbol{U}_{E M}=46,47$ & Mosaico \\
\hline Sem enriquecimento & 46,52 & Ok! \\
\hline Todos os nós enriquecidos $-\sigma$ e $u-\Omega$ & 46,19 & Ok! \\
\hline Todos os nós enriquecidos $-\sigma-\Omega$ & 45,76 & Ok! \\
\hline Todos os nós enriquecidos $-u-\Omega$ & 104,64 & Não Ok! \\
\hline Oito nós enriquecidos $-\sigma$ e $u-\Omega$ & 46,20 & Ok! \\
\hline Oito nós enriquecidos $-\sigma-\Omega$ & 45,91 & Ok! \\
\hline Oito nós enriquecidos $-u-\Omega$ & 63,72 & Não Ok! \\
\hline Oito nós enriquecidos $-\sigma$ e $u-\Omega$ e quatro nós $-u-\Gamma$ & 46,93 & Ok! \\
\hline Oito nós enriquecidos $-\sigma-\Omega$ e quatro nós $-u-\Gamma$ & 46,53 & Ok! \\
\hline Oito nós enriquecidos $-u-\Omega$ e quatro nós $-u-\Gamma$ & Não convergiu & Não Ok! \\
\hline Quatro nós $-u-\Gamma$ & Não convergiu & Não Ok! \\
\hline
\end{tabular}


As respostas indicadas na tabela 7.2 têm praticamente o mesmo comportamento daquelas apresentadas na tabela 7.1.

Como pode ser visto na figura 7.7, o nó 90 (nó do canto superior direito da chapa) é o nó de referência para efeito de comparação dos campos de deslocamentos. Na tabela 7.3 são apresentados os resultados dos deslocamentos do nó de referência para a malha regular 8x9 com a consideração dos diferentes tipos de funções enriquecedoras.

Tabela 7.3 - Deslocamentos do nó de referência da malha de cobertura de domínio e contorno para a malha regular $8 \times 9$ e função enriquecedora do tipo $\left(y-y_{n \dot{\prime}}\right)^{2}$.

\begin{tabular}{|c|c|c|}
\hline \multirow{3}{*}{ Condição de Enriquecimento } & \multicolumn{2}{|c|}{ Deslocamentos do nó de referência } \\
\hline & \multicolumn{2}{|c|}{$u_{x E M}=0,2818$ e $u_{y E M}=0,0000$} \\
\hline & Deslocamento do nó $90(\Gamma)$ & Deslocamento do nó $90(\Omega)$ \\
\hline Sem enriquecimento & $u_{x}=0,2386$ e $u_{y}=0,0000$ & $u_{x}=0,2316$ e $u_{y}=-0,1147$ \\
\hline Todos os nós enriquecidos - $\sigma$ e $u-\Omega$ & $u_{x}=0,2949$ e $u_{y}=0,0000$ & $u_{x}=0,2952$ e $u_{y}=-0,0077$ \\
\hline Todos os nós enriquecidos - $\sigma-\Omega$ & $u_{x}=0,2859$ e $u_{y}=0,0000$ & $u_{x}=0,2826 \mathrm{e} u_{y}=0,0003$ \\
\hline Todos os nós enriquecidos - $u-\Omega$ & $u_{x}=-0,0595$ e $u_{y}=0,0000$ & $u_{x}=-1,3540$ e $u_{y}=-4,1331$ \\
\hline Oito nós enriquecidos - $\sigma$ e $u-\Omega$ & $u_{x}=0,3026 \mathrm{e} u_{y}=0,0000$ & $u_{x}=0,2831$ e $u_{y}=0,0558$ \\
\hline Oito nós enriquecidos $-\sigma-\Omega$ & $u_{x}=0,3087$ e $u_{y}=0,0000$ & $u_{x}=0,3172 \mathrm{e} u_{y}=0,0165$ \\
\hline Oito nós enriquecidos - $u-\Omega$ & $u_{x}=0,0875$ e $u_{y}=0,0000$ & $u_{x}=-1,2541$ e $u_{y}=-5,9203$ \\
\hline $\begin{array}{c}\text { Oito nós enriquecidos - } \sigma \text { e } u-\Omega \text { e } \\
\text { quatro nós }-u-\Gamma\end{array}$ & $u_{x}=0,2332$ e $u_{y}=0,0000$ & $u_{x}=0,2231$ e $u_{y}=-0,1117$ \\
\hline $\begin{array}{c}\text { Oito nós enriquecidos - } \sigma-\Omega \text { e quatro } \\
\text { nós }-u-\Gamma\end{array}$ & $u_{x}=0,2437$ e $u_{y}=0,0000$ & $u_{x}=0,1115$ e $u_{y}=0,1637$ \\
\hline
\end{tabular}

Os deslocamentos de referência do nó 90 obtidos com os enriquecimentos do tipo $\left(y-y_{n \dot{b}}\right)^{2}$ e $\left(y-y_{n \dot{o}}\right)$ são muito semelhantes, e, em termos gerais, próximos aos deslocamentos de referência dos exemplos modelos.

Com o enriquecimento somente sobre a aproximação do campo de deslocamento no domínio, os deslocamentos de referência do nó 90 da malha de contorno e domínio não apresentam compatibilidade e nem proximidade com os valores dos deslocamentos do exemplo de referência. Nas situações onde o Teste do Mosaico é satisfeito, dos resultados da tabela 7.3, tem-se que o enriquecimento compatibiliza os valores dos deslocamentos de referência do nó 90 da malha de contorno e domínio. Esses valores são incompatíveis quando não há enriquecimento.

Observando-se os resultados da tabela 7.3, fica evidente que o enriquecimento exclusivo sobre o campo de deslocamentos no domínio não fornece bons resultados (Teste do Mosaico não satisfeito). 
A seguir serão apresentadas visualizações do campo de tensões para algumas das situações de enriquecimento estudadas. Na figura 7.8, tem-se a representação do campo de tensões para a situação sem enriquecimento.

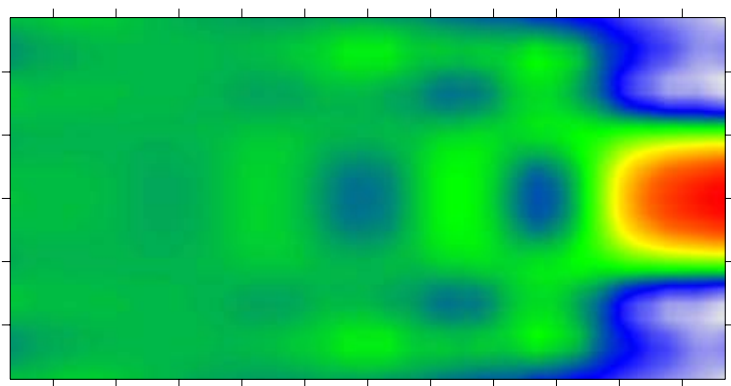

Sigma-X

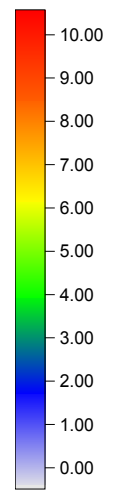

Sigma-y

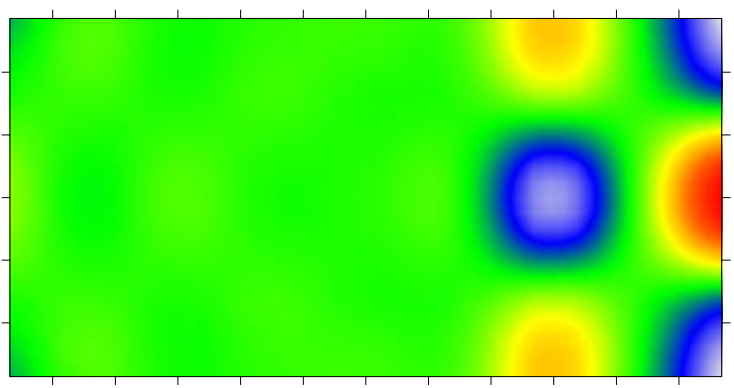

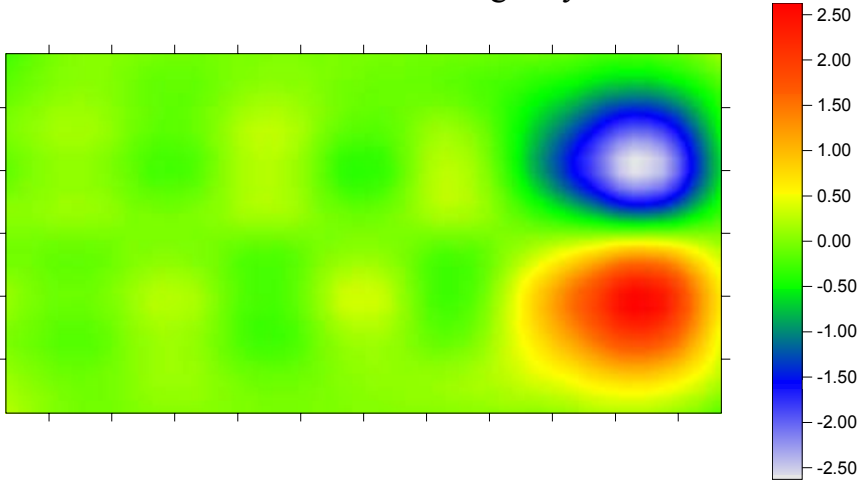

Tau-xy

Figura 7.8 - Representação do campo de tensões sem enriquecimento.

Com o enriquecimento sobre os campos de tensão e deslocamento no domínio, (ver figura 7.9) há um pequeno acréscimo na estimativa dos valores máximos dos campos de tensões.

Enriquecendo-se o campo de deslocamento no contorno, também há um aumento na estimativa dos valores máximos dos campos de tensão, além de uma melhor definição do bulbo de tensões próximo a região da aplicação do carregamento. Uma visualização dos campos de tensão para essa situação será apresentada com a malha irregular $12 \times 15$. 

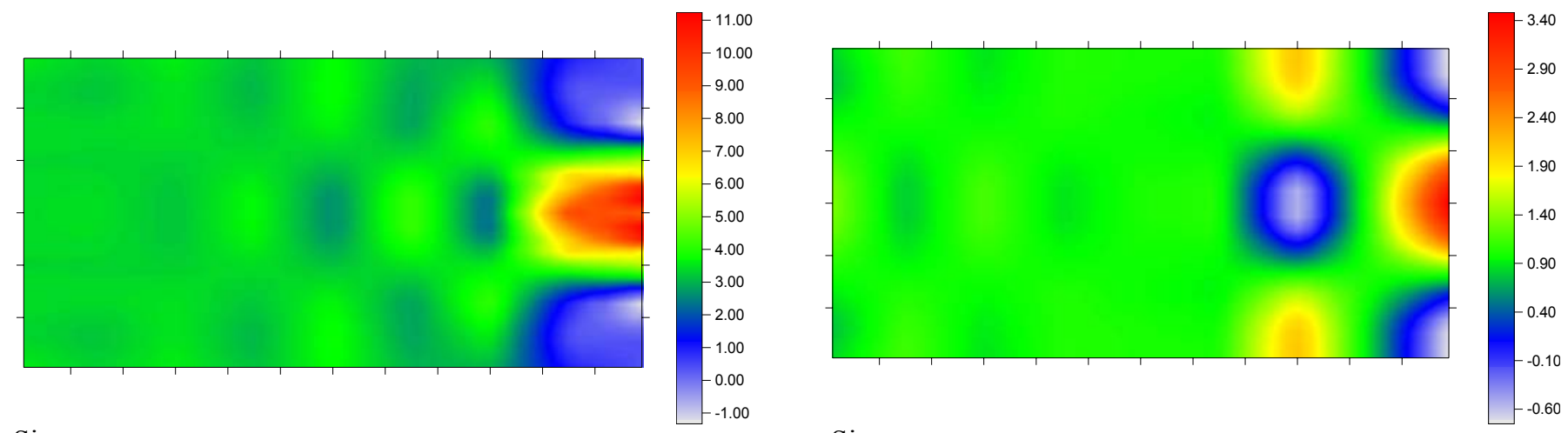

Sigma-X

Sigma-y

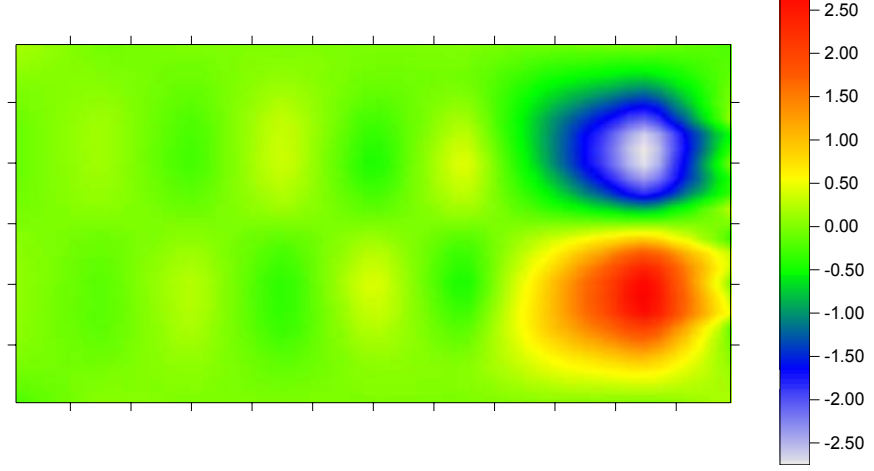

Tau $-\mathrm{xy}$

Figura 7.9 - Representação do campo de tensões com enriquecimento do tipo $\left(y-y_{n o ́}\right)^{2}$ sobre $\sigma$ e $u$ no $\Omega$ - oito nós (35,36,44,45,53,54,62 e 63).

- Resultados para a malha regular 8x9 - enriquecimento com funções de Airy com o grau de enriquecimento $\boldsymbol{d}_{e}=\mathbf{1}$ e $\boldsymbol{d}_{e}=\mathbf{2}$.

Nas tabelas 7.4 e 7.5 mostram-se os resultados gerais dos ensaios numéricos realizados com a possibilidade de enriquecimento das bases aproximativas dos campos de tensão no domínio com os polinômios correspondentes a funções de Airy.

Tabela 7.4 - Resultados gerais obtidos para o problema 1 com malha regular $8 \times 9 \mathrm{e}$ função de Airy enriquecedora do tipo polinomial $\left(\boldsymbol{d}_{e}=1\right)$.

\begin{tabular}{ccc}
\hline Condições de Enriquecimento & Energia de Deformação & Teste do Mosaico \\
\cline { 2 - 3 } & $\boldsymbol{U}_{\boldsymbol{E}}=46,47$ & $\mathrm{Ok} !$ \\
\hline Sem enriquecimento & 46,52 & $\mathrm{Ok} !$ \\
\hline Todos os nós enriquecidos $-\sigma-\Omega$ & 44,07 & $\mathrm{Ok} !$ \\
\hline $\begin{array}{c}\text { Oito nós enriquecidos }-\sigma-\Omega \\
(35,36,44,45,53,54,62 \text { e } 63)\end{array}$ & 44,67 & $\mathrm{Ok} !$ \\
\hline $\begin{array}{c}\text { Oito nós enriquecidos }-\sigma-\Omega \\
(34,35,343,44,52,53,61 \text { e } 62)\end{array}$ & 44,91 & \\
\hline
\end{tabular}

$\mathrm{Na}$ tabela 7.4 para as condições de enriquecimento apresentadas, utiliza-se o grau de enriquecimento $\boldsymbol{d}_{e}=1$ e conseqüentemente 4 funções $\left(\boldsymbol{n}_{e}=4\right)$ enriquecem a 
base aproximativa inicial dos campos de tensões. Já os resultados do tabela 7.5 são obtidos com o grau de enriquecimento $\boldsymbol{d}_{\boldsymbol{e}}=\mathbf{2}$ e assim, 9 funções $\left(\boldsymbol{n}_{\boldsymbol{e}}=\boldsymbol{9}\right)$ enriquecem as aproximações do campo de tensões. Observa-se que além dos nós propostos para enriquecimento seletivo (ver figura 7.7) outras situações de enriquecimento são apresentadas para efeito de comparação.

Tabela 7.5 - Resultados gerais obtidos para o problema 1 com malha regular 8x9 e função de Airy enriquecedora do tipo polinomial $\left(\boldsymbol{d}_{e}=2\right)$.

\begin{tabular}{ccc}
\hline Condições de Enriquecimento & Energia de Deformação & \multirow{2}{*}{ Teste do Mosaico } \\
\cline { 2 - 3 } & $\boldsymbol{U}_{E M}=46,47$ & Ok! \\
\hline Sem enriquecimento & 46,52 & Ok! \\
\hline Todos os nós enriquecidos $-\sigma-\Omega$ & 42,27 & Ok! \\
\hline $\begin{array}{c}\text { Oito nós enriquecidos }-\sigma-\Omega \\
(35,36,44,45,53,54,62 \text { e } 63)\end{array}$ & 43,85 & Ok! \\
\hline $\begin{array}{c}\text { Oito nós enriquecidos - } \sigma-\Omega \\
(34,35,343,44,52,53,61 \text { e } 62)\end{array}$ & 44,31 & \\
\hline
\end{tabular}

Para esse tipo de enriquecimento as estimativas das energias de deformação obtidas são mais baixas quando comparadas com as energias resultantes dos ensaios numéricos com enriquecimentos polinomiais. Observa-se que para $\boldsymbol{d}_{\boldsymbol{e}}=\mathbf{2}$ as estimativas da energia de deformação são menores que as obtidas para $\boldsymbol{d}_{\boldsymbol{e}}=\boldsymbol{1}$.

Com relação aos deslocamentos de referência do nó 90, existe uma grande semelhança entre os resultados das duas situações de enriquecimento analisadas $\left(\boldsymbol{d}_{\boldsymbol{e}}=\mathbf{1}\right.$ e $\boldsymbol{d}_{\boldsymbol{e}}=2$ ) e, de uma forma geral, estão também próximos aos deslocamentos do nó referência do exemplo modelo.

Nas figuras 7.10, 7.11 e 7.12, destacam-se as representações do campo de tensões com $\boldsymbol{d}_{e}=\mathbf{1}$ e $\boldsymbol{d}_{e}=\mathbf{2}$. 

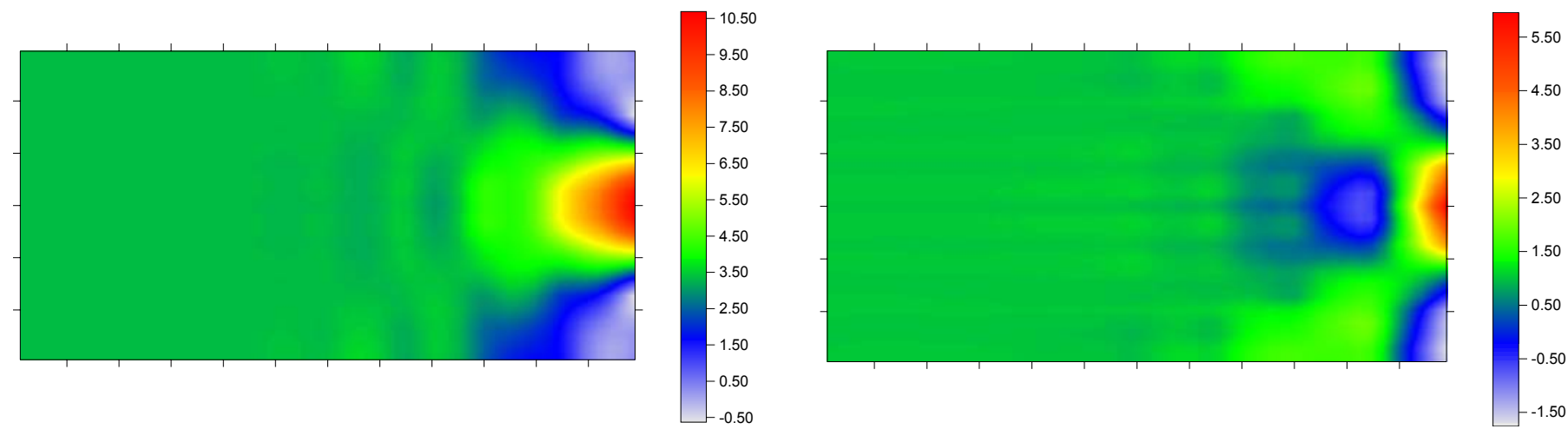

Sigma-x

Sigma-y

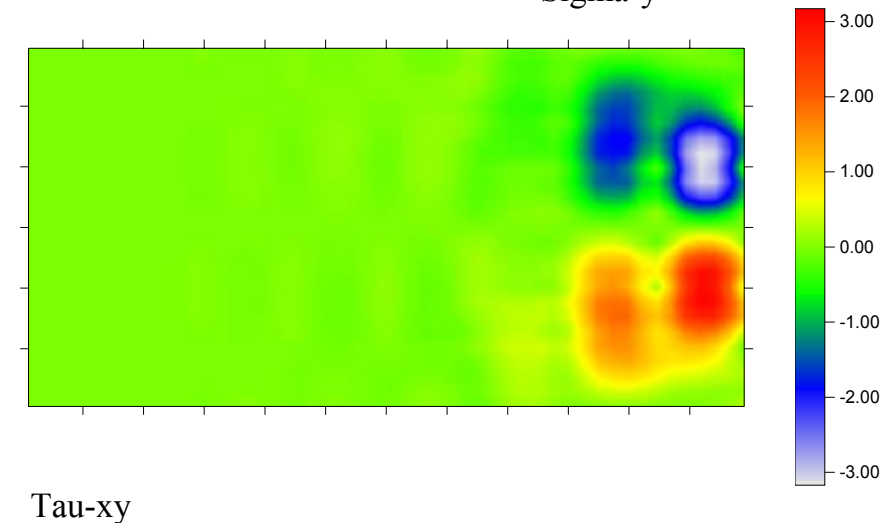

Tau-xy

Figura 7.10 - Representação do campo de tensões com enriquecimento do tipo $\boldsymbol{d}_{\boldsymbol{e}}=\mathbf{1}$ sobre $\sigma$ em todos os nós.
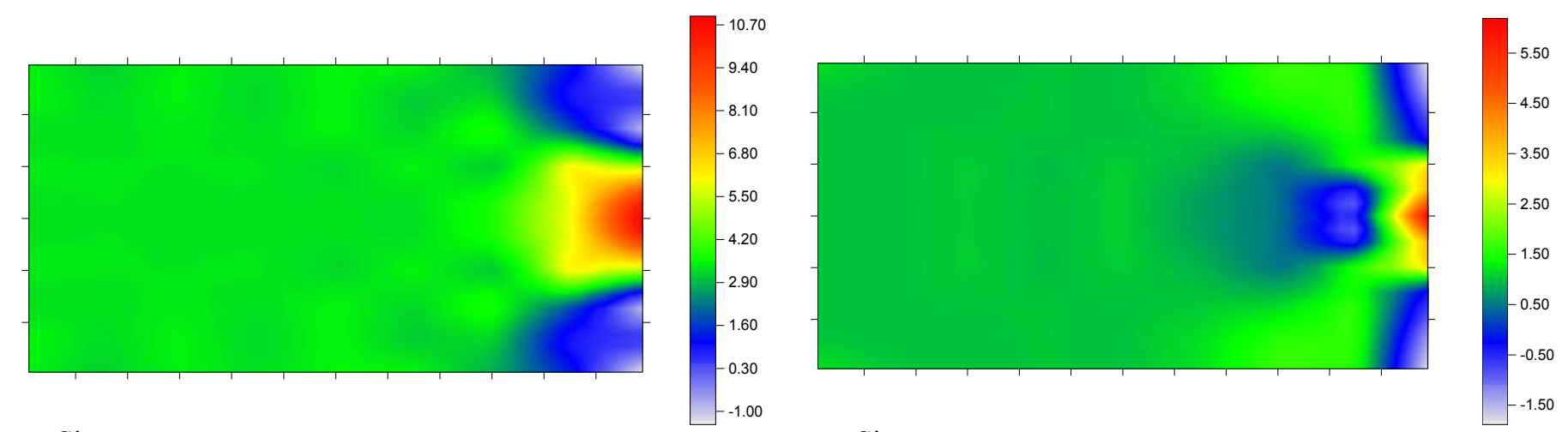

Sigma-x

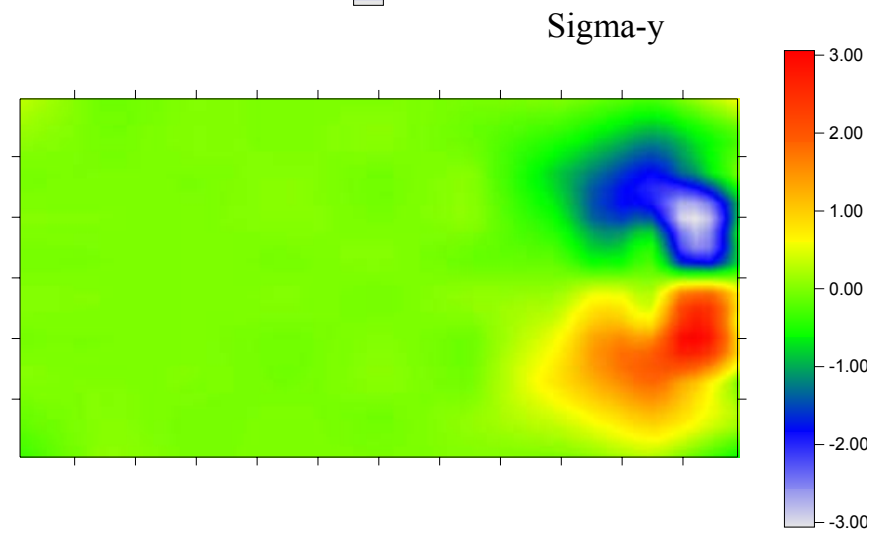

Tau-xy

Figura 7.11- Representação do campo de tensões com enriquecimento do tipo $\boldsymbol{d}_{\boldsymbol{e}}=\mathbf{1}$ sobre $\sigma$ - oito nós $(35,36,44,45,53,54,62$ e 63$)$. 


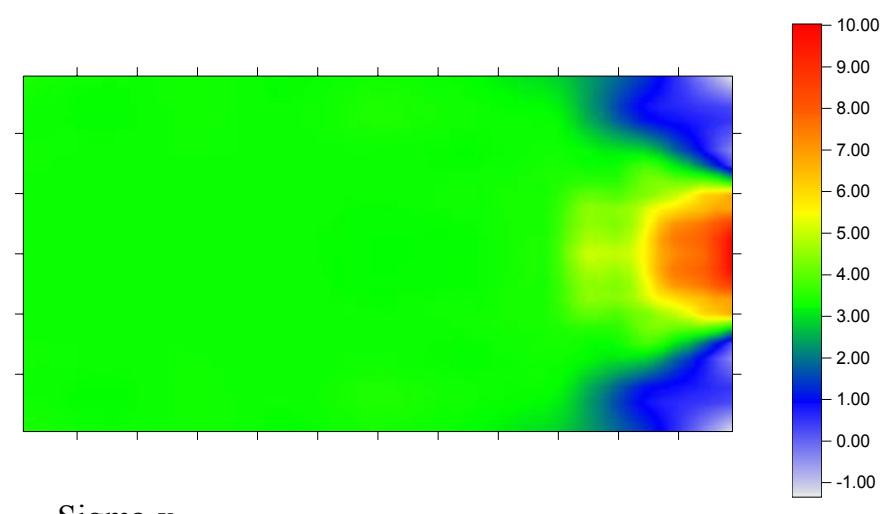

Sigma- $\mathrm{x}$

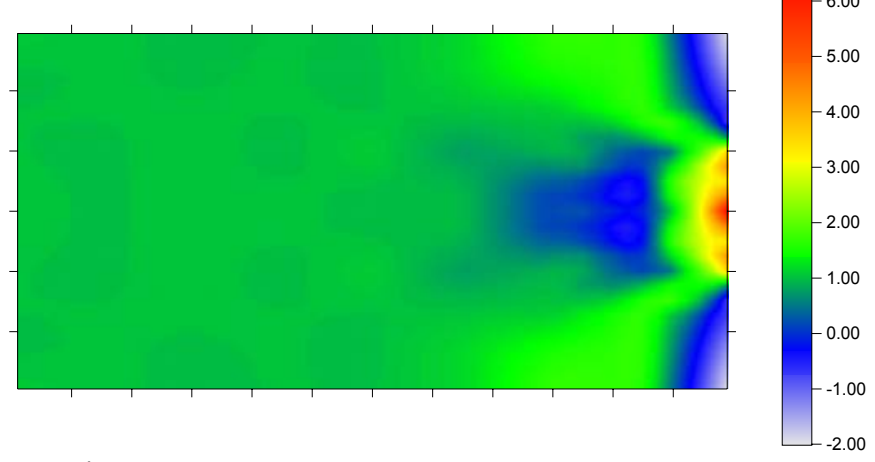

Sigma-y

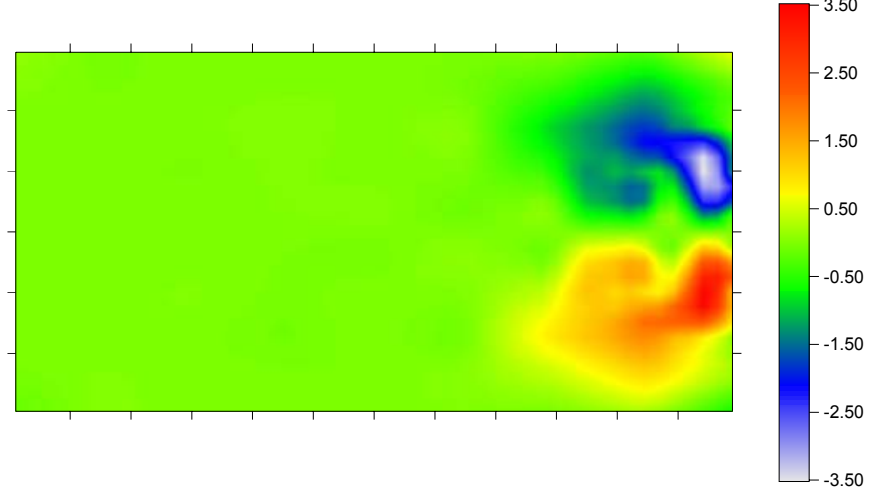

Tau-xy

Figura 7.12- Representação do campo de tensões com enriquecimento do tipo $\boldsymbol{d}_{\boldsymbol{e}}=\mathbf{2}$ sobre $\sigma$ - oito nós $(35,36,44,45,53,54,62$ e 63$)$.

Para todas as representações dos campos de tensões com enriquecimento pontuadas, verificou-se um aumento na estimativa dos valores máximos das tensões. As representações desses campos de tensões também tiveram uma boa aproximação quando comparados com o campo de tensões ilustrados na figura 7.5.

- Resultados para a malha irregular $12 \times 15$ - enriquecimento polinomial com a função: $\left(y-y_{n \dot{o}}\right)^{2}+\left(x-x_{n \dot{o}}\right)^{2}+\left(y-y_{n \dot{o}}\right)\left(x-x_{n \dot{o}}\right)$ no domínio e $\left(y-y_{n \dot{b}}\right)^{2}$ no contorno.

Os nós enriquecidos da malha irregular 12x15 também estão próximos à região de aplicação do carregamento. Enriquecem-se dezesseis nós na malha de domínio (64, $65,77,78,90,91,103,104,116,117,129,130,142,143,155$ e 156) e seis nós da malha de contorno $(78,91,104,117,130$ e 143). O nó de referência para os campos de deslocamento é o nó 208 (canto superior direito). O conjunto de nós enriquecidos está ilustrado na figura 7.13. 


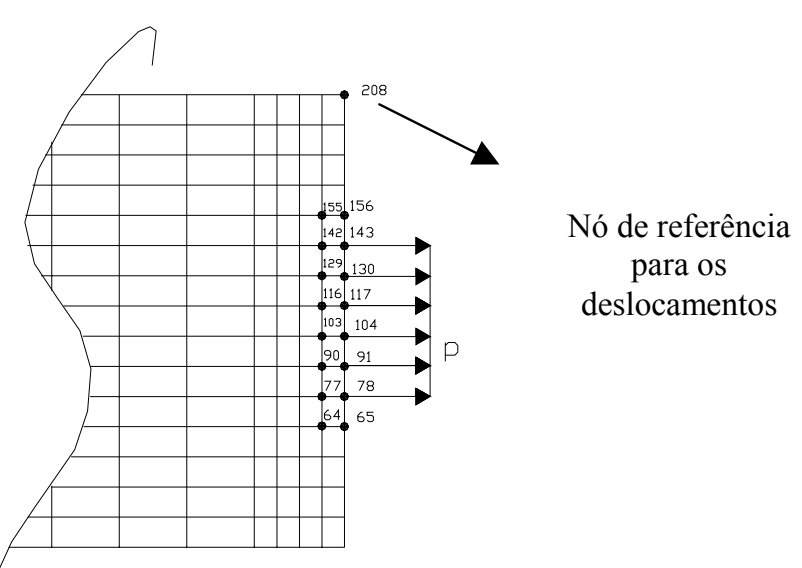

Figura 7.13 - Nós enriquecidos da malha irregular 12x15.

Tabela 7.6 - Resultados gerais obtidos para o problema 1 com malha irregular $12 \times 15$.

\begin{tabular}{|c|c|c|}
\hline \multirow{2}{*}{ Condições de Enriquecimento } & Energia de Deformação & \multirow[b]{2}{*}{ Teste do Mosaico } \\
\hline & $U_{E M}=46,47$ & \\
\hline Sem enriquecimento & 46,25 & Ok! \\
\hline Dezesseis nós enriquecidos $-\sigma$ e $u-\Omega$ & 46,15 & Ok! \\
\hline Dezesseis nós enriquecidos - $\sigma-\Omega$ & 46,04 & Ok! \\
\hline Dezesseis nós enriquecidos $-u-\Omega$ & 50,59 & Não Ok! \\
\hline $\begin{array}{c}\text { Dezesseis nós enriquecidos }-\sigma \text { e } u-\Omega \text { e } \\
\text { seis nós }-u \text { no } \Gamma\end{array}$ & 46,24 & Ok! \\
\hline $\begin{array}{l}\text { Dezesseis nós enriquecidos }-\sigma-\Omega \mathrm{e} \\
\text { quatro nós }-u \text { no } \Gamma\end{array}$ & 46,13 & Ok! \\
\hline $\begin{array}{l}\text { Oito nós enriquecidos }-u-\Omega \mathrm{e} \\
\text { quatro nós }-u-\Gamma\end{array}$ & Não convergiu & Não Ok! \\
\hline Quatro nós $-u-\Gamma$ & Não convergiu & Não Ok! \\
\hline
\end{tabular}

Com a malha irregular $12 \times 15$, os valores das energias de deformação, para as possibilidades de enriquecimento apresentadas na tabela 7.6, também estão próximos do valor da energia de deformação de referência. $O$ enriquecimento do tipo $\left(y-y_{n \dot{o}}\right)^{2}+\left(x-x_{n \dot{b}}\right)^{2}+\left(y-y_{n \dot{o}}\right)\left(x-x_{n \dot{o}}\right)$ simultâneo aos campos de domínio e contorno é mais eficiente.

Na tabela 7.7 estão agrupados os deslocamentos do nó de referência (208) para a malha irregular $12 \times 15$.

O enriquecimento compatibiliza os valores dos deslocamentos dos nós que pertencem simultaneamente às malhas de domínio e contorno, sempre que o Teste do Mosaico é verificado. Além disso, os deslocamentos de referência da malha irregular 12x15 também estão próximos aos deslocamentos de referência do exemplo modelo. 
Para o enriquecimento exclusivo sobre o campo de deslocamentos no domínio, como já era esperado, os deslocamentos do nó de referência observado como nó da malha de domínio ou de contorno não resultam compatíveis.

Tabela 7.7 - Deslocamentos do nó de referência da malha de cobertura de domínio e contorno para a malha irregular $12 \times 15$.

\begin{tabular}{|c|c|c|}
\hline \multirow{3}{*}{ Condições de Enriquecimento } & \multicolumn{2}{|c|}{ Deslocamentos do nó de referência } \\
\hline & \multicolumn{2}{|c|}{$\boldsymbol{u}_{x E M}=0,2818$ e $\boldsymbol{u}_{y E M}=0,0000$} \\
\hline & Deslocamento do nó $208(\Gamma)$ & Deslocamento do nó $208(\Omega)$ \\
\hline Sem enriquecimento & $\boldsymbol{u}_{\boldsymbol{x}}=\mathbf{0 , 2 8 6 8}$ e $u_{y}=0,0000$ & $u_{x}=0,2885$ e $u_{y}=-0,0119$ \\
\hline $\begin{array}{c}\text { Dezesseis nós enriquecidos }-\sigma \text { e } u- \\
\Omega\end{array}$ & $\boldsymbol{u}_{x}=0,3035$ e $u_{y}=0,0000$ & $u_{x}=0,2805$ e $u_{y}=0,0008$ \\
\hline Dezesseis nós enriquecidos - $\sigma-\Omega$ & $\boldsymbol{u}_{x}=0,3022$ e $u_{y}=0,0000$ & $u_{x}=0,3032$ e $u_{y}=-0,0007$ \\
\hline Dezesseis nós enriquecidos - $u-\Omega$ & $\boldsymbol{u}_{\boldsymbol{x}}=0,2277$ e $u_{y}=0,0000$ & $u_{x}=0.1674 \mathrm{e} u_{y}=-0,4989$ \\
\hline $\begin{array}{c}\text { Dezesseis nós enriquecidos - } \sigma \text { e } u-\Omega \\
\text { e seis nós }-u-\Gamma\end{array}$ & $u_{x}=0,3050$ e $u_{y}=0,0000$ & $u_{x}=0,3176$ e $u_{y}=-0,0012$ \\
\hline $\begin{array}{l}\text { Dezesseis nós enriquecidos - } \sigma-\Omega \mathrm{e} \\
\text { seis nós }-u-\Gamma\end{array}$ & $\boldsymbol{u}_{x}=0,3020$ e $u_{y}=0,0000$ & $u_{x}=0,3048 \mathrm{e} u_{y}=-0,0018$ \\
\hline
\end{tabular}

As visualizações dos campos de tensões, para a malha irregular 12x15 sem enriquecimento, estão representadas na figura 7.14. Nessa figura, observa-se que o sigma-x e o sigma-y estão com as zonas de concentrações de tensão, na região adjacente à aplicação do carregamento, bem mais definidas quando comparadas com as representações dos campos de tensões da malha regular apresentada anteriormente. 

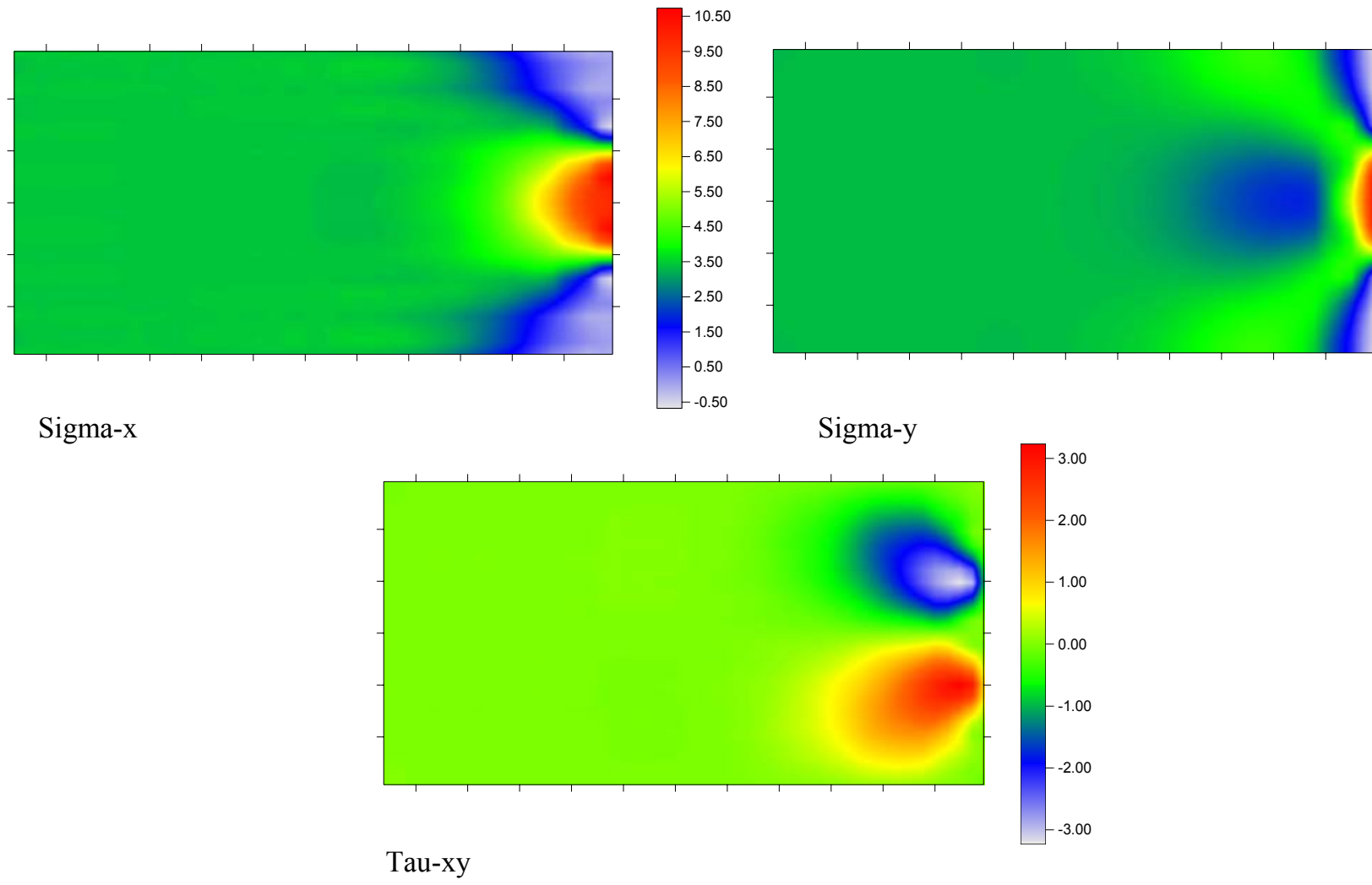

Figura 7.14 - Representação do campo de tensões sem enriquecimento.
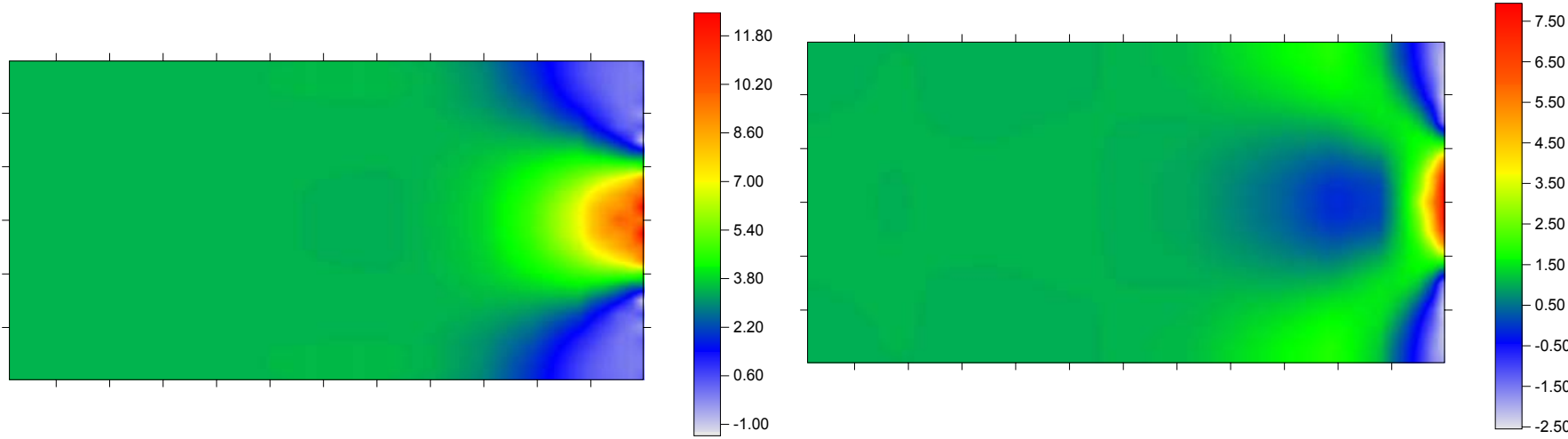

Sigma-X

Sigma-y
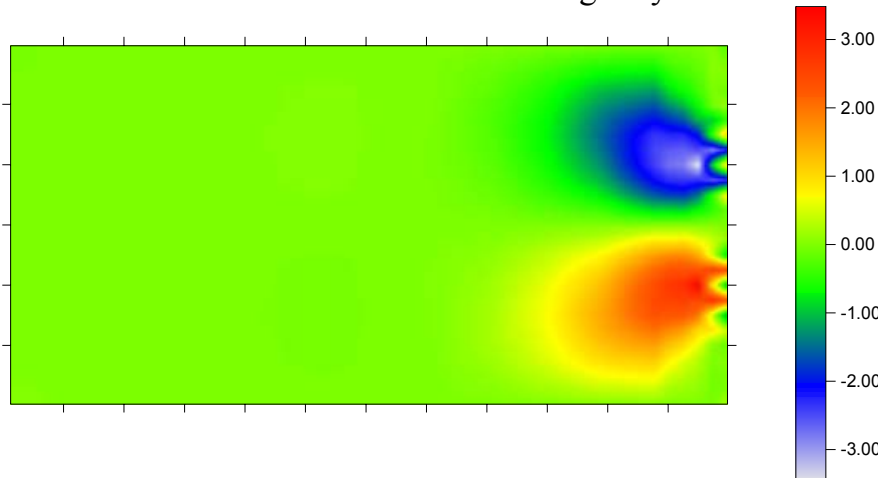

Tau-xy

Figura 7.15-Representação do campo de tensões com enriquecimento do tipo $\left(y-y_{n \dot{\delta}}\right)^{2}+\left(x-x_{n \dot{\delta}}\right)^{2}+\left(y-y_{n \dot{\delta}}\right)\left(x-x_{n \dot{\delta}}\right)$ sobre $\sigma$ no $\Omega$ - dezesseis nós $(64,65,77,78,90,91,103,104,116,117,129,130,142,143,155$ e 156$)$. 
$\mathrm{Na}$ figura 7.15, pode-se notar um aumento na estimativa para as tensões máximas quando se enriquecem os campos de tensão e deslocamento no domínio.

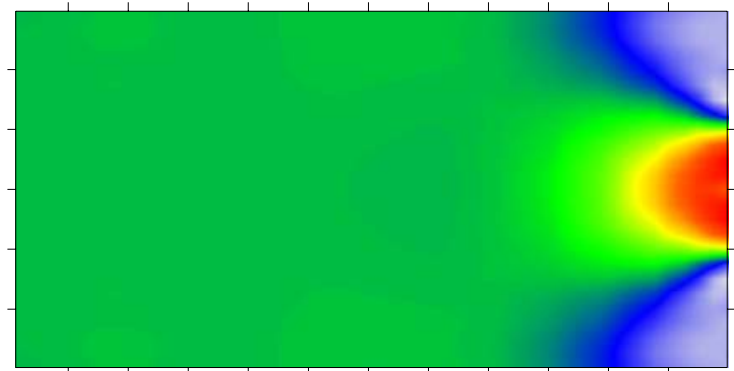

Sigma-X

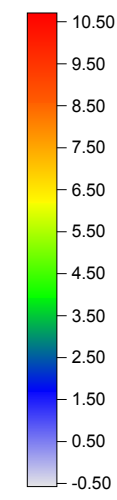

Sigma-y
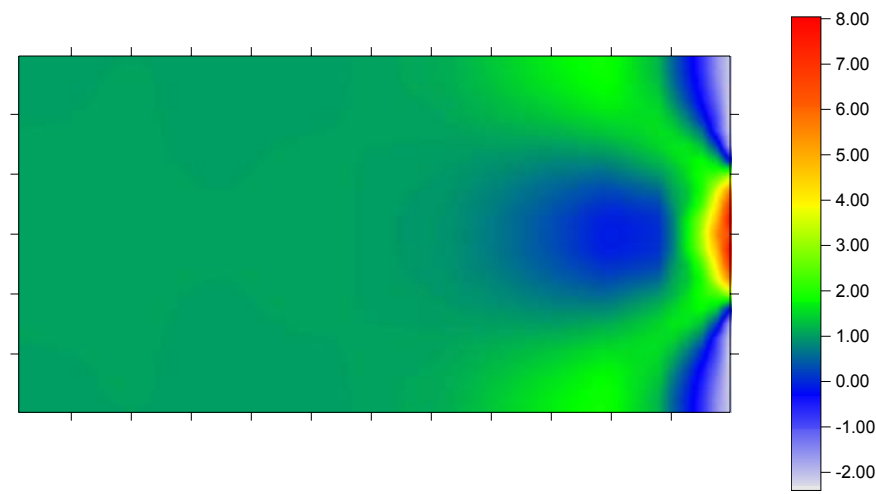

$-2.00$

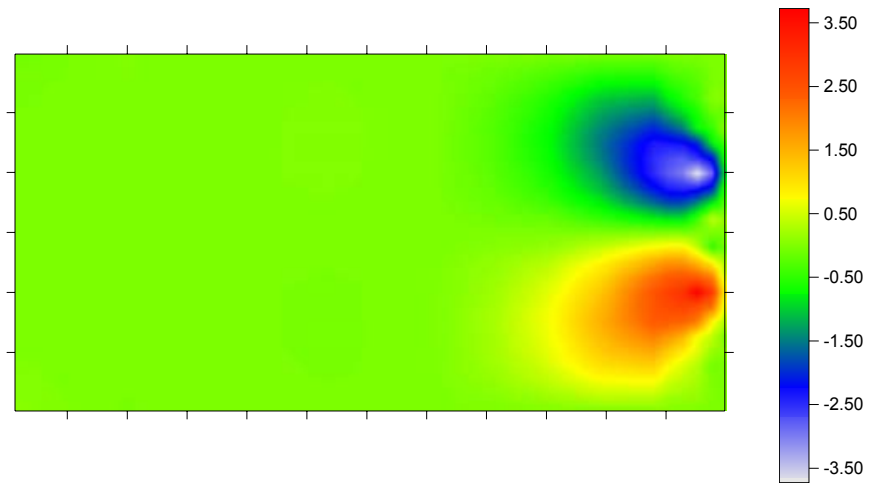

Tau-xy

Figura 7.16 - Representação do campo de tensões com enriquecimento do tipo $\left(y-y_{n \dot{\alpha}}\right)^{2}+\left(x-x_{n \dot{\delta}}\right)^{2}+\left(y-y_{n \dot{\delta}}\right)\left(x-x_{n \dot{\alpha}}\right)$ sobre $\sigma \quad$ e $u$ no $\Omega$ dezesseis nós $(64,65,77,78,90,91,103,104,116,117,129,130,142,143,155$ e 156 ) e em $u$ no $\Gamma$ - seis nós $(78,91,104,117,130$ e 143$)$.

A figura 7.16 mostra que o enriquecimento simultâneo sobre os campos de domínio e contorno também proporciona um aumento na estimativa dos campos de tensão.

- Resultados para malha regular $12 \times 15$ - enriquecimento polinomial por função de Airy com graus de enriquecimento $d_{e}=1$ e $d_{e}=2$.

Nas tabelas 7.8 e 7.9 mostram os resultados gerais dos ensaios numéricos realizados com a possibilidade de enriquecimento das bases aproximativas dos campos de tensão no domínio com funções de Airy descritas por polinômios. 
Tabela 7.8 - Resultados gerais obtidos para o problema 1 com malha regular $12 \times 15 \mathrm{e}$ função enriquecedora de Airy do tipo polinomial $\left(d_{e}=1\right)$.

\begin{tabular}{ccc}
\hline \multirow{2}{*}{ Condições de Enriquecimento } & Energia de Deformação & \multirow{2}{*}{ Teste do Mosaico } \\
\cline { 2 - 3 } Sem enriquecimento & $\boldsymbol{U}_{E M}=46,47$ & Ok! \\
\hline Todos os nós enriquecidos $-\sigma-\Omega$ & 46,52 & Ok! \\
\hline Dezesseis nós enriquecidos $-\sigma-\Omega$ & 46,12 & Ok! \\
\hline
\end{tabular}

Na tabela 7.8 utiliza-se o grau de enriquecimento $\boldsymbol{d}_{e}=1$. Já os resultados do tabela 7.9 correspondem a um grau de enriquecimento $\boldsymbol{d}_{e}=2$. Além do enriquecimento seletivo (ver figura 7.13) utiliza-se o enriquecimento em todos os nós do domínio para efeito de comparação.

Tabela 7.9 - Resultados gerais obtidos para o problema 1 com malha regular $12 \times 15 \mathrm{e}$ função de Airy do tipo polinomial $\left(d_{e}=2\right)$.

\begin{tabular}{ccc}
\hline Condições de Enriquecimento & Energia de Deformação & \multirow{2}{*}{ Teste do Mosaico } \\
\cline { 2 - 3 } Sem enriquecimento & $\boldsymbol{U}_{E M}=46,47$ & Ok! \\
\hline Todos os nós enriquecidos $-\sigma-\Omega$ & 46,52 & Ok! \\
\hline Dezesseis nós enriquecidos $-\sigma-\Omega$ & 43,06 & Ok! \\
\hline
\end{tabular}

Para enriquecimento com $\boldsymbol{d}_{\boldsymbol{e}}=\boldsymbol{1}$ as estimativas das energias de deformação estão muito próximas as obtidas com enriquecimento polinomial. Observa-se que para $\boldsymbol{d}_{\boldsymbol{e}}=\mathbf{2}$ as estimativas da energia de deformação são menores que as obtidas para $\boldsymbol{d}_{\boldsymbol{e}}=1$ e da energia de deformação do exemplo modelo.

$\mathrm{Na}$ figura 7.17, destacam-se as representações do campo de tensões com enriquecimento com $\boldsymbol{d}_{e}=\mathbf{2}$ para a condição de dezesseis nós enriquecidos no domínio.

Ainda na figura 7.17, verifica-se uma maior estimativa dos valores máximos, comparando-se com a situação sem enriquecimento (ver figura 7.14). 


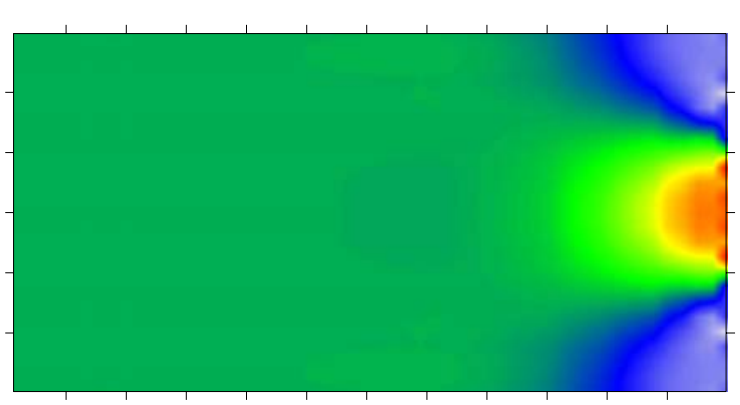

Sigma-X

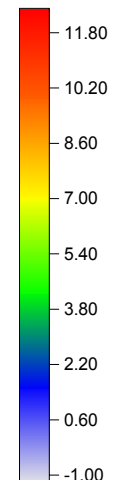

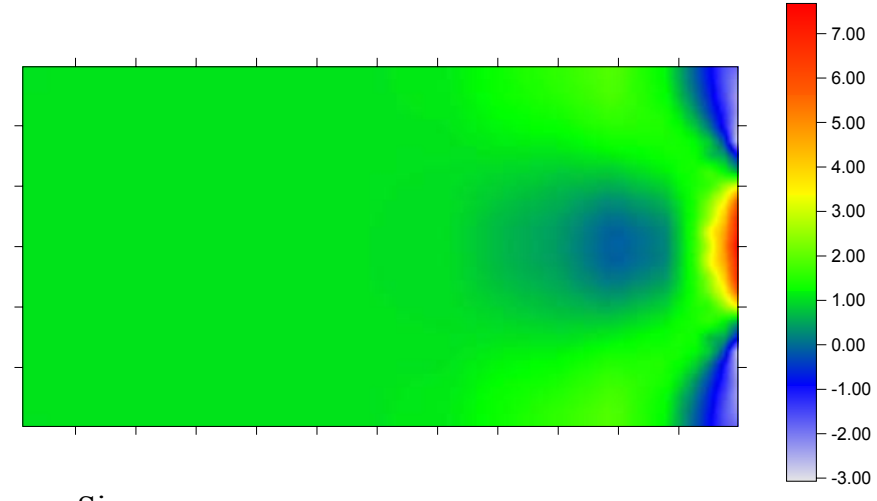

Sigma-y

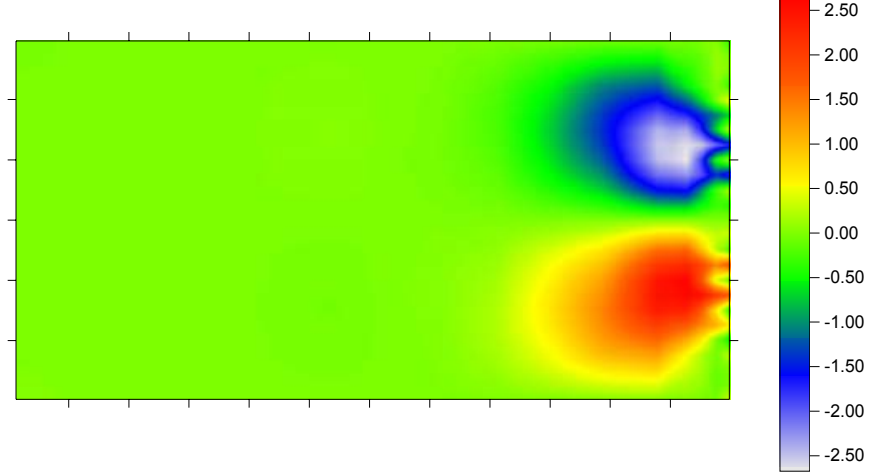

Tau-xy

Figura 7.17- Representação do campo de tensões com enriquecimento do tipo $\boldsymbol{d}_{\boldsymbol{e}}=\mathbf{2}$ dezesseis nós sobre $\sigma(64,65,77,78,90,91,103,104,116,117,129$, $130,142,143,155$ e 156$)$.

- Resultados para malha regular 12x15 - enriquecimento com funções trigonométricas.

Finalizando as análises do problema 1, apresentam-se alguns resultados de ensaios numéricos realizados com enriquecimentos, utilizando funções trigonométricas (ver tabela 7.10). Como funções enriquecedoras adotadam-se $\operatorname{sen}\left\lfloor\left(y-y_{n \dot{b}}\right)^{2}\right\rfloor \mathrm{e}$ $\boldsymbol{c o s}\left\lfloor\left(\boldsymbol{x}-\boldsymbol{x}_{\boldsymbol{n} \boldsymbol{\delta}}\right)^{2}\right\rfloor$ para enriquecimento dos campos de domínio e $\operatorname{sen}\left\lfloor\left(\boldsymbol{y}-\boldsymbol{y}_{\boldsymbol{n} \boldsymbol{\delta}}\right)^{2}\right\rfloor$ para o enriquecimento do deslocamento no contorno.

Da tabela 7.10, verifica-se que, para todas as condições de enriquecimento apresentadas o valor da energia de deformação ficou bem próxima à energia de referência do exemplo modelo. 
Tabela 7.10 - Resultados gerais obtidos para o problema 1 com malha irregular $12 \times 15$ e o enriquecimento realizado com funções trigonométricas.

\begin{tabular}{|c|c|c|}
\hline \multirow{2}{*}{ Condições de Enriquecimento } & Energia de Deformação & \multirow{2}{*}{$\begin{array}{l}\text { Teste do } \\
\text { Mosaico }\end{array}$} \\
\hline & $U_{E M}=46,47$ & \\
\hline Sem enriquecimento & 46,25 & Ok! \\
\hline Todos os nós enriquecidos $-\sigma$ e $u-\Omega-\operatorname{sen}\left[\left(y-y_{n o}\right)^{2}\right]$ & 46,17 & Ok! \\
\hline Todos os nós enriquecidos $-\sigma-\Omega-\operatorname{sen}\left[\left(y-y_{n o}\right)^{2}\right]$ & 46,06 & Ok! \\
\hline Dezesseis nós enriquecidos - $\sigma$ e $u-\Omega \operatorname{sen}\left[\left(y-y_{n}\right)^{2}\right]$ & 46,17 & Ok! \\
\hline Dezesseis nós enriquecidos $\left.-\sigma-\Omega-\operatorname{sen}\left[\left(y-y_{n}\right)^{2}\right)^{2}\right]$ & 46,11 & Ok! \\
\hline Todos os nós enriquecidos - $\sigma$ e $u-\Omega-\cos \left[\left(x-x_{n \dot{o}}\right)^{2}\right]$ & 46,14 & Ok! \\
\hline Todos os nós enriquecidos $-\sigma-\Omega-\cos \left[\left(x-x_{n o}\right)^{2}\right]$ & 45,46 & Ok! \\
\hline Dezesseis nós enriquecidos - $\sigma$ e $\left.u-\Omega-\cos \left[\left(x-x_{n o}\right)^{2}\right]\right)$ & 46,25 & Ok! \\
\hline Dezesseis nós enriquecidos $-\sigma-\Omega-\cos \left[\left(x-x_{n o}\right)^{2}\right]$ & 46,23 & Ok! \\
\hline $\begin{array}{c}\left.\text { Dezesseis nós enriquecidos - } \sigma \text { e } u-\Omega-\operatorname{sen}\left[\left(y-y_{n o}\right)^{2}\right]\right) \mathrm{e} \\
\text { seis nós }-u-\Gamma-\operatorname{sen}\left[\left(y-y_{n \dot{\prime}}\right)^{2}\right]\end{array}$ & 46,29 & Ok! \\
\hline
\end{tabular}

No tocante aos campos de deslocamentos, há uma maior compatibilidade entre os deslocamentos de domínio e contorno quando se enriquece somente o campo de tensões. Em geral, os deslocamentos do nó de referência de contorno (208) ( $\Gamma$ ) estão próximos aos do exemplo modelo. Ver tabela 7.11.

Tabela 7.11 - Deslocamentos do nó de referência da malha de cobertura de domínio e contorno para a malha irregular $12 \times 15$.

\begin{tabular}{|c|c|c|}
\hline \multirow{3}{*}{ Condições de Enriquecimento } & \multicolumn{2}{|c|}{ Deslocamentos do nó de referência } \\
\hline & \multicolumn{2}{|c|}{$u_{x E M_{2}}=0,2818$ e $u_{y E M_{2}}=0,0000$} \\
\hline & Deslocamento do nó $208(\Gamma)$ & Deslocamento do nó $208(\Omega)$ \\
\hline Sem enriquecimento & $\boldsymbol{u}_{x}=0,2868$ e $u_{y}=0,0000$ & $u_{x}=0,2885$ e $u_{y}=-0,0119$ \\
\hline $\begin{array}{l}\text { Todos os nós enriquecidos - } \sigma-\Omega- \\
\operatorname{sen}\left[\left(y-y_{\text {nó }}\right)^{2}\right]\end{array}$ & $\boldsymbol{u}_{\boldsymbol{x}}=0,3013$ e $u_{y}=0,0000$ & $u_{x}=0,3007 \mathrm{e} u_{y}=0,0005$ \\
\hline $\begin{array}{l}\text { Dezesseis nós enriquecidos }-\sigma \text { e } u- \\
\Omega-\operatorname{sen}\left[\left(y-y_{n o ́}\right)^{2}\right]\end{array}$ & $u_{x}=0,2980$ e $u_{y}=0,0000$ & $u_{x}=0,3007$ e $u_{y}=-0,0050$ \\
\hline $\begin{array}{l}\text { Dezesseis nós enriquecidos - } \sigma-\Omega \text { - } \\
\operatorname{sen}\left[\left(y-y_{\text {nó }}\right)^{2}\right]\end{array}$ & $\boldsymbol{u}_{x}=0,30129$ e $u_{y}=0,0000$ & $u_{x}=0,30414$ e $u_{y}=-0,0038$ \\
\hline $\begin{array}{l}\text { Dezesseis nós enriquecidos - } \sigma-\Omega \text { - } \\
\cos \left[\left(x-x_{n o}\right)^{2}\right]\end{array}$ & $\boldsymbol{u}_{\boldsymbol{x}}=0,2867$ e $u_{y}=0,0000$ & $u_{x}=0,2906 \mathrm{e} u_{y}=-0,0142$ \\
\hline $\begin{array}{l}\text { Dezesseis nós enriquecidos - } \sigma \text { e } u- \\
\left.\Omega-\operatorname{sen}\left[\left(y-y_{n o ́}\right)^{2}\right]\right) \text { e seis nós }-u-\Gamma- \\
\operatorname{sen}\left[\left(y-y_{n o ́}\right)^{2}\right]\end{array}$ & $\boldsymbol{u}_{x}=0,30168$ e $u_{y}=0,0000$ & $u_{x}=0,2955 \mathrm{e} u_{y}=0,0087$ \\
\hline
\end{tabular}

Nas figuras 7.18 a 7.20 apresentam-se os campos de tensões para algumas das condições de enriquecimento abordadas na tabela 7.10. 

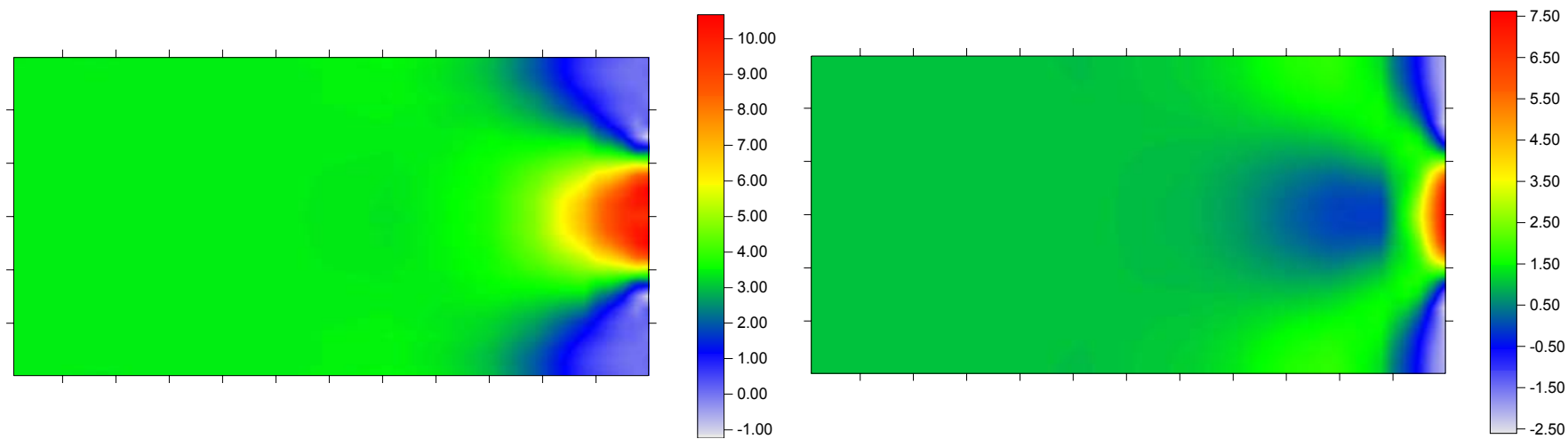

Sigma-x

Sigma-y
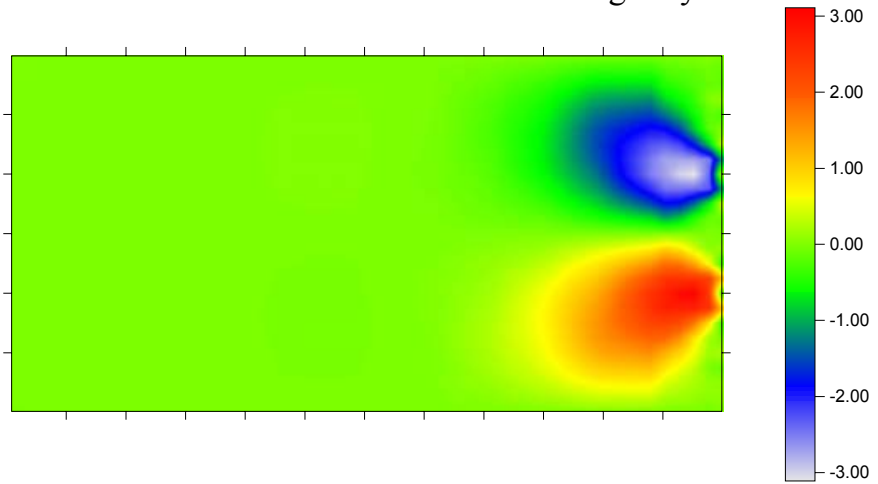

Tau-xy

Figura 7.18- Representação do campo de tensões com enriquecimento do tipo sen $\left\lfloor\left(y-y_{n \dot{b}}\right)^{2}\right\rfloor$ sobre $\sigma$ e $u$ no $\Omega$ - todos os nós.

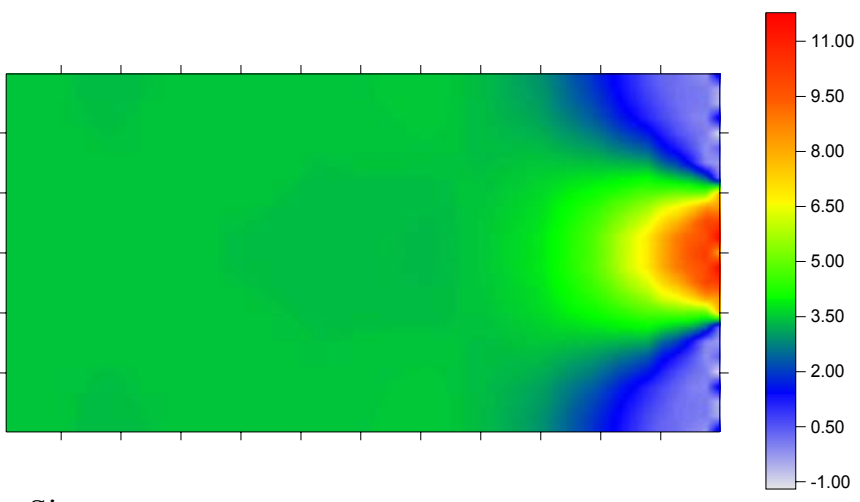

Sigma $-\mathrm{x}$

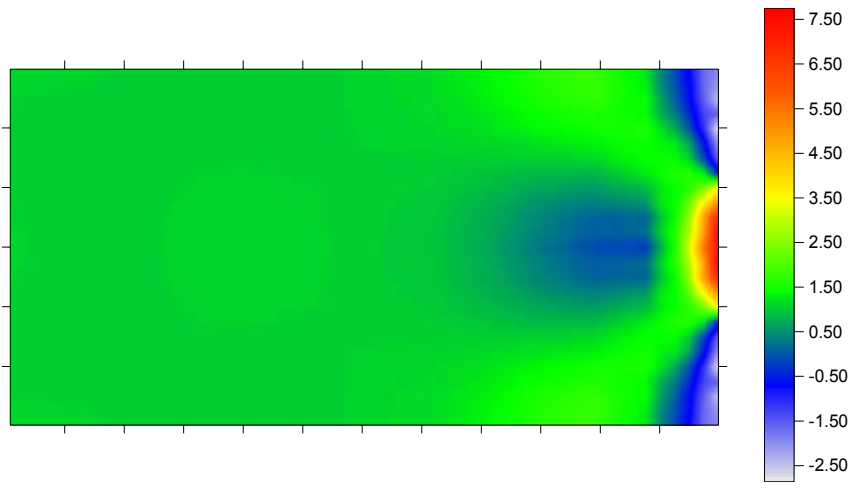

Sigma-y

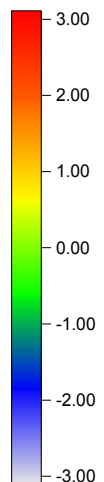

Tau-xy

Figura 7.19- Representação do campo de tensões com enriquecimento do tipo sen $\left.\mid\left(y-y_{n \dot{b}}\right)^{2}\right\rfloor$ sobre $\sigma$ no $\Omega$ - todos os nós. 
A figura 7.18 mostra que o campo de tensão na direção x (Sigma-x) apresentase mais regular com o de enriquecimento lá indicado. Da figura 7.19, observa-se uma melhor representação do efeito da concentração da tensão Sigma-x na região de descontinuidade (limite da região de aplicação do carregamento nas cotas de 20 e 40 unidades de comprimento do lado vertical esquerdo da chapa).

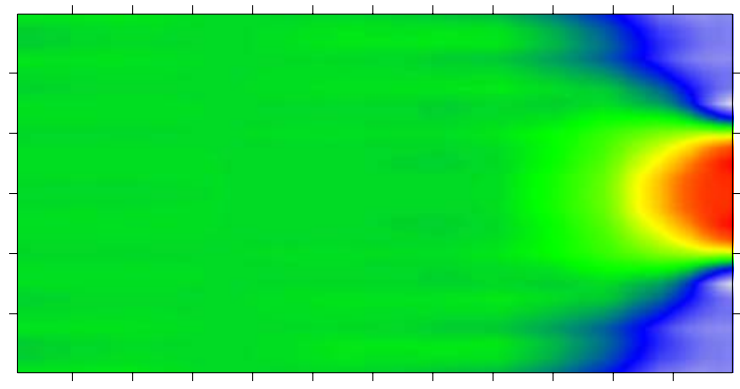

Sigma-x

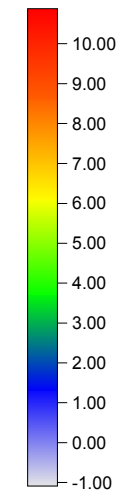

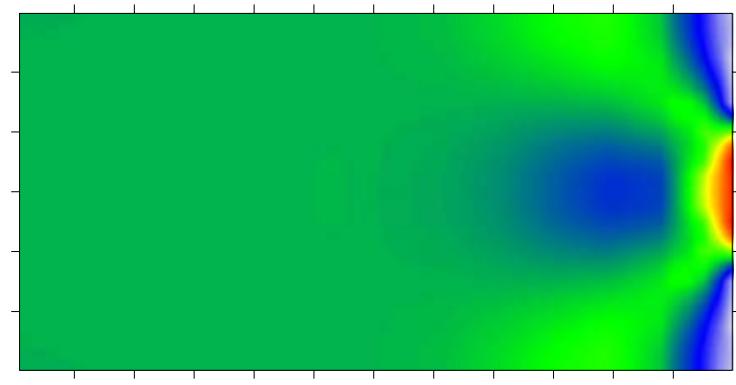

Sigma-y

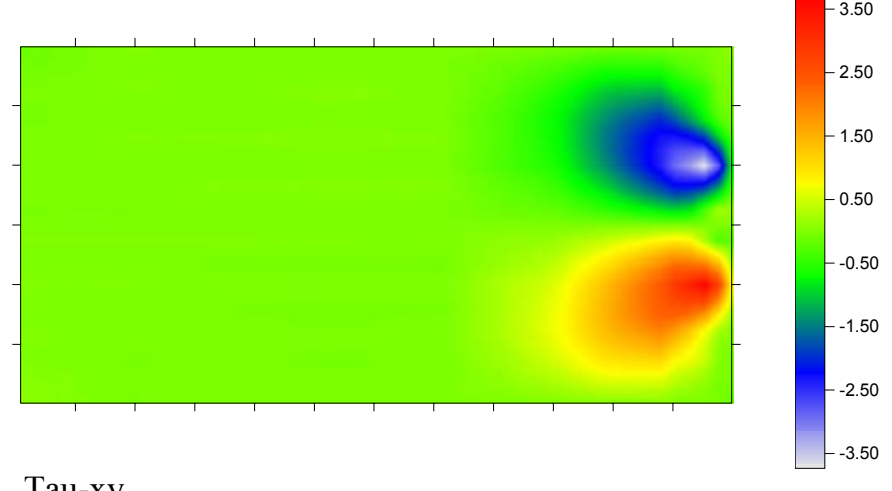

Tau-xy

Figura 7.20- Representação dos campo de tensões com enriquecimento do tipo $\cos \left[\left(\boldsymbol{x}-\boldsymbol{x}_{\boldsymbol{n}}\right)^{2}\right]$ sobre $\sigma$ e $u$ no $\Omega$ - dezesseis nós $(64,65,77,78,90$, $91,103,104,116,117,129,130,142,143,155$ e 156 ).

Com o enriquecimento do tipo $\cos \left\lfloor\left(\boldsymbol{x}-\boldsymbol{x}_{\boldsymbol{n}}\right)^{2}\right\rfloor$ em dezesseis nós das bases aproximativas do domínio, como apresentado na figura 7.20, não há muitas mudanças quando se compara com os campos de tensão obtido da situação sem enriquecimento. 


\subsection{Problema 2: Chapa com Fenda}

Para a geração do conjunto de resultados de comparação do problema 2, com o ANSYS $^{\circledR}$ adotou-se a malha representada na figura 7.21 com um total de 1648 elementos triangulares.

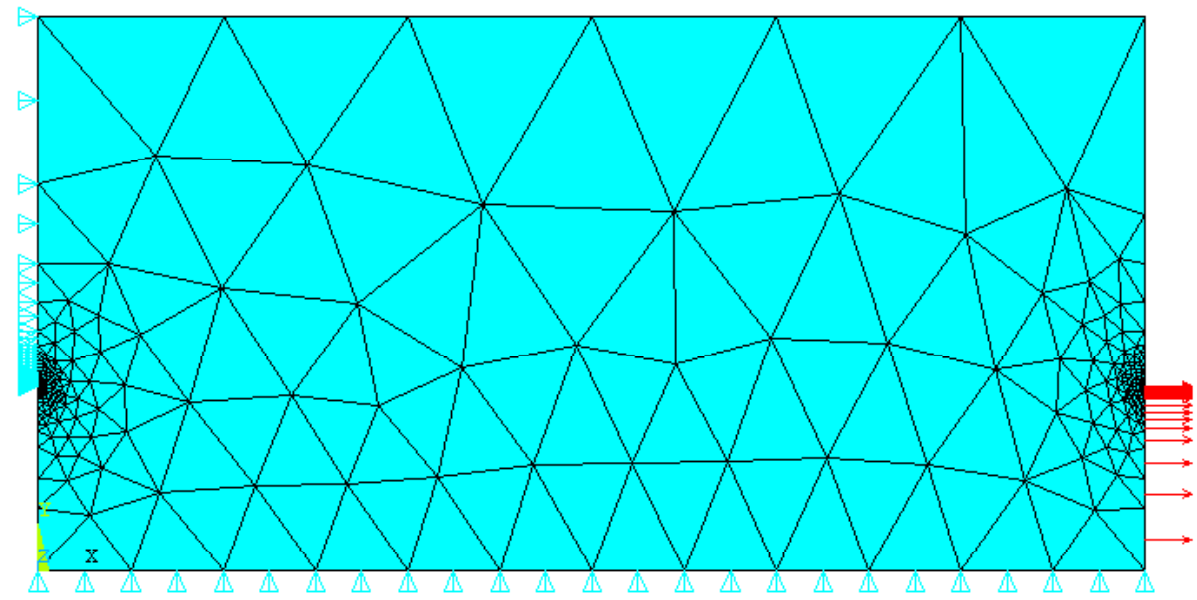

Figura 7.21 - Exemplo modelo.

Nessa discretização, observa-se um maior refinamento na vizinhança da ponta da fenda e próximo de um dos limites da zona de aplicação do carregamento. O elemento triangular da biblioteca do ANSYS ${ }^{\circledR}$ utilizado na análise é o mesmo do exemplo modelo do problema 1. Como valor da energia de deformação, obteve-se: 65,98 . O valor exato da energia de deformação do problema 2 para a geometria e carregamento adotado é: 66,01. No tocante aos deslocamentos de referência, o correspondentes ao nó do canto superior direito, obtiveram-se como resultados: $\boldsymbol{u}_{\boldsymbol{x}}=0,2125$ e $\boldsymbol{u}_{\boldsymbol{y}}=0,0667$.

A visualização da resposta para o campo de tensão próximo à ponta da fenda é apresentada na figura 7.22. Nota-se, claramente, a concentração de tensão próxima a essa região.

Para análise da FHMT com enriquecimento nodal, duas malhas de elementos são selecionadas: a primeira é regular e bastante simples com três divisões na direção $\boldsymbol{x}$ e três na direção $\boldsymbol{y}$, enquanto que a segunda é irregular com doze divisões na direção $\boldsymbol{x}$ e nove na direção $\boldsymbol{y}$. A figura 7.23 apresenta a geometria das discretizações adotadas.

No problema 2, analogamente ao problema 1, qualquer nó da malha de cobertura de domínio pode ser enriquecido. Já os enriquecimentos dos nós da malha de cobertura de contorno são realizados somente nos lados verticais (direito e esquerdo) e no lado horizontal superior da chapa, onde não existem condições de contorno essenciais 
prescritas. A aplicação do Teste do Mosaico, segue todas as recomendações adotadas no problema 1.

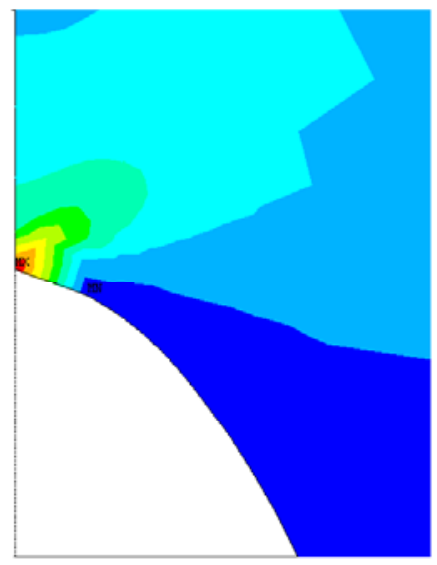

Sigma-X

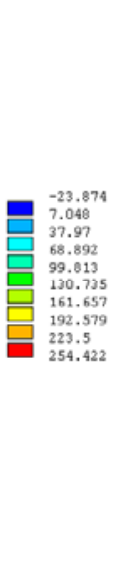

Sigma-y

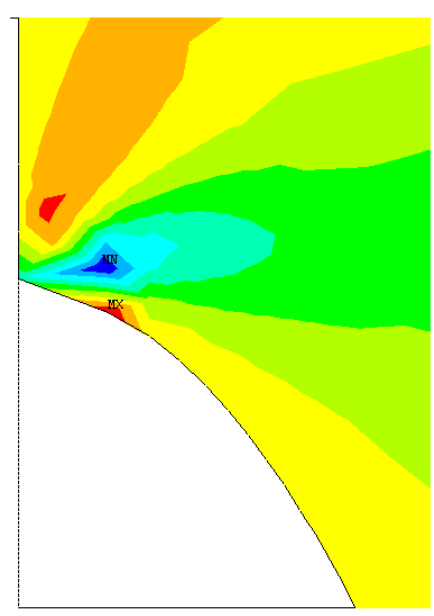

Tau-xy

Figura 7.22 - Exemplo modelo - representação do campo de tensões próximo à ponta da fenda.

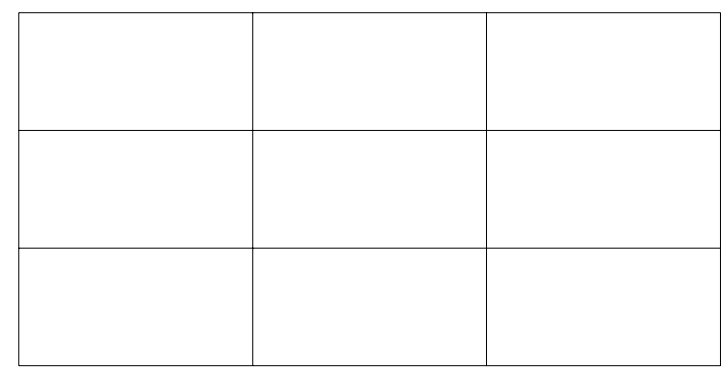

Malha regular $3 \times 3$

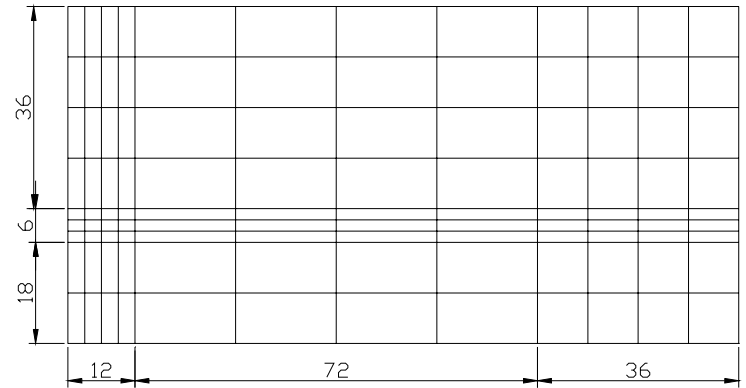

Malha irregular 12x9

Figura 7.23 - Discretizações adotadas para análise do problema 2.

Apresentam-se, no que segue, os resultados gerais (representação dos campos de tensão, deslocamentos do nó de referência considerando-o como nó de domínio e de contorno, energia de deformação e verificação do Teste do Mosaico), para as discretizações adotadas com algumas situações de enriquecimento.

Para leitura da tabela de resultados, considera-se a seguinte legenda:

- $\Omega$ : domínio;

- $\Gamma$ : contorno;

- $\sigma$ : aproximação do campo de tensão;

- $u$ : aproximação do campo de deslocamento;

- Energia de deformação do exemplo modelo: $\boldsymbol{U}_{E M}=65,98$; 
- Energia de deformação exata do problema 2: $\boldsymbol{U}_{\text {exatoP } 2}=66,01$;

- Deslocamento de referência dos exemplos modelos: $\boldsymbol{u}_{x E M}=0,2125$ e $\boldsymbol{u}_{\boldsymbol{y E M}}=0,0667$

- Resultados para malha regular 3x3 - enriquecimento polinomial com a função: $\left(\boldsymbol{y}-\boldsymbol{y}_{\boldsymbol{n} \boldsymbol{\delta}}\right)^{2}$ no domínio e no contorno.

Os resultados apresentados colocam em confronto as possibilidades de enriquecimento do total do conjunto de nós ou de alguns deles. No caso de enriquecimento seletivo, os nós selecionados estão na região da aplicação do carregamento e da ponta da fenda. Os seis nós enriquecidos na malha de cobertura de domínio, utilizados nos ensaios da tabela 7.12, são: 1, 2, 5, 6, 9 e 10. O nó da malha de contorno enriquecido foi o nó 8 . O nó de referência para os campos de deslocamento é o nó 16 (canto superior direito). Tal conjunto de nós está ilustrado na figura 7.24.

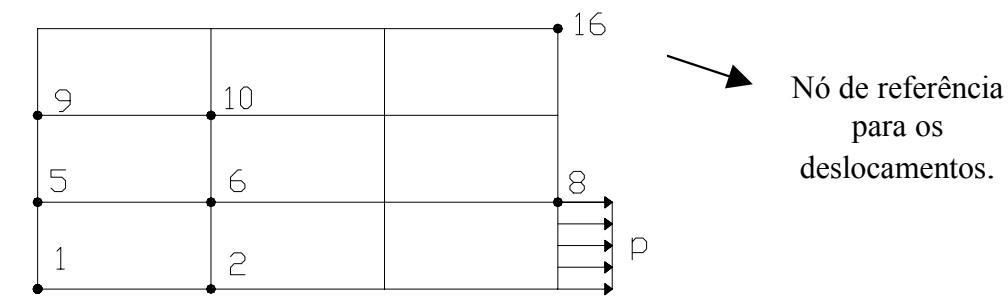

Figura 7.24 - Nós da malha regular 3x3 escolhidos no enriquecimento seletivo.

Mesmo com uma malha pobre, os resultados da tabela 7.12 mostram que a energia de deformação para cada situação de enriquecimento, onde se verifica o Teste do Mosaico, está próxima ao valor exato da energia de deformação do problema 2.

No entanto, como a malha regular 3x3 é uma malha muito pobre, não é possível colher o efeito da concentração de tensões na ponta da fenda, conforme mostra a figura 7.25 . 
Tabela 7.12 - Resultados gerais do problema 2 obtidos com a malha regular 3x3 para algumas situações com enriquecimento polinomial.

\begin{tabular}{ccc}
\hline & Energia de Deformação & \\
\cline { 2 - 3 } Condição de Enriquecimento & Teste do Mosaico \\
& $U_{\text {exato } 2}=66,01 \boldsymbol{U}_{\boldsymbol{E} M}=\mathbf{6 5 , 9 8}$ & \\
\hline Sem enriquecimento & 67,63 & Ok! \\
\hline Todos os nós enriquecidos $-\sigma$ e $u-\Omega$ & 62,06 & Ok! \\
\hline Todos os nós enriquecidos $-\sigma-\Omega$ & 59,45 & Ok! \\
\hline Todos os nós enriquecidos $-u-\Omega$ & Não convergiu & Ok! \\
\hline Seis nós enriquecidos $-\sigma$ e $u$ no $\Omega$ & 68,08 & Ok! \\
\hline Seis nós enriquecidos $-\sigma$ no $\Omega$ & 66,01 & Okão Ok! \\
\hline Seis nós enriquecidos $-u$ no $\Omega$ & Não convergiu & Ok! \\
\hline $\begin{array}{c}\text { Seis nós enriquecidos }-\sigma \text { e } u-\Omega \text { e } \\
\text { um nó enriquecido }-u-\Gamma\end{array}$ & 70,11 & Não Ok! \\
\hline $\begin{array}{c}\text { Seis nós enriquecidos }-\sigma-\Omega \text { e } \\
\text { um nó enriquecido }-u-\Gamma\end{array}$ & 67,28 & \\
\hline $\begin{array}{c}\text { Seis nós enriquecidos }-u-\Omega \text { e } \\
\text { um nó no contorno }-u-\Gamma\end{array}$ & Não convergiu & \\
\hline
\end{tabular}

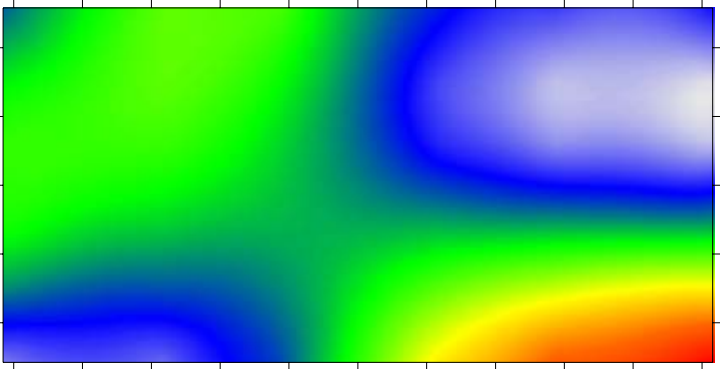

Sigma-x sem enriquecimento
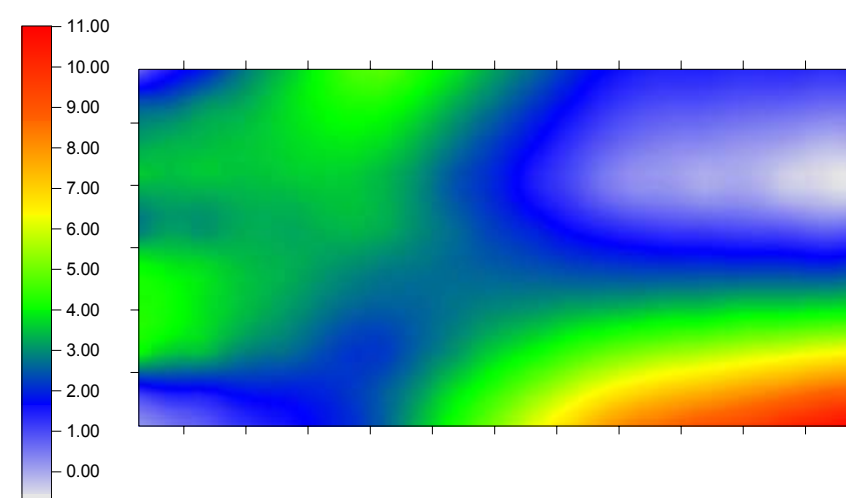

Sigma-x com enriquecimento em seis nós em $\Omega$

Figura 7.25 - Representação do campo de tensão Sigma $-\mathrm{x}$ sem e com enriquecimento em seis nós $\sigma$ no $\Omega$.

- Resultados para malha regular 3x3 - Enriquecimento com funções que se assemelham às soluções da Mecânica da Fratura sobre cada componente do campo aproximado das tensões.

O objetivo maior do enriquecimento sobre as aproximações dos campos de tensões, com as eq.(5.37), eq. (5.38) e eq.(5.39), é a possibilidade de capturar o efeito da concentração de tensões próximo à região da ponta da fissura. Enriquecendo-se somente o campo das tensões em quatro nós do domínio (5, 6, 9 e 10), já é possível capturar esse efeito mesmo com a malha regular $3 \times 3$, como ilustra a figura 7.26. 


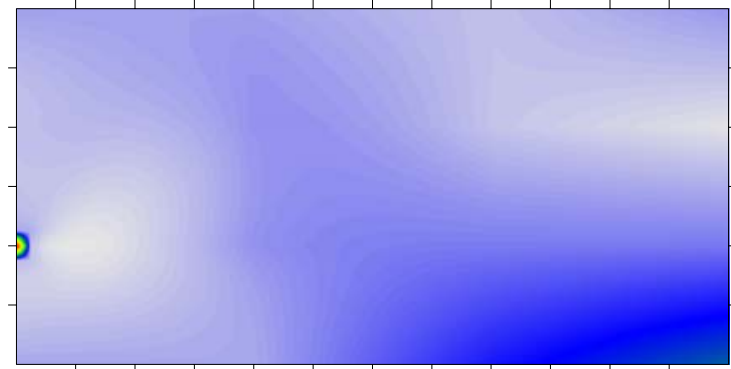

Sigma-X

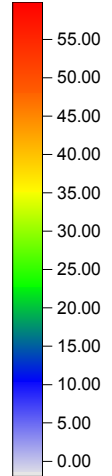

Sigma-y

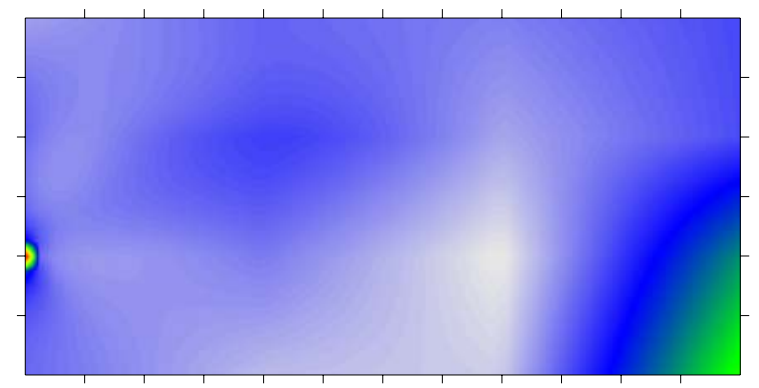

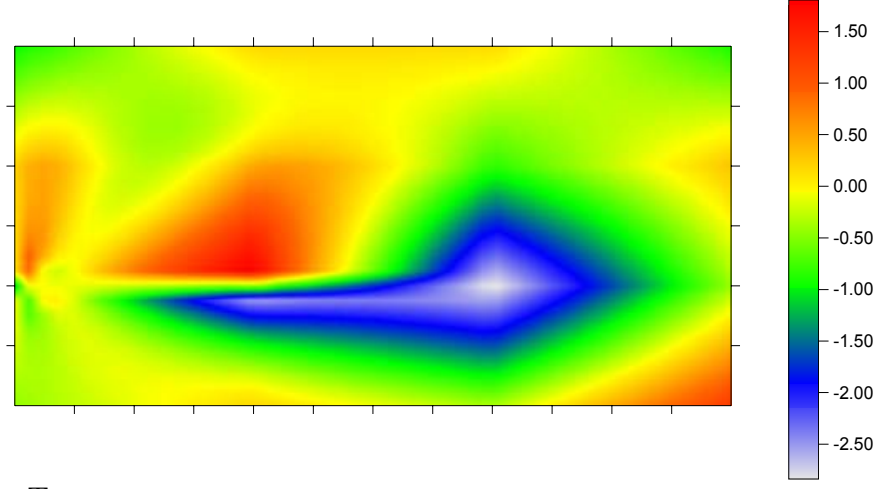

Tau-xy

Figura 7.26 - Representação do campo de tensões com enriquecimento por meio de funções que se assemelham à solução singular da Mecânica da Fratura.

Já em termos de valores numéricos, em razão da malha adotada, resultam energias de deformação bem menores que as de referência, conforme mostram os dados da tabela 7.13 .

Tabela 7.13 - Resultados gerais do problema 2 obtidos com a malha regular $3 \times 3$ para algumas situações com enriquecimento não polinomial.

\begin{tabular}{|c|c|c|}
\hline \multirow[b]{2}{*}{ Condição de Enriquecimento } & Energia de Deformação & \multirow[b]{2}{*}{ Teste do Mosaico } \\
\hline & $U_{\text {exatoP } 2}=66,01 U_{E M}=\mathbf{6 5 , 9 8}$ & \\
\hline Sem enriquecimento & 67,63 & Ok! \\
\hline Seis nós enriquecidos $-\sigma$ no $\Omega$ & 50,14 & Ok! \\
\hline Quatro nós $(5,6,9$ e 10$)$ enriquecidos - $\sigma$ no $\Omega$ & 50,30 & Ok! \\
\hline
\end{tabular}

- Resultados para malha irregular 12x9 - enriquecimento polinomial com a função: $\left(y-y_{n \dot{o}}\right)^{2}+\left(x-x_{n \dot{o}}\right)^{2}+\left(y-y_{n \dot{o}}\right)\left(x-x_{n \dot{ }}\right)$ no domínio $\mathrm{e}$ $\left(y-y_{n \dot{o}}\right)^{2}$ no contorno.

Os resultados deste caso também se referem a um enriquecimento do conjunto total ou seletivo dos nós da malha. Para visualização dessa segunda opção, na figura 
7.27 estão representados os quatorze nós enriquecidos no domínio $(12,13,26,27,28$, $38,39,40,41,53,54,66,67)$ e os três enriquecidos no contorno $(27,26$ e 39). O nó de referência para efeito de comparação entre os campos de deslocamento é o nó 130.

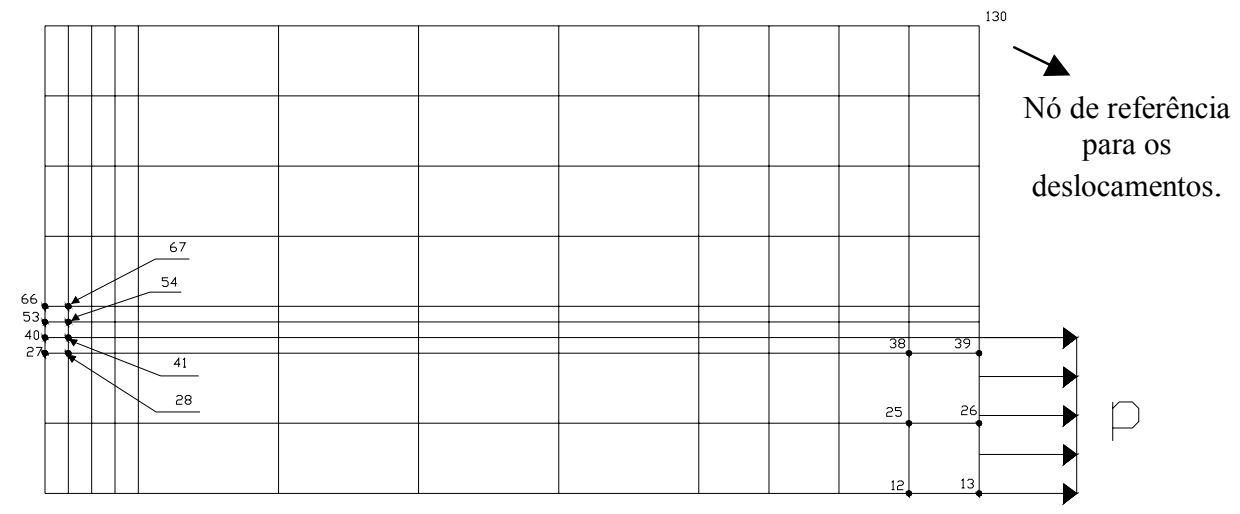

Figura 7.27 - Nós da malha irregular 12x9 selecionados para o enriquecimento.

Tabela 7.14 - Resultados gerais do problema 2 obtidos com a malha irregular 12x9 para algumas situações com enriquecimento polinomial.

\begin{tabular}{|c|c|c|}
\hline \multirow[b]{2}{*}{ Condição de Enriquecimento } & Energia de Deformação & \multirow[b]{2}{*}{ Teste do Mosaico } \\
\hline & $U_{\text {exatoP } 2}=66,01 U_{E M}=\mathbf{6 5 , 9 8}$ & \\
\hline Sem enriquecimento & 62,05 & Ok! \\
\hline Todos os nós enriquecidos $-\sigma$ e $u-\Omega$ & 61,20 & Ok! \\
\hline Todos os nós enriquecidos $-\sigma-\Omega$ & 59,58 & Ok! \\
\hline Todos os nós enriquecidos $-u-\Omega$ & Não convergiu & Não Ok! \\
\hline Quatorze nós enriquecidos - $\sigma-\Omega$ & 61,02 & Ok! \\
\hline $\begin{array}{c}\text { Quatorze nós enriquecidos }-\sigma-\Omega \mathrm{e} \\
\text { Três nós enriquecidos }-u-\Gamma\end{array}$ & 61,45 & Ok! \\
\hline $\begin{array}{l}\text { Quatorze nós enriquecidos - } \sigma \text { e } u-\Omega \text { e } \\
\text { Três nós enriquecidos }-u-\Gamma\end{array}$ & 61,93 & Ok! \\
\hline
\end{tabular}

A energia de deformação resultante da análise com a malha irregular 12x9, para a situação sem enriquecimento, está um pouco abaixo da energia de deformação exata do problema e da energia de deformação obtida com a malha regular 3x3, como mostra a tabela 7.14. Os enriquecimentos realizados sobre o campo de deslocamentos no contorno fornecem as maiores energias de deformação, dentre as situações com enriquecimento.

Os deslocamentos do nó de referência (130) são apresentados na tabela 7.15.

Os deslocamentos do nó de referência da malha de cobertura de contorno, em todos os casos de enriquecimento seletivo mostrado na tabela 7.15, aproximam-se dos deslocamentos de referência. Em particular, o enriquecimento sobre os campos 
aproximados no domínio favorece a compatibilidade entre os campos de deslocamento do domínio e contorno.

Tabela 7.15 - Deslocamentos do nó de referência da malha de cobertura de domínio e contorno para a malha irregular $12 \times 9$ com enriquecimento polinomial.

\begin{tabular}{|c|c|c|}
\hline \multirow{3}{*}{ Condição de Enriquecimento } & \multicolumn{2}{|c|}{ Deslocamentos do nó de referência } \\
\hline & \multicolumn{2}{|c|}{$u_{x E M}=0,2125 \mathrm{e} u_{y E M}=0,0667$} \\
\hline & Deslocamento do nó $130(\Gamma)$ & Deslocamento do nó $130(\Omega)$ \\
\hline Sem enriquecimento & $u_{x}=0,2648$ e $u_{y}=0,0612$ & $u_{x}=0,1039$ e $u_{y}=0,2229$ \\
\hline Todos os nós enriquecidos - $\sigma$ e $u-\Omega$ & $u_{x}=0,2327$ e $u_{y}=0,0645$ & $u_{x}=0,0304 \mathrm{e} u_{y}=-0,0760$ \\
\hline Todos os nós enriquecidos - $\sigma-\Omega$ & $u_{x}=0,2345$ e $u_{y}=0,0560$ & $u_{x}=0,2347$ e $u_{y}=0,0551$ \\
\hline Quatorze nós enriquecidos - $\sigma-\Omega$ & $u_{x}=0,2370$ e $u_{y}=0,0677$ & $u_{x}=0,2370$ e $u_{y}=0,0692$ \\
\hline $\begin{array}{l}\text { Quatorze nós enriquecidos }-\sigma-\Omega \mathrm{e} \\
\text { Três nós enriquecidos }-u-\Gamma\end{array}$ & $u_{x}=0,2350$ e $u_{y}=0,0691$ & $u_{x}=0,2393 \mathrm{e} u_{y}=0,0707$ \\
\hline $\begin{array}{l}\text { Quatorze nós enriquecidos - } \sigma \text { e } u-\Omega \\
\text { e Três nós enriquecidos }-u-\Gamma\end{array}$ & $u_{x}=0,2390$ e $u_{y}=0,0659$ & $u_{x}=0,2434 \mathrm{e} u_{y}=0,0623$ \\
\hline
\end{tabular}

Quanto aos campos de tensões, representadas na figura 7.28, com a malha irregular 12x9 sem enriquecimento já é possível observar o efeito da concentração de tensões na região da ponta da fissura, mesmo com uma imprecisão quanto à posição da zona de concentração, que deveria estar mais próxima à cota de 20 unidades de comprimento.

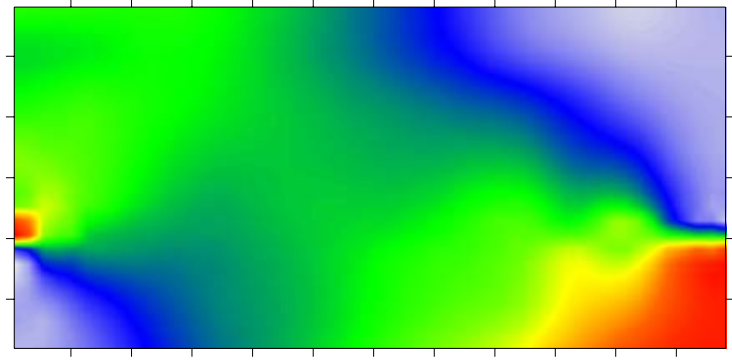

Sigma-x

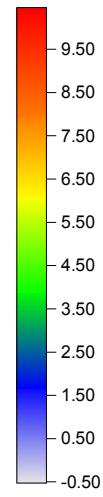

$-0.50$

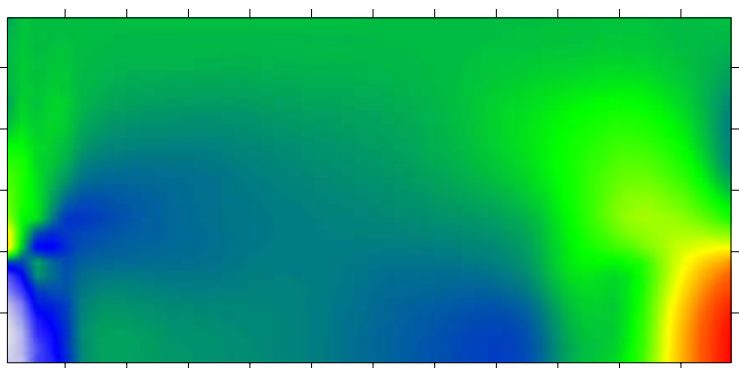

Sigma-y

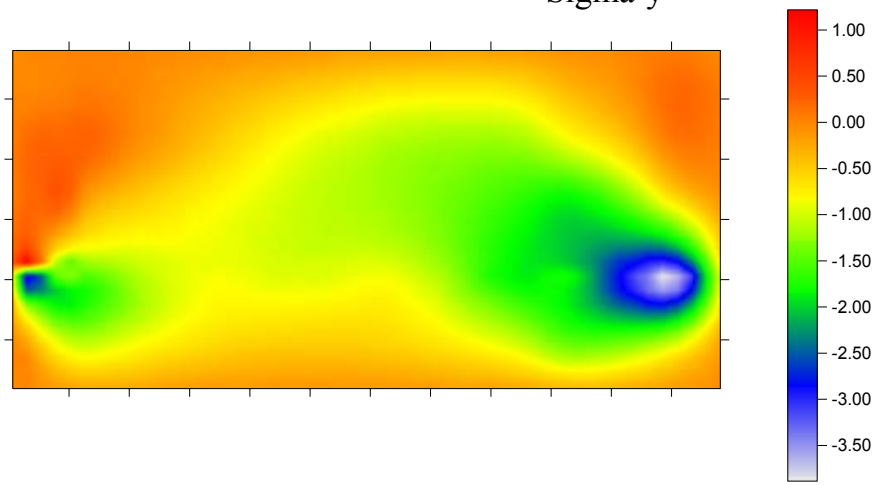

Tau-xy

Figura 7.28 - Representação do campo de tensões sem enriquecimento. 
As figuras 7.29 e 7.30 apresentam os campos de tensões com enriquecimento em todos os nós sobre o campo de tensão no domínio e sobre os campos de tensão e deslocamento no domínio, respectivamente.

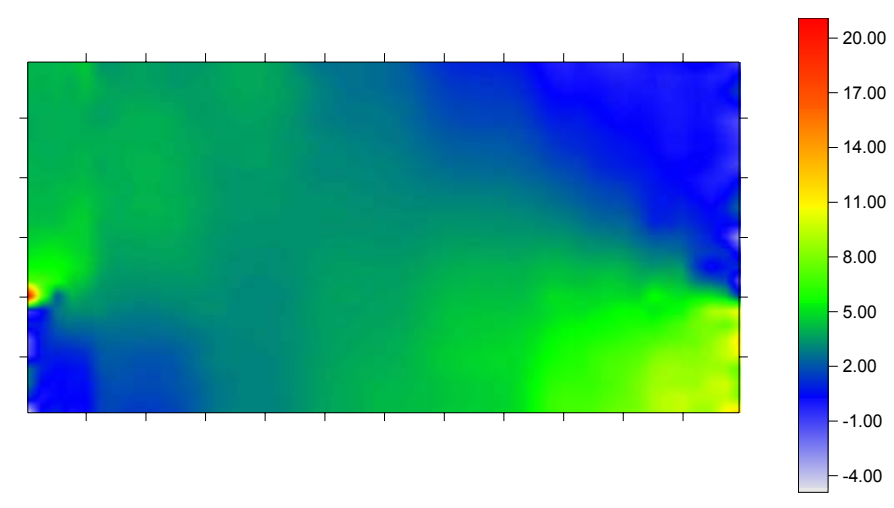

Sigma-X

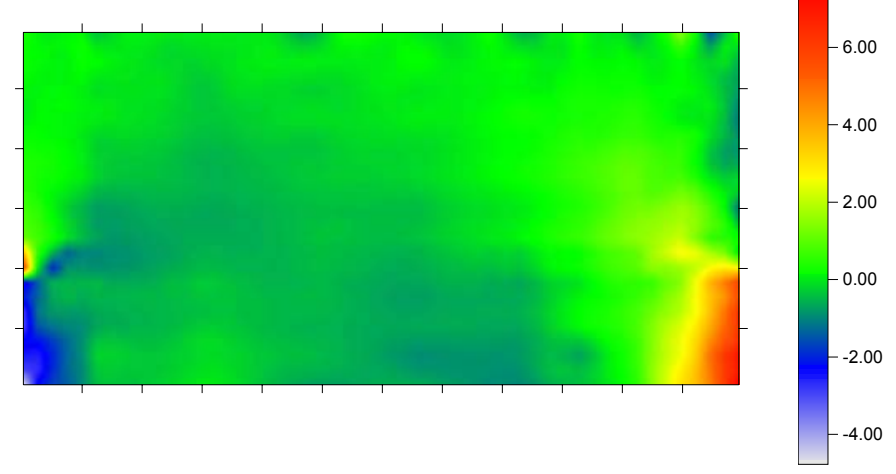

Sigma-y

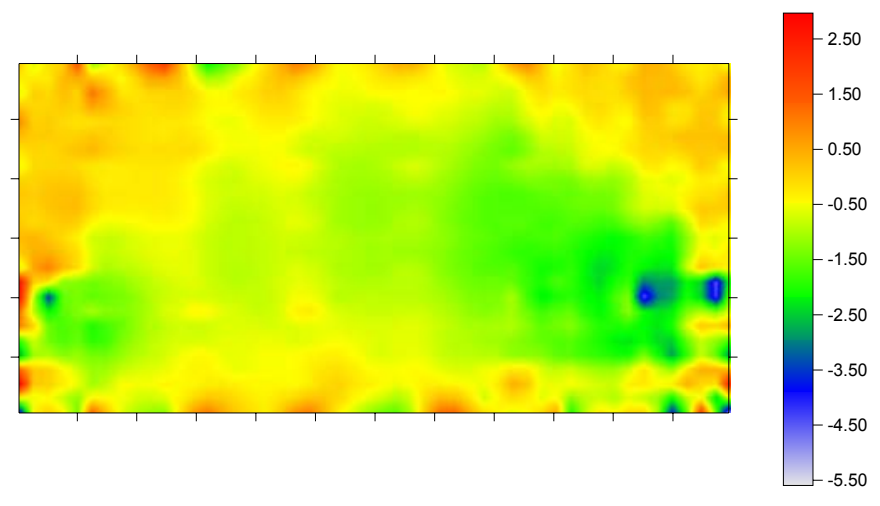

Tau-xy

Figura 7.29 -Representação do campo de tensões com enriquecimento do tipo $\left(y-y_{n \dot{o}}\right)^{2}+\left(x-x_{n \dot{o}}\right)^{2}+\left(y-y_{n \dot{o}}\right)\left(x-x_{n \dot{b}}\right)$ sobre $\sigma$ em todos os nós do $\Omega$.

Nota-se do confronto das figuras 7.29 e 7.30 com a 7.28, que com esses tipos de enriquecimento é possível uma melhor reprodução do efeito de concentração de tensões na aponta da fenda. Ainda entre as figura 7.29 e 7.30, nota-se que o enriquecimento de ambos os campos aproximados no domínio proporciona uma maior regularidade dos campos de tensões. 


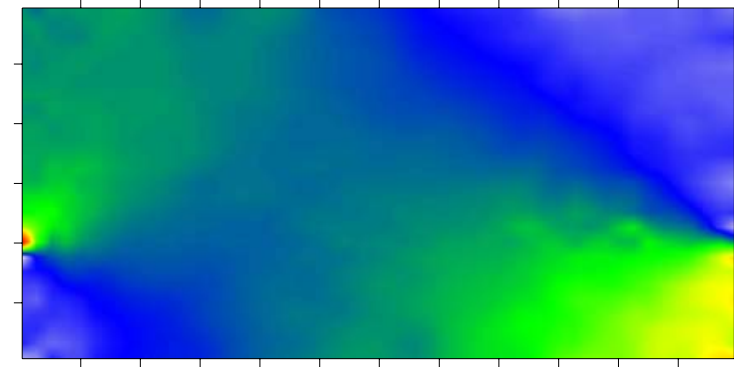

Sigma-X

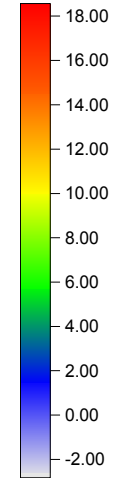

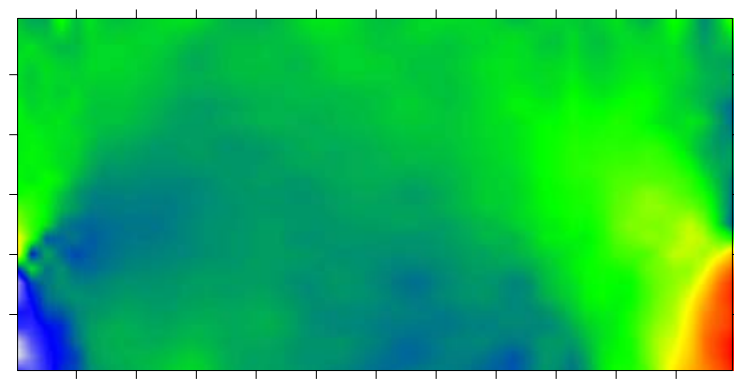

Sigma-y

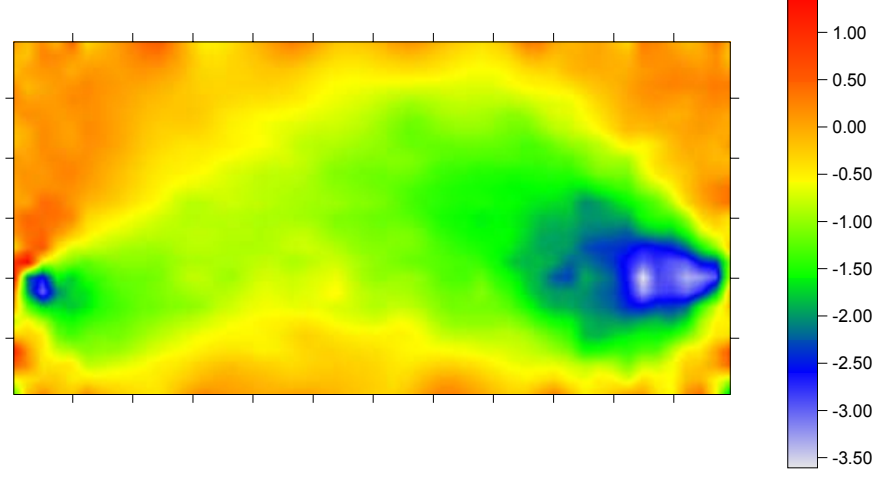

Tau-xy

Figura 7.30- Representação do campo de tensões com enriquecimento do tipo $\left(y-y_{n \dot{ }}\right)^{2}+\left(x-x_{n \dot{ }}\right)^{2}+\left(y-y_{n \dot{ }}\right)\left(x-x_{n \dot{ }}\right)$ sobre $\sigma$ e $u$ em todos os nós do $\Omega$.

- Respostas para malha irregular 12x9 - Enriquecimento com funções que se assemelham às soluções da Mecânica da Fratura sobre cada componente do campo aproximado das tensões no domínio (sigma-x, sigma-y e tau-xy) e sobre cada componente do campo de deslocamento no domínio $\left(u_{x}\right.$ e $\left.u_{y}\right)$.

O enriquecimento das aproximações dos campos do domínio com as eq.(5.37), eq. (5.38), eq.(5.39), eq.(5.40) e eq.(5.41), não proporcionam uma queda na estimativa da energia de deformação, como foi observado na análise semelhante realizada com a malha regular3x3. Conseqüentemente, os deslocamentos do nó de referência da malha de cobertura de contorno ficam próximos aos dos exemplos modelos.

Nas figuras 7.31 e 7.32 são apresentadas as visualizações dos campos de tensão para as situações de enriquecimento descritas na tabela 7.16. Observa-se que a zona de concentração de tensões ficou bem definida já com poucos nós enriquecidos em torno da ponta da fissura. 
Tabela 7.16 - Resultados gerais do problema 2 obtidos com a malha irregular 12x9 para algumas situações com enriquecimento não polinomial.

\begin{tabular}{|c|c|c|}
\hline \multirow{2}{*}{ Condições de Enriquecimento } & Energia de Deformação & \multirow{2}{*}{ Teste do Mosaico } \\
\hline & $U_{\text {exatoP } 2}=66,01 \quad U_{E M}=\mathbf{6 5 , 9 8}$ & \\
\hline Sem enriquecimento & 62,05 & Ok! \\
\hline $\begin{array}{c}\text { Quatro nós }(40,41,53 \text { e } 54) \text { enriquecidos }-\sigma \text { no } \\
\Omega\end{array}$ & 61,02 & Ok! \\
\hline $\begin{array}{c}\text { Seis nós }(26,39,40,41,53 \text { e } 54) \text { enriquecidos - } \sigma \text { e } \\
u \text { nо } \Omega\end{array}$ & 61,43 & Ok! \\
\hline
\end{tabular}

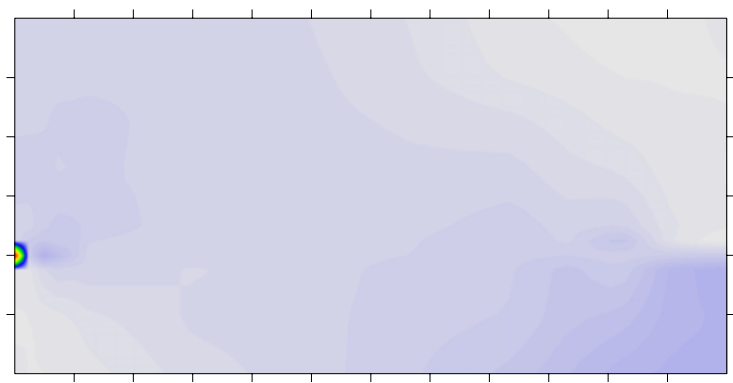

Sigma- $\mathrm{x}$
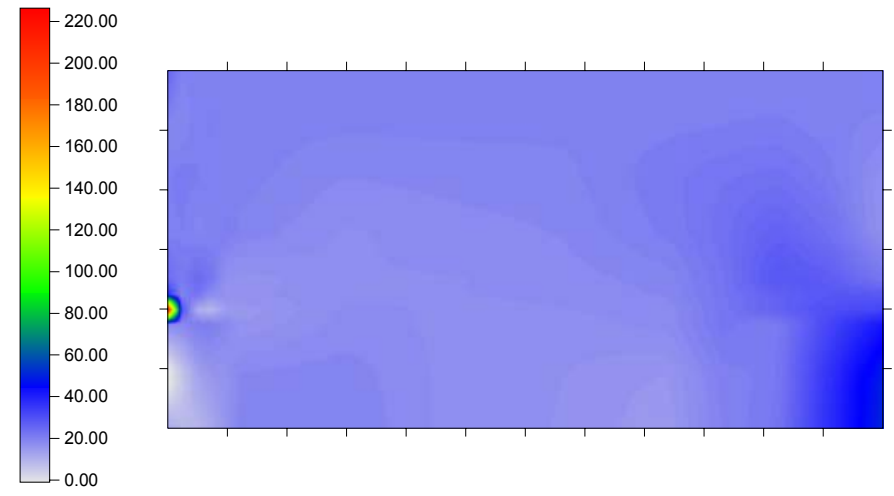

Sigma-y

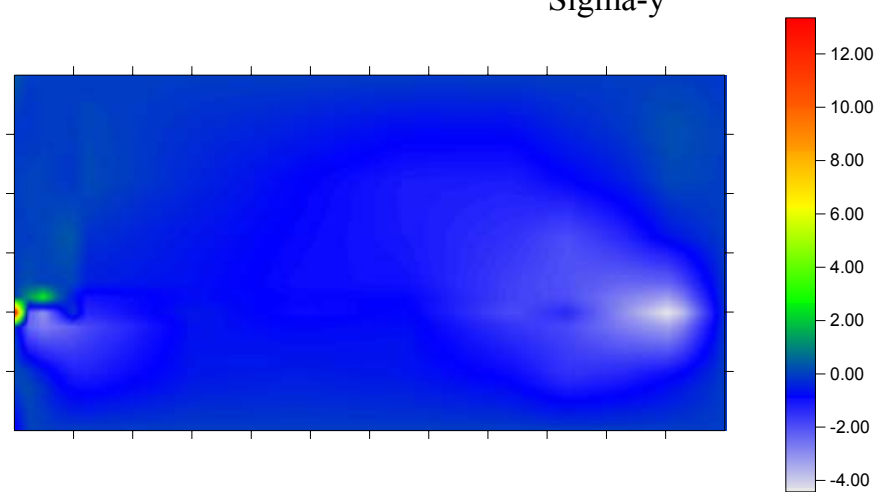

Tau-xy

Figura 7.31 - Representação do campo de tensões com enriquecimento por meio de funções que se assemelham à solução singular da Mecânica da Fratura sobre $\sigma$ - quatro nós $(40,41,53$ e 54$)$. 


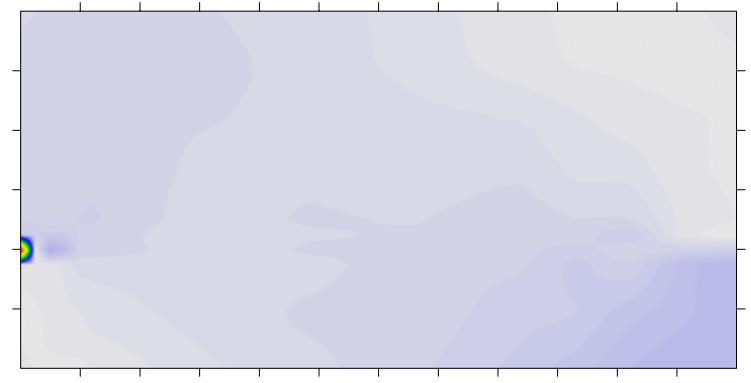

Sigma $-\mathrm{x}$
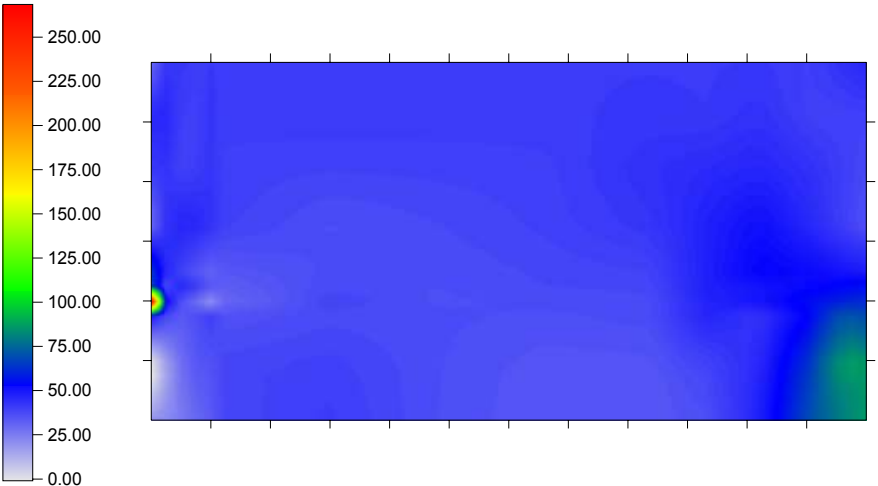

Sigma-y

Tau-xy

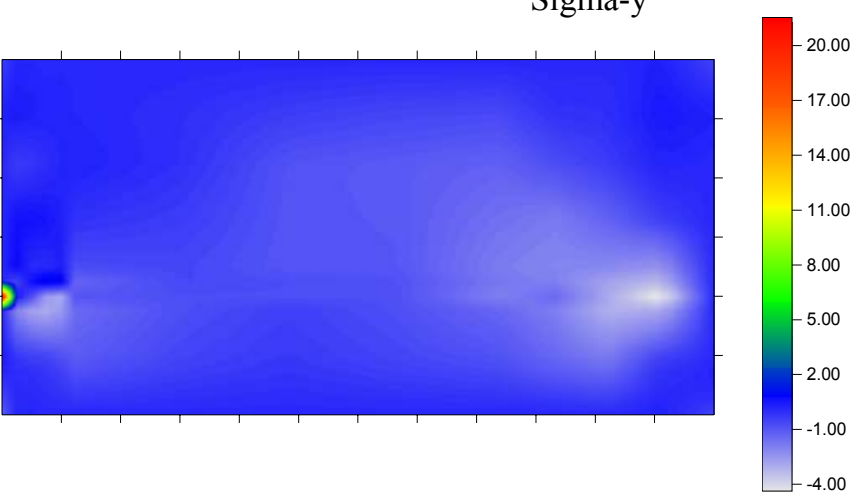

20.00

17.00

4.00

1.00

.00

.00

4.00

Figura 7.32 - Representação do campo de tensões com enriquecimento por meio de funções que se assemelham à solução singular da Mecânica da Fratura sobre $\sigma$ e $u$ - seis nós $(26,39,40,41,53$ e 54$)$.

- Respostas para malha irregular 12x9 - enriquecimento com funções de Airy considerando o grau de enriquecimento $d_{e}=1$ e $\boldsymbol{d}_{e}=2$.

Nas tabelas 7.17 e 7.18 mostram os resultados gerais dos ensaios numéricos realizados com a possibilidade de enriquecimento das bases aproximativas dos campos de tensão no domínio com os tensões auto-equilibrados.

Tabela 7.17 - Resultados gerais obtidos para o problema 2 com malha irregular $12 \mathrm{x} 9 \mathrm{e}$ função de Airy enriquecedora do tipo polinomial $\left(d_{e}=1\right)$.

\begin{tabular}{|c|c|c|}
\hline \multirow{2}{*}{ Condição de Enriquecimento } & Energia de Deformação & \multirow{2}{*}{ Teste do Mosaico } \\
\hline & $U_{\text {exatoP2 }}=66,01 \quad U_{E M}=65,98$ & \\
\hline Sem enriquecimento & 62,05 & Ok! \\
\hline Todos os nós enriquecidos $-\sigma-\Omega$ & 61,14 & Ok! \\
\hline Quatorze nós enriquecidos - $\sigma-\Omega$ & 61,77 & Ok! \\
\hline
\end{tabular}

Na tabela 7.17, para as condições de enriquecimento apresentadas, foi utilizado o grau de enriquecimento $\boldsymbol{d}_{\boldsymbol{e}}=\mathbf{1}$; já os resultados do tabela 7.18 foram obtidos com o 
grau de enriquecimento $\boldsymbol{d}_{e}=2$. Além dos nós propostos para enriquecimento seletivo (ver figura 7.27) utilizou-se do enriquecimento em todos os nós do para efeito de comparação.

Tabela 7.18 - Resultados gerais obtidos para o problema 2 com malha irregular $12 \times 9$ e função de Airy enriquecedora do tipo polinomial $\left(\boldsymbol{d}_{e}=2\right)$.

\begin{tabular}{|c|c|c|}
\hline \multirow{2}{*}{ Condição de Enriquecimento } & Energia de Deformação & \multirow{2}{*}{ Teste do Mosaico } \\
\hline & $U_{\text {exatoP } 2}=66,01 \quad U_{E M}=65,98$ & \\
\hline Sem enriquecimento & 62,05 & Ok! \\
\hline Todos os nós enriquecidos $-\sigma-\Omega$ & 57,34 & Ok! \\
\hline Quatorze nós enriquecidos - $\sigma-\Omega$ & 60,57 & Ok! \\
\hline
\end{tabular}

Observa-se que as energias de deformação para essas situações de enriquecimento foram muito parecidas com as obtidas com outros tipos de enriquecimento. As representações dos campos de tensão (ver figuras 7.33 e 7.34) e os valores de deslocamentos (ver tabela 7.19) também tiveram um comportamento semelhante quando do enriquecimento por funções polinomiais. Com os dois graus de enriquecimento $\left(\boldsymbol{d}_{e}=\mathbf{1}\right.$ e $\boldsymbol{d}_{e}=2$ ), conseguiu-se uma melhor definição da zona de concentração de tensões na cota de 20 unidades de comprimento, como apresentam as figuras 7.33 e 7.34 .

Tabela 7.19 - Deslocamentos do nó de referência da malha de cobertura de domínio e contorno para a malha irregular $12 \times 9$ e função de Airy enriquecedora do tipo polinomial $\left(\boldsymbol{d}_{e}=2\right)$.

\begin{tabular}{ccc}
\hline \multirow{2}{*}{ Condição de Enriquecimento } & \multicolumn{2}{c}{ Deslocamentos do nó de referência } \\
\cline { 2 - 3 } & \multicolumn{2}{c}{$\boldsymbol{u}_{\boldsymbol{x} E M}=0,2125 \mathrm{e} \boldsymbol{u}_{\boldsymbol{y} M}=0,0667$} \\
\cline { 2 - 3 } & Deslocamento do nó $\mathbf{1 3 0}(\Gamma)$ & Deslocamento do nó 130 $(\Omega)$ \\
\hline Sem enriquecimento & $u_{x}=0,2648 \mathrm{e} u_{y}=0,0612$ & $u_{x}=0,1039$ e $u_{y}=0,2229$ \\
\hline Todos os nós enriquecidos $-\sigma-\Omega$ & $\boldsymbol{u}_{\boldsymbol{x}}=\mathbf{0 , 2 5 0 1}$ e $\boldsymbol{u}_{\boldsymbol{y}}=\mathbf{0 , 0 3 4 2}$ & $\boldsymbol{u}_{\boldsymbol{x}}=\mathbf{0 , 2 4 9 9}$ e $\boldsymbol{u}_{\boldsymbol{y}}=\mathbf{0 , 0 3 4 6}$ \\
\hline Quatorze nós enriquecidos $-\sigma-\Omega$ & $\boldsymbol{u}_{\boldsymbol{x}}=\mathbf{0 , 2 3 1 4}$ e $\boldsymbol{u}_{\boldsymbol{y}}=\mathbf{0 , 0 6 6 9}$ & $\boldsymbol{u}_{\boldsymbol{x}}=\mathbf{0 , 2 3 0 7}$ e $\boldsymbol{u}_{\boldsymbol{y}}=\mathbf{0 , 0 7 1 6}$ \\
\hline
\end{tabular}




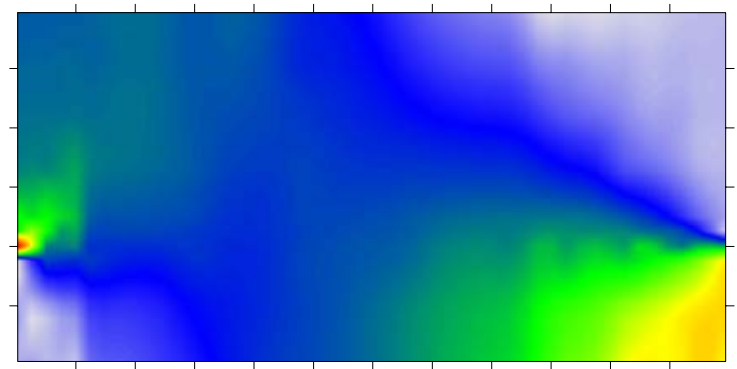

Sigma-x
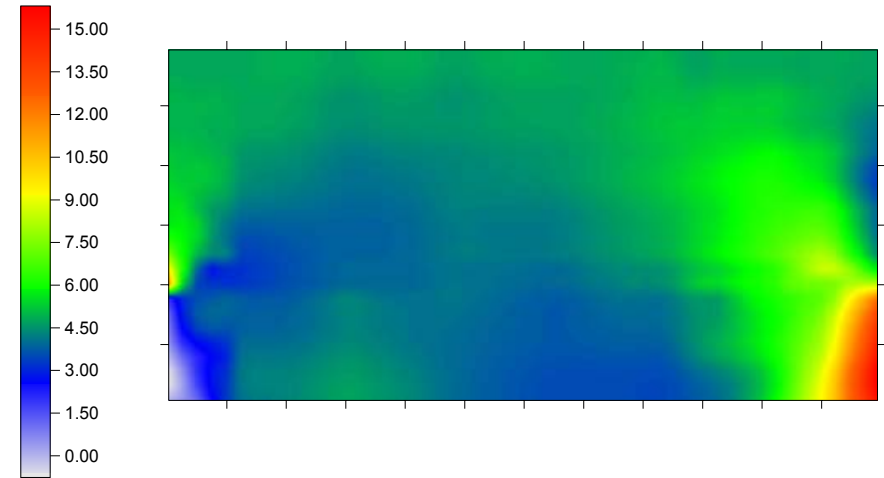

Sigma-y

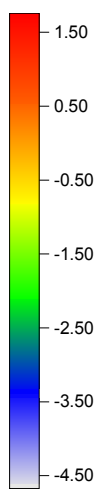

Tau-xy

Figura 7.33- Representação do campo de tensões com enriquecimento do tipo $\boldsymbol{d}_{\boldsymbol{e}}=\mathbf{1}$ sobre $\sigma$ em todos os nós .

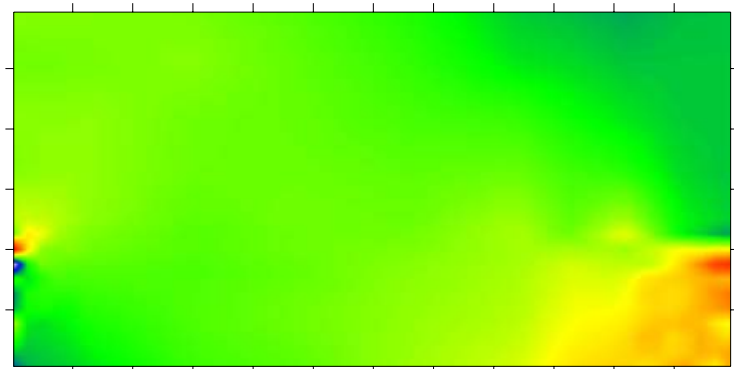

Sigma-x

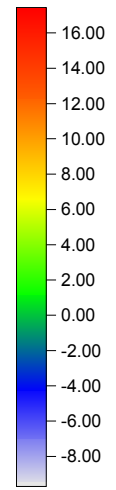

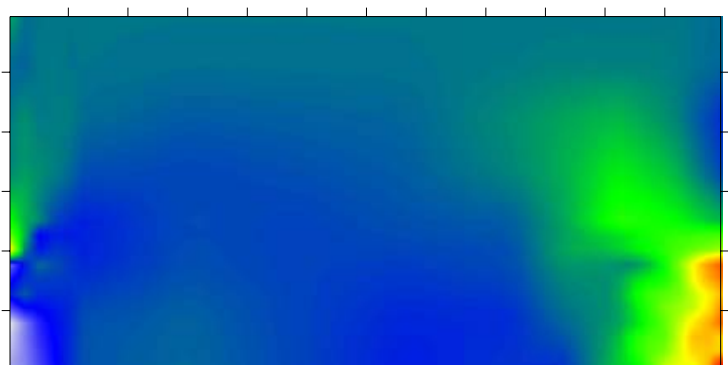

Sigma-y

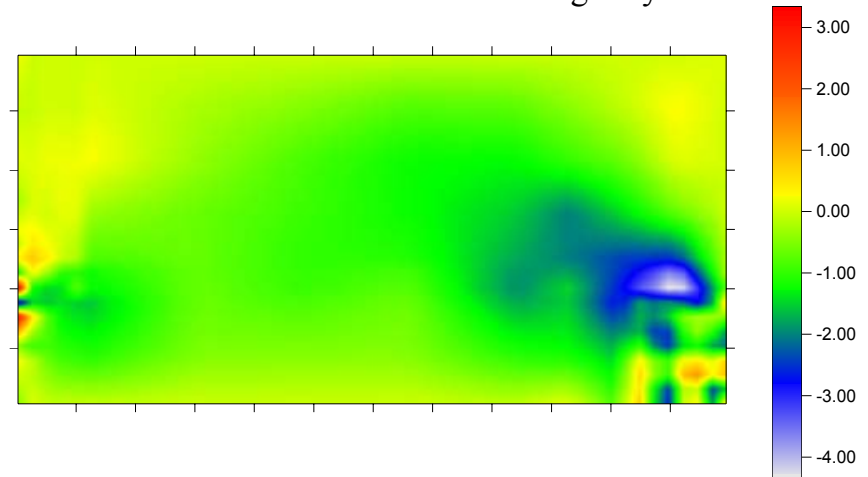

Tau-xy

Figura 7.34- Representação do campo de tensões com enriquecimento do tipo $\boldsymbol{d}_{\boldsymbol{e}}=\mathbf{2}$ sobre $\sigma$ - quatorze nós para $(12,13,25,26,27,28,38,39,40,41,53,54$, 66 e 67). 
- Resultados para malha irregular 12x9 - enriquecimento com funções trigonométricas.

Para finalizar as análises do problema 2, serão apresentados alguns dos resultados dos ensaios numéricos realizados com enriquecimento das bases aproximativas, envolvidas na FHMT, por funções trigonométricas (ver tabela 7.20). Como funções enriquecedoras foram adotadas $\operatorname{sen}\left\lfloor\left(y-y_{n \dot{b}}\right)^{2}\right\rfloor$ e $\cos \left\lfloor\left(x-x_{n \dot{b}}\right)^{2}\right\rfloor$ para enriquecimento dos campos de domínio e $\operatorname{sen}\left\lfloor\left(\boldsymbol{y}-\boldsymbol{y}_{\boldsymbol{n} \boldsymbol{o}}\right)^{2}\right\rfloor$ para o enriquecimento do campo de deslocamentos no contorno.

Da tabela 7.20, verifica-se que dadas as peculiaridades do problema que acaba por exigir uma malha mais refinada, não se conseguiu uma aproximação maior em relação ao nível de energia de deformação exato do problema 2 . .

Tabela 7.20 - Resultados gerais obtidos para o problema 2 com malha irregular $12 \mathrm{x} 9 \mathrm{e}$ o enriquecimento realizado com funções trigonométricas.

\begin{tabular}{|c|c|c|}
\hline \multirow{2}{*}{ Condições de Enriquecimento } & Energia de Deformação & \multirow{2}{*}{$\begin{array}{l}\text { Teste do } \\
\text { Mosaico }\end{array}$} \\
\hline & $U_{\text {exatoP2 }}=66,01 \quad U_{E M}=65,98$ & \\
\hline Sem enriquecimento & 62,05 & Ok! \\
\hline Todos os nós enriquecidos - $\sigma$ e $u-\Omega-\operatorname{sen}\left[\left(y-y_{n o}\right)^{2}\right]$ & 61,85 & Ok! \\
\hline Todos os nós enriquecidos $-\sigma-\Omega-\operatorname{sen}\left[\left(y-y_{n o}\right)^{2}\right]$ & 61,41 & Ok! \\
\hline Quatorze nós enriquecidos - $\sigma$ e $u-\Omega-\operatorname{sen}\left[\left(y-y_{n o}\right)^{2}\right]$ & 62,02 & Ok! \\
\hline Quatorze nós enriquecidos $\left.-\sigma-\Omega-\operatorname{sen}\left[\left(y-y_{n o ́}\right)^{2}\right]\right)$ & 61,75 & Ok! \\
\hline Quatorze nós enriquecidos - $\sigma$ e $u-\Omega-\cos \left[\left(x-x_{n o}\right)^{2}\right]$ & 62,14 & Ok! \\
\hline Quatorze nós enriquecidos $-\sigma-\Omega-\cos \left[\left(x-x_{n o}\right)^{2}\right]$ & 61,88 & Ok! \\
\hline $\begin{array}{c}\left.\text { Quatorze nós enriquecidos }-\sigma-\Omega-\operatorname{sen}\left[\left(y-y_{n o ́}\right)^{2}\right]\right) \text { e } 1 \text { nó } \\
-u-\Gamma-\operatorname{sen}\left[\left(y-y_{n o}\right)^{2}\right]\end{array}$ & 61,79 & Ok! \\
\hline
\end{tabular}

No tocante aos campos de deslocamentos, houve uma maior compatibilidade entre os deslocamentos de domínio e contorno quando se enriqueceu somente o campo de tensões. Em geral, os deslocamentos do nó de referência de contorno (130) (Г) estão próximos aos do exemplo modelo. Ver tabela 7.21. 
Tabela 7.21 - Deslocamentos do nó de referência da malha de cobertura de domínio e contorno para a malha irregular $12 \times 9$.

\begin{tabular}{|c|c|c|}
\hline \multirow{3}{*}{ Condições de Enriquecimento } & \multicolumn{2}{|c|}{ Deslocamentos do nó de referência } \\
\hline & \multicolumn{2}{|c|}{$\boldsymbol{u}_{x E M}=0,2125 \mathrm{e} \boldsymbol{u}_{y E M}=0,0667$} \\
\hline & Deslocamento do nó $130(\Gamma)$ & Deslocamento do nó $130(\Omega)$ \\
\hline Sem enriquecimento & $u_{x}=0,2648$ e $u_{y}=0,0612$ & $u_{x}=0,1039$ e $u_{y}=0,2229$ \\
\hline $\begin{array}{c}\text { Todos os nós enriquecidos }-\sigma \text { e } u-\Omega \\
-\operatorname{sen}\left[\left(y-y_{n o}\right)^{2}\right]\end{array}$ & $u_{x}=0,2335 \mathrm{e} u_{y}=0,0644$ & $u_{x}=-0,0204$ e $u_{y}=-0,0189$ \\
\hline $\begin{array}{c}\text { Todos os nós enriquecidos - } \sigma-\Omega- \\
\operatorname{sen}\left[\left(y-y_{n o}\right)^{2}\right]\end{array}$ & $u_{x}=0,2306 \mathrm{e} u_{y}=0,0636$ & $u_{x}=0,2290$ e $u_{y}=0,0635$ \\
\hline $\begin{array}{c}\text { Quatorze nós enriquecidos - } \sigma-\Omega- \\
\operatorname{sen}\left[\left(y-y_{n o}\right)^{2}\right]\end{array}$ & $u_{x}=0,2376 \mathrm{e} u_{y}=0,0602$ & $u_{x}=0,2256$ e $u_{y}=0,0596$ \\
\hline
\end{tabular}

Apresentam-se nas figuras 7.35 e 7.36 os campos de tensões para algumas das condições de enriquecimento abordadas na tabela 7.20. Com esse tipo de enriquecimento, também se conseguiu uma melhor definição da zona de concentração de tensões na ponta da fenda (cota de 20 unidades de comprimento no lado esquerdo da chapa).

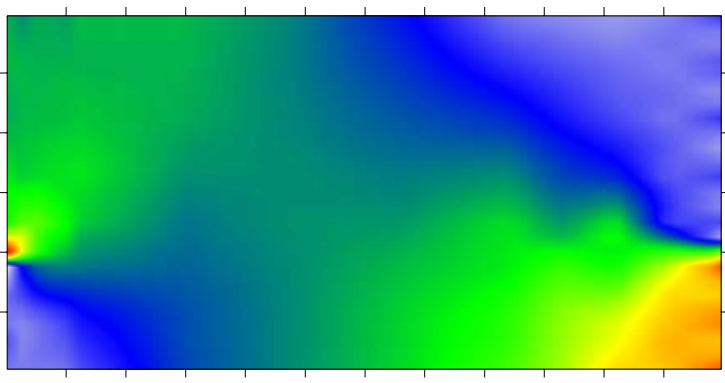

Sigma-X

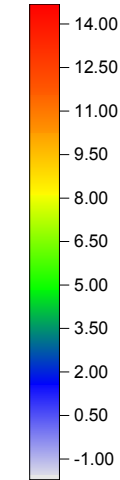

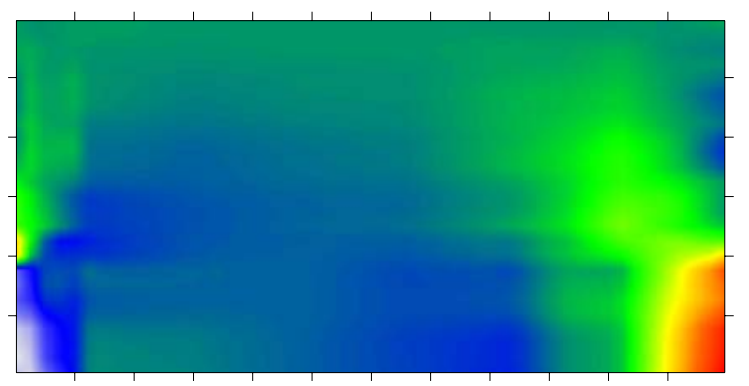

Sigma-y

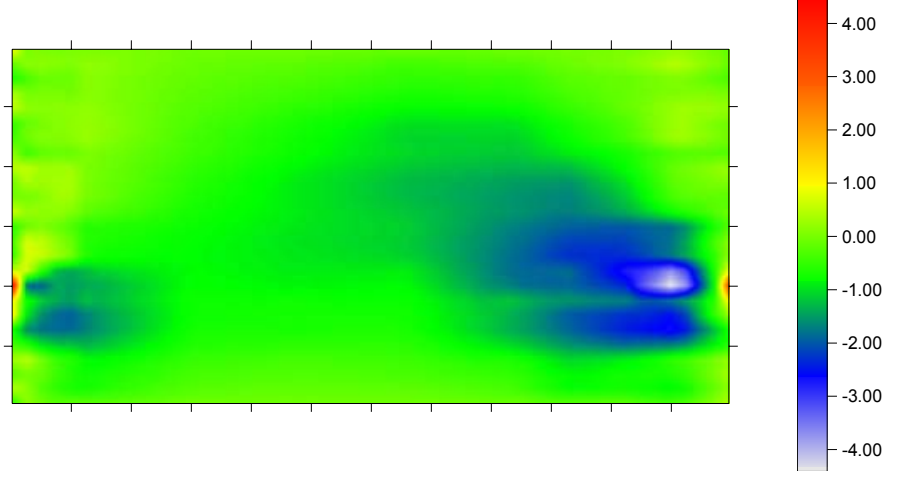

Tau-xy

Figura 7.35- Representação do campo de tensões com enriquecimento do tipo sen $\left\lfloor\left(y-y_{n \dot{o}}\right)^{2}\right\rfloor$ sobre $\sigma$ em $\Omega$ - todos os nós. 


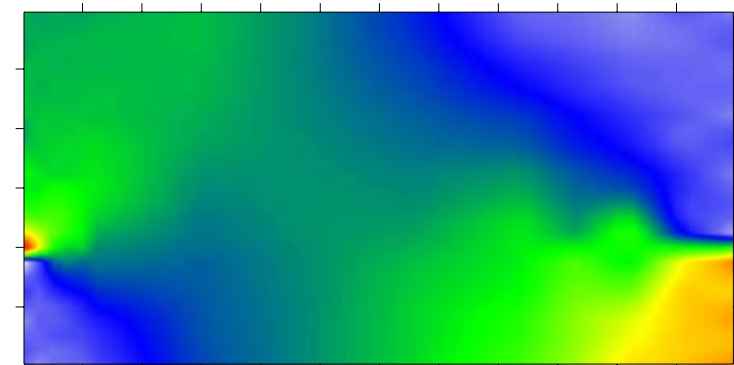

Sigma-x
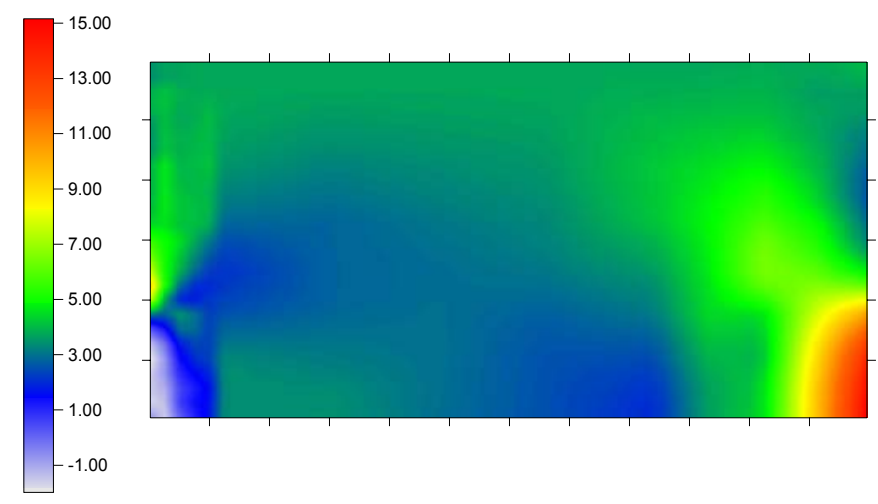

Sigma-y

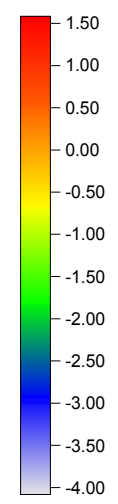

Tau-xy

Figura 7.36- Representação dos campos de tensão com enriquecimento do tipo $\operatorname{sen}\left\lfloor\left(\boldsymbol{y}-\boldsymbol{y}_{\boldsymbol{n} \dot{b}}\right)^{2}\right\rfloor$ sobre $\sigma$ e $u$ em $\Omega$ - todos os nós.

\subsection{Discussão dos Resultados.}

Inicialmente, deve-se observar que as considerações que seguem em relação à precisão dos resultados numéricos obtidos, têm por base o confronto entre os valores de referência e calculado para a energia de deformação. Porém uma outra norma poderia ser adotada para o confronto, como a norma de energia, obtendo-se, eventualmente, margens de erro menores. Portanto, a avaliação qualitativa depende da norma adotada e não esta sendo objeto de consideração rigorosa neste trabalho.

No estudo do problema 1 com as malhas regular $(8 \times 9)$ e irregular $(12 \times 15)$, utilizaram-se diferentes tipos de enriquecimento sobre as bases de aproximação dos campos de domínio e contorno envolvidos na FHMT. Nesse primeiro problema foram testados funções polinomiais, trigonométricas e polinômios especiais que proporcionam campos de tensões auto-equilibrados. Todos os ensaios numéricos englobando as várias possibilidades de enriquecimento, onde o Teste do Mosaico foi satisfeito, resultaram em energias de deformação próximas às energias dos exemplos modelos. 
Os dois graus de enriquecimento $\left(\boldsymbol{d}_{e}=\boldsymbol{1}\right.$ e $\left.\boldsymbol{d}_{e}=2\right)$ utilizados nos ensaios numéricos com polinômios especiais (funções de Airy) na malha regular $(8 \times 9)$, proporcionaram um nível da energia de deformação um pouco abaixo do nível de energia de deformação do exemplo modelo simulado no ANSYS ${ }^{\circledR}$. Já para a malha irregular (12x15), somente com o grau de enriquecimento $\boldsymbol{d}_{e}=\mathbf{2}$ o nível de energia de deformação ficou abaixo do valor de referência.

$\mathrm{O}$ enriquecimento das bases aproximativas com funções trigonométricas, no problema 1, só foi testada na malha irregular (12x15). Os casos de enriquecimento por $\operatorname{sen}\left\lfloor\left(y-y_{n \dot{b}}\right)^{2}\right\rfloor$ e $\cos \left[\left(x-x_{n \dot{b}}\right)^{2}\right\rfloor$, os níveis de energia de deformação foram bem próximos a do exemplo modelo. Vale salientar que, para esse tipo de enriquecimento, foram utilizados no mínimo cinco pontos de Gauss em cada direção do elemento de domínio e três no elemento de contorno para integração das matrizes com esse tipo de enriquecimento.

Os deslocamentos dos nós de referência das malhas de contorno e de domínio do problema 1, discretizado com a malha regular (8x9) sem enriquecimento, não foram compatíveis. Com o enriquecimento polinomial sobre as bases aproximativas do domínio, conseguiu-se recuperar a compatibilidade entre os deslocamentos nos mesmos nós de domínio e contorno. O enriquecimento polinomial sobre os campos de deslocamento no contorno, para a malha regular (8x9) não propiciou a compatibilidade entre os nós da malha de cobertura de domínio e contorno. Na malha irregular $(12 \times 15)$ em todas as situações onde o Teste do Mosaico foi satisfeito houve proximidade entre os deslocamentos da malha de cobertura de contorno e domínio do problema 1.

Em termos gerais, os deslocamentos do nó de referência da malha de cobertura de contorno do problema 1, para as malhas regular (8x9) e irregular (12x15) com todos os tipos de enriquecimento utilizados na análise desse problema, ficaram próximos aos deslocamentos dos exemplos modelos quando o Teste do Mosaico foi satisfeito e o nível da energia de deformação não foi muito distante da energia de deformação de referência.

No tocante aos campos de tensões do problema 1 com a malha regular $(8 \mathrm{x} 9)$, observou-se que as estimativas dos valores máximos e a própria representação dos três campos não foram próximas aos resultados obtidos com o exemplo modelo simulado no ANSYS $^{\circledR}$. O enriquecimento polinomial, na malha regular $(8 \times 9)$, proporcionou um aumento na estimativa dos valores máximos desses campos, mas não houve mudanças 
significativas na representação dos mesmos. Para algumas situações de enriquecimento com os polinômios auto-equilibrados já se conseguiu uma melhor representação dos campos com um aumento na estimativa dos seus valores máximos.

Para a malha irregular $(12 \times 15)$ as estimativas e representações dos campos de tensão já são muito próximas aos resultados do exemplo de referência do $\mathrm{ANSYS}^{\circledR}$. Alguns dos enriquecimentos polinomiais proporcionaram um aumento na estimativa dos valores máximos dos campos de tensão. Os enriquecimentos com polinômios autoequilibrados e funções trigonométricas na malha irregular (12x15), além de aumentar a estimativa dos campos de tensões, em algumas situações de enriquecimento, conseguiram capturar o efeito da concentração de tensão na região de descontinuidade do carregamento (nas cotas de 20 e 40 no lado vertical direito da chapa).

O problema 2 foi analisado com as malhas regular $(3 \times 3)$ e irregular $(12 \times 9)$. Além dos tipos de enriquecimentos aplicados no estudo do problema 1, utilizou-se no problema 2 , o enriquecimento com funções que se assemelham às soluções do campo de tensões e do campo de deslocamentos próximos à ponta de uma fenda fornecidos pela Mecânica da Fratura.

Como o problema 2 é de difícil simulação, justamente pela presença da fenda, requerendo uma malha mais refinada, não se conseguiu, em todos os ensaios numéricos um nível de energia de deformação próximo à energia de deformação exata do mesmo.

O estudo do problema 2 utilizando a malha regular $(3 \times 3)$ e as várias situações de enriquecimento com a função polinomial não resultaram em boas representações dos campos de deslocamento e de tensões . Já ao se enriquecer as aproximações dos campos de tensões com funções que se aproximam das soluções da fratura em poucos nós próximos à ponta da fenda, mesmo usando a malha regular $3 \times 3$, verificou-se que é possível representar de forma mais fiel a zona de concentração de tensão na região da ponta da fissura.

Analisando o problema 2 com a malha irregular (12x9) sem enriquecimento, já foi possível obter uma região de maior concentração de tensão entre as cotas 20 e 30 do lado vertical esquerdo da chapa, mesmo com a energia de deformação resultante da análise sem proximidade à energia de deformação exata do problema.

Quando na malha anterior foi realizado o enriquecimento polinomial sobre os campos aproximados de domínio, conseguiu-se uma melhor representação do efeito da concentração de tensão na cota 20 do lado vertical esquerdo (ponta da fissura). $\mathrm{O}$ enriquecimento com este tipo de função polinomial sobre as aproximações dos campos 
de tensão e deslocamento no domínio geraram níveis de tensão mais baixa, quando comparados com os níveis de tensão obtida com o enriquecimento exclusivo sobre a aproximação do campo de tensões no domínio. Por outro lado, o enriquecimento de ambos os campos aproximados no domínio proporcionou uma maior regularidade dos campos de tensão.

Para o problema 2, verificou-se que o enriquecimento sobre as aproximações no domínio somente, em nós próximo à ponta da fissura não propiciam a compatibilidade entre os campos de deslocamentos no domínio e contorno. A compatibilidade entre esses dois campos é recuperada quando o enriquecimento sobre os campos do domínio é realizado ao mesmo tempo em nós próximos à ponta da fissura e em nós da região da aplicação do carregamento.

$\mathrm{Na}$ malha irregular (12x9) com o enriquecimento pela solução da fratura somente sobre a aproximação do campo de tensões no domínio, ou simultaneamente sobre as aproximações do campo de tensões e de deslocamento no domínio, a região de concentração de tensões na ponta da fenda (cota 20 no lado vertical esquerdo da chapa) ficou bem definida. As energias de deformação nos casos de enriquecimento citados ficaram próximas à energia de deformação para a situação sem enriquecimento.

Também foi possível recuperar o efeito da concentração de tensões na ponta da fenda quando se realizou o enriquecimento das bases aproximativas com os polinômios especiais e funções trigonométricas. Mesmo com um nível de energia de deformação um pouco abaixo da exata, obteve-se uma boa proximidade dos deslocamentos do nó de referência das malhas de cobertura de domínio e contorno em relação ao valor indicado pelo exemplo modelo.

Sobre a escolha do número de pontos de Gauss necessários para integração das matrizes que guardam as aproximações da FHMT com a possibilidade de enriquecimento nodal, observou-se que para as funções enriquecedoras do tipo polinomial, a lei representada para a obtenção do número de pontos de Gauss em função do maior grau do polinômio enriquecedor, mostrou-se eficiente.

No enriquecimento por funções trigonométricas ou aproximações das soluções da Mecânica da Fratura, o número de pontos de Gauss pode influenciar diretamente a qualidade da resposta. Com as funções trigonométricas existe um número mínimo de pontos de Gauss que deve ser utilizado para que o nível de energia de deformação não fique distante do valor da energia de deformação de referência. 
Já o enriquecimento com aproximações das soluções da Mecânica da Fratura exige uma grande quantidade de pontos de Gauss. Isso ficou evidente na tentativa de enriquecer todo o domínio do problema 2 com esse tipo de função enriquecedora.O nível de energia muito baixo obtido indicou que a quantidade de pontos de Gauss adotado não foi suficiente para integração da função presente em todo o domínio.

Em relação ao Teste do Mosaico verificou-se que o mesmo está ligado a solvibilidade do sistema de equações lineares discretas que resulta da FHMT com enriquecimento nodal e dado pela eq.(3.32). Entretanto, este teste é somente uma condição necessária para a solvibilidade da eq.(3.32). De fato para algumas situações de enriquecimento, mesmo com o Teste do Mosaico satisfeito, não se conseguiu convergência do método de Babuška (utilizado para resolução da eq.(3.32)) dentro da tolerância desejada.

Dessa forma, procurou-se estudar a condição suficiente para solvibilidade do sistema dado pela eq.(3.32), pesquisando-se os autovalores da matriz dos coeficientes da eq.(3.32) de um elemento quadrilateral com que um dos lados pertencente ao contorno do problema.

Sobre esse elemento, não vinculado, calculam-se os autovalores da matriz do sistema para todas as situações de enriquecimento que condicionaram a não convergência do método de Babuška. Nessas situações, verificou-se a presença de mais de três autovalores nulos (correspondentes aos movimentos de corpo rígido). Assim, devido ao número superior a três autovalores nulos seria impossível resolver o sistema de equações lineares dado por esse elemento. 


\section{Estudo da Condição de Babuška-Brezzi (inf-sup) Aplicada à FHMT}

\subsection{Introdução}

No capítulo 6, pontuou-se sobre o Teste do Mosaico para a FHMT. Esse estudo, baseado no trabalho de Zienkiewicz et al. (1986), está ligado a solvibilidade do sistema de equações discretas do problema e como já salientado é somente uma condição necessária, mas não suficiente para existência, unicidade e convergência da solução.

Nesta parte do trabalho apresenta-se a condição de Babuška-Brezzi (ou condição inf-sup), Babuška (1971) e Brezzi (1974), que é necessária e suficiente para a existência, unicidade e estabilidade de qualquer aproximação numérica obtida com o MEF. Inicialmente faz-se uma revisão da formulação variacional convencional em deslocamentos do problema elástico, para que se possa identificar e compreender todas as medidas envolvidas na condição de Babuška-Brezzi . Por outro lado, a verificação analítica dessa condição para um elemento finito associado a FHMT pode ser de difícil obtenção e, por essa razão, propõe-se uma alternativa numérica, baseada em Babuška (1996) e no trabalho de Chapelle e Bathe (1993), para verificar se os elementos quadrilaterais de quatro nós da FHMT satisfazem ou não essa condição.

\subsection{Problema Elástico Linear em Deslocamentos - Método dos Deslocamentos do MEF}

Como já pontuado, o problema elástico linear pode ser escrito em várias formulações variacionais ou fracas. Essencialmente todas elas têm a seguinte estrutura formal:

- $\quad$ Dois espaços de Hilbert $\boldsymbol{H}_{1}$ e $\boldsymbol{H}_{2}$; 
- Uma forma bilinear $\boldsymbol{B}(. .$.$) definida em \boldsymbol{H}_{1} \times \boldsymbol{H}_{2}$;

- Um funcional linear $\boldsymbol{F}($.$) definido em \boldsymbol{H}_{2}$.

Considere-se então o problema elástico linear escrito numa forma variacional decorrente da aplicação do Princípio dos Trabalhos Virtuais - PTV.

$$
\int_{\Omega} b \delta u d \Omega+\int_{\Gamma} \bar{t} \delta u d \Gamma=\int_{\Omega} \sigma \delta \varepsilon d \Omega
$$

onde $\boldsymbol{b}, \boldsymbol{u}, \overline{\boldsymbol{t}}, \sigma$ e $\varepsilon$ são respectivamente, numa representação matricial, vetores que guardam as componentes de forças volúmicas, de deslocamentos, de forças por unidade de superfície, do tensor de tensões e do tensor de deformações.

Nas integrais da eq.(8.4) $\delta \boldsymbol{u}$ é um vetor de deslocamentos virtuais e $\delta \varepsilon$ um vetor de deformações virtuais compatíveis com $\delta \boldsymbol{u}$. Considerando-se $\boldsymbol{b}$ e $\overline{\boldsymbol{t}}$ vetores conhecidos, para expressar a eq.(8.4) em função somente do campo de deslocamentos, utiliza-se da lei constitutiva e da relação de compatibilidade dadas, respectivamente, pelas eq.(2.5) e eq.(2.8). Assim, tem-se:

$$
\int_{\Omega} \delta u^{T}\left(L D L^{T}\right) u d \Omega=\int_{\Omega} \delta u^{T} b d \Omega+\int_{\Gamma_{t}} \delta u^{T} \bar{t} d \Gamma
$$

onde o operador $\boldsymbol{L}$ representa o operador divergente ou das derivadas parciais espaciais.

Finalmente, o problema (PVC) expresso pela eq.(8.5) pode ser representada da seguinte forma:

Encontre $\boldsymbol{u}_{0} \in \boldsymbol{H}_{1}$ tal que :

$$
\boldsymbol{B}\left(\boldsymbol{u}_{0}, v\right)=\boldsymbol{F}(\boldsymbol{v}), \quad \forall v \in \boldsymbol{H}_{2}
$$

Na eq.(8.6), tem-se que:

- $\quad v$ representa o vetor dos deslocamentos virtuais;

- A forma bilinear é dada por $B(u, v)=\int_{\Omega} v^{T}\left(\boldsymbol{L} \boldsymbol{D} \boldsymbol{L}^{T}\right) u d \Omega$;

- O funcional linear $F(v)=\int_{\Omega} v^{T} b d \Omega+\int_{\Gamma_{t}} v^{T} \bar{t} d \Gamma$ 
$\mathrm{Na}$ hipótese de restrições homogêneas sobre os deslocamentos na parte vinculada do contorno, admite-se ainda que $\boldsymbol{H}_{1}=\boldsymbol{H}_{2}$, e por definição,

$$
H_{1}=H_{2}=\left\{v \mid v \in P^{2}(\Omega) ; \frac{\partial v_{i}}{\partial x_{j}} \in P^{2}(\Omega), i, j=1,2,3 ; v_{\left.i\right|_{T}}=0, i=1,2,3\right\}
$$

Na relação anterior $\boldsymbol{P}^{2}(\Omega)$ é o espaço de Sobolev, definido como o espaço das funções quadrado-integráveis no domínio $\Omega$ considerado. Formalmente:

$$
P^{2}(\Omega)=\left\{w \in \Omega \mid \int_{\Omega}\left(\sum_{i=1}^{3}\left(w_{i}\right)^{2}\right) d \Omega=\|w\|_{P^{2}(\Omega)}^{2}<+\infty\right\}
$$

Nota-se que, tendo-se em vista a definição anterior, qualquer elemento $\boldsymbol{v}$ em $\boldsymbol{H}_{2}$ deve satisfazer com a nulidade as condições de contorno essenciais do problema e ainda

$$
\int_{\Omega}(v)^{2} d \Omega<\infty \text { e } \int_{\Omega}\left(\frac{\partial v_{i}}{\partial x_{j}}\right)^{2} d \Omega<\infty, i=1,2,3 \text { e } j=1,2,3
$$

o que tem a ver com a existência de solução para o problema em questão.

Vale salientar que, nessas condições, usualmente $\boldsymbol{H}_{\mathbf{1}}$ e $\boldsymbol{H}_{\mathbf{2}}$ representam, respectivamente, espaços de deslocamentos ou deslocamentos virtuais de energia finita. Para maiores detalhes sobre os espaços de Hilbert e Sobolev ver: Bathe (1996), Schwab (1998) e Brezzi e Fortin (1991).

A forma variacional descrita é usada para a determinação de uma solução aproximada do PVC. Nesse sentido, definem-se:

Espaços lineares $n$-dimensionais

$$
S_{1}^{n} \subset H_{1}, S_{2}^{n} \subset H_{2}
$$

e uma solução aproximada $\boldsymbol{u}_{\boldsymbol{n}}$ que se caracteriza por verificar a seguinte relação:

$$
B\left(u_{n}, v_{n}\right)=F\left(v_{n}\right), \forall v_{n} \subset S_{2}^{n}
$$

Deseja-se também que a solução aproximada verifique a seguinte condição:

$$
\left\|u_{n}-u_{0}\right\|_{H_{1}} \rightarrow 0 \text { com } n \rightarrow \infty
$$


onde $\boldsymbol{u}_{\mathbf{0}}$, conforme a eq.(8.6), representa a solução exata.

\subsubsection{Problema Bem Colocado}

Para garantir que o problema dado pela eq.(8.6) tenha boas propriedades, ou que seja bem colocado, ele deve apresentar as seguintes condições:

\section{1) Continuidade}

Um requerimento mínimo sobre $\boldsymbol{B}(\boldsymbol{u}, \boldsymbol{v})$ e $\boldsymbol{F ( v )}$ para que a eq.(8.6) tenha sentido é a continuidade, baseada na idéia que pequenas perturbações nos argumentos produzam pequenas variações naquelas formas. De modo mais formal, devem existir números $\boldsymbol{C}_{\boldsymbol{B}}>\mathbf{0}$ e $\boldsymbol{C}_{\boldsymbol{F}}>\mathbf{0}$ tais que para todo $\boldsymbol{u} \in \boldsymbol{H}_{1}$ e $\boldsymbol{v} \in \boldsymbol{H}_{2}$

$$
|B(u, v)|<C_{B}\|\boldsymbol{u}\|_{H_{1}}\|v\|_{H_{2}},|F(v)| \leq C_{F}\|v\|_{H_{2}}
$$

onde $\|\cdot\|_{H_{1}},\|\cdot\|_{H_{2}}$ representam normas em $\boldsymbol{H}_{1}$ e $\boldsymbol{H}_{2}$, respectivamente.

Se a eq.(8.13) é satisfeita, denomina-se $\boldsymbol{F ( v )}$ como um funcional linear contínuo em $\boldsymbol{H}_{2}$. O conjunto desses funcionais pertence ao espaço linear completo $\boldsymbol{H}_{2}{ }^{\prime}$ denominado de espaço dual de $\boldsymbol{H}_{2}$. A menor constante $\boldsymbol{C}_{\boldsymbol{F}}$ na eq.(8.13) é a norma de $\boldsymbol{F}(\boldsymbol{v})$, dada por:

$$
\|\boldsymbol{F}\|_{\boldsymbol{H}_{2}{ }^{\prime}}=\sup _{\boldsymbol{0} \neq \boldsymbol{v} \in \boldsymbol{H}_{2}} \frac{|\boldsymbol{F}(\boldsymbol{v})|}{\|\boldsymbol{v}\|_{\boldsymbol{H}_{2}}}
$$

onde sup significa o valor supremo.

2) A condição de Babuška-Brezzi (inf-sup)

Sejam $\boldsymbol{H}_{1}$ e $\boldsymbol{H}_{2}$ espaços de Hilbert com normas \|\|$_{H_{1}}$ e \|\|$_{H_{2}}$, respectivamente. Sejam ainda $\boldsymbol{B}(. .):. \boldsymbol{H}_{\mathbf{1}} \times \boldsymbol{H}_{\mathbf{2}} \rightarrow \mathfrak{R}$ uma forma bilinear e $\boldsymbol{C}_{\boldsymbol{B}}$ e $\lambda$ escalares positivos, tais que:

$$
|B(u, v)|<C_{B}\|u\|_{H_{1}}\|v\|_{H_{2}} \forall u \in H_{1} \text { e } v \in H_{2}
$$

Então, 
- $\quad \inf _{u \neq 0 \in H_{1}} \sup _{v \neq 0 \in H_{2}} \frac{B(u, v)}{\|u\|_{H_{1}}\|v\|_{H_{2}}} \geq \lambda>0$

- $\sup \underset{\boldsymbol{B} \in \boldsymbol{H}_{1}}{\boldsymbol{( \boldsymbol { u } , v )}}>\mathbf{0} \quad \forall v \neq \mathbf{0} \in \boldsymbol{H}_{2}$

As notações inf e sup significam respectivamente, o ínfimo e o supremo.

Essas condições são necessárias e suficientes para que exista uma única solução $\boldsymbol{u}_{0} \in \boldsymbol{H}_{1}$ que satisfaça:

$$
B\left(u_{0}, v\right)=F(v) \forall v \in H_{2}
$$

para todo funcional linear contínuo $F$ em $\boldsymbol{H}_{2}$.

Por outro lado, se as eq.(8.15) e eq.(8.16) são satisfeitas, uma estimativa para $\boldsymbol{u}_{\mathbf{0}}$ é dada por:

$$
\left\|u_{0}\right\|_{H_{1}} \leq \frac{\|F\|_{H_{2}^{\prime}}}{\lambda}=\frac{1}{\lambda} \sup _{v \neq 0 \in H_{2}} \frac{|F(v)|}{\|v\|_{H_{2}}}
$$

de modo que uma mudança suave em $\boldsymbol{F} \in \boldsymbol{H}_{2}{ }^{\prime}$ produz uma mudança também suave em $\boldsymbol{u}_{\mathbf{0}}$

O MEF foi formulado no item anterior, onde se definiram sub-espaços, dados respectivamente por $\boldsymbol{S}_{1}^{n} \subset \boldsymbol{H}_{1}$ e $\boldsymbol{S}_{2}^{n} \subset \boldsymbol{H}_{2}$. Se o problema original é bem colocado, o desempenho do método depende somente das escolhas de $\boldsymbol{S}_{1}^{n}$ e $\boldsymbol{S}_{2}^{n}$.

Assumindo-se então que $\boldsymbol{B}(\boldsymbol{u}, \boldsymbol{v})$ satisfaça as eq.(8.13), eq.(8.15) e eq.(8.16), a forma discreta da eq.(8.15) pode ser escrita como:

$$
\inf _{u \neq 0 \in S_{I}^{n}} \sup _{v \neq 0 \in S_{2}^{n}} \frac{B(u, v)}{\|u\|_{H_{1}}\|v\|_{H_{2}}} \geq \lambda(n)>0
$$

Note que a eq.(8.19) é uma analogia da eq.(8.15). Não é necessária uma analogia para a eq.(8.16), pois a mesma resulta atendida uma vez que $S_{1}^{n}$ e $S_{2}^{n}$ estão contidas em $\boldsymbol{H}_{1}$ e $\boldsymbol{H}_{2}$, respectivamente e abrangidas pela eq.(8.7).

Por outro lado, sendo $\boldsymbol{u}_{0} \in \boldsymbol{H}_{1}$ e $\boldsymbol{u}_{n} \in \boldsymbol{S}_{1}^{n}$ tais que: 


$$
\begin{aligned}
& B\left(u_{0}, v\right)=F(v), \forall v \in H_{2} \\
& B\left(u_{n}, v_{n}\right)=F\left(v_{n}\right), \forall v_{n} \in S_{2}^{n}
\end{aligned}
$$

Subtraindo-se a eq.(8.20) de eq.(8.21) e utilizando-se a primeira parte da desigualdade dada pela eq.(8.13), pode-se escrever que:

$$
\left\|u_{0}-u_{n}\right\|_{H_{I}} \leq\left(1+\frac{C_{B}}{\lambda(n)}\right) \underset{\chi \in S_{I}^{n}}{\inf }\left\|u_{0}-\chi\right\|_{H_{I}}
$$

A relação anterior serve para uma interpretação melhor sobre o escalar $\lambda$, que aparece na condição inf-sup, conforme o desenvolvimento que segue.

Sejam $\left\{\varphi_{i}\right\}_{i=1}^{n}$ e $\left\{\psi_{i}\right\}_{i=1}^{n}$ bases para $S_{1}^{n}$ e $\boldsymbol{S}_{2}^{n}$, respectivamente. Assim, se $\boldsymbol{u}_{n} \in \boldsymbol{S}_{1}^{n}$, pode-se escrever

$$
\begin{aligned}
& \boldsymbol{u}_{n}=\sum_{i=1}^{n} U_{i} \varphi_{i} \\
& \boldsymbol{v}_{n}=\sum_{j=1}^{n} \psi_{j} \delta U_{j}
\end{aligned}
$$

onde $\boldsymbol{U}_{\boldsymbol{i}}$ e $\boldsymbol{\delta} \boldsymbol{U}_{\boldsymbol{j}}$ são os deslocamentos e os deslocamentos virtuais nodais, respectivamente. Assim, pode-se escrever a eq.(8.21) da seguinte forma:

$$
\sum_{i=1}^{n} U_{i} B\left(\varphi_{i}, \psi_{j}\right)=F\left(\psi_{j}\right), j=1, \ldots, n
$$

O sistema anterior pode ser expresso em notação matricial, introduzindo-se os vetores: $\mathbf{u}=\left\{\boldsymbol{U}_{1}, \ldots, \boldsymbol{U}_{n}\right\}^{T}$ e $\mathbf{f}=\left\{\boldsymbol{F}\left(\psi_{1}\right), \ldots, \boldsymbol{F}\left(\psi_{n}\right)\right\}^{T}$. Assim sendo, tem-se:

\section{$B \mathbf{u}=\mathbf{f}$}

onde $\boldsymbol{B}$ é uma matriz $n \times n$ com elementos $\boldsymbol{B}_{i j}=\boldsymbol{B}\left(\varphi_{i}, \psi_{j}\right)$.

A condição $\lambda(\boldsymbol{n})>\mathbf{0}$ é necessária e suficiente para indicar a existência e unicidade de uma solução para a eq.(8.26). Observa-se que esta condição não diz nada sobre a relação entre $\boldsymbol{u}_{0}$ e $\boldsymbol{u}_{n}$, ou seja, ela é necessária mas não é suficiente para convergência. 
Assumindo-se que $S_{1}^{n}$ seja tal que, para qualquer $\boldsymbol{u}_{0} \in \boldsymbol{H}_{1}$,

$$
\underset{\chi \in S_{1}^{n}}{\inf }\left\|u_{0}-\chi\right\|_{H_{1}} \equiv \Phi^{n}\left(u_{0}\right) \rightarrow 0 \operatorname{com} n \rightarrow \infty
$$

então, se $\lambda(\boldsymbol{n}) \geq \lambda_{\mathbf{0}}>\mathbf{0}$, a eq.(8.22) implica:

$$
\left\|u_{0}-u_{n}\right\|_{H_{1}} \leq\left(1+\frac{C_{B}}{\lambda(n)}\right) \Phi^{n}\left(u_{0}\right) \rightarrow 0 \text { com } n \rightarrow \infty
$$

(havendo convergência, $\lambda$ é necessariamente positivo).

Por outro lado, se $\lambda(\boldsymbol{n}) \rightarrow \boldsymbol{0}^{+}$com $\boldsymbol{n} \rightarrow \infty$, mesmo se a eq.(8.27) é satisfeita, pode existir $\boldsymbol{u}_{\mathbf{0}} \in \boldsymbol{H}_{\mathbf{1}}$ tal que:

$$
\left\|u_{0}-u_{n}\right\|_{H_{1}} \not \supset \operatorname{com} n \rightarrow \infty
$$

( $\lambda$ positivo não garante convergência).

Em termos práticos, numa primeira etapa a condição de Babuška-Brezzi para uma certa aproximação $(\lambda(\boldsymbol{n})>\boldsymbol{0})$ indica existência de solução. Numa segunda etapa a constatação de $\lambda(\boldsymbol{n}) \rightarrow \mathbf{0}$ para sucessivas aproximações mais refinadas indica que pode haver problemas de convergência.

\subsubsection{Determinação Numérica de $\lambda(n)$ para uma Formulação em Deslocamentos}

Como salientado anteriormente, a condição dada pela eq.(8.15) pode servir ao controle das soluções obtidas com o MEF e por esta razão é de interesse determinar um valor de $\lambda(\boldsymbol{n})$. A determinação analítica de $\lambda(\boldsymbol{n})$, dependendo da formulação do MEF estudada, pode ser difícil, seguindo daí a necessidade de sua avaliação numérica. O desenvolvimento apresentado a seguir é baseado em Babuška (1996).

Sejam, então as aproximações definidas em $\boldsymbol{u} \in \boldsymbol{S}_{1}^{n}$ e $\boldsymbol{v} \in \boldsymbol{S}_{2}^{n}$, escritas em função de graus de liberdade nodais e bases de funções do espaço de elementos finitos definidos no item anterior. Primeiramente, reconhece-se que existem matrizes simétricas positivas-definidas $A_{1}$ e $A_{2}$, tais que:

$$
\|\boldsymbol{u}\|_{H_{1}}^{2}=\mathrm{u}^{\mathrm{T}} A_{1} \mathrm{u} ; \quad\|v\|_{H_{2}}^{2}=\mathrm{v}^{\mathrm{T}} A_{2} \mathrm{v}
$$


onde u e v são vetores que reúnem graus de liberdade nodais

Da mesma maneira, pode-se escrever a forma bilinear $\boldsymbol{B}(\boldsymbol{u}, \boldsymbol{v})$ como:

$$
B(u, v)=v^{\mathrm{T}} B \mathrm{u}
$$

Seja a condição dada pela eq.(8.19), escrita da seguinte forma:

$$
\inf _{u \neq 0 \in S_{I}^{n}} \frac{R(u)}{\|u\|_{H_{1}}} \geq \lambda(n)>0
$$

onde $\boldsymbol{R}(\boldsymbol{u})=\sup _{\boldsymbol{v} \neq \boldsymbol{0} \in S_{2}^{n}} \frac{\boldsymbol{B}(\boldsymbol{u}, \boldsymbol{v})}{\|\boldsymbol{v}\|_{\boldsymbol{H}_{2}}}$. Assim, com as eq.(8.30) e eq.(8.31), pode-se expressar $\boldsymbol{R}(\boldsymbol{u})$ da seguinte maneira:

$$
R(u)=\sup _{v \neq 0 \in S_{2}^{n}} \frac{B(u, v)}{\|v\|_{H_{2}}}=\sup _{v \neq 0 \in S_{2}^{n}} \frac{\mathrm{v}^{T} B \mathrm{u}}{\left(\mathrm{v}^{T} A_{2} \mathrm{v}\right)^{\frac{1}{2}}}
$$

Por outro lado como $A_{2}$ é simétrica e positiva definida, existe uma matriz simétrica positiva definida $A_{2}^{\frac{1}{2}}$ tal que $A_{2}^{\frac{1}{2}} A_{2}^{\frac{1}{2}}=A_{2}$, Babuška (1996). Definindo-se:

$$
A_{2}^{\frac{1}{2}} \mathrm{v}=\mathrm{s} \text { e v }=A_{2}^{\frac{-1}{2}} \mathrm{~s}
$$

tem-se que :

$$
\mathbf{S}^{\mathbf{T}} \mathbf{S}=\|v\|_{\mathrm{H}_{2}}^{2}
$$

e

$$
R(u)=\sup _{s} \frac{s^{T} A_{2}^{\frac{-1}{2}} B \mathrm{u}}{\left(s^{T} s\right)^{\frac{1}{2}}}
$$

Selecionando-se $\mathbf{s}=A_{2}^{\frac{-1}{2}} B \mathrm{u}$ :

$$
R(u) \geq \frac{\mathrm{u}^{T} B^{T} A_{2}^{\frac{-1}{2}} A_{2}^{\frac{-1}{2}} B \mathrm{u}}{\left(\mathrm{u}^{T} B^{T} A_{2}^{\frac{-1}{2}} A_{2}^{\frac{-1}{2}} B \mathrm{u}\right)^{\frac{1}{2}}}=\left(\mathrm{u}^{T} B^{T} A_{2}^{-1} B \mathrm{u}\right)^{\frac{1}{2}}
$$


a desigualdade de Schwartz fornece:

$$
\frac{\left|\mathrm{s}^{T} A_{2}^{\frac{-1}{2}} B \mathrm{u}\right|}{\left(s^{T} s\right)^{\frac{1}{2}}} \leq\left(\mathrm{u}^{T} B^{T} A_{2}^{\frac{-1}{2}} B \mathrm{u}\right)^{\frac{1}{2}}
$$

Assim:

$$
R(u)=\left(\mathrm{u}^{T} B^{T} A_{2}^{-1} B \mathrm{u}\right)^{\frac{1}{2}}
$$

Das eq.(8.32) e eq.(8.39), tem-se:

$$
\lambda^{2}(n)=\inf _{u \in S_{1}^{n}} \frac{\mathrm{u}^{T} B^{T} A_{2}^{-1} B \mathrm{u}}{\mathrm{u}^{T} A_{1} \mathrm{u}}
$$

Na expressão à direita da eq.(9.40) reconhece-se o quociente de Rayleigh, Bathe (1996), e portanto $\lambda^{2}(n)$ é o menor autovalor de um problema de autovalor generalizado dado por:

$$
B^{T} A_{2}^{-1} B x=\mu A_{1} x
$$

onde $\boldsymbol{x}$ e $\boldsymbol{\mu}$ são respectivamente os autovetores e os autovalores da eq.(8.41).

\subsection{A Condição de Babuška-Brezzi (inf-sup) aplicada à FHMT}

\subsubsection{O Teste Numérico da Condição de Babuška-Brezzi (inf-sup) aplicado à FHMT.}

\subsubsection{Introdução}

No item anterior, apresentou-se o procedimento numérico para determinação do $\lambda(\boldsymbol{n})$. Neste item desenvolve-se o teste numérico da condição inf-sup aplicado aos elementos quadrilaterais da FHMT com enriquecimento, tendo por base o trabalho de Chapelle e Bathe (1993). Antes, porém, comentam-se algumas características do teste numérico apresentado no trabalho de Chapelle e Bathe (1993). 


\subsubsection{O inf-sup Teste Aplicado a Problemas Incompressíveis}

Em Chapelle e Bathe (1993) o 'inf-sup teste' é aplicado a problemas que envolvem a condição de incompressibilidade. Naquele trabalho, chama-se a atenção para dois fenômenos comuns quando numa formulação em elementos finitos estão envolvidas grandezas como pressão e deslocamentos: o fenômeno de travamento ('locking') e a presença de modos espúrios.

Adotado um certo elemento finito, o fenômeno de travamento resulta de um desvio sobre a propriedade de convergência e pode ser detectado pelo 'inf-sup teste' quando se obtém $\lambda(\boldsymbol{n}) \rightarrow \mathbf{0}$, para uma seqüência de malhas mais refinadas.

Já a presença de modos espúrios pode afetar somente a solvibilidade, não tendo nenhuma relação com o travamento. De fato, Bathe (1996) ressalta que existindo modos espúrios, deve-se ignorar todos eles para o cálculo de $\lambda(\boldsymbol{n})$.

Assim sendo, aqueles autores sugerem que um elemento seja testado, no tocante ao travamento, pelo cálculo de $\lambda(\boldsymbol{n})$ com a eq.(8.41), utilizando-se malhas com refinamentos sucessivos. Ao menos três refinamentos são recomendados para prever se $\lambda(\boldsymbol{n})$ será limitado inferiormente por uma constante positiva. Em Chapelle e Bathe (1993) seguindo a formulação apresentada, são testados vários elementos e os valores numéricos comparados com a prova analítica da condição, quando disponível, obtendose para todas as situações confrontadas, resultados que confirmam, assim, a validade do procedimento.

\subsubsection{O inf-sup Teste Aplicado a FHMT com Enriquecimento}

Sejam as equações que governam a FHMT escritas da seguinte forma:

$$
\begin{aligned}
& \int_{\Omega} \delta \sigma^{T} f \sigma d \Omega+\int_{\Omega} u^{T}(L \delta \sigma) d \Omega-\int_{\Gamma_{t}} u_{\Gamma}^{T}(N \delta \sigma) d \Gamma=\int_{\Gamma_{u}} \bar{u}^{T}(N \delta \sigma) d \Gamma \\
& \int_{\Omega} \delta u^{T}(L \sigma) d \Omega=-\int_{\Omega} \delta u^{T} b d \Omega \\
& \int_{\Gamma_{t}} \delta u^{T}(N \sigma) d \Gamma=\int_{\Gamma_{t}} \delta u^{t} \bar{t} d \Gamma
\end{aligned}
$$


Como apresentado em Schwab (1998) para a formulação híbrida-mista de tensão, pode-se definir para as eq.(8.42), eq.(8.43) e eq.(8.44) uma forma bilinear $\boldsymbol{B}(.,$. e uma linear $\boldsymbol{F}($.$) , como segue abaixo:$

$$
\begin{gathered}
B(U, V)=\int_{\Omega} \delta \sigma^{T} f \sigma d \Omega+\int_{\Omega} u^{T}(L \delta \sigma) d \Omega-\int_{\Gamma_{t}} u_{\Gamma}^{T}(N \delta \sigma) d \Gamma+\int_{\Omega} \delta u^{T}(L \sigma) d \Omega+\int_{\Gamma_{t}} \delta u^{T}(N \sigma) d \Gamma \\
F(V)=\int_{\Gamma_{u}} \bar{u}^{T}(N \delta \sigma) d \Gamma-\int_{\Omega} \delta u^{T} b d \Omega+\int_{\Gamma_{t}} \delta u^{T} \bar{t} d \Gamma
\end{gathered}
$$

onde os espaços $\boldsymbol{U}=\left(\sigma, \boldsymbol{u}, \boldsymbol{u}_{\Gamma}\right)$ e $\boldsymbol{V}=\left(\delta \sigma, \delta \boldsymbol{u}, \delta \boldsymbol{u}_{\Gamma}\right)$ são definidos em $\boldsymbol{X}$ x $\boldsymbol{Y}$ com:

$$
\begin{aligned}
& \boldsymbol{X}=\left\{\left(\sigma, u, u_{\Gamma}\right): \sigma \in \boldsymbol{H}_{1} ; \boldsymbol{u}, \boldsymbol{u}_{\Gamma} \in \boldsymbol{P}^{2}(\Omega)\right\} \\
& \boldsymbol{Y}=\left\{\left(\delta \sigma, \delta u, \delta u_{\Gamma}\right): \delta \sigma \in \boldsymbol{H}_{1} ; \delta u, \delta u_{\Gamma} \in \boldsymbol{P}^{2}(\Omega)\right\}
\end{aligned}
$$

Da eq.(8.45), define-se a seguinte norma:

$$
\|U\|_{X}^{2}=\left\|\left(\sigma, u, u_{\Gamma}\right)\right\|_{X}^{2}=\int_{\Omega} \sigma^{2} d \Omega+\int_{\Omega}(L \sigma)^{2} d \Omega+\int_{\Gamma_{t}}(N \sigma)^{2} d \Gamma+\int_{\Omega} u^{2} d \Omega+\int_{\Gamma_{t}} u_{\Gamma}^{2} d \Gamma
$$

Dessa forma, ficam caracterizados todos os elementos envolvidos na eq.(8.15). Agora, para a aplicação do inf-sup teste ao elemento finito quadrilateral de quatro nós da FHMT com enriquecimento, é preciso determinar todas as matrizes envolvidas na eq.(8.41). Para isso, consideram-se as mesmas bases aproximativas para os campos de tensão e deslocamento no domínio e deslocamento no contorno, apresentadas no capítulo 3.

Então, seja um elemento quadrilateral com o lado vertical esquerdo contido no contorno do problema a ser analisado e um outro elemento qualquer do domínio. Ver figura 8.1 .

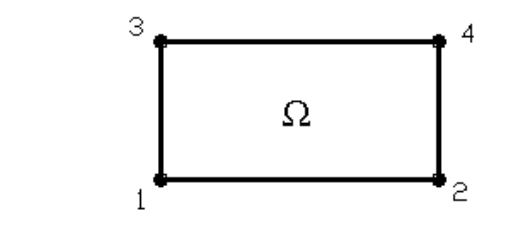

Elemento quadrllateral de domínlo

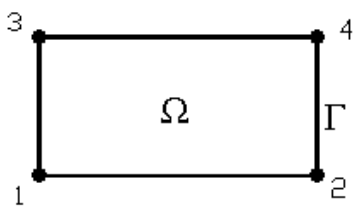

Elemento quadrllateral de domínlo com a lado vertlcal esquerdo no contorno

Figura 8.1 - Elementos quadrilaterais de quatro nós analisados pelo inf-sup teste. 
Das eq.(8.30), eq.(8.31) e eq.(8.49) determinam-se todas as matrizes da eq.(8.41). No apêndice (D) apresenta-se em detalhes a obtenção de cada uma dessas matrizes.

Igualmente à formulação estudada no trabalho de Chapelle e Bathe (1993), a FHMT também é suscetível a modos espúrios. No trabalho de Freitas, Almeida e Pereira (1999), salienta-se a presença dos modos espúrios estáticos, decorrentes do desenvolvimento de dependências lineares entre as três bases de aproximação, como um dos grandes entraves da FHMT. Nesse mesmo trabalho, destaca-se que este entrave pode ser superado se a estrutura do "solver" utilizado para solução do sistema de equações lineares que governa o modelo híbrido-misto for adaptada para identificar e descartar esses modos espúrios estáticos.

Quando são calculados os autovalores da matriz $\boldsymbol{B}$, referente a forma bilinear da eq.(8.26), (sem qualquer enriquecimento sobre as bases aproximativas da FHMT) observa-se claramente a presença de modos espúrios estáticos, pois além dos três autovalores nulos, que correspondem aos movimentos de corpo rígido, existem mais autovalores não nulos positivos, mas muito próximos de zero. Para efeito do inf-sup teste adota-se, aqui, o mesmo procedimento sugerido em Bathe (1996) e no trabalho de Freitas, Almeida e Pereira (1999), ou seja, descartam-se os autovalores próximos de zero.

Nos casos de enriquecimento exclusivo sobre os campos de deslocamentos da FHMT, tem-se claramente que o Teste do Mosaico não é satisfeito. Com essas condições de enriquecimento, verifica-se a presença de mais de três autovalores nulos (modos espúrios cinemáticos), que indica a impossibilidade de solvibilidade do problema. Dessa forma o inf-sup teste será aplicado somente ao elemento finito quadrilateral com as condições de enriquecimento onde o Teste do Mosaico é satisfeito, pois se o problema não tem solução não faz sentido estudar a existência e unicidade da resposta numérica.

Com os elementos ilustrados na figura 8.1 foram realizados os ensaios numéricos referentes ao inf-sup teste para várias condições de enriquecimento utilizando funções polinomiais (onde o Teste do Mosaico foi satisfeito) e com funções trigonométricas. Os resultados, plotados na forma $\log$ x $\log$, estão apresentados nas figuras que seguem, onde nas abscissas estão alocados o $\log \left(\frac{1}{N}\right)$, com $N$ o número de elementos na direção x (lado horizontal da chapa) e nas ordenadas o $\log (\lambda(\boldsymbol{n}))$. 


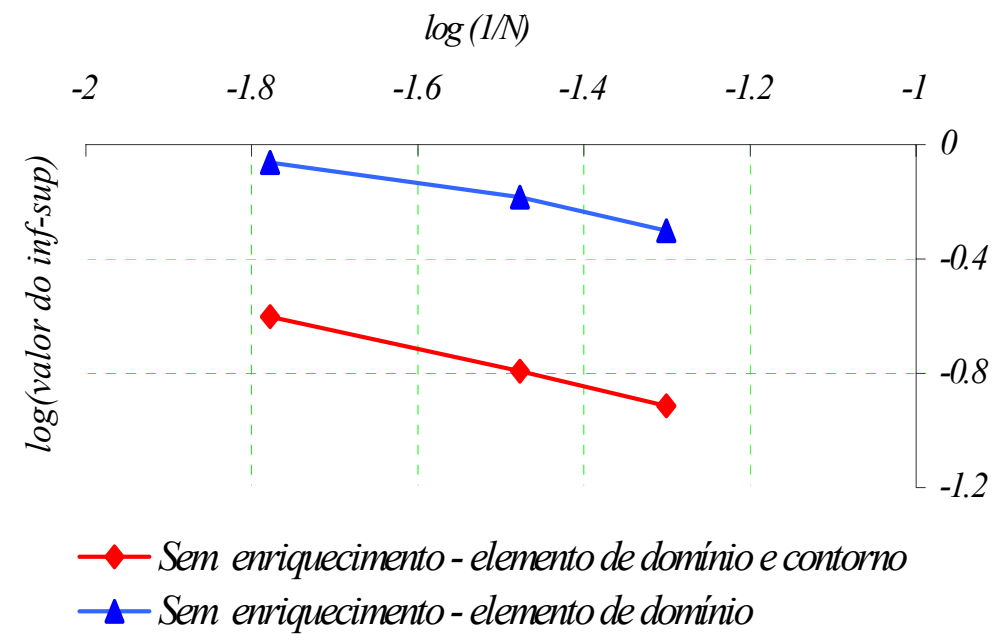

Figura 8.2 - inf-sup teste aplicado ao elemento quadrilateral de quatro nós sem enriquecimento.

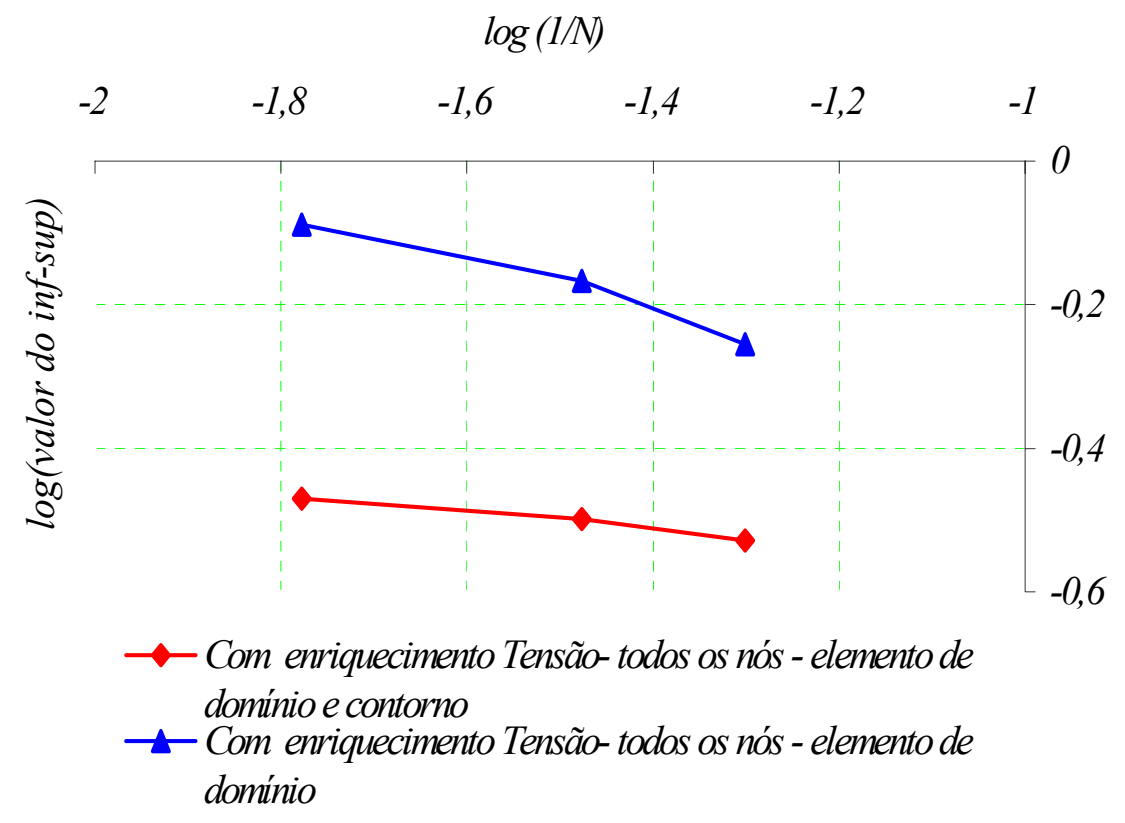

Figura 8.3 - inf-sup teste aplicado ao elemento quadrilateral de quatro nós com enriquecimento sobre o campo de tensões em todos os nós com a função $(y)^{2}$. 


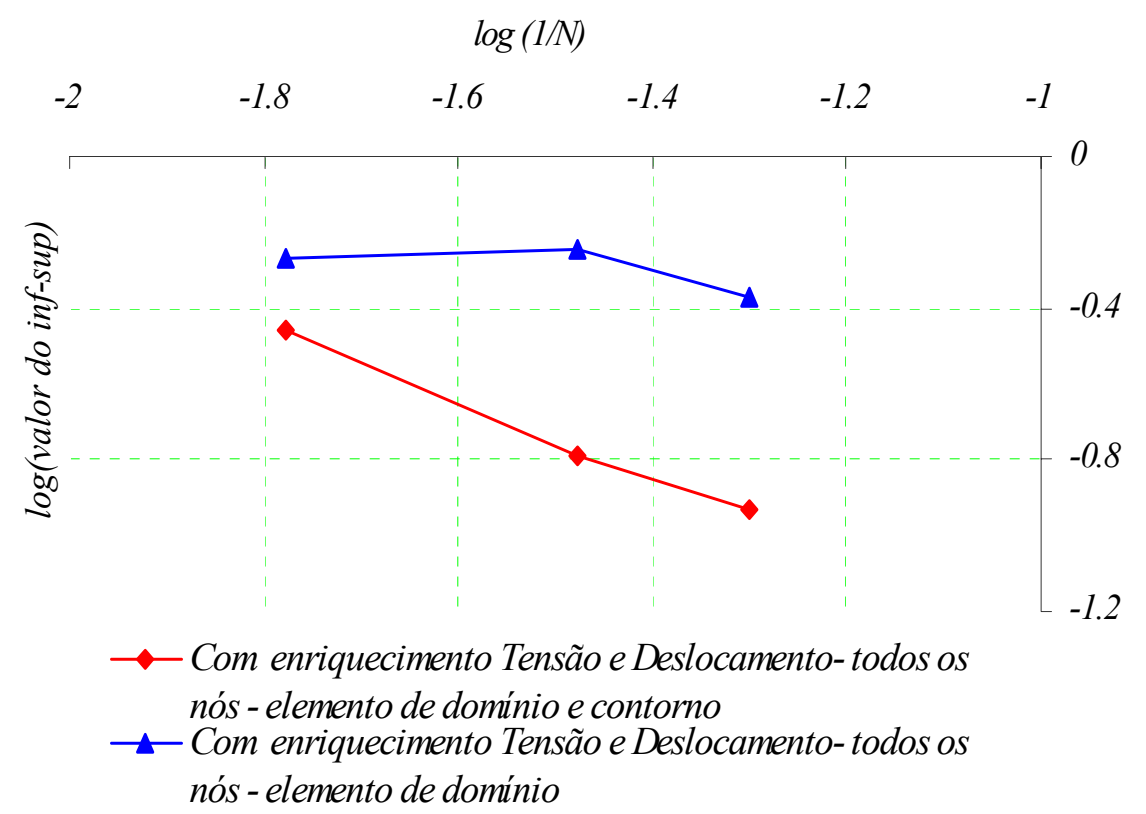

Figura 8.4 - inf-sup teste aplicado ao elemento quadrilateral de quatro nós com enriquecimento sobre o campo de tensões e deslocamentos no domínio em todos os nós com a função $(y)^{2}$.

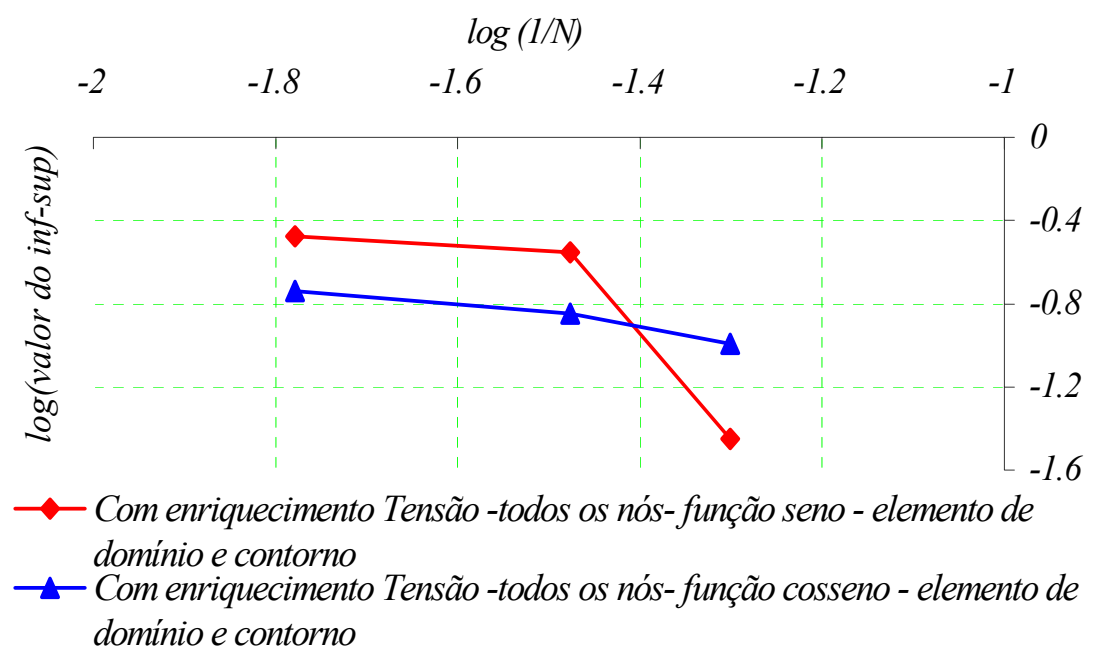

Figura 8.5 - inf-sup teste aplicado ao elemento quadrilateral de quatro nós com enriquecimento sobre o campo de tensões no domínio em todos os nós com as funções $\operatorname{sen}\left\lfloor(y)^{2}\right]$ e $\cos \left\lfloor(x)^{2}\right]$. 


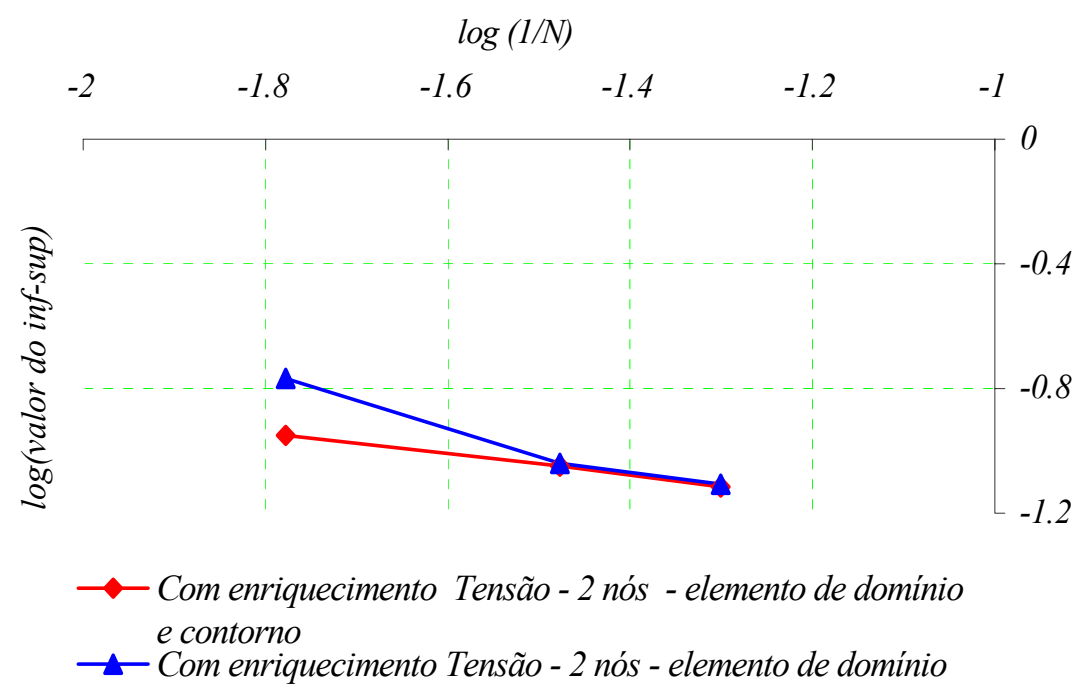

Figura 8.6 - inf-sup teste aplicado ao elemento quadrilateral de quatro nós com enriquecimento sobre o campo de tensões em dois nós com a função $(y)^{2}$.

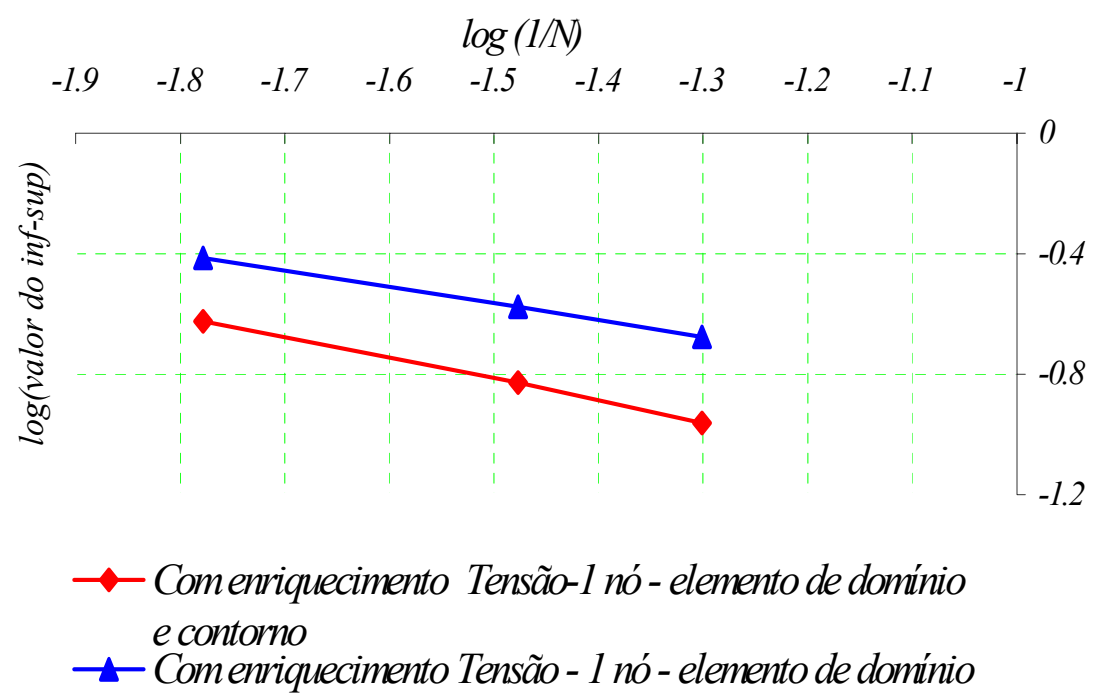

Figura 8.7 - inf-sup teste aplicado ao elemento quadrilateral de quatro nós com enriquecimento sobre o campo de tensões em um nó com a função $(y)^{2}$. 


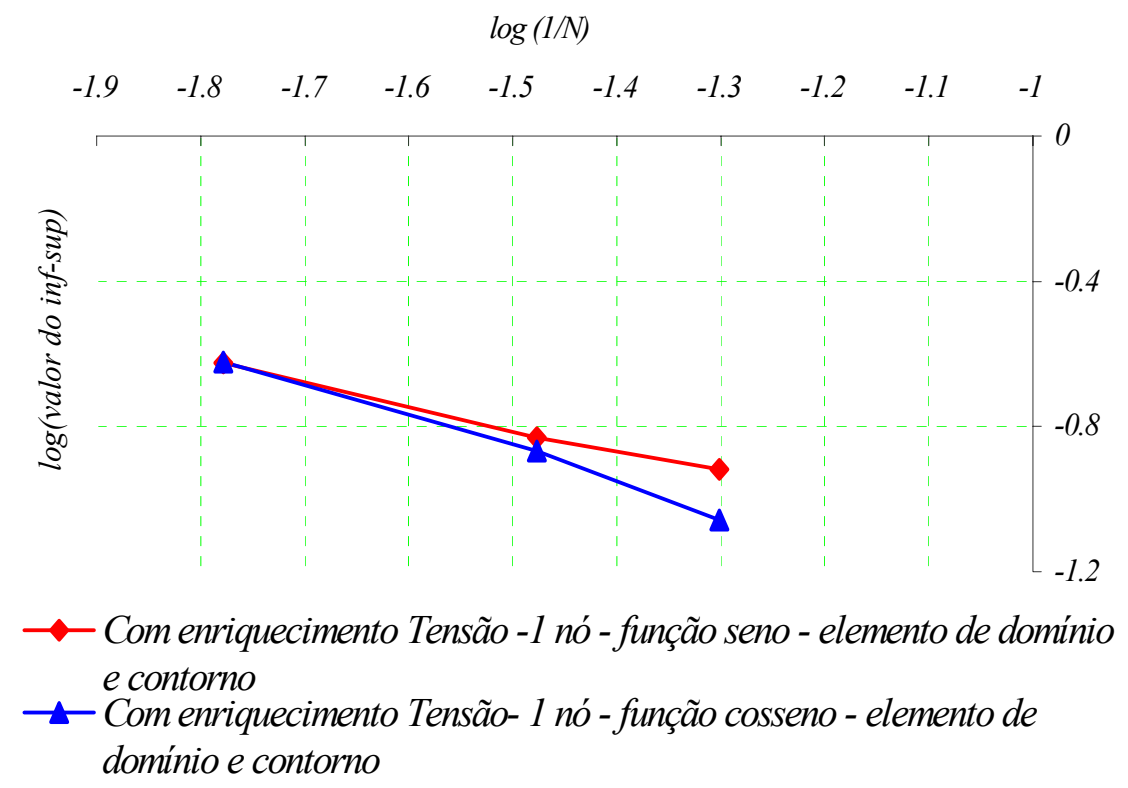

Figura 8.8 - inf-sup teste aplicado ao elemento quadrilateral de quatro nós com enriquecimento sobre o campo de tensões no domínio em um nó com as funções $\operatorname{sen}\left\lfloor(y)^{2}\right\rfloor$ e $\cos \left\lfloor(y)^{2}\right\rfloor$.

\subsection{Discussões sobre a Condição de Babuška-Brezzi (inf-sup) aplicado à FHMT com Enriquecimento Nodal}

Dentro do contexto da determinação das condições de convergência da resposta dada pela FHMT, iniciou-se o estudo da condição de Babuška-Brezzi que é necessária e suficiente para existência e estabilidade de qualquer resposta numérica para formulação em elementos finitos. Propôs-se um teste numérico (inf-sup teste), apresentado no item anterior, derivado da condição de Babuška-Brezzi para analisar se o Elemento Finito Híbrido-Misto de Tensão Quadrilateral com enriquecimento nodal pode proporcionar respostas estáveis ou não.

Na aplicação do inf-sup teste, verificou-se a tendência de obtenção de uma constante positiva $(\lambda(\boldsymbol{n})>\boldsymbol{0})$ para o elemento quadrilateral (domínio ou domínio/contorno) com todas as condições de enriquecimento polinomiais e trigonométricas propostas, como apresentam os gráficos das figuras 8.2, 8.3, 8.4, 8.5, 8.6 e 8.7, o que indica a verificação da condição de Babuška-Brezzi.

Em particular, quando se analisaram os resultados do inf-sup teste, pôde-se observar que com as condições de enriquecimento utilizando funções polinomiais e trigonométricas, nas quais o Teste do Mosaico foi satisfeito, não houve problemas de 
travamento da resposta, confirmando assim a validade das previsões do Teste do Mosaico sobre a solvibilidade do problema.

Comentou-se que nas situações com enriquecimento onde a condição necessária dada pelo Teste do Mosaico não foi satisfeita, não faria sentido aplicar o inf-sup teste. Mesmo assim, para esses casos, procurou-se calcular o valor do $\lambda(\boldsymbol{n})$ utilizando-se malhas com refinamentos sucessivos. Observou-se que após descartar os modos espúrios estáticos, nos três refinamentos analisados, obteve-se um menor autovalor $\lambda(\boldsymbol{n})=0$, confirmando, assim a robustez, do inf-sup teste proposto como ferramenta para a análise da existência e estabilidade da resposta numérica dada pela FHMT com enriquecimento nodal. 


\section{Conclusões e Considerações Finais}

Nesta pesquisa de mestrado estudou-se a formulação Híbrida-Mista de Tensão combinada com a possibilidade de aplicação do Método dos Elementos Finitos com enriquecimento nodal. $\mathrm{O}$ enriquecimento nodal, proposto originalmente no Método sem malha denominado Método das Nuvens "hp", foi aqui utilizado sobre os nós de uma malha de elementos finitos e consistiu na adição de novos graus de liberdade a um conjunto de nós pré-selecionados, aumentando o grau da aproximação sem a necessidade de refinamento da malha.

A discretização foi feita com elementos finitos quadrilaterais de quatro nós com funções de aproximação Lagrangianas bilineares para os campos de tensão e deslocamento no domínio e Lagrangianas lineares para o campo de deslocamento no contorno.

Para o enriquecimento nodal adotaram-se funções polinomiais, funções trigonométricas, polinômios correspondentes a distribuições de tensão auto-equilibradas ou mesmo funções especiais relacionadas às soluções dos problemas de fratura. Foram analisadas várias condições de enriquecimento sobre os campos de tensão e deslocamento no domínio e deslocamento no contorno.

No tocante à precisão dos resultados numéricos, utilizou-se o confronto entre os valores da energia de deformação dos exemplos modelos e os obtidos com a FHMT com enriquecimento nodal. Observa-se que poderiam ser utilizados outros tipos de norma (por exemplo, a norma de energia) para comparação de resultados e obter, eventualmente, margens de erro menores. Como já pontuado, a avaliação qualitativa depende da norma adotada e não é objeto de consideração rigorosa neste trabalho.

Os resultados numéricos obtidos apontaram, em geral, para uma avaliação positiva sobre a eficiência da alternativa numérica adotada. Particularmente, merecem destaque os resultados obtidos com enriquecimento pelo emprego de funções especiais, 
que permitiram identificar as zonas de concentração de tensões mesmo com malhas pouco refinadas. Entretanto, nem todas as combinações de enriquecimento conseguiram gerar níveis de energia de deformação satisfatória ou boa estimativa para os campos de tensão e deslocamento. Em alguns casos não se obteve sequer convergência. Por esta razão, e para fins de complementação do trabalho, passou-se a investigar as condições para convergência da resposta numérica da FHMT com estratégia de enriquecimento nodal da aproximação.

Inicialmente, com base no trabalho de Zienkiewicz et al. (1986), propôs-se um teste simples (denominado aqui de Teste do Mosaico) como indicativo da estabilidade do sistema de equações lineares resultante. Nesse teste, essencialmente, realiza-se uma comparação entre o número dos graus de liberdade das tensões e deslocamentos em “mosaicos" ou nuvens do domínio e contorno do problema.

Mostrou-se, por um lado, que o Teste do Mosaico está ligado à solvibilidade do sistema discreto. De fato, em todas as investigações onde o Teste do Mosaico não foi verificado, os resultados obtidos para os campos aproximados e para a energia de deformação não foram coerentes.

Por outro lado, observou-se que dependendo das funções e dos campos aproximados que são escolhidos para o enriquecimento, podem existir situações onde o Teste do Mosaico é satisfeito, mas sem convergência global da solução. Com essa última observação, conclui-se que o Teste do Mosaico é somente uma condição necessária para solvibilidade e estabilidade.

Para avaliar melhor a questão da solvibilidade do sistema de equações lineares discretas da FHMT com enriquecimento, passou-se a uma análise dos autovalores da matriz dos coeficientes do sistema. Para um único elemento finito quadrilateral foram determinados os autovalores, considerando-se as bases aproximativas dos três campos da FHMT e algumas das funções enriquecedoras utilizadas no trabalho. Conclui-se que não há convergência na resposta numérica quando para um determinado tipo de enriquecimento aparecem mais de três autovalores nulos (correspondentes aos movimentos de corpo rígido).

Este número adicional de autovalores nulos tem a ver, como será comentado mais adiante, com os modos cinemáticos espúrios.

É importante observar que o procedimento numérico utilizado para solução do sistema de equações lineares foi a combinação do método iterativo de Babuška juntamente com métodos para matrizes esparsas. Em todos os exemplos numéricos, 
onde as condições necessárias e suficientes para solvibilidade foram satisfeitas, esse procedimento mostrou-se bastante eficiente.

A suscetibilidade a modos espúrios estáticos e cinemáticos é um ponto de vulnerabilidade da FHMT com enriquecimento nodal. Os modos espúrios estáticos existem para a situação sem enriquecimento. Sabe-se que esses modos, quando identificados e descartados não interferem na solvibilidade do sistema de equações lineares (FREITAS, ALMEIDA e PEREIRA (1999)). O método de Babuška, pontuado anteriormente, consegue identificar os modos espúrios estáticos e eliminá-los. Já os modos espúrios cinemáticos podem surgir quando os campos de deslocamentos são exclusivamente enriquecidos ou simplesmente pela escolha inadequada da função enriquecedora utilizada para ampliar as bases aproximativas da FHMT (que foi o caso onde o Teste do Mosaico foi verificado, mas a solução não apresentou convergência). A presença desses modos leva a impossibilidade de existência e estabilidade da resposta para a FHMT com enriquecimento nodal da aproximação.

Para complementar o estudo da existência e estabilidade de solução, passou-se à análise da condição necessária e suficiente de Babuška-Brezzi (inf-sup) aplicada à FHMT com enriquecimento nodal. Esse tema constitui-se em outra contribuição original deste trabalho. Um teste numérico (inf-sup teste) dessa condição foi então proposto, tendo-se como referência os trabalhos de Chapelle e Bathe (1993), para verificar se os elementos finitos híbridos-mistos quadrilaterais com enriquecimento são estáveis ou não.

Os ensaios numéricos com o inf-sup teste revelaram que:

- O elemento finito híbrido-misto sem enriquecimento é estável, pois conseguiu-se $\lambda(\boldsymbol{n})>\boldsymbol{0}$, indicando verificação do critério de Babuška-Brezzi.

- O elemento finito híbrido-misto enriquecido por funções polinomiais e trigonométricas também é estável, pois obtiveram-se $\lambda(\boldsymbol{n})>\boldsymbol{0}$ nas várias situações testadas.

- O inf-sup teste é também condição necessária e suficiente para solvibilidade da eq.(3.32), pois em todas as situações onde o Teste do Mosaico não foi satisfeito ou mesmo sendo satisfeito, detectou-se a presença de modos espúrios cinemáticos, obteve-se $\lambda(\boldsymbol{n})=0$. 
Pode-se concluir, finalmente, que os ensaios numéricos realizados sobre a chapa tracionada simetricamente e a chapa com fenda e nos quais o inf-sup teste foi verificado, mostraram que a combinação dos elementos finitos híbridos-mistos de tensão com o enriquecimento nodal pode ser uma interessante alternativa para obtenção de uma boa precisão nas estimativas dos campos de tensão e deslocamento sem que seja necessário o emprego de malhas exageradamente refinadas. De fato, a utilização de discretizações simples e o enriquecimento sobre alguns nós enriquecidos em regiões de maior interesse, foram suficientes para gerar bons resultados, principalmente no tocante à representação dos campos de tensão.

Como propostas para desenvolvimentos futuros, citam-se:

- Implementação de novos elementos finitos para as análises via FHMT com enriquecimento da aproximação nodal.

- Avaliação da possibilidade de extensão da estratégia de enriquecimento nodal para as aproximações envolvidas na Formulação Híbrida Pura. Vale salientar que o enriquecimento proposto pelo Método das Nuvens 'hp' é realizado sobre bases aproximativas que constituem uma PU e por esta razão esta proposta deve ser cuidadosamente analisada, visto que as bases aproximativas de tensão da Formulação Híbrida Pura não constituem uma PU. 


\section{Referências Bibliográficas e Bibliografia Básica}

ANSYS RELEASE 5.5.1 (1998). Theory Manual. $3^{\text {rd }}$ edition. SAS IP, Inc.

AMARATUGA, K.; WILLIAMS, J.R. (1997). Wavelet-Galerkin solution of boundary value problems. Archives of Computational Methods in Engineering, v. 4, n. 3, p. 243 285.

BABUŠKA, I. (1971). Error bounds for finite element methods. Numerische Mathematik, v.16, p. 322-333.

BABUŠKA, I. (1973). The finite element method with lagrange multipliers. Numerische Mathematik, v.20, p. 179-192.

BABUŠKA, I. (1996). On the inf-sup (babuška-brezzi) condition. The University of Texas at Austin. Technical Report \#5. TICAM

BABUŠKA, I.; CALOZ, G.; OSBORN, J. E. (1994). Special finite element method for a classe of second order elliptic problems with rough coefficients. SIAM Journal on Numerical Analysis, v.31, n.4, p. 727-981.

BABUŠKA, I.; MELENK, J. M. (1997). The partition of unity method. International Journal for Numerical Methods in Engineering, v. 40, p. 727-758.

BABUŠKA, I. et al. (1996). Finite element method for solving problems with singular solutions. Journal of Computational and Applied Mathematics, v.74, p. 51-70.

BARROS, F. B. (2002). Métodos sem malha e método dos elementos finitos generalizados em análise não-linear de estruturas. Tese (Doutorado). Escola de Engenharia de São Carlos, Universidade de São Paulo.

BATHE, K. J. (1996). Finite element procedures. 2.ed. Prentice-Hall.

BELYTSCHKO, T.; LU, Y.; GU, L. (1994). Element-free Galerkin methods. International Journal for Numerical Methods in Engineering, v.37, p. 229-256.

BREZZI, F. (1974). On the existence, uniqueness and approximation of saddle point problems arising from lagrange multipliers. RAIRD, v.8 (r-2), p. 127-151. 
BUSSAMRA, F. L. S. (1999). Elementos finitos hibrido-trefftz: um modelo elastoplástico tridimensional. Tese (Doutorado). Escola Politécnica da Universidade de São Paulo.

CHAPELlE, D.; BATHE, K. J. (1993). The inf-sup test. Computers \& Structures, v. 47, n. $4 / 5$, p. $537-545$.

CUNHA, C. (2000). Métodos numéricos. 2.ed. Campinas: Editora da Unicamp.

DESAI, C. S.; ABEL, J.F. (1972). Introduction to the finite element: a numerical meyhod for engineering analysis. New York. Van Nostrand Reinhold Company.

DUARTE, C. A. (1995). A review of some meshless methods to solve partial differential equations. The University of Texas at Austin. Technical Report. TICAM.

DUARTE, C. A. (1996). The hp-cloud method. Tese (Doutorado) - The University of Texas at Austin.

DUARTE, C. A.; BABUŠKA, I.; ODEN, J. T. (2000). Generalized finite element methods for three-dimensional structural mechanics problems. Computers \& Structures, v. 77 , n. 2, p. 215-232.

DUARTE, C. A.; ODEN, J. T. (1995). Hp clouds - a meshless to solve boundary value problem. The University of Texas at Austin. Technical Report. TICAM.

DUARTE, C. A.; ODEN, J.T. (1996). Hp clouds - an hp meshless method. Numerical Methods for Partial Differential Equations. John Wiley \& Sons, p. 1 - 34.

FREITAS, J. A. T.; ALMEIDA, J. P. B. M.; PEREIRA, E. M. B. R. (1996). Nonconventional formulations for the finite element method. Structural Engineering and Mechanics, v.4, p. 655-678.

GOLUB, G. H.; LOAN, C. F. V. (1996). Matrix computation. 5.ed. Maryland. Johns Hopkins University Press.

HOFFMAN, K.; KUNZE, R. (1971). Algebra linear. São Paulo. Editora Polígono S. A.

IRONS, B.M.; AHMAD, S. (1980). Techniques of finite elements. Ellis Harwood. Chichester.

IRONS, B.M.; RAZZAQUE, A. (1972). Experience with the patch test for convergence of finite element method. Mathematical Foundations of Finite Element Method. Academic Press, p. 557-587.

LABORATORIO DE COMPUTAÇÃO CIENTÍFICA (1983). Curso de mecânica teórica e aplicada - módulo II - primeira parte. Rio de janeiro.

LANCASTER, P.; SALKAUSKAS, K. (1981). Surfaces generated by moving least squares methods. Mathematics of Computation, v. 37, n.155, p. 141-158. 
LANCASTER, P.; SALKAUSKAS, K. (1990). Curve and surface fitting. Academic press.

LISZKA, T.; ORKISZ, J. (1977). Finite difference methods of arbitrary irregular mesh in non-linear problems of applied mechanics. ${ }^{\text {th }}$ International Conference on Structural Mechanics in Reactor Technology, San Francisco, California.

LISZKA, T.; ORKISZ, J. (1980). The finite difference method at arbitrary irregular grids and its applications in applied mechanics. Computer \& Structure, v. 11, p. 83 -95.

LIU, W. K. et al. (1995). Reproducing kernel particles methods for structural dynamics. International Journal for Numerical Methods in Engineering, v. 8, p. 1655-1679.

MELENK, J. M. (1992). Finite element methods with harmonic shape functions for solving laplace's equation. Dissertação (Mestrado) - University of Maryland, College Park.

MELENK, J. M. (1995). On generalized finite element methods. Tese (Doutorado) University of Maryland, College Park.

MELENK, J. M.; BABUŠKA, I. (1996). The partition of unity finite element method: basic theory and applications. Computer Methods in Applied Mechanics and Engineering, v.139, p.289-314.

MONAGHAN, J.J. (1982). Why particle methods work. SIAM Journal on Scientific and Statistical Computing, v.3, p. 422-433.

MONAGHAN, J.J. (1994). Simulating free surface flows with sph. Journal of Computational Physics, v.110, p. 399-406.

NAYROLES, B.; TOUZOT, G.; VILLON, P. (1992). Generalizing the finite element method: diffuse approximation and diffuse elements. Computational Mechanics, v.10, p. 307-318.

OÑATE, E. (1995). Calculo de estructuras por el metodo de elementos finitos: análisis estático lineal. 2.ed. Barcelona: Artes Gráficas Torres S.A.

OÑATE, E.; IDELSOHN, S.; ZIENKIEWICZ, O. C. (1995). Finite point methods in computational mechanics. Report 67.

OÑATE, E. et al. (1996). A finite point method in computational mechanics applications to convective transport and fluid flow. International Journal for Numerical Methods in Engineering, v.39, p. 3839-3866.

OÑATE, E. et al. (1996). A stabilized finite point method for analysis of fluid mechanics problems. Computer Methods in Applied Mechanics and Engineering, v. 139, p. 315-346. 
ODEN, J. T.; DUARTE, C.A.; ZIENKIEWICZ, O. C. (1998). A new cloud - based hp finite element method. Computer Methods in Applied Mechanics and Engineering, v. 153 , p. $117-126$.

PAVLIN, V.; PERRONE, N. (1975). Finite diference energy techniques for arbitrary meshes. Computer \& Structures, v. 5, p. 45-58.

PIMENTA, P. M.; PROENÇA, S. P. B.; FREITAS, J. A. T. (2002). Elementos finitos híbridos mistos com enriquecimento nodal. J. M. Gaicolea, C. Mota Soares, M. Pastor y G. Bugeda (Eds.), Métodos Numéricos em Ingeniería V, SEMNI.

PIAN, T.H H.; TONG, P. (1969). Basis of finite element methods for solid continua. International Journal for Numerical Methods in Engineering, v. 1, n 1.

SCHWAB, CH. (1998). $p$ - and $h p$ - finite element methods: theory and applications in solid and fluid mechanics. Oxford University Press Inc.

SHERPAD, D. (1968). A two-dimensional functions for irregularly spaced data. ACM National Conference. p. 517-524.

STROUBOULIS, T.; BABUŠKA, I.; COPPS, K. (2000). The design and analysis of the generalized finite element method. Computer Methods in Applied Mechanics and Engineering, v. 181, n. 1-3, p. 43-69.

SZABÓ, B.; BABUŠKA, I. (1991). Finite element analysis. John Wiley \& Sons.

TIMOSHENKO, S. P.; GOODIER, J. N. (1980). Teoria da Elasticidade. Guanabara Dois S. A.

TORRES, I. F. R. (2003). Desenvolvimento e aplicação do método dos elementos finitos generalizados em análise tridimensional não-linear de sólidos. Tese (Doutorado). Escola de Engenharia de São Carlos, Universidade de São Paulo.

VALLIAPAN, S. (1981). Continuum Mechanics: Fundamentals. A. A. Balkema.

WASHIZU, K. (1975). Variational methods in elasticity and plasticity. Pergamon Press.

XU, Z. (1992). Applied Elasticity. John Wiley \& Sons.

ZIENKIEWICZ, O. C. (1980). El método de elementos finitos. Editorial Reverté S.A.

ZIENKIEWICZ, O. C. (2000). The finite element method-Volume 1: The Basis.5.ed. Butterworth Heinemann.

ZIENKIEWICZ, O. C.; LEFEBVRE, D. (1987). Three-field mixed approximetion and the plate bending problem. Communications in Applied Numerical Methods, v.3, p. 301309.

ZIENKIEWICZ, O. C. et al. (1986). The patch test for mixed formulation. International Journal for Numerical Methods in Engineering, v.23, p. 1873-1882. 


\section{APÊNDICE A - Solução do Sistema de Equações Gerado pela Aplicação do MEFG na FHMT - Método de Babuška}

O sistema de equações lineares discretas do modelo híbrido-mista, eq.(3.32), tem como características principais: a simetria e a alta esparsidade. Ao contrário dos sistemas de equações obtidos por meio da formulação clássica do MEF, a eq.(3.32), dependendo das bases utilizadas na aproximação e do tipo de enriquecimento realizado sobre os campos de tensão e deslocamento no domínio e deslocamento no contorno, é suscetível a modos espúrios cinemáticos e/ou estáticos. Então é possível que o sistema de equações representado pela eq.(3.32) seja indeterminado uma vez que não é possível a priori se eliminar todas as dependências lineares.

O método iterativo de Babuška é adotado neste trabalho para solução da eq.(3.32), pois com sua utilização, busca-se contornar a dependência linear entre linhas e colunas desse sistema de equações.

Para sua apresentação, considere-se a eq.(3.32) escrita da seguinte forma:

$$
\boldsymbol{A x}=\boldsymbol{b}
$$

onde

$$
A=\left[\begin{array}{ccc}
F & A_{\Omega} & -A_{\Gamma} \\
A_{\Omega}^{T} & 0 & 0 \\
-A_{\Gamma}^{T} & 0 & 0
\end{array}\right] ; x=\left[\begin{array}{c}
s_{\Omega} \\
q_{\Omega} \\
q_{\Gamma}
\end{array}\right] \text { e } b=\left[\begin{array}{c}
0 \\
0 \\
-Q_{\Gamma}
\end{array}\right]
$$

Com a ajuda da matriz diagonal dada por:

$$
\boldsymbol{B}=[\operatorname{diag}(F)]^{-\frac{1}{2}}
$$

O sistema representado pela eq.(A.1) pode ser balanceado conforme: 


$$
\bar{A} \bar{x}=\bar{b}
$$

onde

$$
\overline{\boldsymbol{A}}=\left[\begin{array}{ccc}
\boldsymbol{B} F \boldsymbol{B} & \boldsymbol{B} \boldsymbol{A}_{\Omega} & -\boldsymbol{B} \boldsymbol{A}_{\Gamma} \\
\boldsymbol{A}_{\Omega}^{T} \boldsymbol{B} & \boldsymbol{0} & \boldsymbol{0} \\
-\boldsymbol{A}_{\Gamma}^{T} \boldsymbol{B} & \boldsymbol{0} & \boldsymbol{0}
\end{array}\right] ; \overline{\boldsymbol{x}}=\left[\begin{array}{c}
\overline{\boldsymbol{s}}_{\Omega} \\
\boldsymbol{q}_{\Omega} \\
\boldsymbol{q}_{\Gamma}
\end{array}\right] \text { e } \overline{\boldsymbol{b}}=\left[\begin{array}{c}
\boldsymbol{0} \\
\boldsymbol{0} \\
-\boldsymbol{Q}_{\Gamma}
\end{array}\right]
$$

Nota-se que:

$$
\operatorname{diag}(B F B)=I
$$

$\mathrm{e}$

$$
\boldsymbol{s}_{\Omega}=\boldsymbol{B} \overline{\boldsymbol{s}}_{\Omega}
$$

A eq.(A.5) deve ser utilizada após se obter a solução da eq.(A.3). Seja então a seguinte aproximação de $\overline{\boldsymbol{A}}$ :

$$
\widetilde{\boldsymbol{A}}=\overline{\boldsymbol{A}}+\varepsilon \boldsymbol{I}
$$

onde $\varepsilon$ é uma número positivo pequeno. A eq.(A.6) garante que $\tilde{\boldsymbol{A}}$ seja positiva definida. O método de Babuška pode ser resumido no seguinte algoritmo a seguir:

1. $\boldsymbol{j}=\boldsymbol{0} ; \overline{\boldsymbol{x}}_{\boldsymbol{0}}=\mathbf{0} ; \bar{r}_{0}=\bar{b}$;

2. resolva $\tilde{A} a_{j}=\bar{r}_{j}$;

3. $\overline{\boldsymbol{x}}_{j+1}=\overline{\boldsymbol{x}}_{j}+\boldsymbol{a}_{j}$;

4. $\overline{\boldsymbol{r}}_{j+1}=\overline{\boldsymbol{r}}_{j}-\overline{\boldsymbol{A}}_{j} \boldsymbol{a}_{j}$;

5. $j \leftarrow j+1$

6. se $\sqrt{\frac{\overline{\boldsymbol{r}}_{j}^{T} \overline{\boldsymbol{r}}_{j}}{\boldsymbol{x}_{j}^{T} \overline{\boldsymbol{A}} \overline{\boldsymbol{x}}_{j}}}>\boldsymbol{t o l}$ volte para o passo 2.

onde $\boldsymbol{a}_{\boldsymbol{j}}$ é o erro na iteração $\boldsymbol{j}$ e $\overline{\boldsymbol{r}}_{\boldsymbol{j}}$ é o resíduo no iteração $\boldsymbol{j}$.

No passo 2 do algoritmo anterior o sistema deve ser resolvido por um método numérico que leve em conta a estrutura e a esparsidade da matriz $\widetilde{\boldsymbol{A}}$. Neste trabalho foram experimentados tanto os métodos diretos, como o de Choleski com armazenamento esparso, como iterativos como, o método dos Gradientes Conjugados pré-condicionado pela decomposição incompleta de Choleski de $\tilde{\boldsymbol{A}}$. 
A convergência de (A.7) tem-se mostrado rápida em todos os casos testados, onde os testes para análise da solvibilidade da eq.(3.32) são satisfeitos, com menos de três iterações para $\varepsilon=10^{-8}$ e $t o l=10^{-15}$, nas análises sem enriquecimento. Nas análises com enriquecimento mediante funções polinomiais adotaram-se os mesmos valores anteriores, enquanto que $\varepsilon=10^{-8}$ e $t o l=10^{-10}$ foram adotadas nas análises com enriquecimento usando funções não-polinomiais, trigonométricas e polinômios correspondentes a distribuições de tensão auto-equilibradas. 


\section{APÊNDICE B - Métodos sem Malha}

\section{B.1 Considerações Iniciais}

Nos métodos sem malha a solução aproximada do PVC é feita a partir de um conjunto de pontos nodais dispersos no domínio sem conectividade pré-estabelecida, não existindo assim, uma malha de elementos finitos.

Em Torres (2003), pontuam-se algumas das principais motivações limitações do MEF como para o desenvolvimento dos métodos sem malha. Entre as limitações destacam-se: a geração de malha, na formulação em deslocamentos do MEF, é realizada com grande custo computacional; o procedimento h-adaptativo numa certa região do problema demanda uma nova malha de elementos e, o MEF clássico não favorece a resolução de problemas no âmbito da mecânica da fratura.

Como serão evidenciadas mais adiante, as funções de forma da maioria dos métodos sem malha são obtidas com a utilização das funções aproximadoras do Método dos Mínimos Quadrados Móveis (MMQM) (LANCASTER; SALKAUSKAS, 1981,1990). No MMQM os elementos fundamentais para construção da aproximação local de um certo ponto do domínio são: uma base de funções (geralmente polinomiais), funções de ponderação e uma região de influência ou nuvem.

Em Barros (2002) foi apresentado um resumo das várias denominações dos métodos que eliminam ou reduzem em parte a necessidade de construção de uma malha de elementos finitos na discretização de um domínio. Reproduz-se este resumo a seguir:

- Hidrodinâmica de Partículas Regularizadas (Smoothed Particle Hydrodynamics-SPH), (MONAGHAN, 1982,1994);

- Método de Galerkin por Ondas Pequenas (Wavelet Galerkin Method), (AMARATUGA; WILLIAMS, 1997); 
- Método das Partículas com Reprodução de Núcleo (Reproducing Kernel Particle Method - RKPM), (LIU et al., 1995).

- Métodos baseados diretamente nas aproximações do Método dos Mínimos Quadrados Móveis (MMQM):

- Método dos Elementos Difusos, MED (Difuse Element MethodDEM), (NAYROLES; TOUZOT; VILLON, 1992);

- Método de Galerkin Livre de Elementos, MGLE (Element Free Galerkin Method - EFGM), (BELYTSCHKO; LU; GU, 1994);

- Método dos Pontos Finitos, MPF (Finite Point Method - FPM), (OÑATE; IDELSOHN; ZIENKIEWICZ, 1995), (OÑATE et al., 1996a, 1996b);

- Métodos baseados na definição da Partição da Unidade (PU):

- Método das Nuvens "hp" (hp-clouds Method), (DUARTE; ODEN, 1995), (DUARTE, 1996) e (DUARTE; ODEN, 1996 a, 1996b);

- Método dos Elementos Finitos da Partição da Unidade, MEFPU (Partition of Unity Finite Element Method - PUFEM), (MELENK, 1995), (MELENK; BABUŠKA, 1996) e (BABUŠKA, MELENK, 1997).

Mesmo não estando incluídos nesta listagem, alguns trabalhos em diferenças finitas são também considerados precursores no desenvolvimento dos métodos sem malha (Liszka e Orkisz $(1977,1980)$ e Pavlin e Perrone (1975)).

Os trabalhos dos dois últimos grupos, citados no resumo acima, se destacam pelo pioneirismo e consolidação dos métodos sem malha.

O Método das Nuvens "hp" apresenta como particularidade o processo de construção da sua família de funções aproximadoras. A denominação de "hp" do Método das Nuvens é devido a grande versatilidade do mesmo para combinar os 
enriquecimentos do tipo " $\mathrm{h}$ ", que introduz novos pontos nodais ao domínio, com os do tipo "p", que introduz funções de graus superior com novos parâmetros nodais a eles associados.

Mas a característica mais peculiar do método é que caso seja necessário o enriquecimento da aproximação, pode-se introduzir novos monômios ou outro tipo de função multiplicando a base de funções proposta inicialmente, sem a necessidade de novos pontos nodais na discretização original.

A única exigência para que se possa realizar o enriquecimento sobre as funções de forma originais do Método das Nuvens "hp", é que estas constituam uma Partição da Unidade (PU). Mas essa característica é intrínseca as funções de forma do Método das Nuvens "hp", pois estas são obtidas a partir das funções aproximadoras do MMQM que constituem uma PU.

\section{B.2 Formulação Geral dos Métodos sem Malha com Base no Método dos Mínimos Quadrados Móveis (MMQM)}

Para apresentação da formulação dos métodos sem malha, considere-se o conjunto de pontos nodais, distribuídos no domínio $\Omega$ e indicados por $\boldsymbol{Q}_{N}=\left\{\boldsymbol{x}_{1}, \boldsymbol{x}_{2}, \ldots, \boldsymbol{x}_{N}\right\}, \boldsymbol{x}_{j} \in \Omega$. Com centro em $\boldsymbol{x}_{j}$, defini-se a área de influência ou nuven $\omega_{j}$, com a região limitada por uma medida $\boldsymbol{r}_{j}$, representada por:

$$
\omega_{j}=\left\{x \in \Omega ;\left\|x-x_{j}\right\| \leq r_{j}\right\}
$$

$\mathrm{Na}$ eq.(B.1), $\boldsymbol{x} \in \Omega$ representa um ponto qualquer do domínio $\Omega$. A distribuição de pontos e nuvens é tal que a união de todas as nuvens constituí uma região $\mathfrak{I}_{N}$ que deve englobar o domínio $\Omega$ e seu contorno. Caracteriza-se essa região como:

$$
\mathfrak{I}_{N}=\bigcup_{j=1}^{N} \omega_{j}, \mathfrak{I}_{N} \supset \bar{\Omega}
$$

onde $\bar{\Omega}$ inclui o interior e o contorno da região $\Omega$.

Define-se também uma função ponderadora contínua $\boldsymbol{W}_{j}$, associada a cada nuvem $\omega_{j}$, que assume valores não-nulos somente nessa vizinhança do nó $\boldsymbol{x}_{\boldsymbol{j}}$. Estas funções que apresentam valor não-nulo apenas numa dada região do domínio são 
denominadas funções de suporte compacto. Formalmente, indica-se: $\boldsymbol{W}_{j} \in \boldsymbol{C}_{\mathbf{0}}^{q}\left(\omega_{j}\right)$, onde $\boldsymbol{q}$ representa grau de derivada contínua de $\boldsymbol{W}_{j}, \omega_{j}$ a vizinhança de $\boldsymbol{x}_{j}$ em que a função peso é definida e o índice $\boldsymbol{O}$ indica que a função peso $\boldsymbol{W}_{\boldsymbol{j}}$ tem valor diferente de zero somente no interior de $\omega_{j}$.

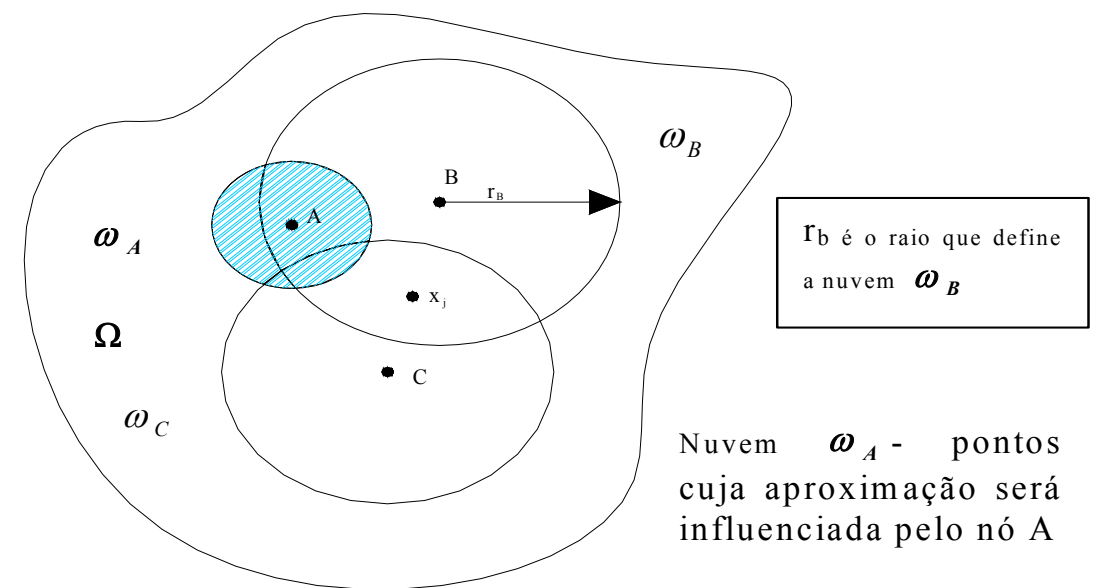

Ponto $\mathrm{x}_{\mathrm{j}}$ está na nuvem formada pela envoltória das nuvens $\mathrm{B}$ e $\mathrm{C}$.

Figura B.1 - Representação das nuvens e suas influências num domínio $\Omega \in \boldsymbol{R}^{2}$

Observa-se a na figura B.1 que um ponto $\boldsymbol{x}_{j}$ do domínio pode ser englobado por mais de uma nuvem. Dessa forma, a aproximação no ponto $\boldsymbol{x}_{\boldsymbol{j}}$ será determinada pela combinação das funções de forma locais ou nuvens em que ele esteja incluído.

Foi salientado que na grande maioria dos métodos sem malha, as funções aproximadoras são construídas pelo Método dos Mínimos Quadrados Móveis (MMQM). O MMQM é um método de geração de funções de aproximação introduzido por Shepard (1968). Com os trabalhos de Lancaster e Salkauskas (1981,1990), o MMQM foi aprimorado, mas permaneceu, na época, sem muita importância dentro das pesquisas em elementos finitos.

Para entendimento do MMQM e conseqüentemente da obtenção das funções de forma utilizadas pela maioria dos métodos sem malha, considere-se o problema simples de encontrar uma função de aproximação a partir de um conjunto de dados $\tilde{\boldsymbol{u}}_{j}, \boldsymbol{j}=1, \ldots, N$ associados a cada ponto $\boldsymbol{x}_{\boldsymbol{j}}$. Assume-se que a aproximação seja descrita pela seguinte expressão: 


$$
u(x) \approx \hat{u}(x)=p^{T}(x) \alpha(x)
$$

onde $p^{T}(x)=\left\{p_{1}(x), p_{2}(x), \ldots, p_{m}(x)\right\}$ é o vetor, de dimensão $m$, que guarda a base de funções adotada (geralmente polinomial) e $\alpha^{T}(x)=\left\{\alpha_{1}(x), \alpha_{2}(x), \ldots, \alpha_{m}(x)\right\}$ é o vetor, também de dimensão $\boldsymbol{m}$, que contêm as constantes da combinação linear que define a aproximação associada ao ponto $\boldsymbol{x}$.

Diferentemente do Método dos Mínimos Quadrados clássico, no MMQM, os parâmetros “ $\alpha$ ” são determinados pela minimização da função que reúne o quadrado das distâncias entre os $\boldsymbol{N}$ valores dados de $\tilde{\boldsymbol{u}}_{\boldsymbol{j}}$ (definido em cada ponto $\boldsymbol{x}_{\boldsymbol{j}}$ ) e os valores fornecidos pela aproximação $\hat{\boldsymbol{u}}\left(\boldsymbol{x}_{\boldsymbol{j}}\right)$ (nos mesmos pontos), ponderados por uma função $\boldsymbol{W}_{j}$ de suporte compacto $\left(\boldsymbol{C}_{\mathbf{0}}\right)$. Assim, a função a ser minimizada pode ser representada matematicamente pelo seguinte funcional quadrático $(\boldsymbol{J})$ :

$$
J(x)=\sum_{j=1}^{N} W_{j}\left(x_{j}-x\right)\left[\left(\tilde{u}_{j}-p^{T}(x) \alpha(x)\right)\right]^{2}
$$

A função ponderadora $\boldsymbol{W}_{j}$ é definida em cada ponto do domínio $\Omega$ e, nesse sentido, pode ser considerada como móvel, ou seja, acompanha o ponto onde se deseja definir a aproximação.

Para a minimização da eq.(B.4), impõe-se a condição necessária para estacionariedade de um funcional, ou seja, a nulidade de sua primeira derivada parcial em relação a cada coeficiente $\alpha_{i}, \boldsymbol{i}=\mathbf{1}, \ldots, \boldsymbol{m}$. Assim:

$$
\frac{\partial \boldsymbol{J}(\boldsymbol{x})}{\partial \alpha_{i}}=\frac{\partial \sum_{j=1}^{N} \boldsymbol{W}_{j}\left(\boldsymbol{x}_{j}-\boldsymbol{x}\right)\left[\tilde{u}_{j}-p^{T}(\boldsymbol{x}) \alpha(\boldsymbol{x})\right]^{2}}{\partial \alpha_{i}}=0
$$

A solução do sistema de $\boldsymbol{m}$ equações lineares geradas pela eq.(B.5), fornece a seguinte expressão para o cálculo do vetor $\alpha(x)$ :

$$
\alpha(x)=H^{-1}(x) \sum_{j=1}^{N} G_{j}(x) \tilde{u}_{j}
$$

onde a matriz $\boldsymbol{H}(\boldsymbol{x})$ é dada por: 


$$
H(x)=\sum_{j=1}^{N} W_{j}\left(x_{j}-x\right) p\left(x_{j}\right) p^{T}\left(x_{j}\right)
$$

e o vetor $\boldsymbol{G}_{j}(\boldsymbol{x})$ por:

$$
G_{j}(x)=W_{j}\left(x_{j}-x\right) p\left(x_{j}\right)
$$

Substituindo-se a eq.(B.6) na eq.(B.3), tem-se a nova apresentação para a aproximação da função $\boldsymbol{u}(\boldsymbol{x})$ :

$$
\hat{u}(x)=\sum_{j=1}^{N} \Psi_{j}(x) \tilde{\boldsymbol{u}}_{j}
$$

sendo as funções de forma $\Psi_{j}(\boldsymbol{x})$ dadas por:

$$
\Psi_{j}(x)=p^{T}(x) H^{-1}(x) G_{j}(x)
$$

Define-se assim, uma função de forma para cada ponto $\boldsymbol{x}_{\boldsymbol{j}}$.

A principal desvantagem do MMQM é que a sua aproximação é rapidamente destruída se o número de nós, $\boldsymbol{N}$, largamente excede o número $\boldsymbol{m}$ de termos do vetor $p(\boldsymbol{x})$ que guarda a base de funções adotadas. Isto faz sentido, pois qualquer aproximação por mínimos quadrados, o valor nodal aproximado $\hat{\boldsymbol{u}}\left(\boldsymbol{x}_{\boldsymbol{j}}\right)$ é diferente do valor $\tilde{\boldsymbol{u}}_{\boldsymbol{j}}$. Por outro lado, devemos ter como condição necessária (mas não suficiente) para que a matriz $\boldsymbol{H}(\boldsymbol{x})$ dada pela eq.(B.7) seja inversível é que este mesmo número de nós, $\boldsymbol{N}$, que contribuem para a aproximação seja maior ou igual ao número $\boldsymbol{m}$ de funções da base $p(x)$.

As funções de forma $\Psi_{j}(\boldsymbol{x})$ obtidas pelo MMQM, tem uma característica básica: elas constituem uma Partição da Unidade (PU). Direta ou indiretamente todos os métodos sem malha citados envolvem esse conceito. Convencionalmente, num ponto $\boldsymbol{x}$ o conjunto de funções de forma $\Psi_{j}(\boldsymbol{x})$, associados a subdomínios $\omega_{j}$, que o contém, constituem uma Partição da Unidade caso satisfaçam algumas condições. Estas condições estão apresentadas, por exemplo, em Torres (2003) e são transcritas abaixo: 
1) $\Psi_{j}(x) \in C_{0}^{\infty}\left(\omega_{j}\right)$ : as funções de forma pertencem ao conjunto de funções para os quais $\omega_{j}$ é um suporte compacto (incluindo-se suas derivadas até a ordem infinita). Isto significa que $\Psi_{j}(\boldsymbol{x})$ e suas derivadas assumem valores não-nulos apenas no interior da região $\omega_{j}$.

2) $\sum_{j=1}^{N} \Psi_{j}(x)=1$ : esta condição é essencial para assegurar que as funções de forma sejam capazes de reproduzir de modo exato uma função constante e assim garantir a convergência da aproximação à medida que novos pontos nodais sejam acrescentados ao domínio.

Para melhor compreensão da afirmação anterior, considere-se a aproximação dada pela eq.(B.9). Admitindo-se que a função a ser aproximada é uma constante, assim $\boldsymbol{u}(\boldsymbol{x})=\boldsymbol{k}$ e todos os valores nodais $\tilde{\boldsymbol{u}}_{\boldsymbol{j}}=\boldsymbol{k}, \forall \boldsymbol{j}=\mathbf{1 , 2}, \ldots, N$. Dessa forma a condição abordada neste item é necessária, pois:

$$
\begin{aligned}
& u(x) \approx \hat{u}(x)=\sum_{j=1}^{N} \Psi_{j} k=k \sum_{j=1}^{N} \Psi_{j}, \text { como } \sum_{j=1}^{N} \Psi_{j}=1, \text { então : } \\
& u(x) \approx \hat{u}(x)=k
\end{aligned}
$$

Com a verificação dessa segunda condição, tem-se que a aproximação $\widehat{\boldsymbol{u}}(\boldsymbol{x})$ representa exatamente qualquer constante. Isto implica, no caso da formulação clássica em deslocamentos do MEF, na capacidade de se reproduzir em movimentos de corpos rígidos, o que é um dos critérios utilizados pelo Teste do Mosaico ("Patch Test ") proposto por Irons e Razzaque (1972) e Irons e Ahmad (1980), como condição de convergência da solução discreta.

3) $\Psi_{j}(x) \geq 0$ em $\Omega$.

4) Todo sub-conjunto compacto de $\Omega$ intercepta apenas um número finito de suportes $\Psi_{j}(\boldsymbol{x})$.

As quatro condições acima são bastante restritivas e para fins práticos elas podem ser "relaxadas". Dessa forma, a primeira condição que exige suporte compacto até a ordem infinita nas derivadas, pode ser relaxada de tal forma que as funções de 
suporte compacto até uma ordem finita de derivada são ainda consideradas uma partição da unidade. Por isso, as funções Lagrangianas amplamente empregadas como funções de forma do MEF clássico, podem ser consideradas uma PU.

Destaca-se, mais uma vez, que as funções ponderadoras $\boldsymbol{W}_{\boldsymbol{j}}$ juntamente com a base polinomial $\boldsymbol{p}(\boldsymbol{x})$ adotada, são responsáveis pela continuidade da aproximação gerada a partir da função de forma $\Psi_{j}(\boldsymbol{x})$. Pode-se demonstrar que:

$$
\widehat{u}(x) \in C_{0}^{\min (k, l)}\left(\omega_{j}\right) \text { se }\left\{\begin{array}{l}
p(x) \in C^{k}\left(\omega_{j}\right) \\
W_{j}(x) \in C_{0}^{l}\left(\omega_{j}\right)
\end{array}\right.
$$

Por último, tem-se que qualquer função que seja uma combinação linear dos monômios da base $\boldsymbol{p}(\boldsymbol{x})$ pode ser exatamente reproduzida pela aproximação.

\section{B.3 O Método das Nuvens "hp"}

Em Duarte (1996), foi apresentada uma nova classe de funções de forma, denominada de família de Nuvens "hp". Essa nova família de funções é obtida basicamente pela multiplicação das funções de aproximação do MMQM, que constituem uma PU, por funções (geralmente polinomiais) linearmente independentes.

A família de Nuvens "hp" foi desenvolvida para gerar aproximações do tipo polinomial de ordem mais elevada a partir de uma base polinomial fraca sem a necessidade de inserir novos pontos nodais ao domínio discretizado. Com isso, eliminase um grande entrave encontrado quando se deseja aproximações, do tipo polinomial, de ordem mais elevada através do MMQM; essencialmente ligada à garantia de que a matriz $\boldsymbol{H}(\boldsymbol{x})$ seja inversível, ou seja, $\boldsymbol{N} \geq \boldsymbol{m}$.

Para apresentação formal da família de Nuvens "hp", considere-se, para o caso unidimensional, que as funções de forma $\Psi_{j}(\boldsymbol{x})$, eq.(B.10), tenham sido obtidas a partir de uma base polinomial $\boldsymbol{p}_{\boldsymbol{k}}=\left\{1, \boldsymbol{x}, \boldsymbol{x}^{\mathbf{2}}, \ldots, \boldsymbol{x}^{\boldsymbol{k}}\right\}$ e de funções ponderadoras $\boldsymbol{W}_{\boldsymbol{j}}$ $\in C_{0}^{q}\left(\omega_{j}\right)$, tal que $\boldsymbol{q} \geq \boldsymbol{k}$. Pode-se mostrar que qualquer polinômio de ordem menor ou igual a $\boldsymbol{k}$ pode ser exatamente reproduzido pela aproximação gerada. Para elevar a ordem da aproximação até a ordem $\boldsymbol{p}$, tal que $\boldsymbol{p}>\boldsymbol{k}$, passando então a corresponder a uma nova base $p_{p}=\left\{1, x, x^{2}, \ldots, x^{k}, \ldots, x^{p}\right\}$, define-se uma família de funções formada pela soma das funções de aproximação originais com as funções resultantes do produto 
de cada uma das funções originais pelas componentes do conjunto $\boldsymbol{p}_{p}-\boldsymbol{p}_{\boldsymbol{k}}=\left\{\boldsymbol{x}^{k+1}, \ldots, \boldsymbol{x}^{p}\right\}$. A classe de funções assim definida denomina-se família de funções de forma do Método das Nuvens "hp" e tem a seguinte representação:

$$
\mathfrak{I}_{N}^{k, p}=\left\{\left\{\Psi_{j}(x)\right\}_{j=1}^{N} \cup\left\{\Psi_{j}(x) x^{i}\right\}_{j=1}^{n}: i=k+1, \ldots, p\right\}
$$

Substituindo-se as funções apresentadas na eq.(B.12) na aproximação fornecida pela eq.(B.9), tem-se:

$$
\hat{u}(x)=\sum_{j=1}^{N} \Psi_{j}(x)\left[\tilde{u}_{j}+\sum_{i=k+1}^{p} x^{i} b_{j(i-k)}\right]
$$

onde $\boldsymbol{b}_{j(i-k)}$ são os parâmetros adicionais aos nós em função do enriquecimento analisado.

Segundo Duarte e Oden (1996), quando se utilizam as funções de forma da família do Métodos das Nuvens "hp", a taxa de convergência da resposta depende somente da base final $\boldsymbol{p}_{\boldsymbol{p}}$, ou seja, a base original $\boldsymbol{p}_{\boldsymbol{k}}$ não tem influência sobre ela. Por isso, é usual escolher como base inicial $\boldsymbol{p}_{\boldsymbol{k}}$ a base mais simples, $\boldsymbol{p}_{\boldsymbol{k}=\mathbf{0}}=\{\mathbf{1}\}$, eliminando-se assim, a necessidade de inversão da matriz $\boldsymbol{H}(\boldsymbol{x})$. Dentro dessas condições, as expressões da matriz $\boldsymbol{H}(\boldsymbol{x})$, do vetor $\boldsymbol{G}_{\boldsymbol{j}}(\boldsymbol{x})$ e das funções das funções de forma $\Psi_{j}(x)$ (denominadas, nessa situação, de funções de Shepard), resultam em :

$$
\begin{aligned}
& H(x)=\sum_{j=1}^{N} W_{j}\left(x_{j}-x\right)\{1\}\{1\} \Rightarrow H^{-1}(x)=\frac{1}{\sum_{j=1}^{N} W_{j}\left(x_{j}-x\right)} \\
& G_{j}(x)=W_{j}\left(x_{j}-x\right)\{1\}=W_{j}\left(x_{j}-x\right) \\
& \Psi_{j}(x)=p^{T}(x) H^{-1}(x) G_{j}(x)=\frac{W_{j}\left(x_{j}-x\right)}{\sum_{j=1}^{N} W_{j}\left(x_{j}-x\right)}
\end{aligned}
$$

As funções Shepard podem constituir uma PU, pois satisfazem em particular a condição $\sum_{j=1}^{N} \Psi_{j}(x)=1$, ou seja, podem representar de modo exato uma constante. 
Mesmo sabendo que as funções de Shepard são aproximações pobres, estas são muito utilizadas como base inicial para a família das Nuvens "hp". Outro fato importante é que mesmo o aumento do número de variáveis em região de enriquecimento, comparando-se com o emprego de base polinomial inicial de mesma ordem, é compensado pela redução do esforço computacional para a inversão da matriz $\boldsymbol{H}(\boldsymbol{x})$. 


\section{APÊNDICE C - Condição Algébrica}

No capítulo 6 foi utilizado um teorema da álgebra matricial para provar as condições mínimas necessárias para estabilidade do sistema analisado no trabalho de Zienkiewicz et al (1986). Esse teorema é enunciado da seguinte forma:

"Dados uma matriz $\boldsymbol{C}$ de dimensão $\left(\boldsymbol{n}_{\boldsymbol{y}} \boldsymbol{x} \boldsymbol{n}_{x}\right)$, e uma matriz $\boldsymbol{B}$ de dimensão $\left(\boldsymbol{n}_{x} \boldsymbol{x} \boldsymbol{n}_{\boldsymbol{y}}\right)$ a matriz $\boldsymbol{D}$ de dimensão $\left(\boldsymbol{n}_{\boldsymbol{y}} \boldsymbol{x} \boldsymbol{n}_{\boldsymbol{y}}\right)$ resultante da multiplicação de $\boldsymbol{C B}$ só será inversível se $\boldsymbol{n}_{\boldsymbol{x}} \geq \boldsymbol{n}_{\boldsymbol{y}}{ }^{\prime}$.

Para a prova do teorema acima, considere que existe uma matriz $\boldsymbol{E}$ de dimensão $\left(n_{y} \boldsymbol{x} n_{y}\right)$ tal que:

$$
\boldsymbol{D} \cdot \boldsymbol{E}=\boldsymbol{I}
$$

onde $\boldsymbol{I}$ é a matriz identidade de dimensão $\left(\boldsymbol{n}_{\boldsymbol{y}} \boldsymbol{x} \boldsymbol{n}_{\boldsymbol{y}}\right)$.

Considere-se também $\boldsymbol{T C}, \boldsymbol{T B}$ e $\boldsymbol{T D}$ as transformações lineares cujas matrizes relativas as bases canônicas são $\boldsymbol{C}, \boldsymbol{B}$ e $\boldsymbol{E}$.

$$
\begin{gathered}
\boldsymbol{T C}: \mathfrak{R}_{\boldsymbol{n}_{x}} \text { em } \mathfrak{R}_{\boldsymbol{n}_{\boldsymbol{y}}} \\
\boldsymbol{T} \boldsymbol{B}: \mathfrak{R}_{\boldsymbol{n}_{\boldsymbol{y}}} \text { em } \mathfrak{R}_{\boldsymbol{n}_{\boldsymbol{x}}} \\
\boldsymbol{T} \boldsymbol{E}: \mathfrak{R}_{\boldsymbol{n}_{\boldsymbol{y}}} \text { em } \mathfrak{R}_{\boldsymbol{n}_{\boldsymbol{y}}}
\end{gathered}
$$

onde $\mathfrak{R}_{\boldsymbol{n}_{\boldsymbol{x}}}$ são os reais de dimensão $\boldsymbol{n}_{\boldsymbol{x}}$ e $\mathfrak{R}_{\boldsymbol{n}_{\boldsymbol{y}}}$ os reais de dimensão $\boldsymbol{n}_{\boldsymbol{y}}$.

Dessa forma, pode-se escrever a eq.(C.1) da seguinte forma:

$$
T C \cdot(T B E)=I
$$


A eq.(C.5) é a aplicação identidade que por definição é bijetora. Como $I$ é sobrejetora então $\boldsymbol{T C}$ é sobrejetora e, portanto obrigatoriamente, deve-se ter: $\boldsymbol{n}_{\boldsymbol{x}} \geq \boldsymbol{n}_{\boldsymbol{y}}$. 


\section{APÊNDICE D - Desenvolvimento das Matrizes Envolvidas no inf-sup Teste}

No capítulo 8, foi apresentado o inf-sup teste aplicado a FHMT. Neste apêndice serão desenvolvidas todas as matrizes envolvidas na eq.(8.41) para um melhor entendimento sobre o teste numérico proposto. Foram considerados os elementos da figura 8.1 para análise com o inf-sup teste.

Dos elementos propostos na figura 8.1 e sem enriquecimento algum sobre as bases aproximativas envolvidos na FHMT, pode-se escrever:

- Para as tensões no domínio:

$$
\hat{\sigma}=S_{\Omega_{e}} \cdot s_{\Omega}
$$

onde $S_{\Omega_{e}}$ é a matriz que guarda as aproximações para o campo de tensão:

$$
S_{\Omega_{e}}=\left[\begin{array}{cccccccccccc}
\varphi_{1} & 0 & 0 & \varphi_{2} & 0 & 0 & \varphi_{3} & 0 & 0 & \varphi_{4} & 0 & 0 \\
0 & \varphi_{1} & 0 & 0 & \varphi_{2} & 0 & 0 & \varphi_{3} & 0 & 0 & \varphi_{4} & 0 \\
0 & 0 & \varphi_{1} & 0 & 0 & \varphi_{2} & 0 & 0 & \varphi_{3} & 0 & 0 & \varphi_{4}
\end{array}\right]
$$

e $s_{\Omega}$ é o vetor que agrupa os graus de liberdade do campo de tensões no domínio:

$$
s_{\Omega}^{T}=\left\{\begin{array}{llllllllllll}
\sigma_{x_{1}} & \sigma_{y_{1}} & \tau_{x y_{1}} & \sigma_{x_{2}} & \sigma_{y_{2}} & \tau_{x y_{2}} & \sigma_{x_{3}} & \sigma_{y_{3}} & \tau_{x y_{3}} & \sigma_{x_{4}} & \sigma_{y_{4}} & \tau_{x y_{4}}
\end{array}\right\}
$$

- Para os deslocamentos no domínio:

$$
\hat{\boldsymbol{u}}=U_{\Omega_{e}} \cdot q_{\Omega}
$$

onde $\boldsymbol{U}_{\Omega_{e}}$ é a matriz que guarda as aproximações para o campo de deslocamento no domínio: 
$U_{\Omega_{e}}=\left[\begin{array}{cccccccc}\varphi_{1} & 0 & \varphi_{2} & 0 & \varphi_{3} & 0 & \varphi_{4} & 0 \\ 0 & \varphi_{1} & 0 & \varphi_{2} & 0 & \varphi_{3} & 0 & \varphi_{4}\end{array}\right]$

e $\boldsymbol{q}_{\Omega}$ é o vetor que agrupa os graus de liberdade do campo de deslocamentos no domínio:

$q_{\Omega}^{T}=\left[\begin{array}{llllllll}u_{1} & v_{1} & u_{2} & v_{2} & u_{3} & v_{3} & u_{4} & v_{4}\end{array}\right]$

- Para os deslocamentos no contorno:

$$
\hat{\boldsymbol{u}}_{\Gamma}=\boldsymbol{U}_{\Gamma_{i}} \cdot \boldsymbol{q}_{\Gamma}
$$

onde $U_{\Gamma i}$ é a matriz que guarda as aproximações para o campo de deslocamento no contorno:

$\boldsymbol{U}_{\Gamma_{i}}=\left[\begin{array}{cccc}\psi_{1} & 0 & \psi_{2} & 0 \\ 0 & \psi_{1} & 0 & \psi_{2}\end{array}\right]$

e $\boldsymbol{q}_{\Gamma}$ é o vetor que agrupa os graus de liberdade do campo de deslocamentos no domínio:

$\boldsymbol{q}_{\Gamma}=\left\lfloor\begin{array}{llll}u_{\Gamma_{1}} & v_{\Gamma_{1}} & u_{\Gamma_{2}} & v_{\Gamma_{2}}\end{array}\right\rfloor$

O operador diferencial divergente $\boldsymbol{L}$ definido na eq.(2.7) aplicado a eq.(D.2), fornece:

$\boldsymbol{L} \boldsymbol{S}_{\Omega_{e}}=\left[\begin{array}{cccccc}\frac{\partial \varphi_{1}}{\partial \boldsymbol{x}} & 0 & \frac{\partial \varphi_{1}}{\partial \boldsymbol{y}} & \frac{\partial \varphi_{2}}{\partial \boldsymbol{x}} & 0 & \frac{\partial \varphi_{2}}{\partial y} \\ 0 & \frac{\partial \varphi_{1}}{\partial \boldsymbol{y}} & \frac{\partial \varphi_{1}}{\partial \boldsymbol{x}} & 0 & \frac{\partial \varphi_{2}}{\partial y} & \frac{\partial \varphi_{2}}{\partial \boldsymbol{x}}\end{array} \ldots\right.$

$\left.\ldots \begin{array}{cccccc}\frac{\partial \varphi_{3}}{\partial x} & 0 & \frac{\partial \varphi_{3}}{\partial y} & \frac{\partial \varphi_{4}}{\partial x} & 0 & \frac{\partial \varphi_{4}}{\partial y} \\ 0 & \frac{\partial \varphi_{3}}{\partial y} & \frac{\partial \varphi_{3}}{\partial x} & 0 & \frac{\partial \varphi_{4}}{\partial y} & \frac{\partial \varphi_{4}}{\partial x}\end{array}\right]$

A matriz $\boldsymbol{N}$ construída com as componentes do vetor normal ao contorno, definido na eq.(2.10) aplicada a eq.(D.2) fornece: 


$$
\begin{aligned}
& N S_{\Omega_{e}}=\left[\begin{array}{cccccl}
n_{x} \varphi_{1} & 0 & n_{y} \varphi_{1} & n_{x} \varphi_{2} & 0 & n_{y} \varphi_{2} \\
0 & n_{y} \varphi_{1} & n_{x} \varphi_{1} & 0 & n_{y} \varphi_{2} & n_{x} \varphi_{2}
\end{array} \ldots\right. \\
& \left.\begin{array}{cccccc}
n_{x} \varphi_{3} & 0 & n_{y} \varphi_{3} & n_{x} \varphi_{4} & 0 & n_{y} \varphi_{4} \\
0 & n_{y} \varphi_{3} & n_{x} \varphi_{3} & 0 & n_{y} \varphi_{4} & n_{x} \varphi_{4}
\end{array}\right]
\end{aligned}
$$

Da eq.(8.49) e as aproximações definidas nas eq.(D.2), eq.(D.5) e eq.(D.8), escreve-se:

$$
\begin{gathered}
\|U\|_{X}^{2}=\left\|\left(\sigma, u, u_{\Gamma}\right)\right\|_{X}^{2}=s_{\Omega}^{T} \int_{\Omega_{e}} S_{\Omega_{e}}^{T} S_{\Omega_{e}} d \Omega_{e} s_{\Omega}+s_{\Omega}^{T} \int_{\Omega_{e}} S_{\Omega_{e}}^{T} L^{T} L S_{\Omega_{e}} d \Omega_{e} s_{\Omega}+\cdots \\
\cdots+s_{\Omega}^{T} \int_{\Gamma_{t}} S_{\Omega_{e}}^{T} N^{T} N S_{\Omega_{e}} d \Gamma_{i} s_{\Omega}+q_{\Omega}^{T} \int_{\Omega_{e}} U_{\Omega_{e}}^{T} U_{\Omega_{e}} d \Omega_{e} q_{\Omega}+q_{\Gamma_{i}}^{T} \int_{\Gamma_{i}} U_{\Gamma_{i}}^{T} U_{\Gamma_{i}} d \Gamma_{i} q_{\Gamma_{i}}
\end{gathered}
$$

Como definido na eq.(D.30), a eq.(D.12) pode ser escrita da seguinte forma:

$$
\|\boldsymbol{U}\|_{X}^{2}=\mathbf{u}^{\mathrm{T}} A_{I} \mathbf{u}
$$

onde

$$
\mathbf{u}=\left\{\begin{array}{l}
s_{V} \\
\boldsymbol{q}_{V} \\
\boldsymbol{q}_{\Gamma}
\end{array}\right\}
$$

e

$$
\begin{aligned}
& A_{1}=\left[\begin{array}{c}
\int_{\Omega_{e}} S_{\Omega_{e}}^{T} S_{\Omega_{e}} d \Omega_{e}+\int_{\Omega_{e}} S_{\Omega_{e}}^{T} L^{T} L S_{\Omega_{e}} d \Omega_{e}+\int_{\Gamma_{t}} S_{\Omega_{e}}^{T} N^{T} N S_{\Omega_{e}} d \Gamma_{i} \\
\mathrm{O}_{1}^{T} \\
\mathrm{O}_{2}^{T}
\end{array} \ldots\right. \\
& \left.\begin{array}{cc}
O_{1} & O_{2} \\
\cdots \int_{\Omega_{e}} U_{\Omega_{e}}^{T} U_{\Omega_{e}} d \Omega_{e} & O_{3} \\
O_{3}^{T} & \int_{\Omega_{e}} U_{\Gamma_{i}}^{T} U_{\Gamma_{i}} d \Gamma_{i}
\end{array}\right]
\end{aligned}
$$


Na eq.(D.16) $O_{1}$ de dimensão $(12 x \quad 8), O_{2}$ de dimensão $\left(\begin{array}{llll}12 & x & 4\end{array}\right)$ e $O_{3}$ de dimensão $\left(\begin{array}{lll}8 & x & 4\end{array}\right)$ são matrizes nulas. Das eq.(D.2), eq.(D.5), eq.(D.8), eq.(D.10) e eq.(D.11), pode-se concluir que a dimensão da matriz $\boldsymbol{A}_{1}$ eq.(D.15) é de (24 x 24).

Da eq.(8.31), tem-se que matriz $\boldsymbol{B}(\boldsymbol{U}, \boldsymbol{V})$ dada pela eq.(8.45), pode ser escrita da seguinte forma:

$$
B(U, V)=\mathbf{v}^{\mathrm{T}} B \mathbf{u}
$$

onde

$$
\mathbf{v}^{\mathbf{T}}=\left\{\begin{array}{lll}
\delta \mathbf{s}_{\Omega}^{T} & \delta \boldsymbol{q}_{\Omega}^{T} & \delta \boldsymbol{q}_{\Gamma}^{T}
\end{array}\right\}
$$

$\mathrm{e}$

$$
\boldsymbol{B}=\left[\begin{array}{ccc}
\boldsymbol{F} & \boldsymbol{A}_{\Omega} & -\boldsymbol{A}_{\Gamma} \\
\boldsymbol{A}_{\Omega}^{T} & \boldsymbol{0} & \boldsymbol{0} \\
-\boldsymbol{A}_{\Gamma}^{T} & \boldsymbol{0} & \boldsymbol{0}
\end{array}\right]
$$

onde foram introduzidas as seguintes matrizes:

$$
\begin{aligned}
& F=\int_{\Omega} S_{\Omega}^{T} f S_{\Omega} d \Omega \\
& A_{\Omega}=\int_{\Omega}\left(L S_{\Omega}\right)^{T} U_{\Omega} d \Omega \\
& A_{\Gamma}=\int_{\Gamma_{t}}\left(N S_{\Omega}\right)^{T} U_{\Gamma} d \Gamma
\end{aligned}
$$

Para o elemento proposto, sem enriquecimento, tem-se que a dimensão da matriz $\boldsymbol{B}$ dada pela eq.(D.18) é (24 $\times 24)$.

Como pode ser observado no desenvolvimento das equações deste apêndice, o espaço das funções peso $\boldsymbol{V}$ é o mesmo das funções teste $\boldsymbol{U}$ e por isso $\boldsymbol{A}_{2}=\boldsymbol{A}_{\boldsymbol{1}}$. Assim, estão definidos todos os parâmetros da eq.(8.41) necessários para o cálculo de $\lambda(\boldsymbol{n})$.

Agora, para a determinação de $\boldsymbol{A}_{\boldsymbol{1}}$ e $\boldsymbol{B}$ dentro das várias condições de enriquecimento é só ampliar da forma desejada as bases aproximativas iniciais, atrelada a cada nó do elemento, dos campos de tensão e deslocamento no domínio e deslocamento no contorno. 


\section{APÊNDICE E - Enriquecimento Nodal com Soluções da Mecânica da Fratura Elástica Linear}

Considere o elemento finito quadrilateral de quatro nós apresentado na figura 5.3 e as mesmas funções aproximativas para os campos envolvidos na FHMT. Para esses elementos pode-se escrever:

- Matriz que guardam as aproximações para o campo de tensão:

$$
S_{\Omega_{e}}=\left[\begin{array}{cccccccccccc}
\varphi_{1} & 0 & 0 & \varphi_{2} & 0 & 0 & \varphi_{3} & 0 & 0 & \varphi_{4} & 0 & 0 \\
0 & \varphi_{1} & 0 & 0 & \varphi_{2} & 0 & 0 & \varphi_{3} & 0 & 0 & \varphi_{4} & 0 \\
0 & 0 & \varphi_{1} & 0 & 0 & \varphi_{2} & 0 & 0 & \varphi_{3} & 0 & 0 & \varphi_{4}
\end{array}\right]
$$

- Matriz que guardam as aproximações para o campo de deslocamento no domínio:

$$
U_{\Omega_{e}}=\left[\begin{array}{cccccccc}
\varphi_{1} & 0 & \varphi_{2} & 0 & \varphi_{3} & 0 & \varphi_{4} & 0 \\
0 & \varphi_{1} & 0 & \varphi_{2} & 0 & \varphi_{3} & 0 & \varphi_{4}
\end{array}\right]
$$

- Matriz que guardam as aproximações para o campo de deslocamento no contorno:

$$
\boldsymbol{U}_{\Gamma_{i}}=\left[\begin{array}{cccc}
\psi_{1} & 0 & \psi_{2} & 0 \\
0 & \psi_{1} & 0 & \psi_{2}
\end{array}\right]
$$

Com a possibilidade de enriquecimento de todos os nós dos elementos finitos da figura (6.1) com funções que se aproximam das tensões elásticas (eq.(5.37), eq.(5.38) e eq.(5.39)) e do campo de deslocamentos (eq.(5.40) e eq.(5.41)) próximo à ponta da fissura representada na figura (5.2), as eq.(E.1) e eq(E.2) tem a seguinte apresentação: 


$$
\begin{aligned}
& \boldsymbol{S}_{\Omega_{e}}=\left[\begin{array}{cccccc}
\varphi_{1} & \mathbf{0} & \mathbf{0} & \varphi_{1}\left(\sigma_{x}^{\prime}\right) & \mathbf{0} & \mathbf{0} \\
\mathbf{0} & \varphi_{1} & \mathbf{0} & \mathbf{0} & \varphi_{1}\left(\sigma_{y}^{\prime}\right) & \mathbf{0}
\end{array} \ldots\right. \\
& \begin{array}{llllll}
\varphi_{2} & \mathbf{0} & \mathbf{0} & \varphi_{2}\left(\sigma_{x}^{\prime}\right) & \mathbf{0} & \mathbf{0}
\end{array}
\end{aligned}
$$

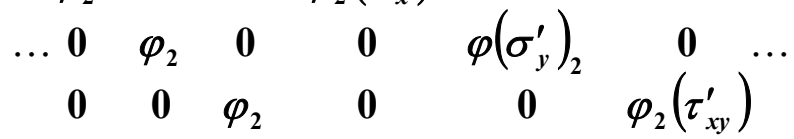

$$
\begin{aligned}
& \begin{array}{cccccc}
\varphi_{3} & \mathbf{0} & \mathbf{0} & \varphi_{3}\left(\sigma_{x}^{\prime}\right) & \mathbf{0} & \mathbf{0}
\end{array} \\
& \begin{array}{rcccccc}
\ldots & \mathbf{0} & \varphi_{3} & \mathbf{0} & \mathbf{0} & \varphi_{3}\left(\sigma_{y}^{\prime}\right) & \mathbf{0}
\end{array} \ldots \\
& \left.\begin{array}{rccccc}
\varphi_{4} & \mathbf{0} & \mathbf{0} & \varphi_{4}\left(\sigma_{x}^{\prime}\right) & \mathbf{0} & \mathbf{0} \\
\cdots \mathbf{0} & \varphi_{4} & \mathbf{0} & \mathbf{0} & \varphi_{4}\left(\sigma_{y}^{\prime}\right) & \mathbf{0} \\
\mathbf{0} & \mathbf{0} & \varphi_{4} & \mathbf{0} & \mathbf{0} & \varphi_{4}\left(\tau_{x y}^{\prime}\right)
\end{array}\right]
\end{aligned}
$$

Observa-se que a dimensão da matriz $S_{\Omega_{e}}$ passou de (3 x 12) para (3 x 24), quando todos os nós são enriquecidos.

$$
\begin{aligned}
& \boldsymbol{U}_{\Omega_{e}}=\left[\begin{array}{cccccccc}
\varphi_{1} & \mathbf{0} & \varphi_{1}\left(\boldsymbol{u}^{\prime}\right) & \mathbf{0} & \varphi_{2} & \mathbf{0} & \varphi_{2}\left(\boldsymbol{u}^{\prime}\right) & \mathbf{0} \\
\mathbf{0} & \varphi_{1} & \mathbf{0} & \varphi_{1}\left(v^{\prime}\right) & \mathbf{0} & \varphi_{2} & \mathbf{0} & \varphi_{2}\left(v^{\prime}\right)
\end{array} \ldots\right. \\
& \left.\begin{array}{cccccccc}
\varphi_{3} & \mathbf{0} & \varphi_{3}\left(\boldsymbol{u}^{\prime}\right) & \mathbf{0} & \varphi_{4} & \mathbf{0} & \varphi_{4}\left(\boldsymbol{u}^{\prime}\right) & \mathbf{0} \\
\mathbf{0} & \varphi_{3} & \mathbf{0} & \varphi_{3}\left(v^{\prime}\right) & \mathbf{0} & \varphi_{4} & \mathbf{0} & \varphi_{4}\left(v^{\prime}\right)
\end{array}\right]
\end{aligned}
$$

Observa-se que a dimensão da matriz $\boldsymbol{U}_{\Omega_{e}}$ passou de $(2 \times 8)$ para $(2 \times 16)$, quando todos os nós são enriquecidos.

A matriz $\boldsymbol{F}_{e}$, dada pela eq.(4.11), terá uma dimensão de (24 x 24), com todos os nós enriquecidos, como mostra a eq.(E.6). 
Linha 1,2 e 3 da matriz $\boldsymbol{F}_{e}$ :

$$
\begin{aligned}
& {\left[\begin{array}{cccccc}
\varphi_{1} f_{11} \varphi_{1} & \varphi_{1} f_{12} \varphi_{1} & 0 & \varphi_{1} f_{11}\left(\varphi_{1} \sigma_{x}^{\prime}\right) & \varphi_{1} f_{12}\left(\varphi_{1} \sigma_{y}^{\prime}\right) & 0 \\
\varphi_{1} f_{21} \varphi_{1} & \varphi_{1} f_{22} \varphi_{1} & 0 & \varphi_{1} f_{21}\left(\varphi_{1} \sigma_{x}^{\prime}\right) & \varphi_{1} f_{22}\left(\varphi_{1} \sigma_{y}^{\prime}\right) & 0
\end{array}\right] \ldots} \\
& \begin{array}{lllll}
\varphi_{1} f_{11} \varphi_{2} & \varphi_{1} f_{12} \varphi_{2} & 0 & \varphi_{1} f_{11}\left(\varphi_{2} \sigma_{x}^{\prime}\right) & \varphi_{1} f_{12}\left(\varphi_{2} \sigma_{y}^{\prime}\right)
\end{array} \\
& \begin{array}{ccccc}
\cdots \varphi_{1} f_{21} \varphi_{2} & \varphi_{1} f_{22} \varphi_{2} & 0 & \varphi_{1} f_{21}\left(\varphi_{2} \sigma_{x}^{\prime}\right) & \varphi_{1} f_{22}\left(\varphi_{2} \sigma_{y}^{\prime}\right)
\end{array} \\
& \begin{array}{cccccc}
\mathbf{0} & \mathbf{0} & \varphi_{1} f_{33} \varphi_{2} & \mathbf{0} & \mathbf{0} & \varphi_{1} f_{33}\left(\varphi_{2} \tau_{x y}^{\prime}\right)
\end{array}
\end{aligned}
$$

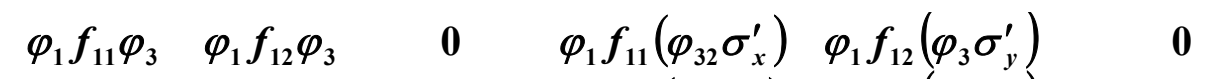

$\begin{array}{cccccc}\cdots \varphi_{1} f_{21} \varphi_{3} & \varphi_{1} f_{22} \varphi_{3} & 0 & \varphi_{1} f_{21}\left(\varphi_{3} \sigma_{x}^{\prime}\right) & \varphi_{1} f_{22}\left(\varphi_{3} \sigma_{y}^{\prime}\right) & 0 \\ 0 & 0 & \varphi_{1} f_{33} \varphi_{3} & 0 & 0 & \varphi_{1} f_{33}\left(\varphi_{3} \tau_{x y}^{\prime}\right)\end{array}{ }^{0}$

$$
\left.\begin{array}{cccccc}
\varphi_{1} f_{11} \varphi_{4} & \varphi_{1} f_{12} \varphi_{4} & 0 & \varphi_{1} f_{11}\left(\varphi_{4} \sigma_{x}^{\prime}\right) & \varphi_{1} f_{12}\left(\varphi_{4} \sigma_{y}^{\prime}\right) & 0 \\
\cdots \varphi_{1} f_{21} \varphi_{4} & \varphi_{1} f_{22} \varphi_{4} & 0 & \varphi_{1} f_{21}\left(\varphi_{4} \sigma_{x}^{\prime}\right) & \varphi_{1} f_{22}\left(\varphi_{4} \sigma_{y}^{\prime}\right) & 0 \\
0 & 0 & \varphi_{1} f_{33} \varphi_{4} & 0 & 0 & \varphi_{1} f_{33}\left(\varphi_{4} \tau_{x y}^{\prime}\right)
\end{array}\right]
$$

Linhas 4, 5 e 6 da matriz $\boldsymbol{F}_{e}$ :

$\left[\begin{array}{cccccc}\left(\varphi_{1} \sigma_{x}^{\prime}\right) f_{1} \varphi_{1} & \left(\varphi_{1} \sigma_{y}^{\prime}\right) f_{12} \varphi_{1} & \mathbf{0} & \left(\varphi_{1} \sigma_{x}^{\prime}\right) f_{11}\left(\varphi_{1} \sigma_{x}^{\prime}\right) & \left(\varphi_{1} \sigma_{y}^{\prime}\right) f_{12}\left(\varphi_{1} \sigma_{y}^{\prime}\right) & \mathbf{0} \\ \left(\varphi_{1} \sigma_{x}^{\prime}\right) f_{21} \varphi_{1} & \left(\varphi_{1} \sigma_{y}^{\prime}\right) f_{22} \varphi_{1} & \mathbf{0} & \left(\varphi_{1} \sigma_{x}^{\prime}\right) f_{21}\left(\varphi_{1} \sigma_{x}^{\prime}\right) & \left(\varphi_{1} \sigma_{y}^{\prime}\right) f_{22}\left(\varphi_{1} \sigma_{y}^{\prime}\right) & \mathbf{0} \\ \mathbf{0} & \mathbf{0} & \left(\varphi_{1} \tau_{x y}^{\prime}\right) f_{33} \varphi_{1} & \mathbf{0} & \mathbf{0} & \left(\varphi_{1} \tau_{x y}^{\prime}\right) f_{33}\left(\varphi_{1} \tau_{x y}^{\prime}\right)\end{array}\right.$

$$
\left.\begin{array}{cccccc}
\left(\varphi_{1} \sigma_{x}^{\prime}\right) f_{11} \varphi_{2} & \left(\varphi_{1} \sigma_{y}^{\prime}\right) f_{12} \varphi_{2} & 0 & \left(\varphi_{1} \sigma_{x}^{\prime}\right) f_{11}\left(\varphi_{2} \sigma_{x}^{\prime}\right) & \left(\varphi_{1} \sigma_{y}^{\prime}\right) f_{12}\left(\varphi_{2} \sigma_{y}^{\prime}\right) & \mathbf{0} \\
\cdots\left(\varphi_{1} \sigma_{x}^{\prime}\right) f_{21} \varphi_{2} & \left(\varphi_{1} \sigma_{y}^{\prime}\right) f_{22} \varphi_{2} & \mathbf{0} & \left(\varphi_{1} \sigma_{x}^{\prime}\right) f_{21}\left(\varphi_{2} \sigma_{x}^{\prime}\right) & \left(\varphi_{1} \sigma_{y}^{\prime}\right) f_{22}\left(\varphi_{2} \sigma_{y}^{\prime}\right) & \mathbf{0}
\end{array}\right]
$$


$\left.\begin{array}{cccccc}\left(\varphi_{1} \sigma_{x}^{\prime}\right) f_{11} \varphi_{3} & \left(\varphi_{1} \sigma_{y}^{\prime}\right) f_{12} \varphi_{3} & \mathbf{0} & \left(\varphi_{1} \sigma_{x}^{\prime}\right) f_{11}\left(\varphi_{32} \sigma_{x}^{\prime}\right) & \left(\varphi_{1} \sigma_{y}^{\prime}\right) f_{12}\left(\varphi_{3} \sigma_{y}^{\prime}\right) & \mathbf{0} \\ \cdots\left(\varphi_{1} \sigma_{x}^{\prime}\right) f_{21} \varphi_{3} & \left(\varphi_{1} \sigma_{y}^{\prime}\right) f_{22} \varphi_{3} & \mathbf{0} & \left(\varphi_{1} \sigma_{x}^{\prime}\right) f_{21}\left(\varphi_{3} \sigma_{x}^{\prime}\right) & \left(\varphi_{1} \sigma_{y}^{\prime}\right) f_{22}\left(\varphi_{3} \sigma_{y}^{\prime}\right) & \mathbf{0} \\ \mathbf{0} & \mathbf{0} & \left(\varphi_{1} \tau_{x y}^{\prime}\right) f_{33} \varphi_{3} & \mathbf{0} & \mathbf{0} & \left(\varphi_{1} \tau_{x y}^{\prime}\right) f_{33}\left(\varphi_{3} \tau_{x y}^{\prime}\right) \\ & & & & & \\ \left(\varphi_{1} \sigma_{x}^{\prime}\right) f_{11} \varphi_{4} & \left(\varphi_{1} \sigma_{y}^{\prime}\right) f_{12} \varphi_{4} & \mathbf{0} & \left(\varphi_{1} \sigma_{x}^{\prime}\right) f_{11}\left(\varphi_{4} \sigma_{x}^{\prime}\right) & \left(\varphi_{1} \sigma_{y}^{\prime}\right) f_{12}\left(\varphi_{4} \sigma_{y}^{\prime}\right) & \mathbf{0} \\ \cdots\left(\varphi_{1} \sigma_{x}^{\prime}\right) f_{21} \varphi_{4} & \left(\varphi_{1} \sigma_{y}^{\prime}\right) f_{22} \varphi_{4} & \mathbf{0} & \left(\varphi_{1} \sigma_{x}^{\prime}\right) f_{21}\left(\varphi_{4} \sigma_{x}^{\prime}\right) & \left(\varphi_{1} \sigma_{y}^{\prime}\right) f_{22}\left(\varphi_{4} \sigma_{y}^{\prime}\right) & \mathbf{0} \\ \mathbf{0} & \mathbf{0} & \left(\varphi_{1} \tau_{x y}^{\prime}\right) f_{33} \varphi_{4} & \mathbf{0} & \mathbf{0} & \left(\varphi_{1} \tau_{x y}^{\prime}\right) f_{33}\left(\varphi_{4} \tau_{x y}^{\prime}\right)\end{array}\right]$

Linhas 7, 8 e 9 da matriz $\boldsymbol{F}_{\boldsymbol{e}}$ :

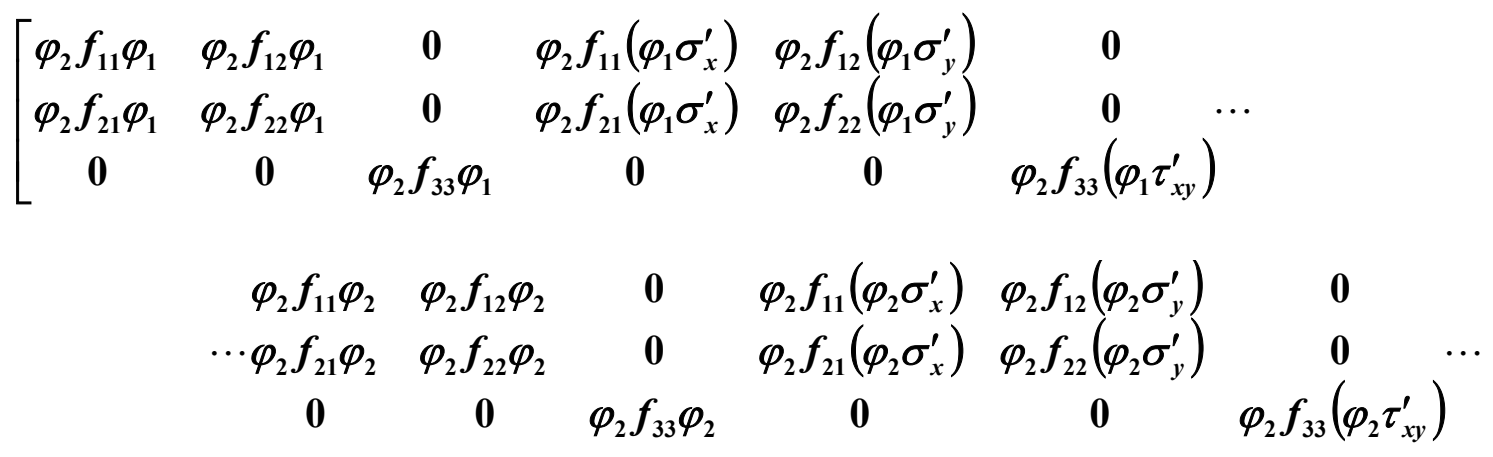

$\varphi_{2} f_{11} \varphi_{3} \quad \varphi_{2} f_{12} \varphi_{3} \quad 0 \quad \varphi_{2} f_{11}\left(\varphi_{32} \sigma_{x}^{\prime}\right) \quad \varphi_{2} f_{12}\left(\varphi_{3} \sigma_{y}^{\prime}\right) \quad$ 0

$\left.\begin{array}{cccccc}\cdots \varphi_{2} f_{21} \varphi_{3} & \varphi_{2} f_{22} \varphi_{3} & \mathbf{0} & \varphi_{2} f_{21}\left(\varphi_{3} \sigma_{x}^{\prime}\right) & \varphi_{2} f_{22}\left(\varphi_{3} \sigma_{y}^{\prime}\right) & \mathbf{0}\end{array}\right]$

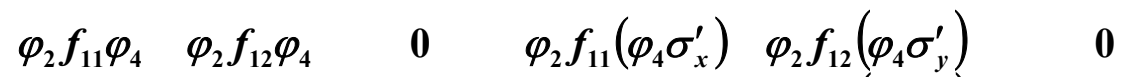

$\left.\begin{array}{cccccc}\cdots \varphi_{2} f_{21} \varphi_{4} & \varphi_{2} f_{22} \varphi_{4} & \mathbf{0} & \varphi_{2} f_{21}\left(\varphi_{4} \sigma_{x}^{\prime}\right) & \varphi_{2} f_{22}\left(\varphi_{4} \sigma_{y}^{\prime}\right) & \mathbf{0} \\ \mathbf{0} & \mathbf{0} & \varphi_{2} f_{33} \varphi_{4} & \mathbf{0} & \mathbf{0} & \varphi_{2} f_{33}\left(\varphi_{4} \tau_{x y}^{\prime}\right)\end{array}\right]$

Linhas 10,11 e 12 da matriz $\boldsymbol{F}_{\boldsymbol{e}}$ :

$\left[\begin{array}{cccccc}\left(\varphi_{2} \sigma_{x}^{\prime}\right) f_{11} \varphi_{1} & \left(\varphi_{2} \sigma_{y}^{\prime}\right) f_{12} \varphi_{1} & \mathbf{0} & \left(\varphi_{2} \sigma_{x}^{\prime}\right) f_{11}\left(\varphi_{1} \sigma_{x}^{\prime}\right) & \left(\varphi_{2} \sigma_{y}^{\prime}\right) f_{12}\left(\varphi_{1} \sigma_{y}^{\prime}\right) & \mathbf{0} \\ \left(\varphi_{2} \sigma_{x}^{\prime}\right) f_{21} \varphi_{1} & \left(\varphi_{2} \sigma_{y}^{\prime}\right) f_{22} \varphi_{1} & \mathbf{0} & \left(\varphi_{2} \sigma_{x}^{\prime}\right) f_{21}\left(\varphi_{1} \sigma_{x}^{\prime}\right) & \left(\varphi_{2} \sigma_{y}^{\prime}\right) f_{22}\left(\varphi_{1} \sigma_{y}^{\prime}\right) & \mathbf{0}\end{array}\right]$ 


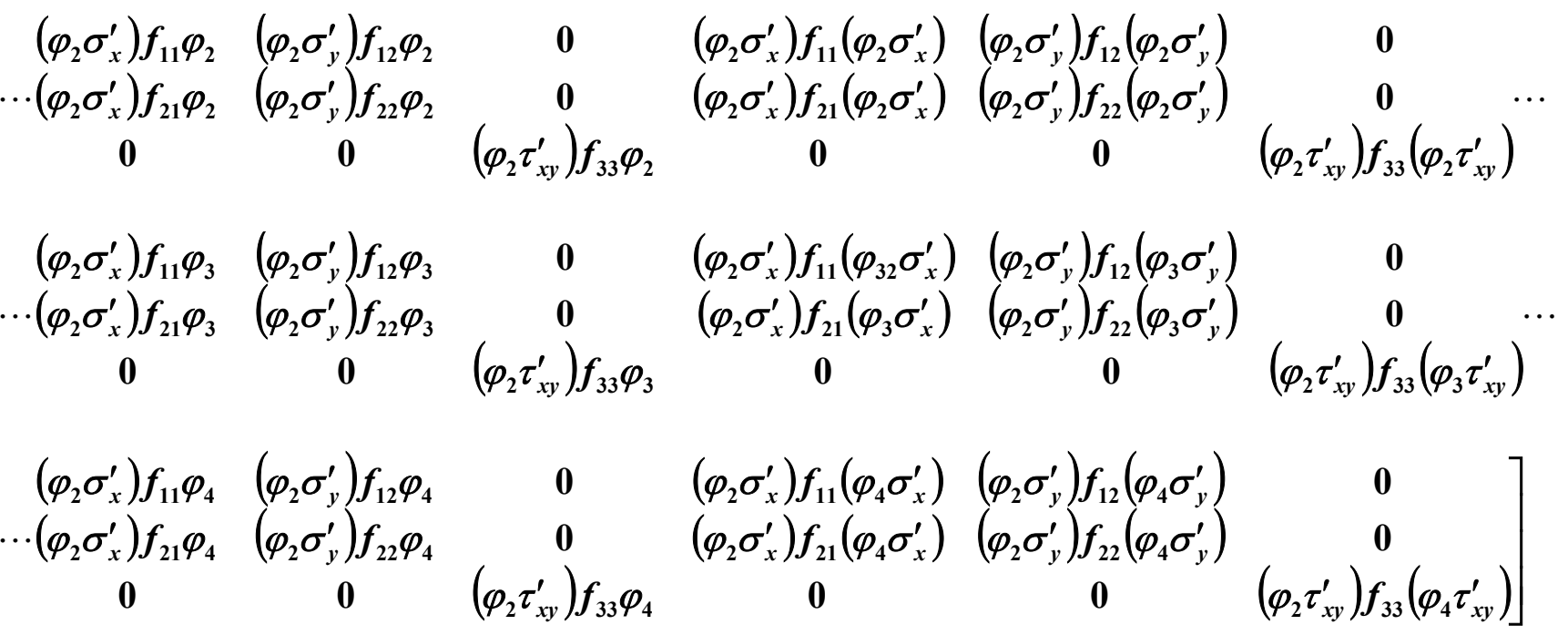

Linhas 13,14 e 15 da matriz $\boldsymbol{F}_{e}$ :

$$
\begin{aligned}
& {\left[\begin{array}{cccccc}
\varphi_{3} f_{11} \varphi_{1} & \varphi_{3} f_{12} \varphi_{1} & \mathbf{0} & \varphi_{3} f_{11}\left(\varphi_{1} \sigma_{x}^{\prime}\right) & \varphi_{3} f_{12}\left(\varphi_{1} \sigma_{y}^{\prime}\right) & \mathbf{0} \\
\varphi_{3} f_{21} \varphi_{1} & \varphi_{3} f_{22} \varphi_{1} & \mathbf{0} & \varphi_{3} f_{21}\left(\varphi_{1} \sigma_{x}^{\prime}\right) & \varphi_{3} f_{22}\left(\varphi_{1} \sigma_{y}^{\prime}\right) & \mathbf{0} \\
\mathbf{0} & \mathbf{0} & \varphi_{3} f_{33} \varphi_{1} & \mathbf{0} & \mathbf{0} & \varphi_{3} f_{33}\left(\varphi_{1} \tau_{x y}^{\prime}\right)
\end{array} \ldots\right.} \\
& \varphi_{3} f_{11} \varphi_{2} \quad \varphi_{3} f_{12} \varphi_{2} \quad 0 \quad \varphi_{3} f_{11}\left(\varphi_{2} \sigma_{x}^{\prime}\right) \quad \varphi_{3} f_{12}\left(\varphi_{2} \sigma_{y}^{\prime}\right) \quad \mathbf{0} \\
& \left.\begin{array}{cccccc}
\cdots \varphi_{3} f_{21} \varphi_{2} & \varphi_{3} f_{22} \varphi_{2} & \mathbf{0} & \varphi_{3} f_{21}\left(\varphi_{2} \sigma_{x}^{\prime}\right) & \varphi_{3} f_{22}\left(\varphi_{2} \sigma_{y}^{\prime}\right) & \mathbf{0}
\end{array}\right]
\end{aligned}
$$


Linhas 16,17 e 18 da matriz $\boldsymbol{F}_{e}$ :

$$
\begin{aligned}
& {\left[\begin{array}{cccccc}
\left(\varphi_{3} \sigma_{x}^{\prime}\right) f_{11} \varphi_{1} & \left(\varphi_{3} \sigma_{y}^{\prime}\right) f_{12} \varphi_{1} & \mathbf{0} & \left(\varphi_{3} \sigma_{x}^{\prime}\right) f_{11}\left(\varphi_{3} \sigma_{x}^{\prime}\right) & \left(\varphi_{3} \sigma_{y}^{\prime}\right) f_{12}\left(\varphi_{1} \sigma_{y}^{\prime}\right) & \mathbf{0} \\
\left(\varphi_{3} \sigma_{x}^{\prime}\right) f_{21} \varphi_{1} & \left(\varphi_{3} \sigma_{y}^{\prime}\right) f_{22} \varphi_{1} & \mathbf{0} & \left(\varphi_{3} \sigma_{x}^{\prime}\right) f_{21}\left(\varphi_{3} \sigma_{x}^{\prime}\right) & \left(\varphi_{3} \sigma_{y}^{\prime}\right) f_{22}\left(\varphi_{1} \sigma_{y}^{\prime}\right) & \mathbf{0}
\end{array}\right]}
\end{aligned}
$$

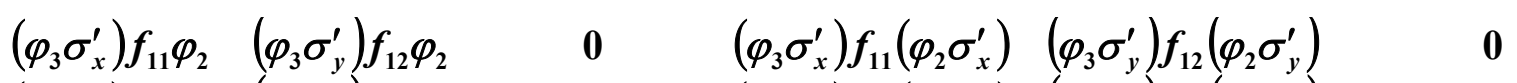

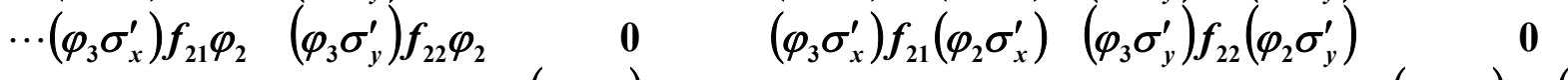

$$
\begin{aligned}
& \begin{array}{cccccc}
\mathbf{0} & \mathbf{0} & \left(\varphi_{3} \tau_{x y}^{\prime}\right) f_{33} \varphi_{2} & \mathbf{0} & \mathbf{0} & \left(\varphi_{3} \tau_{x y}^{\prime}\right) f_{33}\left(\varphi_{2} \tau_{x y}^{\prime}\right)
\end{array} \\
& \left(\varphi_{3} \sigma_{x}^{\prime}\right) f_{11} \varphi_{3} \quad\left(\varphi_{3} \sigma_{y}^{\prime}\right) f_{12} \varphi_{3} \quad \mathbf{0} \quad\left(\varphi_{3} \sigma_{x}^{\prime}\right) f_{11}\left(\varphi_{32} \sigma_{x}^{\prime}\right)\left(\varphi_{3} \sigma_{y}^{\prime}\right) f_{12}\left(\varphi_{3} \sigma_{y}^{\prime}\right) \quad \text { 0 }
\end{aligned}
$$

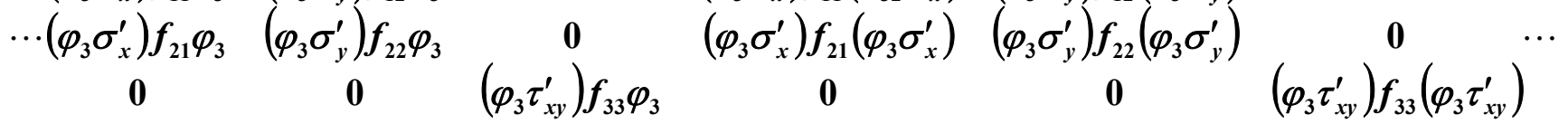

$$
\begin{aligned}
& \begin{array}{llllll}
\left(\varphi_{3} \sigma_{x}^{\prime}\right) f_{11} \varphi_{4} & \left(\varphi_{3} \sigma_{y}^{\prime}\right) f_{12} \varphi_{4} & \mathbf{0} & \left(\varphi_{3} \sigma_{x}^{\prime}\right) f_{11}\left(\varphi_{4} \sigma_{x}^{\prime}\right) & \left(\varphi_{3} \sigma_{y}^{\prime}\right) f_{12}\left(\varphi_{4} \sigma_{y}^{\prime}\right) & \mathbf{0} \\
\left(\varphi_{3} \sigma_{x}^{\prime}\right) f_{21} \varphi_{4} & \left(\varphi_{3} \sigma_{y}^{\prime}\right) f_{22} \varphi_{4} & \mathbf{0} & \left(\varphi_{3} \sigma_{x}^{\prime}\right) f_{21}\left(\varphi_{4} \sigma_{x}^{\prime}\right) & \left(\varphi_{3} \sigma_{y}^{\prime}\right) f_{22}\left(\varphi_{4} \sigma_{y}^{\prime}\right) & \mathbf{0}
\end{array} \\
& \begin{array}{cccccc}
\mathbf{0} & \mathbf{0} & \left(\varphi_{3} \tau_{x y}^{\prime}\right) f_{33} \varphi_{4} & \mathbf{0} & \mathbf{0} & \left.\left(\varphi_{3} \tau_{x y}^{\prime}\right) f_{33}\left(\varphi_{4} \tau_{x y}^{\prime}\right)\right]
\end{array}
\end{aligned}
$$

Linhas 19, 20 e 21 da matriz $\boldsymbol{F}_{\boldsymbol{e}}$ :

$$
\begin{aligned}
& {\left[\begin{array}{cccccc}
\varphi_{4} f_{11} \varphi_{1} & \varphi_{4} f_{12} \varphi_{1} & \mathbf{0} & \varphi_{4} f_{11}\left(\varphi_{1} \sigma_{x}^{\prime}\right) & \varphi_{4} f_{12}\left(\varphi_{1} \sigma_{y}^{\prime}\right) & \mathbf{0} \\
\varphi_{4} f_{21} \varphi_{1} & \varphi_{4} f_{22} \varphi_{1} & \mathbf{0} & \varphi_{4} f_{21}\left(\varphi_{1} \sigma_{x}^{\prime}\right) & \varphi_{4} f_{22}\left(\varphi_{1} \sigma_{y}^{\prime}\right) & \mathbf{0}
\end{array}\right]} \\
& \left.\begin{array}{cccccc}
\varphi_{4} f_{11} \varphi_{2} & \varphi_{4} f_{12} \varphi_{2} & \mathbf{0} & \varphi_{4} f_{11}\left(\varphi_{2} \sigma_{x}^{\prime}\right) & \varphi_{4} f_{12}\left(\varphi_{2} \sigma_{y}^{\prime}\right) & \mathbf{0} \\
\cdots \varphi_{4} f_{21} \varphi_{2} & \varphi_{4} f_{22} \varphi_{2} & \mathbf{0} & \varphi_{4} f_{21}\left(\varphi_{2} \sigma_{x}^{\prime}\right) & \varphi_{4} f_{22}\left(\varphi_{2} \sigma_{y}^{\prime}\right) & \mathbf{0}
\end{array}\right]
\end{aligned}
$$

$\varphi_{4} f_{11} \varphi_{3} \quad \varphi_{4} f_{12} \varphi_{3} \quad 0 \quad \varphi_{4} f_{11}\left(\varphi_{32} \sigma_{x}^{\prime}\right) \quad \varphi_{4} f_{12}\left(\varphi_{3} \sigma_{y}^{\prime}\right) \quad \mathbf{0}$

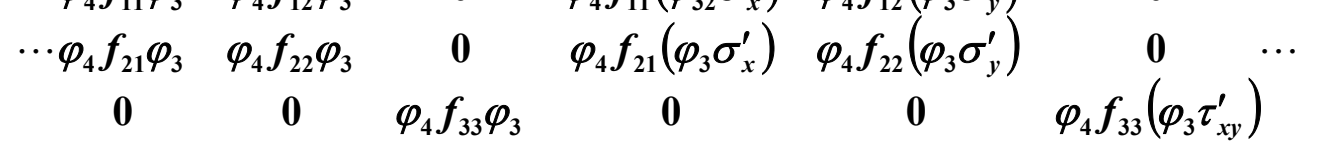




$$
\left.\begin{array}{cccccc}
\varphi_{4} f_{11} \varphi_{4} & \varphi_{4} f_{12} \varphi_{4} & 0 & \varphi_{4} f_{11}\left(\varphi_{4} \sigma_{x}^{\prime}\right) & \varphi_{4} f_{12}\left(\varphi_{4} \sigma_{y}^{\prime}\right) & 0 \\
\cdots \varphi_{4} f_{21} \varphi_{4} & \varphi_{4} f_{22} \varphi_{4} & 0 & \varphi_{4} f_{21}\left(\varphi_{4} \sigma_{x}^{\prime}\right) & \varphi_{4} f_{22}\left(\varphi_{4} \sigma_{y}^{\prime}\right) & 0 \\
0 & 0 & \varphi_{4} f_{33} \varphi_{4} & 0 & 0 & \varphi_{4} f_{33}\left(\varphi_{4} \tau_{x y}^{\prime}\right)
\end{array}\right]
$$

Linhas 22,23 e 24 da matriz $\boldsymbol{F}_{\boldsymbol{e}}$ :

$\left[\begin{array}{cccccc}\left(\varphi_{4} \sigma_{x}^{\prime}\right) f_{11} \varphi_{1} & \left(\varphi_{4} \sigma_{y}^{\prime}\right) f_{12} \varphi_{1} & 0 & \left(\varphi_{4} \sigma_{x}^{\prime}\right) f_{11}\left(\varphi_{3} \sigma_{x}^{\prime}\right) & \left(\varphi_{4} \sigma_{y}^{\prime}\right) f_{12}\left(\varphi_{1} \sigma_{y}^{\prime}\right) & 0 \\ \left(\varphi_{4} \sigma_{x}^{\prime}\right) f_{21} \varphi_{1} & \left(\varphi_{4} \sigma_{y}^{\prime}\right) f_{22} \varphi_{1} & \mathbf{0} & \left(\varphi_{4} \sigma_{x}^{\prime}\right) f_{21}\left(\varphi_{3} \sigma_{x}^{\prime}\right) & \left(\varphi_{4} \sigma_{y}^{\prime}\right) f_{22}\left(\varphi_{1} \sigma_{y}^{\prime}\right) & \mathbf{0}\end{array}\right] \ldots$

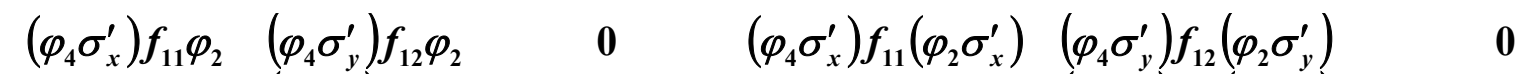

$\begin{array}{lllll}\cdots\left(\varphi_{4} \sigma_{x}^{\prime}\right) f_{21} \varphi_{2} & \left(\varphi_{4} \sigma_{y}^{\prime}\right) f_{22} \varphi_{2} & \mathbf{0} & \left(\varphi_{4} \sigma_{x}^{\prime}\right) f_{21}\left(\varphi_{2} \sigma_{x}^{\prime}\right) & \left(\varphi_{4} \sigma_{y}^{\prime}\right) f_{22}\left(\varphi_{2} \sigma_{y}^{\prime}\right)\end{array}$

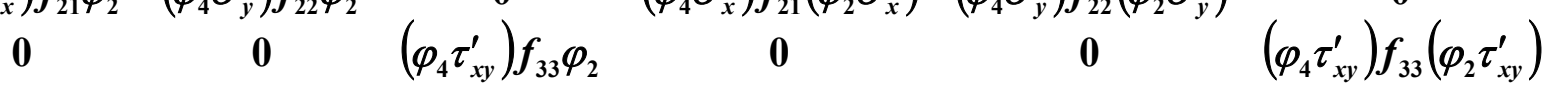

$\begin{array}{cccccc}\left(\varphi_{4} \sigma_{x}^{\prime}\right) f_{11} \varphi_{3} & \left(\varphi_{4} \sigma_{y}^{\prime}\right) f_{12} \varphi_{3} & \mathbf{0} & \left(\varphi_{4} \sigma_{x}^{\prime}\right) f_{11}\left(\varphi_{32} \sigma_{x}^{\prime}\right) & \left(\varphi_{4} \sigma_{y}^{\prime}\right) f_{12}\left(\varphi_{3} \sigma_{y}^{\prime}\right) & \mathbf{0} \\ \cdots\left(\varphi_{4} \sigma_{x}^{\prime}\right) f_{21} \varphi_{3} & \left(\varphi_{4} \sigma_{y}^{\prime}\right) f_{22} \varphi_{3} & \mathbf{0} & \left(\varphi_{4} \sigma_{x}^{\prime}\right) f_{21}\left(\varphi_{3} \sigma_{x}^{\prime}\right) & \left(\varphi_{4} \sigma_{y}^{\prime}\right) f_{22}\left(\varphi_{3} \sigma_{y}^{\prime}\right) & \mathbf{0} \\ \mathbf{0} & \mathbf{0} & \left(\varphi_{4} \tau_{x y}^{\prime}\right) f_{33} \varphi_{3} & \mathbf{0} & \mathbf{0} & \left(\varphi_{4} \tau_{x y}^{\prime}\right) \boldsymbol{f}_{33}\left(\varphi_{3} \tau_{x y}^{\prime}\right)\end{array}$

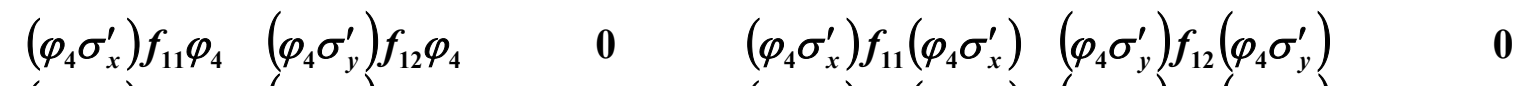

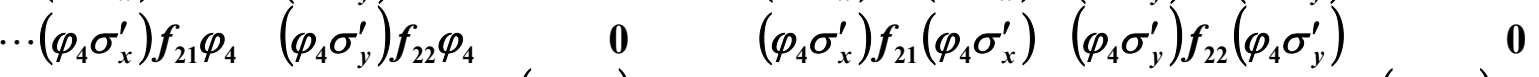

0

$0 \quad\left(\varphi_{4} \tau_{x y}^{\prime}\right) f_{33} \varphi_{4}$

0

$0 \quad\left(\varphi_{4} \tau_{x y}^{\prime}\right) f_{33}\left(\varphi_{4} \tau_{x y}^{\prime}\right)$

Onde $\boldsymbol{f}_{11}, \boldsymbol{f}_{12}, \boldsymbol{f}_{21}, \boldsymbol{f}_{22}$ e $\boldsymbol{f}_{33}$ são os termos da matriz de flexibilidade definida na eq.(2.6).

A matriz $\boldsymbol{A}_{\Omega e}$, dada pela eq.(4.12), terá uma dimensão:

- De (24 x 8), considerando-se somente os campos de tensão enriquecidos, como mostra a eq.(E.7). 
Linhas 1,2 e 3 da matriz $\boldsymbol{A}_{\Omega e}$ :

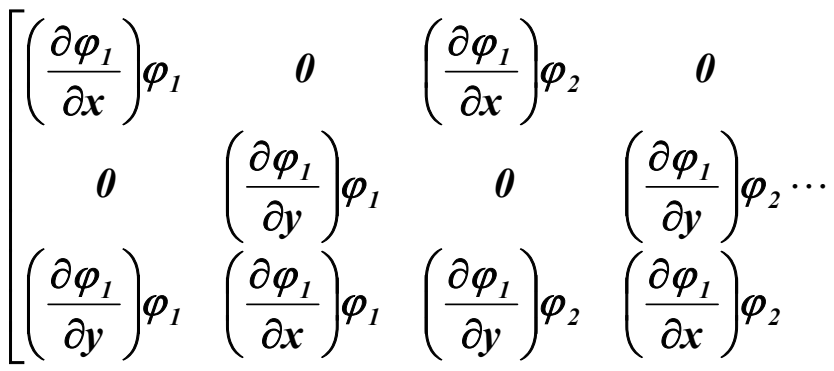

$$
\begin{aligned}
& \left(\frac{\partial \varphi_{1}}{\partial x}\right) \varphi_{3} \quad 0 \quad\left(\frac{\partial \varphi_{1}}{\partial x}\right) \varphi_{4} \quad \text { 0 }
\end{aligned}
$$

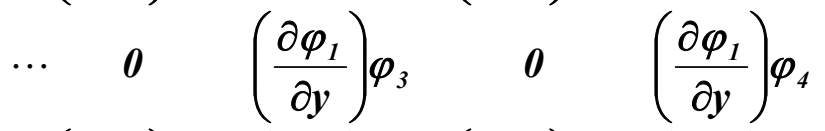

$$
\begin{aligned}
& \left.\left(\frac{\partial \varphi_{1}}{\partial y}\right) \varphi_{3} \quad\left(\frac{\partial \varphi_{1}}{\partial x}\right) \varphi_{3} \quad\left(\frac{\partial \varphi_{1}}{\partial y}\right) \varphi_{4} \quad\left(\frac{\partial \varphi_{1}}{\partial x}\right) \varphi_{4}\right]
\end{aligned}
$$

Linhas 4, 5 e 6 da matriz $\boldsymbol{A}_{\Omega e}$ :

$$
\begin{aligned}
& {\left[\begin{array}{cccc}
\left(\frac{\partial\left(\varphi_{1} \sigma_{x}^{\prime}\right)}{\partial x}\right) \varphi_{1} & \mathbf{0} & \left(\frac{\partial\left(\varphi_{1} \sigma_{x}^{\prime}\right)}{\partial x}\right) \varphi_{2} & \mathbf{0} \\
\mathbf{0} & \left(\frac{\partial\left(\varphi_{1} \sigma_{y}^{\prime}\right)}{\partial y}\right) \varphi_{1} & \mathbf{0} & \left(\frac{\partial\left(\varphi_{1} \sigma_{y}^{\prime}\right)}{\partial y}\right) \varphi_{2} \cdots \\
\left(\frac{\partial\left(\varphi_{1} \tau_{x y}^{\prime}\right)}{\partial y}\right) \varphi_{1} & \left(\frac{\partial\left(\varphi_{1} \tau_{x y}^{\prime}\right)}{\partial x}\right) \varphi_{1} & \left(\frac{\partial\left(\varphi_{1} \tau_{x y}^{\prime}\right)}{\partial y}\right) \varphi_{2} & \left(\frac{\partial\left(\varphi_{1} \tau_{x y}^{\prime}\right)}{\partial x}\right) \varphi_{2}
\end{array}\right.} \\
& \left(\frac{\partial\left(\varphi_{1} \sigma_{x}^{\prime}\right)}{\partial x}\right) \varphi_{3} \quad 0 \quad\left(\frac{\partial\left(\varphi_{1} \sigma_{x}^{\prime}\right)}{\partial x}\right) \varphi_{4} \quad \mathbf{0}
\end{aligned}
$$

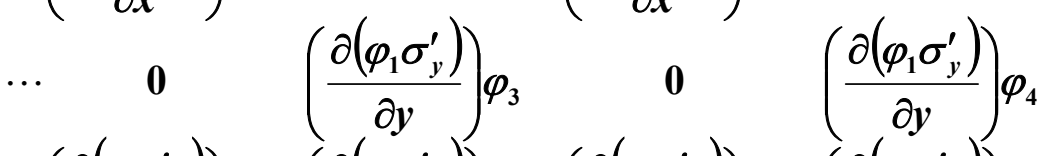

$$
\begin{aligned}
& \left.\left(\frac{\partial\left(\varphi_{1} \tau_{x y}^{\prime}\right)}{\partial y}\right) \varphi_{3} \quad\left(\frac{\partial\left(\varphi_{1} \tau_{x y}^{\prime}\right)}{\partial x}\right) \varphi_{3} \quad\left(\frac{\partial\left(\varphi_{1} \tau_{x y}^{\prime}\right)}{\partial y}\right) \varphi_{4} \quad\left(\frac{\partial\left(\varphi_{1} \tau_{x y}^{\prime}\right)}{\partial x}\right) \varphi_{4}\right]
\end{aligned}
$$


Linhas 7, 8 e 9 da matriz $\boldsymbol{A}_{\Omega e}$ :

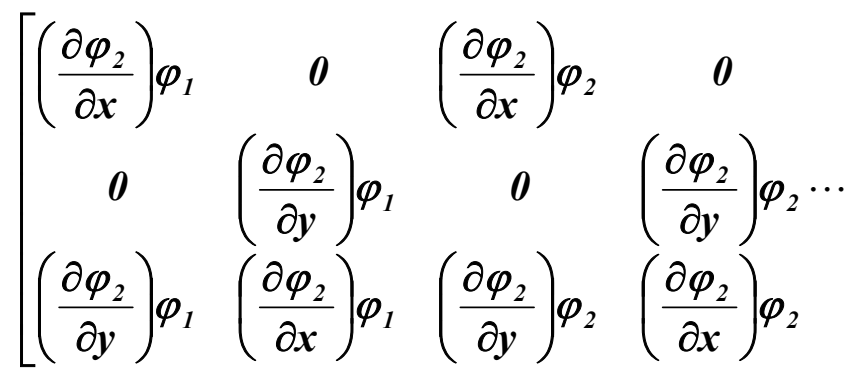

$$
\begin{aligned}
& \left(\frac{\partial \varphi_{2}}{\partial x}\right) \varphi_{3} \quad 0 \quad\left(\frac{\partial \varphi_{2}}{\partial x}\right) \varphi_{4} \quad \text { 0 }
\end{aligned}
$$

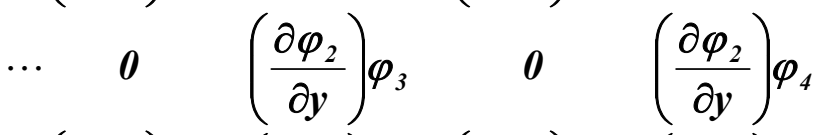

$$
\begin{aligned}
& \left.\left(\frac{\partial \varphi_{2}}{\partial y}\right) \varphi_{3} \quad\left(\frac{\partial \varphi_{2}}{\partial x}\right) \varphi_{3} \quad\left(\frac{\partial \varphi_{2}}{\partial y}\right) \varphi_{4} \quad\left(\frac{\partial \varphi_{2}}{\partial x}\right) \varphi_{4}\right]
\end{aligned}
$$

Linhas 10,11 e 12 da matriz $\boldsymbol{A}_{\Omega e}$ :

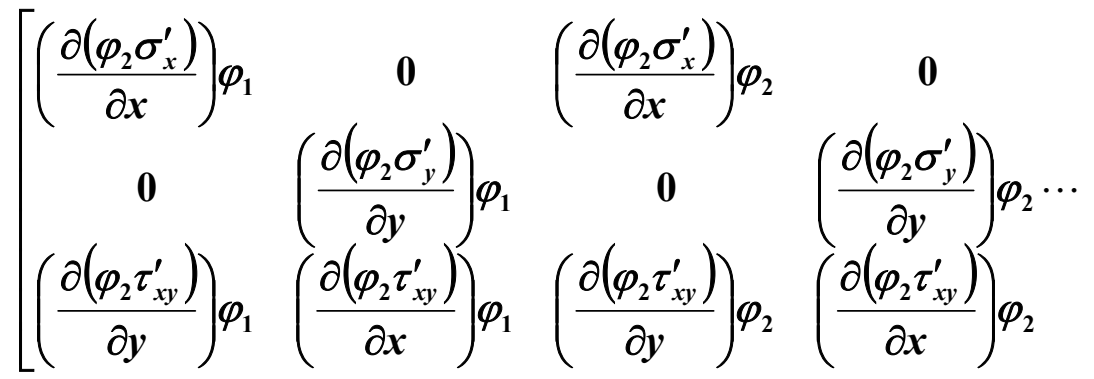

$$
\begin{aligned}
& \left(\frac{\partial\left(\varphi_{2} \sigma_{x}^{\prime}\right)}{\partial x}\right) \varphi_{3} \quad \mathbf{0} \quad\left(\frac{\partial\left(\varphi_{2} \sigma_{x}^{\prime}\right)}{\partial x}\right) \varphi_{4} \quad \mathbf{0}
\end{aligned}
$$

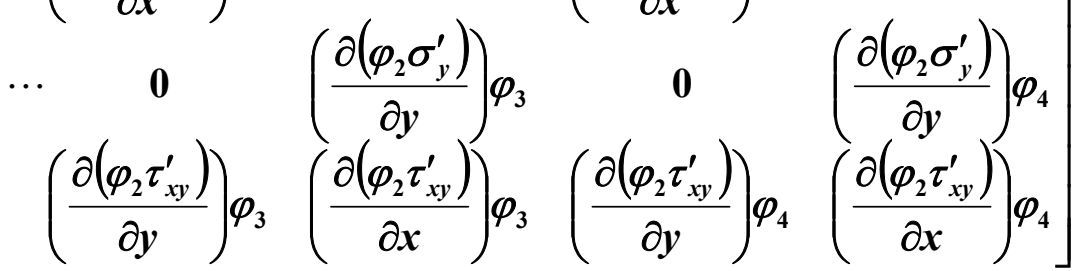

Linhas 13,14 e 15 da matriz $\boldsymbol{A}_{\Omega e}$ :

$$
\begin{aligned}
& {\left[\left(\frac{\partial \varphi_{3}}{\partial x}\right) \varphi_{1} \quad 0 \quad\left(\frac{\partial \varphi_{3}}{\partial x}\right) \varphi_{2} \quad 0\right.} \\
& \text { o } \quad\left(\frac{\partial \varphi_{3}}{\partial y}\right) \varphi_{1} \quad \text { o } \quad\left(\frac{\partial \varphi_{3}}{\partial y}\right) \varphi_{2} \cdots \\
& \left(\frac{\partial \varphi_{3}}{\partial y}\right) \varphi_{1} \quad\left(\frac{\partial \varphi_{3}}{\partial x}\right) \varphi_{1} \quad\left(\frac{\partial \varphi_{3}}{\partial y}\right) \varphi_{2} \quad\left(\frac{\partial \varphi_{3}}{\partial x}\right) \varphi_{2}
\end{aligned}
$$




$$
\begin{aligned}
& \left(\frac{\partial \varphi_{3}}{\partial x}\right) \varphi_{3} \quad 0 \quad\left(\frac{\partial \varphi_{3}}{\partial x}\right) \varphi_{4} \quad 0
\end{aligned}
$$

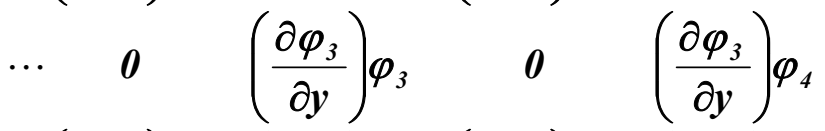

$$
\begin{aligned}
& \left(\frac{\partial \varphi_{3}}{\partial y}\right) \varphi_{3} \quad\left(\frac{\partial \varphi_{3}}{\partial x}\right) \varphi_{3} \quad\left(\frac{\partial \varphi_{3}}{\partial y}\right) \varphi_{4} \quad\left(\frac{\partial \varphi_{3}}{\partial x}\right) \varphi_{4}
\end{aligned}
$$

Linhas 16,17 e 18 da matriz $\boldsymbol{A}_{\Omega e}$ :

$$
\begin{aligned}
& {\left[\left(\frac{\partial\left(\varphi_{3} \sigma_{x}^{\prime}\right)}{\partial x}\right) \varphi_{1} \quad \text { 0 } \quad\left(\frac{\partial\left(\varphi_{3} \sigma_{x}^{\prime}\right)}{\partial x}\right) \varphi_{2} \quad \mathbf{0}\right.} \\
& \text { 0 } \quad\left(\frac{\partial\left(\varphi_{3} \sigma_{y}^{\prime}\right)}{\partial y}\right) \varphi_{1} \quad 0 \quad\left(\frac{\partial\left(\varphi_{3} \sigma_{y}^{\prime}\right)}{\partial y}\right) \varphi_{2} \cdots \\
& \left(\frac{\partial\left(\varphi_{3} \tau_{x y}^{\prime}\right)}{\partial y}\right) \varphi_{1} \quad\left(\frac{\partial\left(\varphi_{3} \tau_{x y}^{\prime}\right)}{\partial x}\right) \varphi_{1} \quad\left(\frac{\partial\left(\varphi_{3} \tau_{x y}^{\prime}\right)}{\partial y}\right) \varphi_{2} \quad\left(\frac{\partial\left(\varphi_{3} \tau_{x y}^{\prime}\right)}{\partial x}\right) \varphi_{2} \\
& \left(\frac{\partial\left(\varphi_{3} \sigma_{x}^{\prime}\right)}{\partial x}\right) \varphi_{3} \quad \mathbf{0} \quad\left(\frac{\partial\left(\varphi_{3} \sigma_{x}^{\prime}\right)}{\partial x}\right) \varphi_{4} \quad \mathbf{0}
\end{aligned}
$$

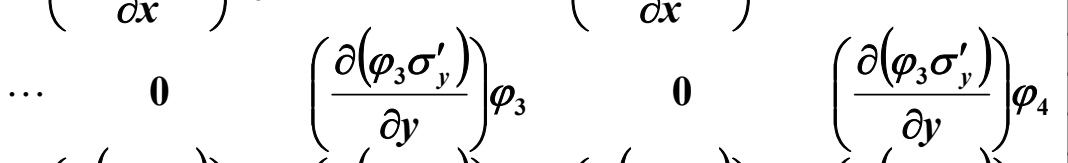

$$
\begin{aligned}
& \left.\left(\frac{\partial\left(\varphi_{3} \tau_{x y}^{\prime}\right)}{\partial y}\right) \varphi_{3} \quad\left(\frac{\partial\left(\varphi_{3} \tau_{x y}^{\prime}\right)}{\partial x}\right) \varphi_{3} \quad\left(\frac{\partial\left(\varphi_{3} \tau_{x y}^{\prime}\right)}{\partial y}\right) \varphi_{4} \quad\left(\frac{\partial\left(\varphi_{3} \tau_{x y}^{\prime}\right)}{\partial x}\right) \varphi_{4}\right]
\end{aligned}
$$

Linhas 19,20 e 21 da matriz $\boldsymbol{A}_{\boldsymbol{\Omega} e}$ :

$$
\begin{aligned}
& {\left[\left(\frac{\partial \varphi_{4}}{\partial x}\right) \varphi_{1} \quad 0 \quad\left(\frac{\partial \varphi_{4}}{\partial x}\right) \varphi_{2} \quad 0\right.} \\
& \text { 0 } \quad\left(\frac{\partial \varphi_{4}}{\partial y}\right) \varphi_{1} \quad \text { o } \quad\left(\frac{\partial \varphi_{4}}{\partial y}\right) \varphi_{2} \cdots \\
& \left(\frac{\partial \varphi_{4}}{\partial y}\right) \varphi_{1} \quad\left(\frac{\partial \varphi_{4}}{\partial x}\right) \varphi_{1} \quad\left(\frac{\partial \varphi_{4}}{\partial y}\right) \varphi_{2} \quad\left(\frac{\partial \varphi_{4}}{\partial x}\right) \varphi_{2}
\end{aligned}
$$

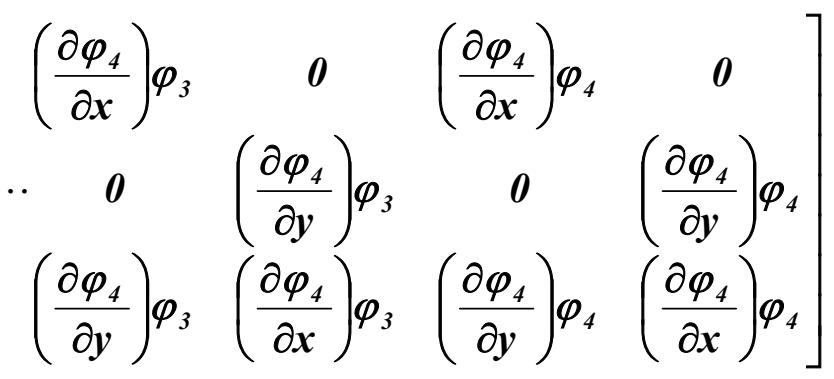


Linhas 22, 23 e 24 da matriz $\boldsymbol{A}_{\Omega e}$ :

$$
\begin{aligned}
& {\left[\begin{array}{cccc}
\left(\frac{\partial\left(\varphi_{4} \sigma_{x}^{\prime}\right)}{\partial x}\right) \varphi_{1} & \mathbf{0} & \left.\frac{\partial\left(\varphi_{4} \sigma_{x}^{\prime}\right)}{\partial x}\right) \varphi_{2} & \mathbf{0} \\
\mathbf{0} & \left(\frac{\partial\left(\varphi_{4} \sigma_{y}^{\prime}\right)}{\partial y}\right) \varphi_{1} & \mathbf{0} & \left.\frac{\partial\left(\varphi_{4} \sigma_{y}^{\prime}\right)}{\partial y}\right) \varphi_{2} \cdots \\
\left(\frac{\partial\left(\varphi_{4} \tau_{x y}^{\prime}\right)}{\partial y}\right) \varphi_{1} & \left(\frac{\partial\left(\varphi_{4} \tau_{x y}^{\prime}\right)}{\partial x}\right) \varphi_{1} & \left(\frac{\partial\left(\varphi_{4} \tau_{x y}^{\prime}\right)}{\partial y}\right) \varphi_{2} & \left(\frac{\partial\left(\varphi_{4} \tau_{x y}^{\prime}\right)}{\partial x}\right) \varphi_{2}
\end{array}\right.} \\
& \left.\begin{array}{cccc}
\left(\frac{\partial\left(\varphi_{4} \sigma_{x}^{\prime}\right)}{\partial x}\right) \varphi_{3} & \mathbf{0} & \left(\frac{\partial\left(\varphi_{4} \sigma_{x}^{\prime}\right)}{\partial x}\right) \varphi_{4} & \mathbf{0} \\
0 & \left(\frac{\partial\left(\varphi_{4} \sigma_{y}^{\prime}\right)}{\partial y}\right) \varphi_{3} & \mathbf{0} & \left.\frac{\partial\left(\varphi_{4} \sigma_{y}^{\prime}\right)}{\partial y}\right) \varphi_{4} \\
\left(\frac{\partial\left(\varphi_{4} \tau_{x y}^{\prime}\right)}{\partial y}\right) \varphi_{3} & \left(\frac{\partial\left(\varphi_{4} \tau_{x y}^{\prime}\right)}{\partial x}\right) \varphi_{3} & \left(\frac{\partial\left(\varphi_{4} \tau_{x y}^{\prime}\right)}{\partial y}\right) \varphi_{4} & \left(\frac{\partial\left(\varphi_{4} \tau_{x y}^{\prime}\right)}{\partial x}\right) \varphi_{4}
\end{array}\right]
\end{aligned}
$$

- De (24 x 16), considerando-se os campos de tensão e deslocamentos enriquecidos, como mostra a eq.(E.8).

Linhas 1,2 e 3 da matriz $\boldsymbol{A}_{\Omega e}$ :

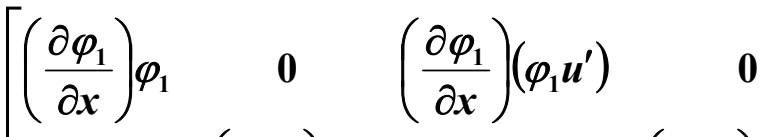

$$
\begin{aligned}
& \text { 0 } \quad\left(\frac{\partial \varphi_{1}}{\partial y}\right) \varphi_{1} \quad 0 \quad\left(\frac{\partial \varphi_{1}}{\partial y}\right)\left(\varphi_{1} v^{\prime}\right) \ldots \\
& {\left[\left(\frac{\partial \varphi_{1}}{\partial y}\right) \varphi_{1} \quad\left(\frac{\partial \varphi_{1}}{\partial x}\right) \varphi_{1} \quad\left(\frac{\partial \varphi_{1}}{\partial y}\right)\left(\varphi_{1} u^{\prime}\right) \quad\left(\frac{\partial \varphi_{1}}{\partial x}\right)\left(\varphi_{1} v^{\prime}\right)\right.}
\end{aligned}
$$

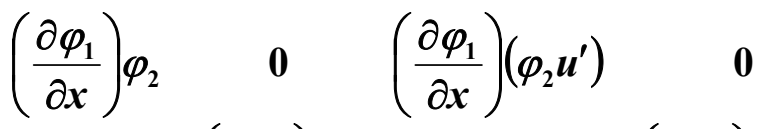

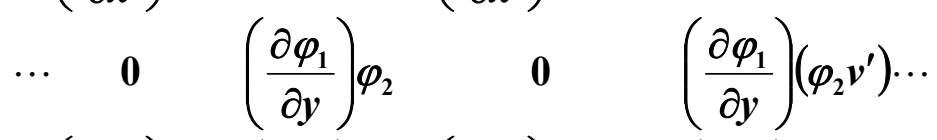

$$
\begin{aligned}
& \left(\frac{\partial \varphi_{1}}{\partial y}\right) \varphi_{2} \quad\left(\frac{\partial \varphi_{1}}{\partial x}\right) \varphi_{2} \quad\left(\frac{\partial \varphi_{1}}{\partial y}\right)\left(\varphi_{2} u^{\prime}\right)\left(\frac{\partial \varphi_{1}}{\partial x}\right)\left(\varphi_{2} v^{\prime}\right)
\end{aligned}
$$




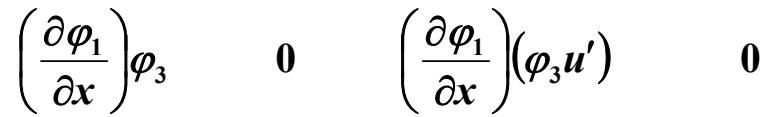

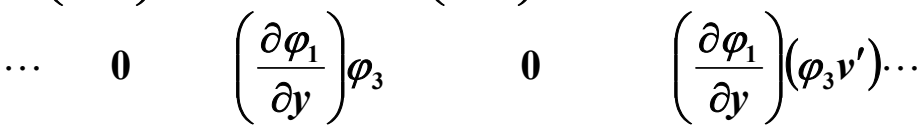

$$
\begin{aligned}
& \left(\frac{\partial \varphi_{1}}{\partial y}\right) \varphi_{3} \quad\left(\frac{\partial \varphi_{1}}{\partial x}\right) \varphi_{3} \quad\left(\frac{\partial \varphi_{1}}{\partial y}\right)\left(\varphi_{3} u^{\prime}\right)\left(\frac{\partial \varphi_{1}}{\partial x}\right)\left(\varphi_{3} v^{\prime}\right)
\end{aligned}
$$

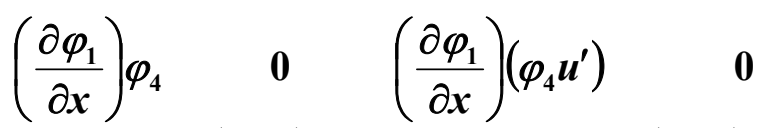

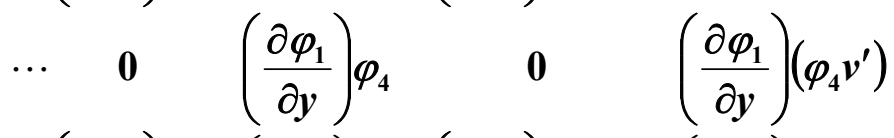

$$
\begin{aligned}
& \left(\frac{\partial \varphi_{1}}{\partial y}\right) \varphi_{4} \quad\left(\frac{\partial \varphi_{1}}{\partial x}\right) \varphi_{4} \quad\left(\frac{\partial \varphi_{1}}{\partial y}\right)\left(\varphi_{4} u^{\prime}\right)\left(\frac{\partial \varphi_{1}}{\partial x}\right)\left(\varphi_{4} v^{\prime}\right)
\end{aligned}
$$

Linhas 4, 5 e 6 da matriz $\boldsymbol{A}_{\Omega_{e}}$ :

$$
\begin{aligned}
& {\left[\begin{array}{cccc}
\left(\frac{\partial\left(\varphi_{1} \sigma_{x}^{\prime}\right)}{\partial x}\right) \varphi_{1} & \mathbf{0} & \left(\frac{\partial\left(\varphi_{1} \sigma_{x}^{\prime}\right)}{\partial x}\right)\left(\varphi_{1} \boldsymbol{u}^{\prime}\right) & \mathbf{0} \\
\mathbf{0} & \left(\frac{\partial\left(\varphi_{1} \sigma_{y}^{\prime}\right)}{\partial y}\right) \varphi_{1} & \mathbf{0} & \left(\frac{\partial\left(\varphi_{1} \sigma_{y}^{\prime}\right)}{\partial y}\right)\left(\varphi_{1} v^{\prime}\right) \ldots \\
\left(\frac{\partial\left(\varphi_{1} \tau_{x y}^{\prime}\right)}{\partial y}\right) \varphi_{1} & \left(\frac{\partial\left(\varphi_{1} \tau_{x y}^{\prime}\right)}{\partial x}\right) \varphi_{1} & \left(\frac{\partial\left(\varphi_{1} \tau_{x y}^{\prime}\right)}{\partial y}\right)\left(\varphi_{1} u^{\prime}\right) & \left(\frac{\partial\left(\varphi_{1} \tau_{x y}^{\prime}\right)}{\partial x}\right)\left(\varphi_{1} v^{\prime}\right)
\end{array}\right.} \\
& \left(\frac{\partial\left(\varphi_{1} \sigma_{x}^{\prime}\right)}{\partial x}\right) \varphi_{2} \quad \text { 0 } \quad\left(\frac{\partial\left(\varphi_{1} \sigma_{x}^{\prime}\right)}{\partial x}\right)\left(\varphi_{2} u^{\prime}\right) \quad \text { 0 }
\end{aligned}
$$

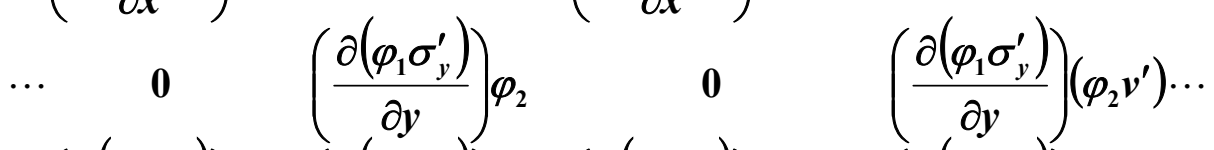

$$
\begin{aligned}
& \left(\frac{\partial\left(\varphi_{1} \tau_{x y}^{\prime}\right)}{\partial y}\right) \varphi_{2}\left(\frac{\partial\left(\varphi_{1} \tau_{x y}^{\prime}\right)}{\partial x}\right) \varphi_{2}\left(\frac{\partial\left(\varphi_{1} \tau_{x y}^{\prime}\right)}{\partial y}\right)\left(\varphi_{2} u^{\prime}\right)\left(\frac{\partial\left(\varphi_{1} \tau_{x y}^{\prime}\right)}{\partial x}\right)\left(\varphi_{2} v^{\prime}\right) \\
& \left(\frac{\partial\left(\varphi_{1} \sigma_{x}^{\prime}\right)}{\partial x}\right) \varphi_{3} \quad \mathbf{0} \quad\left(\frac{\partial\left(\varphi_{1} \sigma_{x}^{\prime}\right)}{\partial x}\right)\left(\varphi_{3} \boldsymbol{u}^{\prime}\right) \quad \mathbf{0}
\end{aligned}
$$

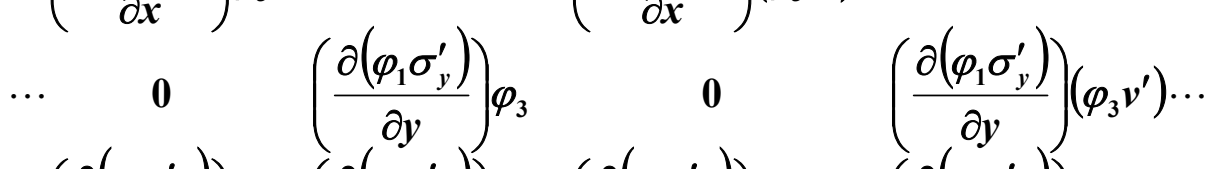

$$
\begin{aligned}
& \left(\frac{\partial\left(\varphi_{1} \tau_{x y}^{\prime}\right)}{\partial y}\right) \varphi_{3} \quad\left(\frac{\partial\left(\varphi_{1} \tau_{x y}^{\prime}\right)}{\partial x}\right) \varphi_{3} \quad\left(\frac{\partial\left(\varphi_{1} \tau_{x y}^{\prime}\right)}{\partial y}\right)\left(\varphi_{3} u^{\prime}\right)\left(\frac{\partial\left(\varphi_{1} \tau_{x y}^{\prime}\right)}{\partial x}\right)\left(\varphi_{3} v^{\prime}\right)
\end{aligned}
$$




$$
\begin{aligned}
& \left(\frac{\partial\left(\varphi_{1} \sigma_{x}^{\prime}\right)}{\partial x}\right) \varphi_{4} \quad \text { 0 } \quad\left(\frac{\partial\left(\varphi_{1} \sigma_{x}^{\prime}\right)}{\partial \boldsymbol{x}}\right)\left(\varphi_{4} \boldsymbol{u}^{\prime}\right) \quad \text { 0 }
\end{aligned}
$$

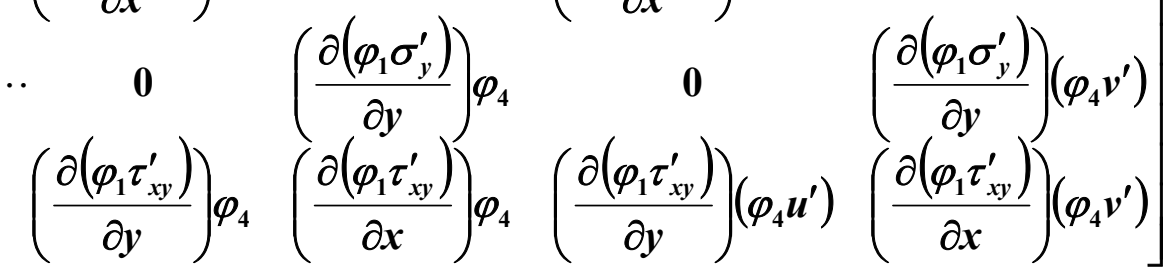

Linhas 7, 8 e 9 da matriz $\boldsymbol{A}_{\Omega e}$ :

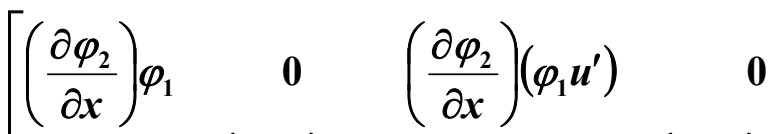

$$
\begin{aligned}
& \text { 0 }\left(\frac{\partial \varphi_{2}}{\partial y}\right) \varphi_{1} \quad 0 \quad\left(\frac{\partial \varphi_{2}}{\partial y}\right)\left(\varphi_{1} v^{\prime}\right) \cdots \\
& {\left[\left(\frac{\partial \varphi_{2}}{\partial y}\right) \varphi_{1}\left(\frac{\partial \varphi_{2}}{\partial x}\right) \varphi_{1} \quad\left(\frac{\partial \varphi_{2}}{\partial y}\right)\left(\varphi_{1} u^{\prime}\right)\left(\frac{\partial \varphi_{2}}{\partial x}\right)\left(\varphi_{1} v^{\prime}\right)\right.} \\
& \left(\frac{\partial \varphi_{2}}{\partial \boldsymbol{x}}\right) \varphi_{2} \quad \text { 0 } \quad\left(\frac{\partial \varphi_{2}}{\partial \boldsymbol{x}}\right)\left(\varphi_{2} \boldsymbol{u}^{\prime}\right) \quad \text { 0 }
\end{aligned}
$$

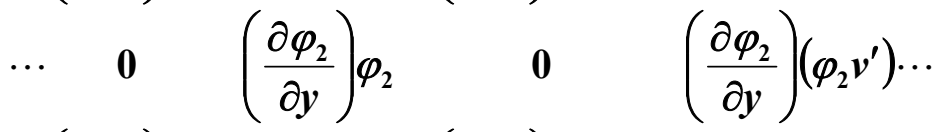

$$
\begin{aligned}
& \left(\frac{\partial \varphi_{2}}{\partial y}\right) \varphi_{2}\left(\frac{\partial \varphi_{2}}{\partial x}\right) \varphi_{2}\left(\frac{\partial \varphi_{2}}{\partial y}\right)\left(\varphi_{2} u^{\prime}\right)\left(\frac{\partial \varphi_{2}}{\partial x}\right)\left(\varphi_{2} v^{\prime}\right)
\end{aligned}
$$

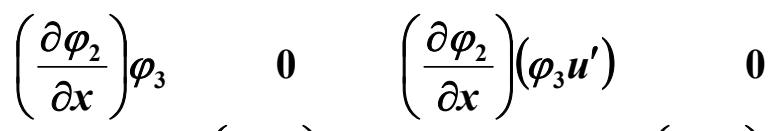

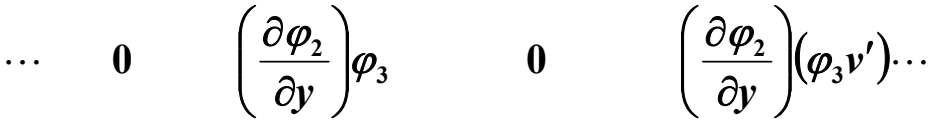

$$
\begin{aligned}
& \left(\frac{\partial \varphi_{2}}{\partial y}\right) \varphi_{3}\left(\frac{\partial \varphi_{2}}{\partial x}\right) \varphi_{3} \quad\left(\frac{\partial \varphi_{2}}{\partial y}\right)\left(\varphi_{3} u^{\prime}\right)\left(\frac{\partial \varphi_{2}}{\partial x}\right)\left(\varphi_{3} v^{\prime}\right)
\end{aligned}
$$

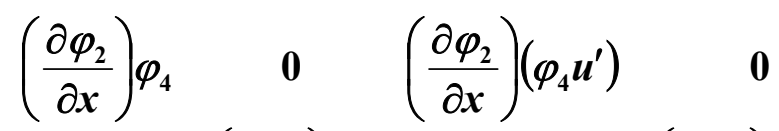

$$
\begin{aligned}
& \begin{array}{ccccc}
\cdots & 0 & \left(\frac{\partial \varphi_{2}}{\partial y}\right) \varphi_{4} & 0 & \left(\frac{\partial \varphi_{2}}{\partial y}\right)\left(\varphi_{4} v^{\prime}\right)
\end{array} \\
& \left.\left(\frac{\partial \varphi_{2}}{\partial y}\right) \varphi_{4} \quad\left(\frac{\partial \varphi_{2}}{\partial x}\right) \varphi_{4} \quad\left(\frac{\partial \varphi_{2}}{\partial y}\right)\left(\varphi_{4} u^{\prime}\right)\left(\frac{\partial \varphi_{2}}{\partial x}\right)\left(\varphi_{4} v^{\prime}\right)\right]
\end{aligned}
$$


Linhas 10,11 e 12 da matriz $\boldsymbol{A}_{\Omega e}$ :

$$
\begin{aligned}
& {\left[\begin{array}{cccc}
\left(\frac{\partial\left(\varphi_{2} \sigma_{x}^{\prime}\right)}{\partial x}\right) \varphi_{1} & \mathbf{0} & \left(\frac{\partial\left(\varphi_{2} \sigma_{x}^{\prime}\right)}{\partial x}\right)\left(\varphi_{1} u^{\prime}\right) & 0 \\
\mathbf{0} & \left(\frac{\partial\left(\varphi_{2} \sigma_{y}^{\prime}\right)}{\partial y}\right) \varphi_{1} & 0 & \left.\frac{\partial\left(\varphi_{2} \sigma_{y}^{\prime}\right)}{\partial y}\right)\left(\varphi_{1} v^{\prime}\right) \ldots \\
\left(\frac{\partial\left(\varphi_{2} \tau_{x y}^{\prime}\right)}{\partial y}\right) \varphi_{1} & \left(\frac{\partial\left(\varphi_{2} \tau_{x y}^{\prime}\right)}{\partial x}\right) \varphi_{1} & \left(\frac{\partial\left(\varphi_{2} \tau_{x y}^{\prime}\right)}{\partial y}\right)\left(\varphi_{1} u^{\prime}\right) & \left(\frac{\partial\left(\varphi_{2} \tau_{x y}^{\prime}\right)}{\partial x}\right)\left(\varphi_{1} v^{\prime}\right)
\end{array}\right.} \\
& \left(\frac{\partial\left(\varphi_{2} \sigma_{x}^{\prime}\right)}{\partial \boldsymbol{x}}\right) \varphi_{2} \quad \mathbf{0} \quad\left(\frac{\partial\left(\varphi_{2} \sigma_{x}^{\prime}\right)}{\partial \boldsymbol{x}}\right)\left(\varphi_{2} \boldsymbol{u}^{\prime}\right) \quad \mathbf{0}
\end{aligned}
$$

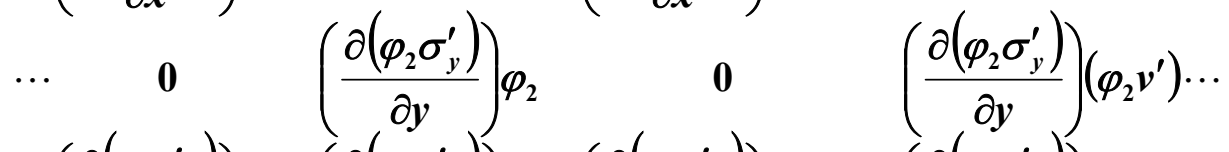

$$
\begin{aligned}
& \left(\frac{\partial\left(\varphi_{2} \tau_{x y}^{\prime}\right)}{\partial y}\right) \varphi_{2}\left(\frac{\partial\left(\varphi_{2} \tau_{x y}^{\prime}\right)}{\partial x}\right) \varphi_{2}\left(\frac{\partial\left(\varphi_{2} \tau_{x y}^{\prime}\right)}{\partial y}\right)\left(\varphi_{2} u^{\prime}\right)\left(\frac{\partial\left(\varphi_{2} \tau_{x y}^{\prime}\right)}{\partial x}\right)\left(\varphi_{2} v^{\prime}\right) \\
& \left(\frac{\partial\left(\varphi_{2} \sigma_{x}^{\prime}\right)}{\partial x}\right) \varphi_{3} \quad \mathbf{0} \quad\left(\frac{\partial\left(\varphi_{2} \sigma_{x}^{\prime}\right)}{\partial x}\right)\left(\varphi_{3} u^{\prime}\right) \quad \text { 0 }
\end{aligned}
$$

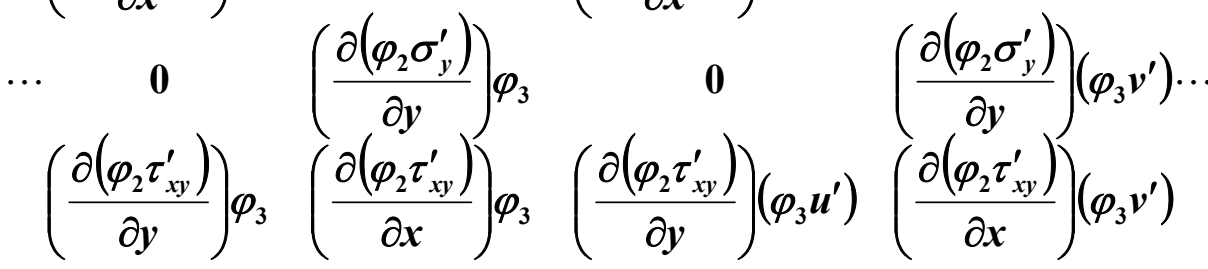

$$
\begin{aligned}
& \left(\frac{\partial\left(\varphi_{2} \sigma_{x}^{\prime}\right)}{\partial x}\right) \varphi_{4} \quad 0 \quad\left(\frac{\partial\left(\varphi_{2} \sigma_{x}^{\prime}\right)}{\partial x}\right)\left(\varphi_{4} u^{\prime}\right) \quad \text { 0 }
\end{aligned}
$$

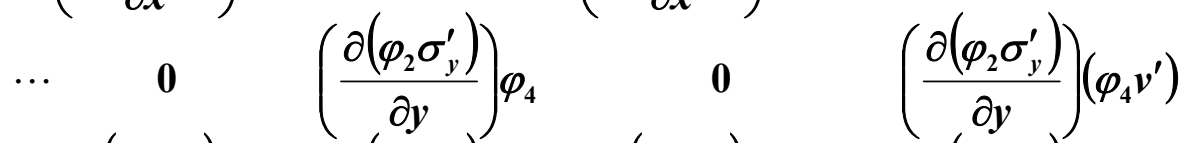

$$
\begin{aligned}
& \left.\left(\frac{\partial\left(\varphi_{2} \tau_{x y}^{\prime}\right)}{\partial y}\right) \varphi_{4}\left(\frac{\partial\left(\varphi_{2} \tau_{x y}^{\prime}\right)}{\partial x}\right) \varphi_{4}\left(\frac{\partial\left(\varphi_{2} \tau_{x y}^{\prime}\right)}{\partial y}\right)\left(\varphi_{4} u^{\prime}\right)\left(\frac{\partial\left(\varphi_{2} \tau_{x y}^{\prime}\right)}{\partial x}\right)\left(\varphi_{4} v^{\prime}\right)\right]
\end{aligned}
$$

Linhas 13,14 e 15 da matriz $A_{\Omega e}$ :

$$
\left[\begin{array}{cccc}
\left(\frac{\partial \varphi_{3}}{\partial x}\right) \varphi_{1} & \mathbf{0} & \left(\frac{\partial \varphi_{3}}{\partial x}\right)\left(\varphi_{1} u^{\prime}\right) & 0 \\
0 & \left(\frac{\partial \varphi_{3}}{\partial y}\right) \varphi_{1} & 0 & \left(\frac{\partial \varphi_{3}}{\partial y}\right)\left(\varphi_{1} v^{\prime}\right) \ldots \\
\left(\frac{\partial \varphi_{3}}{\partial y}\right) \varphi_{1} & \left(\frac{\partial \varphi_{3}}{\partial x}\right) \varphi_{1} & \left(\frac{\partial \varphi_{3}}{\partial y}\right)\left(\varphi_{1} u^{\prime}\right) & \left(\frac{\partial \varphi_{3}}{\partial x}\right)\left(\varphi_{1} v^{\prime}\right)
\end{array}\right.
$$




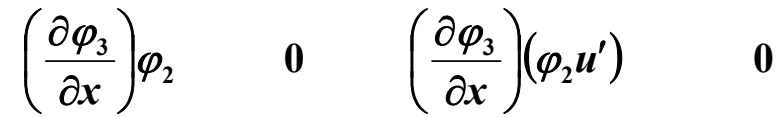

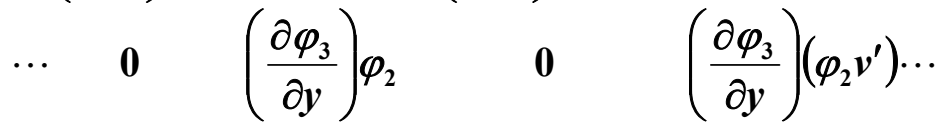

$$
\begin{aligned}
& \left(\frac{\partial \varphi_{3}}{\partial y}\right) \varphi_{2} \quad\left(\frac{\partial \varphi_{3}}{\partial x}\right) \varphi_{2} \quad\left(\frac{\partial \varphi_{3}}{\partial y}\right)\left(\varphi_{2} u^{\prime}\right)\left(\frac{\partial \varphi_{3}}{\partial x}\right)\left(\varphi_{2} v^{\prime}\right) \\
& \left(\frac{\partial \varphi_{3}}{\partial x}\right) \varphi_{3} \quad \text { o } \quad\left(\frac{\partial \varphi_{3}}{\partial x}\right)\left(\varphi_{3} u^{\prime}\right) \quad \text { 0 }
\end{aligned}
$$

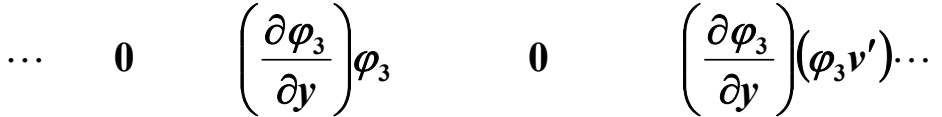

$$
\begin{aligned}
& \left(\frac{\partial \varphi_{3}}{\partial y}\right) \varphi_{3} \quad\left(\frac{\partial \varphi_{3}}{\partial x}\right) \varphi_{3} \quad\left(\frac{\partial \varphi_{3}}{\partial y}\right)\left(\varphi_{3} u^{\prime}\right)\left(\frac{\partial \varphi_{3}}{\partial x}\right)\left(\varphi_{3} v^{\prime}\right)
\end{aligned}
$$

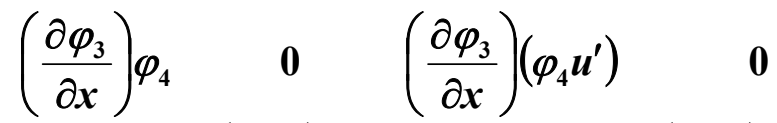

$$
\begin{aligned}
& \begin{array}{lllll}
\cdots & 0 & \left(\frac{\partial \varphi_{3}}{\partial y}\right) \varphi_{4} & 0 & \left(\frac{\partial \varphi_{3}}{\partial y}\right)\left(\varphi_{4} v^{\prime}\right)
\end{array} \\
& \left.\left(\frac{\partial \varphi_{3}}{\partial y}\right) \varphi_{4} \quad\left(\frac{\partial \varphi_{3}}{\partial x}\right) \varphi_{4} \quad\left(\frac{\partial \varphi_{3}}{\partial y}\right)\left(\varphi_{4} u^{\prime}\right) \quad\left(\frac{\partial \varphi_{3}}{\partial x}\right)\left(\varphi_{4} v^{\prime}\right)\right]
\end{aligned}
$$

Linhas 16,17 e 18 da matriz $\boldsymbol{A}_{\Omega e}$ :

$$
\begin{aligned}
& {\left[\left(\frac{\partial\left(\varphi_{3} \sigma_{x}^{\prime}\right)}{\partial x}\right) \varphi_{1} \quad \mathbf{0} \quad\left(\frac{\partial\left(\varphi_{3} \sigma_{x}^{\prime}\right)}{\partial \boldsymbol{x}}\right)\left(\varphi_{1} \boldsymbol{u}^{\prime}\right) \quad \mathbf{0}\right.} \\
& \text { 0 } \quad\left(\frac{\partial\left(\varphi_{3} \sigma_{y}^{\prime}\right)}{\partial y}\right) \varphi_{1} \quad 0 \quad\left(\frac{\partial\left(\varphi_{3} \sigma_{y}^{\prime}\right)}{\partial y}\right)\left(\varphi_{1} v^{\prime}\right) \ldots \\
& \left(\frac{\partial\left(\varphi_{3} \tau_{x y}^{\prime}\right)}{\partial y}\right) \varphi_{1}\left(\frac{\partial\left(\varphi_{3} \tau_{x y}^{\prime}\right)}{\partial x}\right) \varphi_{1} \quad\left(\frac{\partial\left(\varphi_{3} \tau_{x y}^{\prime}\right)}{\partial y}\right)\left(\varphi_{1} u^{\prime}\right)\left(\frac{\partial\left(\varphi_{3} \tau_{x y}^{\prime}\right)}{\partial x}\right)\left(\varphi_{1} v^{\prime}\right) \\
& \left(\frac{\partial\left(\varphi_{3} \sigma_{x}^{\prime}\right)}{\partial \boldsymbol{x}}\right) \varphi_{2} \quad \text { 0 } \quad\left(\frac{\partial\left(\varphi_{3} \sigma_{x}^{\prime}\right)}{\partial \boldsymbol{x}}\right)\left(\varphi_{2} \boldsymbol{u}^{\prime}\right) \quad \mathbf{0}
\end{aligned}
$$

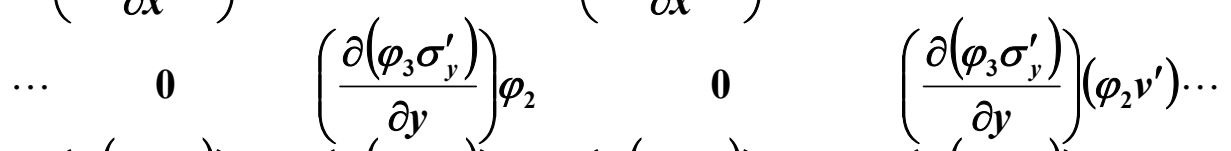

$$
\begin{aligned}
& \left(\frac{\partial\left(\varphi_{3} \tau_{x y}^{\prime}\right)}{\partial y}\right) \varphi_{2}\left(\frac{\partial\left(\varphi_{3} \tau_{x y}^{\prime}\right)}{\partial x}\right) \varphi_{2} \quad\left(\frac{\partial\left(\varphi_{3} \tau_{x y}^{\prime}\right)}{\partial y}\right)\left(\varphi_{2} u^{\prime}\right)\left(\frac{\partial\left(\varphi_{3} \tau_{x y}^{\prime}\right)}{\partial x}\right)\left(\varphi_{2} v^{\prime}\right)
\end{aligned}
$$




$$
\begin{aligned}
& \left(\frac{\partial\left(\varphi_{3} \sigma_{x}^{\prime}\right)}{\partial x}\right) \varphi_{3} \quad \text { 0 } \quad\left(\frac{\partial\left(\varphi_{3} \sigma_{x}^{\prime}\right)}{\partial x}\right)\left(\varphi_{3} u^{\prime}\right) \quad \text { 0 }
\end{aligned}
$$

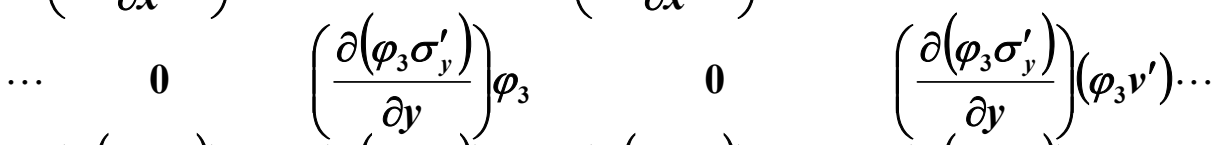

$$
\begin{aligned}
& \left(\frac{\partial\left(\varphi_{3} \tau_{x y}^{\prime}\right)}{\partial y}\right) \varphi_{3} \quad\left(\frac{\partial\left(\varphi_{3} \tau_{x y}^{\prime}\right)}{\partial x}\right) \varphi_{3} \quad\left(\frac{\partial\left(\varphi_{3} \tau_{x y}^{\prime}\right)}{\partial y}\right)\left(\varphi_{3} u^{\prime}\right)\left(\frac{\partial\left(\varphi_{3} \tau_{x y}^{\prime}\right)}{\partial x}\right)\left(\varphi_{3} v^{\prime}\right) \\
& \left(\frac{\partial\left(\varphi_{3} \sigma_{x}^{\prime}\right)}{\partial x}\right) \varphi_{4} \quad 0 \quad\left(\frac{\partial\left(\varphi_{3} \sigma_{x}^{\prime}\right)}{\partial x}\right)\left(\varphi_{4} u^{\prime}\right)
\end{aligned}
$$

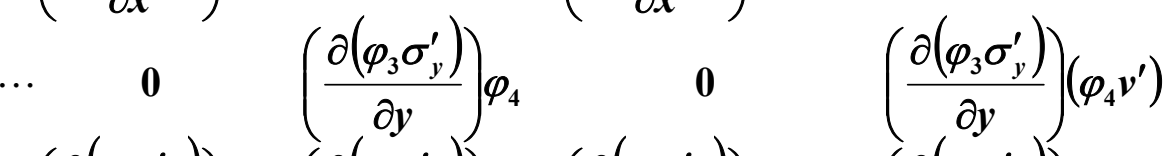

$$
\begin{aligned}
& \left(\frac{\partial\left(\varphi_{3} \tau_{x y}^{\prime}\right)}{\partial y}\right) \varphi_{4}\left(\frac{\partial\left(\varphi_{3} \tau_{x y}^{\prime}\right)}{\partial x}\right) \varphi_{4}\left(\frac{\partial\left(\varphi_{3} \tau_{x y}^{\prime}\right)}{\partial y}\right)\left(\varphi_{4} u^{\prime}\right)\left(\frac{\partial\left(\varphi_{3} \tau_{x y}^{\prime}\right)}{\partial x}\right)\left(\varphi_{4} v^{\prime}\right)
\end{aligned}
$$

Linhas 19,20 e 21 da matriz $\boldsymbol{A}_{\boldsymbol{\Omega} e}$ :

$$
\begin{aligned}
& {\left[\left(\begin{array}{lll}
\left.\frac{\partial \varphi_{4}}{\partial x}\right) \varphi_{1} & \mathbf{0} & \left(\frac{\partial \varphi_{4}}{\partial x}\right)\left(\varphi_{1} u^{\prime}\right) \quad \text { 0 }
\end{array}\right.\right.} \\
& 0 \quad\left(\frac{\partial \varphi_{4}}{\partial y}\right) \varphi_{1} \quad 0 \quad\left(\frac{\partial \varphi_{4}}{\partial y}\right)\left(\varphi_{1} v^{\prime}\right) \ldots \\
& \left(\frac{\partial \varphi_{4}}{\partial y}\right) \varphi_{1} \quad\left(\frac{\partial \varphi_{4}}{\partial x}\right) \varphi_{1} \quad\left(\frac{\partial \varphi_{4}}{\partial y}\right)\left(\varphi_{1} v^{\prime}\right) \quad\left(\frac{\partial \varphi_{4}}{\partial x}\right)\left(\varphi_{1} v^{\prime}\right)
\end{aligned}
$$

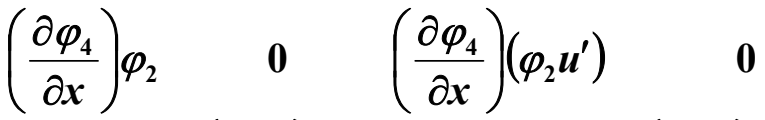

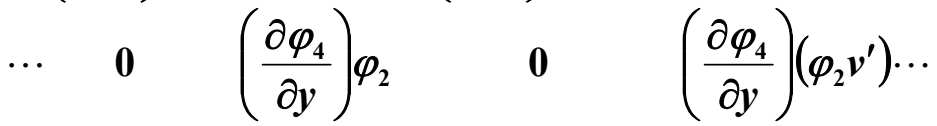

$$
\begin{aligned}
& \left(\frac{\partial \varphi_{4}}{\partial y}\right) \varphi_{2} \quad\left(\frac{\partial \varphi_{4}}{\partial x}\right) \varphi_{2} \quad\left(\frac{\partial \varphi_{4}}{\partial y}\right)\left(\varphi_{2} u^{\prime}\right)\left(\frac{\partial \varphi_{4}}{\partial x}\right)\left(\varphi_{2} v^{\prime}\right) \\
& \left(\frac{\partial \varphi_{4}}{\partial x}\right) \varphi_{3} \quad \text { 0 } \quad\left(\frac{\partial \varphi_{4}}{\partial x}\right)\left(\varphi_{3} \boldsymbol{u}^{\prime}\right) \quad \text { 0 }
\end{aligned}
$$

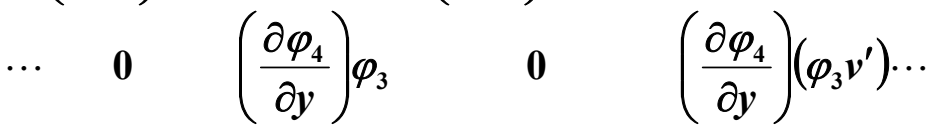

$$
\begin{aligned}
& \left(\frac{\partial \varphi_{4}}{\partial y}\right) \varphi_{3} \quad\left(\frac{\partial \varphi_{4}}{\partial x}\right) \varphi_{3} \quad\left(\frac{\partial \varphi_{4}}{\partial y}\right)\left(\varphi_{3} u^{\prime}\right) \quad\left(\frac{\partial \varphi_{4}}{\partial x}\right)\left(\varphi_{3} v^{\prime}\right)
\end{aligned}
$$




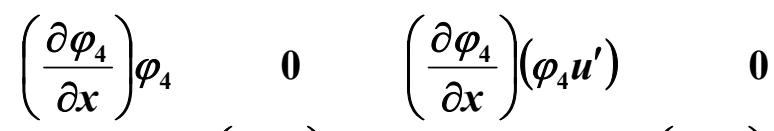

$$
\begin{aligned}
& \begin{array}{ccccc}
\cdots & 0 & \left(\frac{\partial \varphi_{4}}{\partial y}\right) \varphi_{4} & 0 & \left(\frac{\partial \varphi_{4}}{\partial y}\right)\left(\varphi_{4} v^{\prime}\right)
\end{array} \\
& \left.\left(\frac{\partial \varphi_{4}}{\partial y}\right) \varphi_{4} \quad\left(\frac{\partial \varphi_{4}}{\partial x}\right) \varphi_{4} \quad\left(\frac{\partial \varphi_{4}}{\partial y}\right)\left(\varphi_{4} u^{\prime}\right) \quad\left(\frac{\partial \varphi_{4}}{\partial x}\right)\left(\varphi_{4} v^{\prime}\right)\right]
\end{aligned}
$$

Linhas 22,23 e 24 da matriz $\boldsymbol{A}_{\Omega e}$ :

$$
\begin{aligned}
& {\left[\begin{array}{cccc}
\left(\frac{\partial\left(\varphi_{4} \sigma_{x}^{\prime}\right)}{\partial x}\right) \varphi_{1} & \mathbf{0} & \left(\frac{\partial\left(\varphi_{4} \sigma_{x}^{\prime}\right)}{\partial x}\right)\left(\varphi_{1} u^{\prime}\right) & 0 \\
\mathbf{0} & \left(\frac{\partial\left(\varphi_{4} \sigma_{y}\right)}{\partial y}\right) \varphi_{1} & 0 & \left.\frac{\partial\left(\varphi_{4} \sigma_{y}^{\prime}\right)}{\partial y}\right)\left(\varphi_{1} v^{\prime}\right) \ldots \\
\left(\frac{\partial\left(\varphi_{4} \tau_{x y}^{\prime}\right)}{\partial y}\right) \varphi_{1} & \left(\frac{\partial\left(\varphi_{4} \tau_{x y}^{\prime}\right)}{\partial x}\right) \varphi_{1} & \left(\frac{\partial\left(\varphi_{4} \tau_{x y}^{\prime}\right)}{\partial y}\right)\left(\varphi_{1} u^{\prime}\right) & \left(\frac{\partial\left(\varphi_{4} \tau_{x y}^{\prime}\right)}{\partial x}\right)\left(\varphi_{1} v^{\prime}\right)
\end{array}\right.} \\
& \left(\frac{\partial\left(\varphi_{4} \sigma_{x}^{\prime}\right)}{\partial x}\right) \varphi_{2} \quad \text { o } \quad\left(\frac{\partial\left(\varphi_{4} \sigma_{x}^{\prime}\right)}{\partial x}\right)\left(\varphi_{2} \boldsymbol{u}^{\prime}\right) \quad \text { 0 }
\end{aligned}
$$

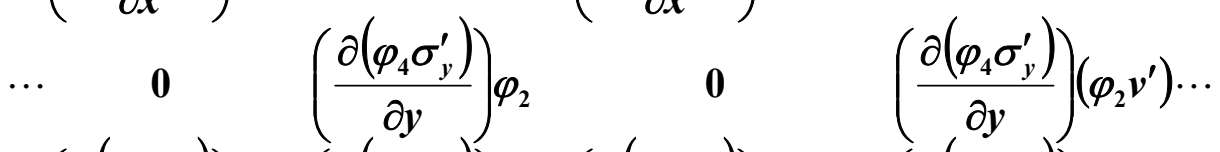

$$
\begin{aligned}
& \left(\frac{\partial\left(\varphi_{4} \tau_{x y}^{\prime}\right)}{\partial y}\right) \varphi_{2}\left(\frac{\partial\left(\varphi_{4} \tau_{x y}^{\prime}\right)}{\partial x}\right) \varphi_{2}\left(\frac{\partial\left(\varphi_{4} \tau_{x y}^{\prime \prime}\right)}{\partial y}\right)\left(\varphi_{2} u^{\prime}\right)\left(\frac{\partial\left(\varphi_{4} \tau_{x y}^{\prime}\right)}{\partial x}\right)\left(\varphi_{2} v^{\prime}\right) \\
& \left(\frac{\partial\left(\varphi_{4} \sigma_{x}^{\prime}\right)}{\partial x}\right) \varphi_{3} \quad \text { o } \quad\left(\frac{\partial\left(\varphi_{4} \sigma_{x}^{\prime}\right)}{\partial x}\right)\left(\varphi_{3} \boldsymbol{u}^{\prime}\right) \quad \text { 0 }
\end{aligned}
$$

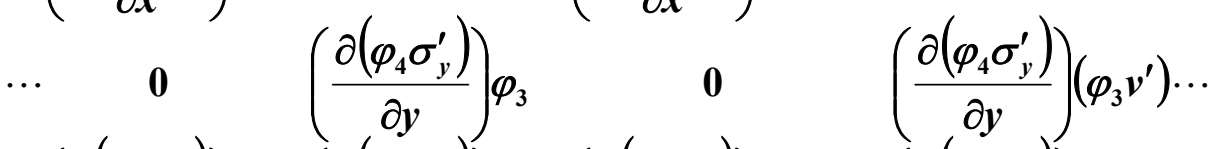

$$
\begin{aligned}
& \left(\frac{\partial\left(\varphi_{4} \tau_{x y}^{\prime}\right)}{\partial y}\right) \varphi_{3}\left(\frac{\partial\left(\varphi_{4} \tau_{x y}^{\prime}\right)}{\partial x}\right) \varphi_{3} \quad\left(\frac{\partial\left(\varphi_{4} \tau_{x y}^{\prime}\right)}{\partial y}\right)\left(\varphi_{3} u^{\prime}\right)\left(\frac{\partial\left(\varphi_{4} \tau_{x y}^{\prime}\right)}{\partial x}\right)\left(\varphi_{3} v^{\prime}\right) \\
& \left.\begin{array}{cccc}
\left(\frac{\partial\left(\varphi_{4} \sigma_{x}^{\prime}\right)}{\partial x}\right) \varphi_{4} & \mathbf{0} & \left(\frac{\partial\left(\varphi_{4} \sigma_{x}^{\prime}\right)}{\partial x}\right)\left(\varphi_{4} u^{\prime}\right) & \mathbf{0} \\
0 & \left(\frac{\partial\left(\varphi_{4} \sigma_{y}^{\prime}\right)}{\partial y}\right) \varphi_{4} & 0 & \left.\frac{\partial\left(\varphi_{4} \sigma_{y}^{\prime}\right)}{\partial y}\right)\left(\varphi_{4} v^{\prime}\right) \\
\left(\frac{\partial\left(\varphi_{4} \tau_{x y}^{\prime}\right)}{\partial y}\right) \varphi_{4} & \left(\frac{\partial\left(\varphi_{4} \tau_{x y}^{\prime}\right)}{\partial x}\right) \varphi_{4} & \left(\frac{\partial\left(\varphi_{4} \tau_{x y}^{\prime}\right)}{\partial y}\right)\left(\varphi_{4} u^{\prime}\right) & \left(\frac{\partial\left(\varphi_{4} \tau_{x y}^{\prime}\right)}{\partial x}\right)\left(\varphi_{4} v^{\prime}\right)
\end{array}\right]
\end{aligned}
$$


Caso nós do contorno do problema sejam enriquecidos, a matriz $\boldsymbol{A}_{\Gamma_{i}}$ dada pela eq.(4.13) terá uma dimensão de (24 x 4), como mostra a eq.(E.9).

Linhas 1, 2 e 3 da matriz $\boldsymbol{A}_{\Gamma_{i}}$ :

$\left[\begin{array}{cccc}\left(n_{x} \varphi_{1}\right) \psi_{1} & 0 & \left(n_{x} \varphi_{1}\right) \psi_{2} & 0 \\ 0 & \left(n_{y} \varphi_{1}\right) \psi_{1} & 0 & \left(n_{y} \varphi_{1}\right) \psi_{2} \\ \left(n_{y} \varphi_{1}\right) \psi_{1} & \left(n_{x} \varphi_{1}\right) \psi_{1} & \left(n_{y} \varphi_{1}\right) \psi_{2} & \left(n_{x} \varphi_{1}\right) \psi_{2}\end{array}\right]$

Linhas 4, 5 e 6 da matriz $\boldsymbol{A}_{\Gamma_{i}}$ :

$\left[\begin{array}{cccc}\boldsymbol{n}_{x}\left(\varphi_{1} \sigma_{x}^{\prime}\right) \psi_{1} & \mathbf{0} & \boldsymbol{n}_{x}\left(\varphi_{1} \sigma_{x}^{\prime}\right) \psi_{2} & \mathbf{0} \\ \mathbf{0} & \boldsymbol{n}_{y}\left(\varphi_{1} \sigma_{y}^{\prime}\right) \psi_{1} & \mathbf{0} & \boldsymbol{n}_{y}\left(\varphi_{1} \sigma_{y}^{\prime}\right) \psi_{2} \\ \boldsymbol{n}_{y}\left(\varphi_{1} \tau_{x y}^{\prime}\right) \psi_{1} & \boldsymbol{n}_{x}\left(\varphi_{1} \tau_{x y}^{\prime}\right) \psi_{1} & \boldsymbol{n}_{y}\left(\varphi_{1} \tau_{x y}^{\prime}\right) \psi_{2} & \boldsymbol{n}_{x}\left(\varphi_{1} \tau_{x y}^{\prime}\right) \psi_{2}\end{array}\right]$

Linhas 7, 8 e 9 da matriz $\boldsymbol{A}_{\Gamma_{i}}$ :

$\left[\begin{array}{cccc}\left(n_{x} \varphi_{2}\right) \psi_{1} & 0 & \left(n_{x} \varphi_{2}\right) \psi_{2} & 0 \\ 0 & \left(n_{y} \varphi_{2}\right) \psi_{1} & 0 & \left(n_{y} \varphi_{2}\right) \psi_{2} \\ \left(n_{y} \varphi_{2}\right) \psi_{1} & \left(n_{x} \varphi_{2}\right) \psi_{1} & \left(n_{y} \varphi_{2}\right) \psi_{2} & \left(n_{x} \varphi_{2}\right) \psi_{2}\end{array}\right]$

Linhas 10,11 e 12 da matriz $\boldsymbol{A}_{\Gamma_{i}}$ :

$$
\left[\begin{array}{cccc}
\boldsymbol{n}_{x}\left(\varphi_{2} \sigma_{x}^{\prime}\right) \psi_{1} & \mathbf{0} & \boldsymbol{n}_{x}\left(\varphi_{2} \sigma_{x}^{\prime}\right) \psi_{2} & \mathbf{0} \\
\mathbf{0} & \boldsymbol{n}_{y}\left(\varphi_{2} \sigma_{y}^{\prime}\right) \psi_{1} & \mathbf{0} & \boldsymbol{n}_{y}\left(\varphi_{2} \sigma_{y}^{\prime}\right) \psi_{2} \\
\boldsymbol{n}_{y}\left(\varphi_{2} \tau_{x y}^{\prime}\right) \psi_{1} & \boldsymbol{n}_{x}\left(\varphi_{2} \tau_{x y}^{\prime}\right) \psi_{1} & \boldsymbol{n}_{y}\left(\varphi_{2} \tau_{x y}^{\prime}\right) \psi_{2} & \boldsymbol{n}_{x}\left(\varphi_{2} \tau_{x y}^{\prime}\right) \psi_{2}
\end{array}\right]
$$


Linhas 13,14 e 15 da matriz $\boldsymbol{A}_{\Gamma_{i}}$ :

$$
\left[\begin{array}{cccc}
\left(n_{x} \varphi_{3}\right) \psi_{1} & 0 & \left(n_{x} \varphi_{3}\right) \psi_{2} & 0 \\
0 & \left(n_{y} \varphi_{3}\right) \psi_{1} & 0 & \left(n_{y} \varphi_{3}\right) \psi_{2} \\
\left(n_{y} \varphi_{3}\right) \psi_{1} & \left(n_{x} \varphi_{3}\right) \psi_{1} & \left(n_{y} \varphi_{3}\right) \psi_{2} & \left(n_{x} \varphi_{3}\right) \psi_{2}
\end{array}\right]
$$

Linhas 16,17 e 18 da matriz $\boldsymbol{A}_{\Gamma_{i}}$ :

$$
\left[\begin{array}{cccc}
\boldsymbol{n}_{x}\left(\varphi_{3} \sigma_{x}^{\prime}\right) \psi_{1} & \mathbf{0} & \boldsymbol{n}_{x}\left(\varphi_{3} \sigma_{x}^{\prime}\right) \psi_{2} & \mathbf{0} \\
\mathbf{0} & \boldsymbol{n}_{y}\left(\varphi_{3} \sigma_{y}^{\prime}\right) \psi_{1} & \mathbf{0} & \boldsymbol{n}_{y}\left(\varphi_{3} \sigma_{y}^{\prime}\right) \psi_{2} \\
\boldsymbol{n}_{y}\left(\varphi_{3} \tau_{x y}^{\prime}\right) \psi_{1} & \boldsymbol{n}_{x}\left(\varphi_{3} \tau_{x y}^{\prime}\right) \psi_{1} & \boldsymbol{n}_{y}\left(\varphi_{3} \tau_{x y}^{\prime}\right) \psi_{2} & \boldsymbol{n}_{x}\left(\varphi_{3} \tau_{x y}^{\prime}\right) \psi_{2}
\end{array}\right]
$$

Linhas 19, 20 e 21 da matriz $\boldsymbol{A}_{\Gamma_{i}}$ :

$$
\left[\begin{array}{cccc}
\left(n_{x} \varphi_{3}\right) \psi_{1} & 0 & \left(n_{x} \varphi_{3}\right) \psi_{2} & 0 \\
0 & \left(n_{y} \varphi_{3}\right) \psi_{1} & 0 & \left(n_{y} \varphi_{3}\right) \psi_{2} \\
\left(n_{y} \varphi_{3}\right) \psi_{1} & \left(n_{x} \varphi_{3}\right) \psi_{1} & \left(n_{y} \varphi_{3}\right) \psi_{2} & \left(n_{x} \varphi_{3}\right) \psi_{2}
\end{array}\right]
$$

Linhas 22,23 e 24 da matriz $\boldsymbol{A}_{\Gamma_{i}}$ :

$$
\left[\begin{array}{cccc}
\boldsymbol{n}_{x}\left(\varphi_{4} \sigma_{x}^{\prime}\right) \psi_{1} & \mathbf{0} & \boldsymbol{n}_{x}\left(\varphi_{4} \sigma_{x}^{\prime}\right) \psi_{2} & \mathbf{0} \\
\mathbf{0} & \boldsymbol{n}_{y}\left(\varphi_{4} \sigma_{y}^{\prime}\right) \psi_{1} & \mathbf{0} & \boldsymbol{n}_{y}\left(\varphi_{4} \sigma_{y}^{\prime}\right) \psi_{2} \\
\boldsymbol{n}_{y}\left(\varphi_{4} \tau_{x y}^{\prime}\right) \psi_{1} & \boldsymbol{n}_{x}\left(\varphi_{4} \tau_{x y}^{\prime}\right) \psi_{1} & \boldsymbol{n}_{y}\left(\varphi_{4} \tau_{x y}^{\prime}\right) \psi_{2} & \boldsymbol{n}_{x}\left(\varphi_{4} \tau_{x y}^{\prime}\right) \psi_{2}
\end{array}\right]
$$

A matriz $\boldsymbol{Q}_{\Gamma_{i}}$ dada pela eq.(4.14) terá uma dimensão de (4 x 1), ou seja, sua dimensão não será alterada com esse tipo de enriquecimento. 